\title{
Dry Reforming of Methane Using Microwave Irradiated Metal Oxide/Coal Char Catalysts
}

Anthony Carter

West Virginia University, aecarter@mix.wvu.edu

Follow this and additional works at: https://researchrepository.wvu.edu/etd

Part of the Catalysis and Reaction Engineering Commons, Manufacturing Commons, Materials Science and Engineering Commons, and the Other Engineering Science and Materials Commons

\section{Recommended Citation}

Carter, Anthony, "Dry Reforming of Methane Using Microwave Irradiated Metal Oxide/Coal Char Catalysts" (2021). Graduate Theses, Dissertations, and Problem Reports. 10228.

https://researchrepository.wvu.edu/etd/10228

This Thesis is protected by copyright and/or related rights. It has been brought to you by the The Research Repository @ WVU with permission from the rights-holder(s). You are free to use this Thesis in any way that is permitted by the copyright and related rights legislation that applies to your use. For other uses you must obtain permission from the rights-holder(s) directly, unless additional rights are indicated by a Creative Commons license in the record and/ or on the work itself. This Thesis has been accepted for inclusion in WVU Graduate Theses, Dissertations, and Problem Reports collection by an authorized administrator of The Research Repository @ WVU. For more information, please contact researchrepository@mail.wvu.edu. 


\title{
Dry Reforming of Methane Using Microwave Irradiated Metal Oxide/Coal Char Catalysts \\ Anthony Carter
}

\author{
Thesis submitted to the \\ Benjamin M. Statler College of Engineering and Mineral Resources \\ at West Virginia University \\ In Partial Fulfillment of the Requirements for the Degree \\ MASTER OF SCIENCE IN MATERIAL SCIENCE AND ENGINEERING
}

Edward Sabolsky, Ph.D., Chair

Terence Musho, Ph.D.

Charter Stinespring, Ph.D.

Konstantinos Sierros, Ph.D.

Department of Mechanical and Aerospace Engineering

Morgantown, West Virginia

August 2021

Keywords: Microwave, catalyst, coal, char, molybdate, dry reforming, alkaline, metal, oxides, syngas, reaction, coupling 


\begin{abstract}
Dry Reforming of Methane Using Microwave Irradiated Metal Oxide/Coal Char Catalysts
\end{abstract}

\begin{abstract}
Anthony Carter
This research focuses on the synthesis of both shaped and amorphous powder materials, the combination of these materials with dried Powder River Basin (PRB) coal char, and their reactionary properties with methane and carbon dioxide gasses with conventional and microwave (MW) heating. The first goal of this project was to synthesize shaped micro and nano sized particles with ideal dielectric properties for converting electromagnetic energy into heat and proven capabilities of activating methane. These particles were synthesized via solvothermal, hydrothermal, and co-preceptory treatments alone and onto the surface of dried PRB coal char. PRB is a sub-bituminous, low-ranking coal (LRC) containing impurities processing ideal properties for heating using microwave radiation. MW irradiation of metal oxides and char improved conversion of reforming gasses $\left(\mathrm{CH}_{4}\right.$ and $\left.\mathrm{CO}_{2}\right)$, reacting at lower temperatures than typically used with conventional heating methods. Lower DRM temperature parameters translate into diminished energy requirements, optimizing cost minimalization for a manufacturer. Outlet stream gases were monitored to examine effects of altering a particle's morphology, size, and shape, and the products that evolve from these reactor experiments. Additionally, varying the wt $\%$ of the catalysts on the dried PRB coal char were tested. Characterizations were completed using scanning electron microscopy (SEM), X-ray diffraction (XRD), temperature-controlled desorption (TPD), thermogravimetric analysis (TGA), gas chromatography (GC), and transmission line and free space method dielectric testing on all the materials synthesized. The purpose of this research was to improve understandings and the development of low-rank coal char to fuel synthesis, evaluate solid/gas interactions between Mo-based metal oxides, PRB coal char, and reforming gases, analyze the effects of MW irradiation use in these reactions compared to conventional heating methods, observe the effects of metal oxide particle sizes/morphologies and their effects on reforming reactions, and increase future viability and applicability of these processes.
\end{abstract}




\section{Table of Contents}

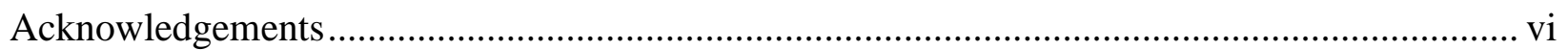

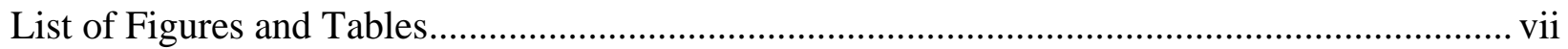

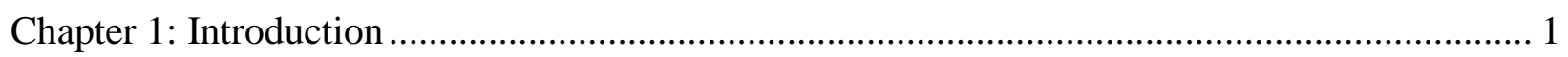

1.1: Brief History of Coal in the United States ..................................................................... 1

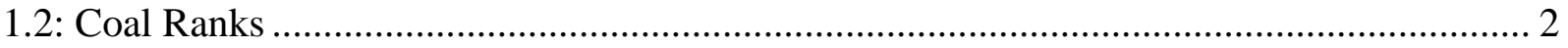

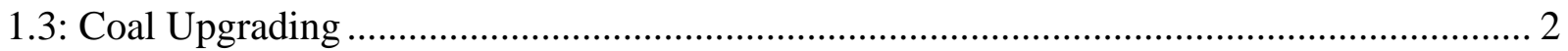

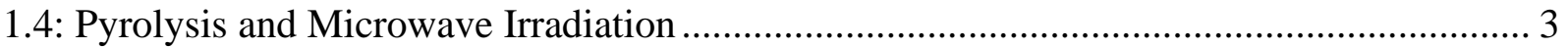

1.5: Combining Principles: DRM, Hydropyrolysis, and Microwave Induction ......................... 5

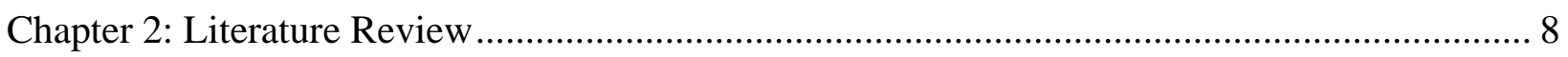

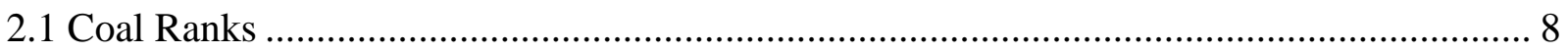

2.2 Coal Upgrading into Value-Added Chemicals................................................................. 9

2.2.1 Integrated Gasification-Combined Cycle (IGCC) ...................................................... 11

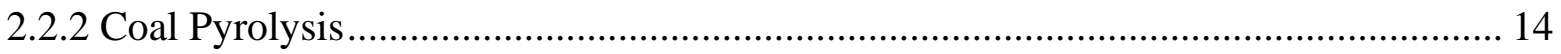

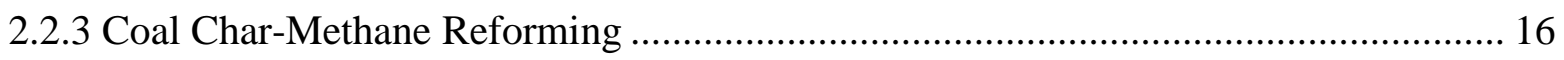

2.2.4 Pyrolysis Optimizations and Improvements ................................................................ 18

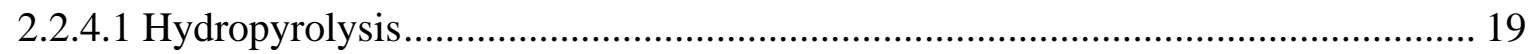

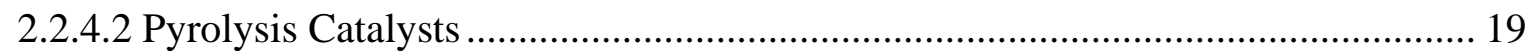

2.2.4.3 Microwave Pyrolysis of LRC ……………………....................................... 20

2.2.5 Hydropyrolysis and Methane Cracking Catalysts ..................................................... 22

2.2.5.1 Methane Upgrading - Catalytic Interactions....................................................... 22

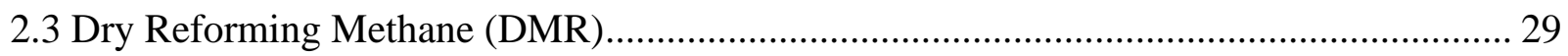

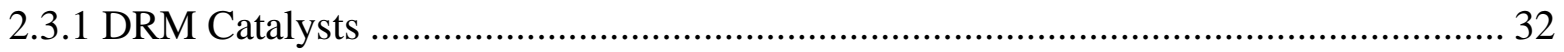

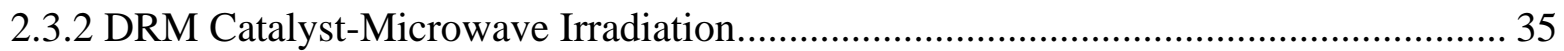

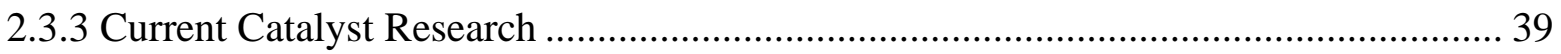

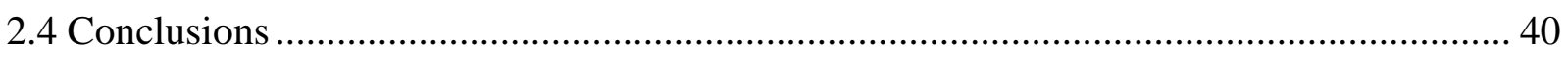

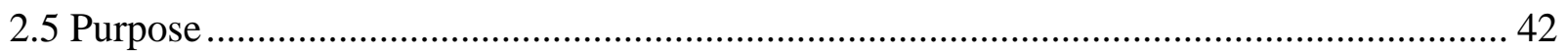

Chapter 3: Synthesis and Dielectric Properties of Shaped Metal Oxides Suitable for the MW-

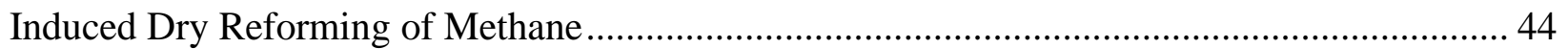

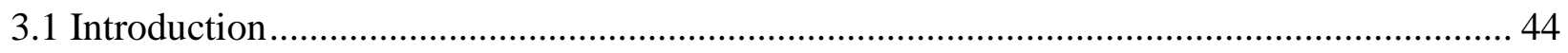


3.2 Experimental Procedures: Shaped Catalyst Synthesis ...................................................... 51

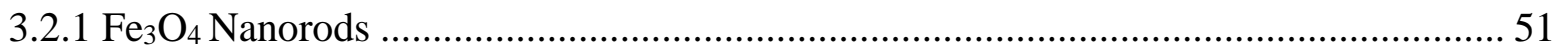

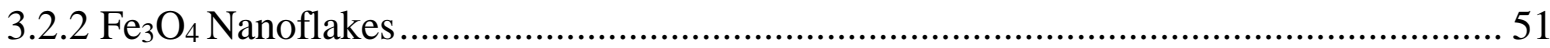

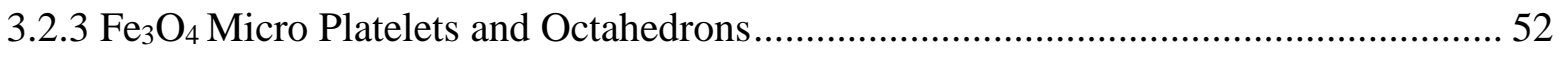

3.2.3.1 Reduction of $\alpha-\mathrm{Fe}_{2} \mathrm{O}_{3}$ to $\mathrm{Fe}_{3} \mathrm{O}_{4}$ platelets and octahedral particles........................ 53

3.2.4 $\mathrm{CaMoO}_{4}$ Micro-Needles/Platelet Clusters ………………........................................ 53

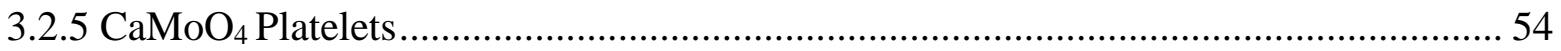

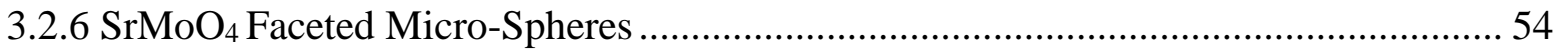

3.2.7 Particle Characterizations ……………………...................................................... 55

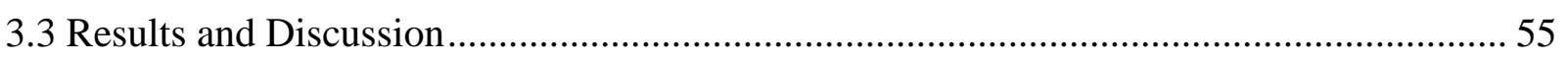

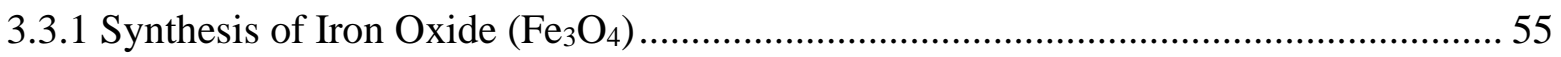

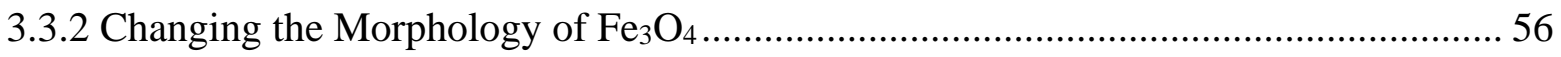

3.3.3 Effects of Changing Synthesis Conditions of $\mathrm{Fe}_{3} \mathrm{O}_{4}$ Particles...................................... 58

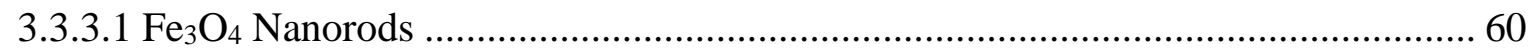

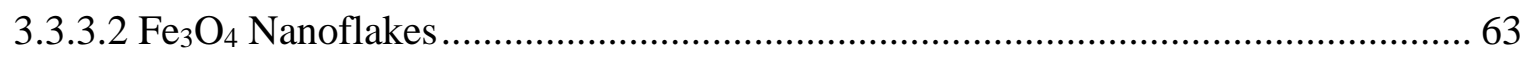

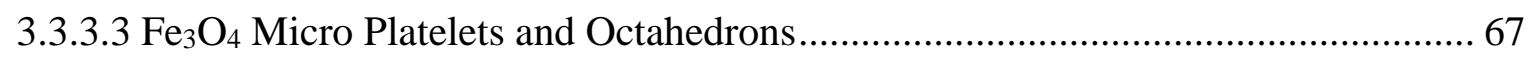

3.3.4 Synthesis of Calcium and Strontium Molybdate Particles and Morphological Changes .

3.3.4.1 $\mathrm{CaMoO}_{4}$ Needles, Doughnuts and Platelets..................................................... 73

3.3.4.2 $\mathrm{SrMoO}_{4}$ Multifaceted Micro Spheres …………….......................................... 78

3.3.5 Dielectric Comparisons of Shaped vs. Stock Metal Oxides ......................................... 80

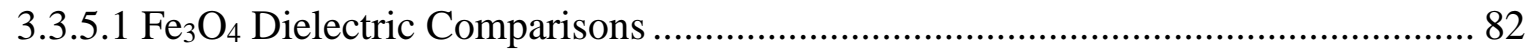

3.3.5.2 $\mathrm{CaMoO}_{4}$ and $\mathrm{SrMoO}_{4}$ Dielectric Comparisons …………………………......... 83

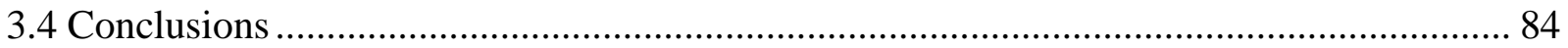

Chapter 4: Microwave Irradiation of Decorated Dried PRB Coal Char Catalysts in Dry

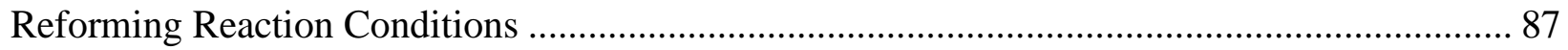

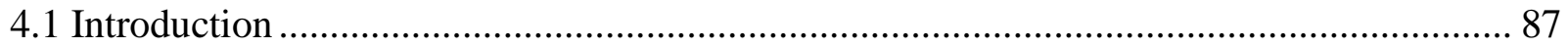

4.2 Experimental Procedures: $\mathrm{Me}_{\mathrm{x}} \mathrm{O}_{\mathrm{y}} /$ Char Catalyst Preparation \& Testing ........................... 95

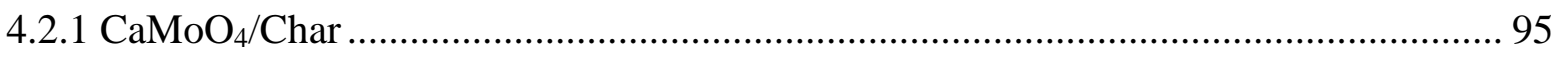

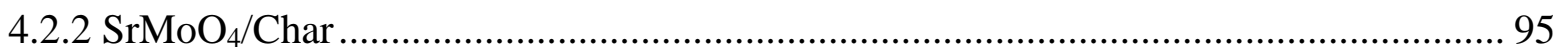

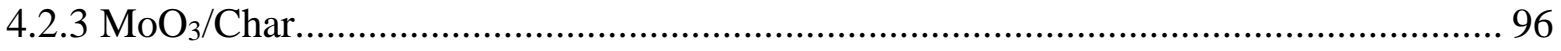

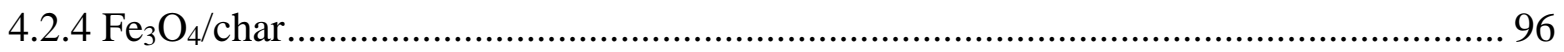


4.2.5 Shaped and Stock $\mathrm{M}_{\mathrm{x}} \mathrm{O}_{\mathrm{y}}$ Particles Both Mechanically Mixed with PRB Coal Char and

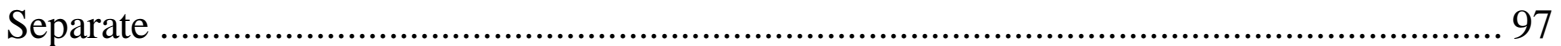

4.2.6 Microwave Reactor and Gas Chromatography Analysis ............................................ 98

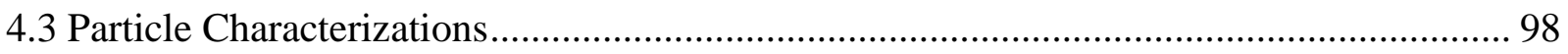

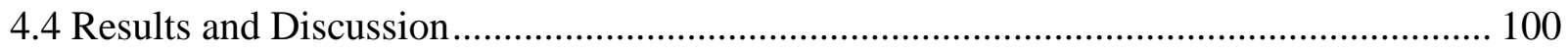

4.4.1 MWDRM Processing Shaped $\mathrm{Me}_{\mathrm{x}} \mathrm{O}_{\mathrm{y}}$ Catalysts vs. Stock Materials .......................... 101

4.4.2 MWDRM Processing with PRB Coal Char ............................................................ 113

4.4.3 MWDRM Processing with $\mathrm{Fe}_{3} \mathrm{O}_{4}$ Mechanically Mixed and Impregnated on PRB Coal

Char 123

4.4.4 MWDRM Processing with $\mathrm{SrMoO}_{4}$ Mechanically Mixed and Impregnated on PRB

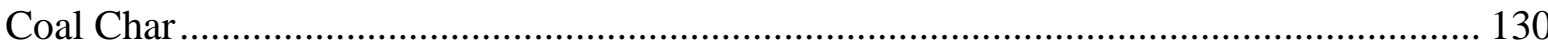

4.4.5 MWDRM Processing with $\mathrm{CaMoO}_{4}$ and $\mathrm{MoO}_{3}$ Impregnated on PRB Coal Char .... 138

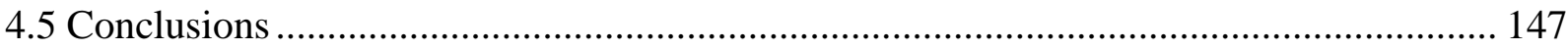

4.5.1 Microwave Irradiated Shaped $\mathrm{Me}_{\mathrm{x}} \mathrm{O}_{\mathrm{y}}$ Catalysts for DRM Processing ...................... 148

4.5.2 MWDRM Processing of $\mathrm{Me}_{\mathrm{x}} \mathrm{O}_{\mathrm{y}}$ Catalysts with Dried PRB Coal Char ..................... 150

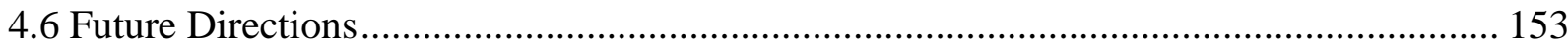

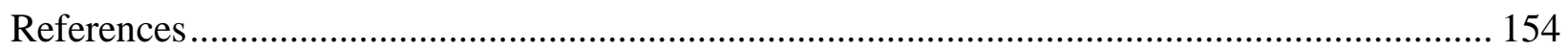




\section{Acknowledgements}

I would like to thank my advisor, Dr. Edward Sabolsky, for his guidance, patience, and advice throughout my graduate studies and research. His direction helped me reflect upon my own thinking and make better decisions when researching. Next, I would like to thank Dr. Christina Wildfire and Dr. Daniel Haynes for providing advice and assisting with many of the material characterizations needed for my research. I would also like to thank Dr. John $\mathrm{Hu}$ and his research team for their assistance in the laboratory. I would like to thank NETL for providing the funding for this research with grant number FE-FE0026825. I would like to thank my colleagues in 200 for all there help and support. At the beginning of my graduate research, they taught me how to operate the equipment needed for my research and guide me into becoming a better researcher overall. They provided advice and kept me on track when I needed it most. Finally, I would like to thank my family for their unending support and always believing in me. Without your support I would not have made it to where I am today. Thank you. 


\section{List of Figures and Tables}

Figure 2.1: Coal fixed carbon percentage vs. gross caloric value [8]

Figure 2.2: Block diagram of the DCL process (NETL.doe.gov) [10]

Figure 2.3: Simplified set of major gasification reactions (Wang, T., 2017) [12]

Figure 2.4: Simplified diagram of coal gasification (Wang, T., 2017) [14]

Figure 2.5: Effects of temperature, pressure and heating rate on char and tar yields from the pyrolysis of Loy Yang lignite (Sathe, Chirag, et al.). [15]

Figure 2.6: Methane cracking over a bed of various mixtures of raw char and quarts at $1173 \mathrm{~K}$ (Sun, Zhi-Qiang, et al.). [17]

Figure 2.7: Methane conversions at various temperatures over bituminous coal char (Sun, ZhiQiang, et al.). [17]

Figure 2.8: The Superoxide radical $\mathrm{O}^{2-}$ of a metal oxide adsorbing a proton of $\mathrm{CH}_{4}$ in close vicinity (Schwach, Pan, \& Bao, 2017). [23]

Figure 2.9: Illustration of reforming gasses adsorbing and reacting with metal oxide basic sites (Aziz, Jalil, Wongsakulphasatch, \& Vo, 2020Figure 2.10: Mo/HZSM-5 zeolite catalysts and the reaction mechanisms of methane dehydrogenation and coupling to ethylene (Zhou et al., 2012). [24]

Figure 2.10: Mo/HZSM-5 zeolite catalysts and the reaction mechanisms of methane dehydrogenation and coupling to ethylene (Zhou et al., 2012). [26]

Figure 2.11: Effect of gas flow rate on production yield under $\mathrm{N} 2, \mathrm{PG}$, and $\mathrm{PG}$ over $\mathrm{Ni} / \mathrm{MgO}$ on composition of $\operatorname{tar}$ (Zhu et al.). [28]

Figure 2.12: Methane adsorption and coadsorption of $\mathrm{CO}$ and $\mathrm{CH}_{4}$ on $\mathrm{MgO}$ edges and corners. (Schwach et al.). [29]

Figure 2.13: Comparison of the conversions of $\mathrm{CO}_{2}$ and $\mathrm{CH}_{4}$ obtained in the dry reforming experiments over the catalysts FY5 (experiment DR3) and $\mathrm{FY} 5+\mathrm{Ni}^{2} / \mathrm{Al}_{2} \mathrm{O}_{3}$ (experiment DR4) (B. Fidalgo et al., 2011). [38]

Figure 3.1: Pourbaix Diagram for Fe [56]

Figure 3.2: $\mathrm{SEM}$ image of preliminary $\mathrm{Fe}_{3} \mathrm{O}_{4}$ nanorod synthesis results from a quick injection of ethylenediamine. [58]

Figure 3.3: SEM imaging of initial nanorod synthesis in open atmosphere (top) vs. S3.1.1 (bottom). [60]

Figure 3.4: $\mathrm{XRD}$ patterns of $\mathrm{Fe}_{3} \mathrm{O}_{4}$ nanoparticles with altered ethylenediamine concentrations of $3 \mathrm{M}$ (S3.1.2), $4 \mathrm{M}$ (S3.1.1), and $5 \mathrm{M}$ (S3.1.3). [62] 
Figure 3.5: SEM images of morphological changes seen in $\mathrm{Fe}_{3} \mathrm{O}_{4}$ only by altering the concentration of sodium acetate: $322 \mathrm{mg}$ (S3.2.1), $270 \mathrm{mg}$ (S3.2.2), $238 \mathrm{mg}$ (S3.2.3), $222 \mathrm{mg}$ (S3.2.4), and $195 \mathrm{mg}(\mathrm{S} 3.2 .5)$. [64]

Figure 3.6: $\mathrm{SEM}$ imaging of $\mathrm{Fe}_{3} \mathrm{O}_{4}$ particle using $195 \mathrm{mg}$ of $\mathrm{NaAc}$ while stirring at $150 \mathrm{rpm}$ for 3 hours (S3.2.6). [65]

Figure 3.7: $\mathrm{SEM}$ imaging of $\mathrm{Fe}_{3} \mathrm{O}_{4}$ particle using $195 \mathrm{mg}$ of $\mathrm{NaAc}$ while stirring at $150 \mathrm{rpm}$ for 6 hours (S3.2.7). [66]

Figure 3.8: XRD pattern of $\mathrm{Fe}_{3} \mathrm{O}_{4}$ nanoparticles (S3.2.7) with a $\mathrm{NaAc}$ concentration of $195 \mathrm{mg}$, stirred at $150 \mathrm{rpm}$ for 6 hours. [66]

Figure 3.9: $\mathrm{XRD}$ pattern of initial synthesis attempt (S3.3.1) at micron sized $\mathrm{Fe}_{3} \mathrm{O}_{4}$ platelets showing traces of residual $\mathrm{Fe}_{2} \mathrm{O}_{3}$. [68]

Figure 3.10: SEM imaging of $\mathrm{Fe}_{2} \mathrm{O}_{3}(\mathrm{~A})$ and $\mathrm{Fe}_{3} \mathrm{O}_{4}$ (C) micro-platelet formations from literature vs. S3.3.1 of $\mathrm{Fe}_{2} \mathrm{O}_{3}$ (B) and $\mathrm{Fe}_{3} \mathrm{O}_{4}$ (D) micro-platelet formation (Zhao et al. 2018). [69]

Figure 3.11: XRD pattern of S3.3.2 (octahedrons) and S3.3.3 (platelets). [70]

Figure 3.12: $\mathrm{SEM}$ images of $\mathrm{Fe}_{2} \mathrm{O}_{3}$ (left) and $\mathrm{Fe}_{3} \mathrm{O}_{4}$ (right) of $\mathrm{S} 3.3 .2$ micro-octahedrons (A, B) and S3.3.3 micro platelet $(\mathrm{C}, \mathrm{D})$ formations. [71]

Figure 3.13: SEM images of $\mathrm{CaMoO}_{4}$ clusters and needles, S3.4.1 (left) and S3.4.1 repeated (right). [74]

Figure 3.14: SEM images of Sample $4(\mathrm{~S} 3.4 .4)\left(\mathrm{CaMoO}_{4}\right)$ with a needle dominant morphology. [75]

Figure 3.15: SEM images of $\mathrm{CaMoO}_{4}$ Sample 2 (S3.4.2) (top) and Sample 3 (S3.4.3) (middle) compared to Sample 5 (S3.4.5) (bottom). [76]

Figure 3.16: $\mathrm{SEM}$ images of (S3.4.6), $\mathrm{CaMoO}_{4}$ final platelet morphology. [77]

Figure 3.17: XRD pattern comparison of different morphological samples of $\mathrm{CaMoO}_{4}, \mathrm{~S} 3.4 .1$ through S3.4.6. [78]

Figure 3.18: SEM imaging of $\mathrm{SrMoO}_{4}$ multi-faceted spheres after hydrothermal processing. [79]

Figure 3.19: XRD pattern of $\mathrm{SrMoO}_{4}$ faceted spheres (S3.5.1) [80]

Figure 4.1: A schematic diagram of the reaction network of dry reforming methane (Bian, Das, Wai, Hongmanorom, \& Kawi, 2017). [88]

Figure 4.2: Experimental setup for variable-frequency MW reactor (Robinson et al., 2020) [99]

Figure 4.3: Volumetric percentages of outlet stream gasses over $\mathrm{SrMoO}_{4}$ catalyst within a DRM environment conventionally heated at $600{ }^{\circ} \mathrm{C}$. [103]

Figure 4.4: $\mathrm{Fe}_{3} \mathrm{O}_{4}$ samples $\mathrm{S} 3.1 .1$ and $\mathrm{S} 3.2 .7 \mathrm{CH}_{4}$ and $\mathrm{CO}_{2}$ conversion rates at $600{ }^{\circ} \mathrm{C}$ within a $5.85 \mathrm{GHz}$ MW reactor under dry reforming conditions [104] 
Figure 4.5: $\mathrm{Fe}_{3} \mathrm{O}_{4}$ samples $\mathrm{S} 3.1 .1$ outlet stream vol\% of $\mathrm{H}_{2}, \mathrm{CO}$, and $\mathrm{C}_{2} \mathrm{H}_{6}$ at $600{ }^{\circ} \mathrm{C}$ within a $5.85 \mathrm{GHz} \mathrm{MW}$ reactor under dry reforming conditions [105]

Figure 4.6: XRD patterns of $\mathrm{Fe}_{3} \mathrm{O}_{4}$ sample $\mathrm{S} 3.1 .1$ and $\mathrm{S} 3.2 .7$ both pre and post MWDRM reaction. [106]

Figure 4.7: $\mathrm{SEM}$ imaging of $\mathrm{Fe}_{3} \mathrm{O}_{4}$ samples $\mathrm{S} 3.1 .1$ nanorods $(\mathrm{A}, \mathrm{B})$ and $\mathrm{S} 3.2 .7$ nanoflakes $(\mathrm{C}$, D) both pre (left) and post (right) MWDRM processing. [107]

Figure 4.8: $\mathrm{SrMoO}_{4} / \mathrm{SiC}$ sample $\mathrm{S} 3.5 .1 \mathrm{CH}_{4}$ and $\mathrm{CO}_{2}$ conversion rates at $600{ }^{\circ} \mathrm{C}$ within a 5.85 GHz MW reactor under dry reforming conditions. [110]

Figure 4.9: Ethylene and ethane volumetric flowrates in the MW reactor outlet stream using $\mathrm{SrMoO}_{4} / \mathrm{SiC}(\mathrm{S} 3.5 .1)$ as a catalyst. [111]

Figure 4.10: $\mathrm{SEM}$ imaging of $\mathrm{SrMoO}_{4} / \mathrm{SiC}$ sample S3.5.1 pre (left) and post (right) MWDRM processing. [112]

Figure 4.11: Conversion rates of $\mathrm{CH}_{4}$ and $\mathrm{CO}_{2}$ over $\mathrm{PRB}$ coal char within a $5.85 \mathrm{GHz} \mathrm{MW}$ reactor at 600 and $700{ }^{\circ} \mathrm{C}$. [114]

Figure 4.12: $\mathrm{Vol} \%$ of $\mathrm{H}_{2}$ and $\mathrm{CO}$ over $\mathrm{PRB}$ coal char within a $5.85 \mathrm{GHz} \mathrm{MW}$ reactor $600{ }^{\circ} \mathrm{C}$ [115]

Figure 4.13: Vol\% of $\mathrm{C}_{2} \mathrm{H}_{6}$ and $\mathrm{C}_{2} \mathrm{H}_{4}$ over $\mathrm{PRB}$ coal char within a 5.85 $\mathrm{GHz} \mathrm{MW}$ reactor 600 ${ }^{\circ} \mathrm{C}[115]$

Figure 4.14: SEM imaging of PRB coal char pre (left) and post (right) MWDRM processing at $600{ }^{\circ} \mathrm{C} .[116]$

Figure 4.15: Conversion rates of $\mathrm{CO}_{2}$ with altered inlet gas streams over PRB coal char within a $5.85 \mathrm{GHz} \mathrm{MW}$ reactor at $600{ }^{\circ} \mathrm{C}$. [117]

Figure 4.16: $\mathrm{CO}_{2}$-TPD intensity vs. temperature plot for dried PRB coal char [118]

Figure 4.17: $\mathrm{CO}_{2}$-TPD intensity vs. temperature plot for dried PRB coal char impregnated with $5 \mathrm{wt} \%$ of $\mathrm{CaMoO}_{4}$. [119]

Figure 4.18: $\mathrm{CO}_{2}$-TPD intensity vs. temperature plot for dried PRB coal char impregnated with $5 \mathrm{wt} \%$ of $\mathrm{SrMoO}_{4}$. [120]

Figure 4.19: $\mathrm{CO}_{2}$-TPD intensity vs. temperature plot for dried PRB coal char impregnated with $5 \mathrm{wt} \%$ of $\mathrm{MoO}_{3}$. [121]

Figure 4.20: $\mathrm{CH}_{4}$ Conversion rates of $\mathrm{Fe}_{3} \mathrm{O}_{4} / \mathrm{char}$ samples and char within a $5.85 \mathrm{GHz} \mathrm{MW}$ reactor in DRM conditions at $600{ }^{\circ} \mathrm{C}$. [124]

Figure 4.21: $\mathrm{CO}_{2}$ Conversion rates of $\mathrm{Fe}_{3} \mathrm{O}_{4} /$ char samples and char within a $5.85 \mathrm{GHz} \mathrm{MW}$ reactor in DRM conditions at $600{ }^{\circ} \mathrm{C}$. [125]

Figure 4.22: Ethylene outlet gas vol\% over $\mathrm{Fe}_{3} \mathrm{O}_{4} /$ char samples and char within a $5.85 \mathrm{GHz} \mathrm{MW}$ reactor in DRM conditions at $600{ }^{\circ} \mathrm{C}$.[126] 
Figure 4.23: Ethane outlet gas vol\% over $\mathrm{Fe}_{3} \mathrm{O}_{4} /$ char samples and char within a $5.85 \mathrm{GHz} \mathrm{MW}$ reactor in DRM conditions at $600{ }^{\circ} \mathrm{C}$. [127]

Figure 4.24: Pre (left) and post (right) reaction SEM imaging of $\mathrm{Fe}_{3} \mathrm{O}_{4} /$ char samples $\mathrm{S} 4.1 .1$ (A, B), S4.1.2 (C, D), S4.1.3 (E, F), and S4.1.4 (G, H). [128]

Figure 4.25: Example of PRB coal char agglomeration post MWDRM processing from $\mathrm{Fe}_{3} \mathrm{O}_{4}$ sample S4.1.4. [130]

Figure 4.26: $\mathrm{CH}_{4}$ Conversion rates of $\mathrm{SrMoO}_{4} / \mathrm{Char}$ samples compared against bare PRB coal char and $\mathrm{SrMoO}_{4} / \mathrm{SiC}$ within a $5.85 \mathrm{GHz} \mathrm{MW}$ reactor. [132]

Figure 4.27: $\mathrm{CO}_{2}$ Conversion rates of $\mathrm{SrMoO}_{4} / \mathrm{Char}$ samples compared against coal char and $\mathrm{SrMoO}_{4} / \mathrm{SiC}$ within a $5.85 \mathrm{GHz} \mathrm{MW}$ reactor. [132]

Figure 4.28: Ethylene outlet gas vol\% over $\mathrm{SrMoO}_{4} /$ char samples and char within a $5.85 \mathrm{GHz}$ MW reactor in DRM conditions. [133]

Figure 4.29: Ethane outlet gas vol\% over $\mathrm{SrMoO}_{4} /$ char samples and char within a $5.85 \mathrm{GHz}$ MW reactor in DRM conditions. [134]

Figure 4.30: $\mathrm{SEM}$ imaging of pre (left) and post (right) reaction $\mathrm{SrMoO}_{4} /$ char samples $\mathrm{S} 4.2 .1$ (A, B), S4.2.2 (C, D), 4.2.3 (E, F), S4.2.4 (G, H), and S4.2.1 at $700{ }^{\circ} \mathrm{C}$ (I). [135]

Figure 4.31: Quartz sphere discovered in sample S4.2.1 post MWDRM processing at $700{ }^{\circ} \mathrm{C}$ (left) and a close up of some $\mathrm{SrMoO}_{4}$ remaining on the glass sphere surface (right). [137]

Figure 4.32: $\mathrm{SEM}$ imaging of $\mathrm{CaMoO}_{4}(\mathrm{~S} 4.3 .1$, left $)$ and $\mathrm{MoO}_{3}$ (S4.3.2, right) impregnated on PRB coal char. [139]

Figure 4.33: $\mathrm{CH}_{4}$ Conversion rates of $\mathrm{S} 4.3 .1$ and $\mathrm{S} 4.3 .2$ samples compared against bare PRB coal char within a $5.85 \mathrm{GHz} \mathrm{MW}$ reactor at 600 and $700{ }^{\circ} \mathrm{C}$. [140]

Figure 4.34: $\mathrm{CO}_{2}$ Conversion rates of $\mathrm{S} 4.3 .1$ and $\mathrm{S} 4.3 .2$ samples compared against bare PRB coal char within a $5.85 \mathrm{GHz} \mathrm{MW}$ reactor at 600 and $700{ }^{\circ} \mathrm{C}$. [141]

Figure 4.35: Ethylene outlet gas vol\% over S4.3.1, S4.3.2, S4.3.3, and coal char within a 5.85 $\mathrm{GHz} \mathrm{MW}$ reactor in DRM conditions at 600 and $700{ }^{\circ} \mathrm{C}$. [143]

Figure 4.36: Ethane outlet gas vol\% over S4.3.1, S4.3.2, S4.3.3, and coal char within a 5.85 $\mathrm{GHz}$ MW reactor in DRM conditions at 600 and $700{ }^{\circ} \mathrm{C}$. [144]

Table 2.1: The complete reactionary mechanism pathways for DRM (Nikko and Aman, 2011) [30]

Table 2.2: Activity of different metals for $\mathrm{CH}_{4}$ reforming with $\mathrm{CO}_{2}(\mathrm{Hou}$, Chen, Fang, Zheng, Yashima, 2006) [33]

Table 3.1: Dielectric Properties of Synthesized Vs. Stock Particles at volumetric percentages between $5-10 \%$ suspended in paraffin wax [82]

Table 4.1: Shaped and Stock Particles Both Mixed and Unmixed with PRB Coal char [97] 
Table 4.2: $5 \mathrm{wt} \% \mathrm{Fe}_{3} \mathrm{O}_{4} /$ char samples tested in a $5.85 \mathrm{GHz} \mathrm{MW}$ reactor operating at $600{ }^{\circ} \mathrm{C}$ under DRM processing conditions [123]

Table 4.3: $\mathrm{SrMoO}_{4} / \mathrm{Char}$ samples tested in a $5.85 \mathrm{GHz} \mathrm{MW}$ reactor operating at $600{ }^{\circ} \mathrm{C}$ under DRM processing conditions [131]

Table 4.4: $\mathrm{CaMoO}_{4} / \mathrm{Char}$ and $\mathrm{MoO}_{3} / \mathrm{Char}$ samples tested in a $5.85 \mathrm{GHz} \mathrm{MW}$ reactor operating at $600{ }^{\circ} \mathrm{C}$ and $700{ }^{\circ} \mathrm{C}$ under DRM processing conditions [138]

Table 5.1: Summary of All Materials Tested Within MWDRM Conditions and Average Conversion Rates of the Reforming Gasses [150] 


\section{Chapter 1: Introduction}

\section{1: Brief History of Coal in the United States}

Early utilization of coal mined in the United States was primarily burned domestically and commercially to heat households, businesses, and factories until electrification was introduced in the mid- $20^{\text {th }}$ century. Additionally, coal was vital for producing iron and steel in high temperature blast furnaces, powering locomotives and marine vessels in the transportation industry, and manufacturing various fuels and chemicals. Chemical industries have used coal to produce a variety of chemicals including dyes, ammonia, explosives, and to a lesser degree, pharmaceuticals. Electrification shifted the chemical industry's previous reliance from coal to oil for the future of chemical processing in the 1950's that began limiting the applicability of coal as a power source. The industrial sector's focus shifted to use coal for producing steam, electricity, iron, steel, and some chemicals. More recently, the primary U.S. coal products have been power generation, coke production, and exports. Since peaking in 2009, coal consumption has been on a continued decline as the emergence of shale gas consumption has increased in popularity and a societal shift of using cleaner, more sustainable resources pushes coal combustion energy into obscurity. Coal's diminishing utility in the industrial sector has led to an increased interest in the development of other profitable applications within the chemical industry for producing fuels for the country's increasing energy demands. The United States has the highest share of the world's provided coal reserves (22\%), with an estimated 253 billion short tons in recoverable reserves (EIA). Because of the abundance of the United States' coal reserves and the heightened demand for future sustainable energy, it can be suggested that cleaner coal-derived fuels should play an expanded role in meeting these needs for the foreseeable future (Singh, S., et al.). 


\section{2: Coal Ranks}

Coal is classified into two main classes: high rank coal (HRC) and low rank coal (LRC). Most HRCs are used for steam generation and steel production due to their high caloric values that produce large amounts of heat when combusted. Inversely, LRCs have lower caloric values and produce more pollution when burned, leaving much to be desired as society focuses on cleaner, sustainable fuel sources as the next generation of energy production. Moreover, the United States' minable coal reserves possess an incredible energy potential because they consist of $\sim 52 \%$ LRC. Utilization of this large supply of LRC has promoted the use of several coal upgrading technologies such as coal-to-fuel (CTF) and high temperature heating (carbonization) for upgrading this undervalued coal supply. These methods exploit the overlooked, untapped potential of LRCs, converting them into increasingly profitable, cleaner liquid and gaseous fuels.

\section{3: Coal Upgrading}

Coal upgrading is defined as the processing of run of mine (ROM) coal by removal of some of the materials present via physical separation, thermal treatment, and chemical processing. This dissertation will mainly concern CTF processes will be covered while certain coking/carbonization topics will also be highlighted. Chemical processing of coal products CTF processes such as indirect coal liquefaction (ICL), direct coal liquefaction (DCL), and coal gasification (CG) convert coal into highly marketable fuels. Each of these technologies pretreat coal by means of pyrolysis where the coal enters a hot combustion environment created by burning other gaseous or liquid fuels in the absence of oxygen. Coal reacts and separates as the temperature rises, initially desorbing water vapor, $\mathrm{CO}_{2}$, and $\mathrm{N}_{2}$ gas. Devolatilization of heavier liquid hydrocarbons (coal tars) leave the remaining solid components to repolymerize in the form of char (Ozer, Mustafa, et al.). Lower pyrolysis temperatures cause coal to begin forming these chars and heavy volatile 
species that are trapped in the condensed phase. Conversely, higher temperatures react heavier coal tars and char further decomposing them into lighter gases (mainly $\mathrm{H}_{2}$ and $\mathrm{CO}$ ) for synthesis gas refinement and coal tars respectively, leaving more aromatic char structures (Ranzi, E., et al.). The remaining chars and tars from pyrolysis vary highly depending on the sourced PRB as each have their own compositions of impurities. To summarize, lighter pseudo-component (- $\left.\mathrm{CH}_{2}-\right)$ hydrocarbons $\left(\mathrm{C}_{2}-\mathrm{C}_{5}\right)$ and monoaromatic components (mainly benzene, toluene, and xylene) can evolve from pyrolysis in the form of chars and (Ranzi, E., et al.). Several studies have shown increased yield of tars and char formation from varying pressure, temperature, rapid heating, and heating via MW induction (Abdelsayed, Victor, et al., Sathe, Chirag, et al.). Increasing the yield of tar and char would intensify the profitability of LRCs, helping satisfy the need for cleaner, sustainable energy in the United States, and would provide more utility of LRC's in the chemical industry to produce the later fuels.

\section{4: Pyrolysis and Microwave Irradiation}

Uncatalyzed conventional coal pyrolysis requires temperatures of up to $1400^{\circ} \mathrm{C}$ to fully utilize final tar and char contents. Temperatures lower than $1100^{\circ} \mathrm{C}$ do not fully volatize the trapped hydrocarbons remaining in the coal macrostructure, so less aromatic char structures do not evolve in these conditions. Typically, conventionally heated reactors use large amounts of electricity to be brought to these high temperatures resulting in high utility costs. The trapped tars in the coal interact with the char via crosslinking resulting in the release of coal tars coinciding with some desorbed gases. These tars and gasses are highly valuable once refined later in the gasification process where they will be turned into calorically dense fuels compared to LRC. Increasing the temperature theoretically would yield higher tar amounts, however conventionally heating any reactor has limitations, as the heat transfer rate of the particles decreases during the reaction and 
the temperature plateaus (Ranzi, E., et al.). Similarly, high pressure can further increase tar formation but at the detriment of higher operational and capital costs. Recent studies have experimented with MW radiation to supplement the higher temperature requirements for increased coal tar yield while minimizing capital and operational costs (Abdelsayed, Victor, et al.). Post pyrolysis, after the tars and gasses have been separated, the remaining solids exit in the form of char. Char is fine solid particles of unreacted carbon and siliceous ash with high porosity. The porosity is highest in untreated chars derived from lower ranking coals and can be used as an adsorbing agent (NETL.doe.gov). High porosity char has been tested to adsorb mercury and $\mathrm{NO}_{\mathrm{x}}$ gas emissions, used as a precursor for producing activated carbon, and recycled back into the gasification process. Raw coal char is not as valuable as the gases and tars that are separated and can be considered the least desirable product of the pyrolysis process. PRB coal char often contains varying levels of environmentally unsafe impurities that can be released from the microstructure into the atmosphere when burnt. These impurities are frequently the still trapped within the char even after the pyrolysis treatment. Better utilization of char may prove to be beneficial as the industry continues to strive for cleaner energy.

MW irradiation exploits many of PRB's disparaging properties such as high moisture and sulfur content as both easily absorb the radiation. Since these components are evenly distributed throughout the coal, the coal bed also heats evenly when irradiated. Even distribution is also found regarding the solid char byproduct formed, as many of these impurities are trapped within the microstructure. MW reforming establishes other advantages such as lower capital costs due to the decreasing expense of MW reactors, installation compatibility, scalability for various design configurations, shortened startup time, immediate heating and a lower power consumption when compared to conventional heating (Singh, S., et al.). 


\section{5: Combining Principles: DRM, Hydropyrolysis, and Microwave Induction}

The reforming of methane requires high temperatures for activation to create value added fuels such as syngas or ideally heavy hydrocarbons (depending on the catalysts used and the process) once $\mathrm{H}^{+}$ion is removed from the methane structure. Oxidation of the methane molecule results in $\mathrm{H}_{2}$ gas and solid carbon that ends up accumulating throughout the reaction environment.

Coking is the primary reason for catalyst deactivation with methane reactions by covering a catalyst's active sites. A common way of removing this coking is with the addition of $\mathrm{CO}_{2}$ where it reacts with carbon to form $\mathrm{CO}$, another valuable synthesis gas. Although this is quite effective, even at a ratio of 1:1 for $\mathrm{CH}_{4}$ : $\mathrm{CO}_{2}$, carbon deposits still eventually deactivate normal catalysts, sparking the need for affordable anti-coking catalysts in the industry. Noble metal catalysts produce reasonably high levels of methane conversion, but the true benefit is their incredibly high resistance to carbon deposition known as coking. Unfortunately, these are immensely expensive and are not economically viable for industrial scale use.

A similar process, hydropyrolysis, uses a proton donating gas to upgrade LRC into valuable volatiles. Methane is heated to produce $\mathrm{H}^{+}$that is absorbed to the surface of the PRB where it reacts with hydrogen-deficient molecules before volatizing. PRB coal char could also accept these hydrogen protons readily as it has a lack of available hydrogen atoms within its chemical structure. This surface reaction can be seen occurring at the surface of char particles, but the reaction tends to require high temperatures. The heat transfer on coal surfaces have also been shown to decrease as the reaction rate increases, eventually stifling the methane-char reaction. Pyrolysis research has shown that the addition of certain metal oxide particles improves the conversion PRB coal char and methane in economically favorable reaction environments. Metal oxides such as tungstate and molybdate can interact with methane at lower temperatures $\left(\sim 500^{\circ} \mathrm{C}\right)$ than traditionally heating the 
gas. These oxides have shown to weaken the $\mathrm{C}-\mathrm{H}$ bonds in methane and in some instances assist in the coupling of the newly formed methyl radicals into heavier hydrocarbons. Metal oxides also absorb MW radiation to evenly distribute heat throughout a reactor bed during the pyrolysis process, especially those with high dielectric properties such as $\mathrm{Fe}_{3} \mathrm{O}_{4}, \mathrm{NiO}$, etc. Heat distribution helps prevent hotspots from occurring within the reactor, promoting distributed reforming and/or coupling between the hydrogen donating gas and the PRB. Combining these principles of DRM, hydropyrolysis, and MW inducible catalysts could vastly improve the production of syngas and other value-added chemicals from low-cost precursors and affordable catalysts.

The impurities that remain in PRB coal char particles can absorb MW radiation in the form of heat like that of the original PRB coal char. MW radiation is theorized to assist in the coupling of fine coal char particles with a natural gas such as methane for producing value added chemicals. Past studies have also shown coal char to assist with methane cracking at $900{ }^{\circ} \mathrm{C}$ for short periods of time before quickly losing its catalytic capabilities (Z. Sun et al.). The high temperatures of this reaction limit cost efficiency of the process in its current state and would require a drastic reduction in activation energy to increase the viability of utilizing coal char catalyst for activating methane. Improving the longevity of a coal char catalyst would also prove beneficial, as the coal char's reaction rate with methane quickly decreases when exposed to high temperatures. Also, coating the outside of coal char with high basicity oxides would reduce erosion of coal char catalysts from reactions with $\mathrm{CO}_{2}$ and assist with adsorbing $\mathrm{CO}_{2}$ to prevent coking from the primary methane reaction on the surface of the metal oxides. These same oxides will have higher surface areas contacting the coal char comparatively to simply mechanically mixing the two together. These could offer tertiary active sites between the char, metal oxide, and the gaseous reagents. These tertiary sites could offer coupling points for the methane and carbon dioxide to form heavier 
compounds, such as ethylene, ethane, etc. Currently, little research has been conducted for utilizing coal char as a catalyst, a catalyst support, or used as a MW absorbing bulk heat distributing particle. Research on methane/char reactions are very limited as well. Further research is needed to expand the understanding of the gas-solid reaction pathways between PRB coal char and methane gas, reduce the activation energy needed to upgrade methane, and explore the effect of MW radiation on the reaction. Additionally, this paper will cover the role of affordable, heterogeneous, metal oxide particles in methane conversion, interactions with MWs, effects of catalyst morphology and sizing on methane conversion and MW absorption, and coating coal char with these catalytic particles for MW processing. This paper aims to bridge gaps within the literature, providing a better understanding of PRB coal char's solid-gas interaction with methane and MW radiation to produce value-added fuels for the future of coal-derived energy as well as increase the utilization of coal derivatives. 


\section{Chapter 2: Literature Review}

\subsection{Coal Ranks}

Coals are not created equal, as there is a large variety that vary greatly, each with a wide range of chemical and physical properties. Coal can be categorized into two major groups and four main subgroups. HRCs

(Anthracite and Bituminous) have a high carbon content, low moisture, and a high caloric value ( $>24$ $\mathrm{MJ} / \mathrm{kg}$ ) perfectly suited for fueling steel foundry ovens and power plant boiler. PRBs (Sub-bituminous and Lignite) have lower carbon contents, high amounts of ash, and high moisture which results in a low

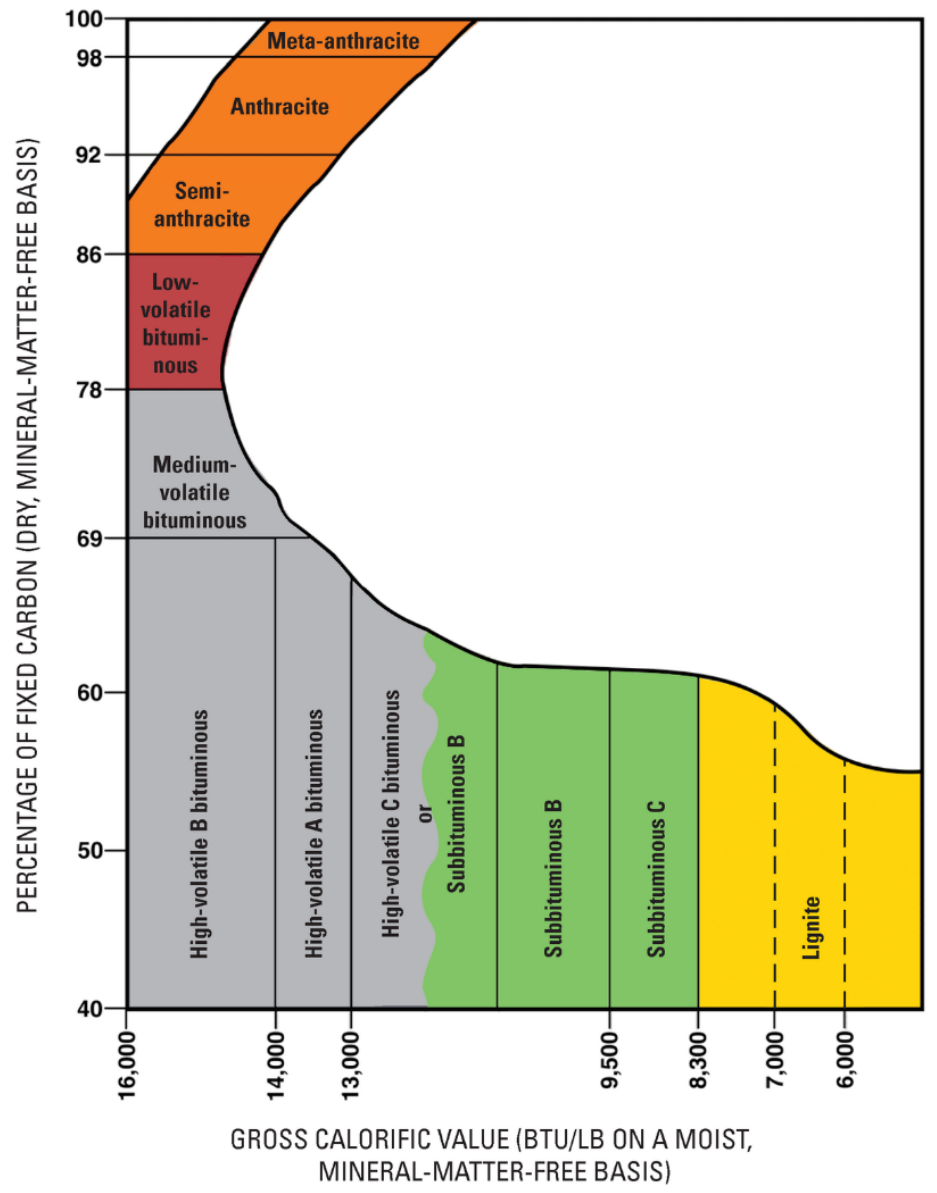

Figure 2.1: Coal fixed carbon percentage vs. gross caloric value

caloric value (<24 MJ/kg). Most LRC's have low sulfur contents, but some lignite coal has shown to have amounts as high as $10 \%$ in specific cases (Schobert, 2017). LRC's have a soft, dull, almost wood-like texture. These coal subgroups and their respective caloric values can be seen in Figure 2.1. LRCs are insufficient when burned compared to HRCs as the high moisture content absorbs heat that escapes the combustion process in the form of flue gas; this results in large heat losses. LRC's also emit higher levels of pollutants comparatively, further reducing 
their future applicability in the search for sustainable, cleaner energy sources. Despite these drawbacks, LRCs are still primarily used today for electric power generation worldwide, as they make up nearly half of the world's recoverable coal reserves.

\subsection{Coal Upgrading into Value-Added Chemicals}

While HRCs have mostly been used as the main source of fuel for coal-fired power plants in the United States, LRCs have been a topic of great interest as a feedstock for chemical industries. During the early to mid- $20^{\text {th }}$ century, coal technologies such as liquefaction, gasification, and carbonization began commercializing production to further utilize the stored potential of lowranking coal beyond that of combustion. These processes aim to convert coal into value-added products including syngas, activated carbon, coke, calorically dense fuels, and especially heavy hydrocarbons (coal tars) used to manufacture other value-added chemicals depending on the processing method. One of the first successful commercial attempts at converting coal into these value-added chemicals was done using direct coal liquefaction (DCL). Developed in the 1940's, DCL has been used to manufacture fuels by directly contacting coal with a catalyst in the presence of a solvent and add hydrogen as a proton donating gas to create a raw, liquid fuel precursor (NETL.doe.gov). The term "direct" is referring to the technique of bypassing the gasification process to form syngas and instead directly forms liquid fuels without further processing (NETL.doe.gov). DCL is considered a straightforward, efficient, two-step process when compared 
to indirect coal liquefaction (ICL) which is a three-step method. A simplified DCL process can be seen in Figure 2.2, highlighting the main parts of the process.

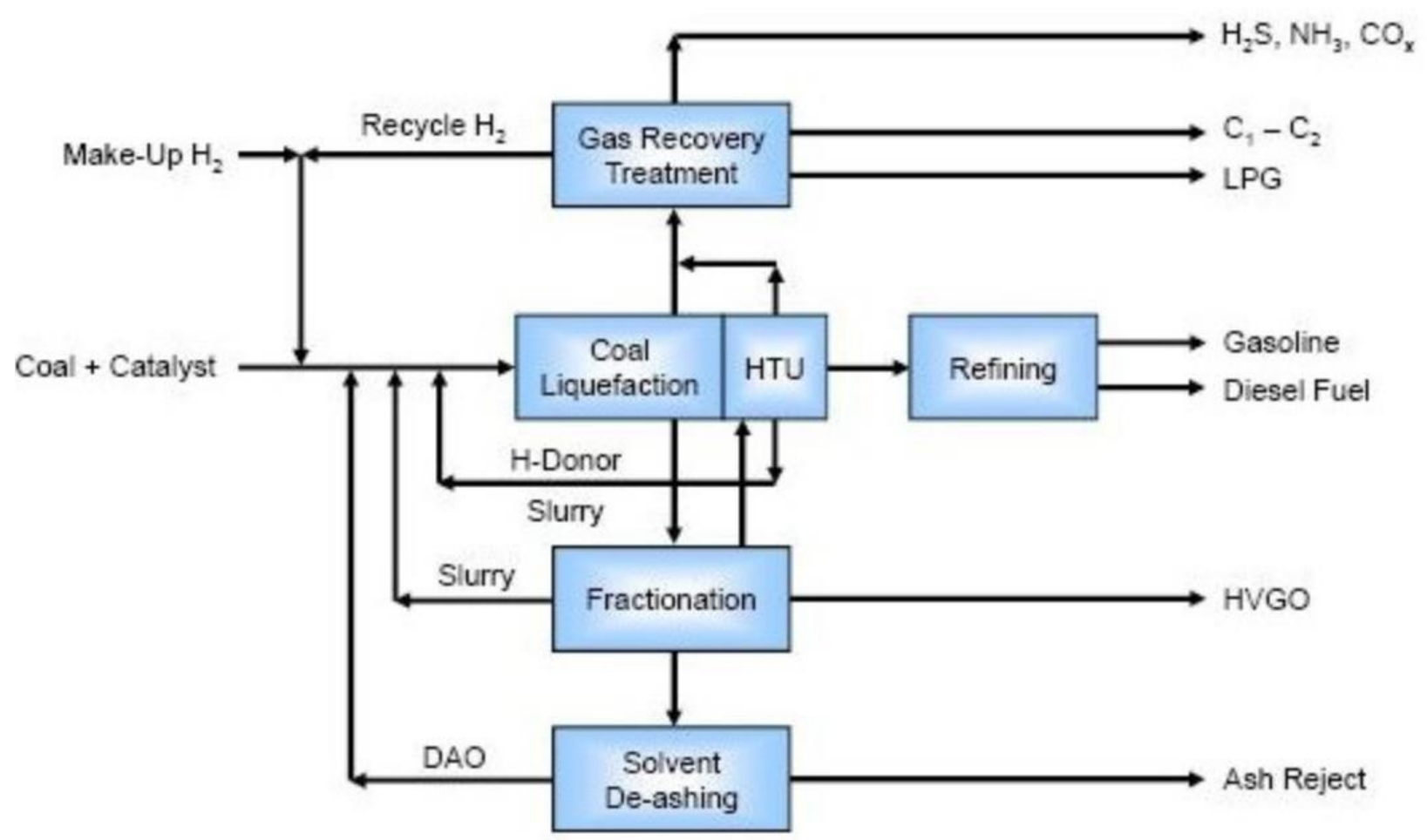

Figure 2.2: Block diagram of the DCL process (NETL.doe.gov)

DCL, however, does require an outside $\mathrm{H}_{2}$ source that would have to come from an additional gasified coal source or from the DCL reactor itself as seen above. The use of catalysts mixed with the coal and other innovations in reactor design paved the way for DCL to potentially be the next coal-based fuel of the future, but although effective, DCL harbors numerous downsides. DCL operates at mild temperatures and extremely high pressures to produce hydrocarbon fuels resulting in high capital intensity. It also consumes large amounts of water and releases large amounts of $\mathrm{CO}_{2}$ and various other pollutants into the atmosphere and water systems. Even with a relatively high coal-to-fuel efficiency of $65 \%$, DCL fell out of favor in the latter half of the 1980's to early 1990's. Many of these shortcomings were not considered economically or environmentally viable 
compared to cheap natural gas and oil, exacerbating the need for an alternative, sustainable fuel generation process as energy demands continued to grow.

Many of these processes fell to the wayside during the late 1940's as natural gas became more widely available and popular with its higher heating value and lack of contaminants. After the 1970's oil crisis, a resurgence of coal derived fuel research in liquefaction and gasification reemerged as a top priority for potentially supplementing future oil and natural gas fuel shortages. However, petroleum and gas prices eventually fell, and the incentive to construct new SNG facilities was eliminated as domestic resources were adequate to provide low-cost natural gas to the country. By the early 2000's, few commercial gasification systems remained leaving a handful in operation to this day. Currently, the emphasis on increased power generation and the better availability of high-performance fuel cells and turbines have provided a resurgence in highefficiency gasification systems to provide fuel. These new systems are focused on optimized highpurity synthesis gas for catalytic conversion to chemicals and power generating fuels for this new heightened market demand.

\subsubsection{Integrated Gasification-Combined Cycle (IGCC)}

Coinciding with liquefaction, gasification also reemerged as an oil/natural gas alternative by converting coal into hydrogen and carbon monoxide rich fuel gas. Gasification has continued to gather interest as it has evolved into what is known today as the Integrated Gasification Combined Cycle (IGCC). The purpose of IGCC is to produce electrical energy in a more environmentally friendly, efficient manner compared to the direct combustion of fuels. IGCC's cleaner approach is achieved by first gasifying solid/liquid fuels, cleaning the gases to remove harmful elements such as mercury, sulfur, and other trace element, and then burning the resulting cleaner synthesis gas (syngas) for power (Wang, T., 2017). IGCC processes start with gasification where the solid/liquid 
reactants are combusted to produce fuels and chemicals rather than heat. The goal of the gasification process is to conserve as much of original fuel's chemical energy as possible. Therefore, the feedstock is not fully combusted (oxidized). This partial oxidation of the feedstock fuel varies depending on the original fuel's origins. Light oxidants, such as $\mathrm{CO}_{2}$, may also be used to oxidize LRC and produce useful CO gas. These reactions can be extremely complicated; Figure 2.3 below provides a summarized set of major global reactions (Wang, T., 2017).

Heterogeneous reactions:

$\mathrm{C}(\mathrm{s})+1 / 2 \mathrm{O}_{2} \rightarrow \mathrm{CO} \quad \Delta \mathrm{H}_{\mathrm{R}}^{\circ}=-110.5 \mathrm{MJ} / \mathrm{kmol}$

$\mathrm{C}(\mathrm{s})+\mathrm{CO}_{2} \leftrightarrow 2 \mathrm{CO} \Delta \mathrm{H}_{\mathrm{R}}^{\circ}=+172.0 \mathrm{MJ} / \mathrm{kmol}$ (Gasification, Reverse Boudouard reaction) [R1.2]

$\mathrm{C}(\mathrm{s})+\mathrm{H}_{2} \mathrm{O}(\mathrm{g}) \rightarrow \mathrm{CO}+\mathrm{H}_{2} \quad \Delta \mathrm{H}^{\circ}{ }_{\mathrm{R}}=+131.4 \mathrm{MJ} / \mathrm{kmol}$ (Steam-Char Gasification) [R1.3]

$\mathrm{C}+2 \mathrm{H}_{2} \rightarrow \mathrm{CH}_{4}, \quad \Delta \mathrm{H}^{\circ}{ }_{\mathrm{R}}=-87.4 \mathrm{MJ} / \mathrm{kmol}$ (Hydrogasificaiton, Direct methanation) [R1.4]

Homogeneous reactions:

$$
\begin{array}{llll}
\mathrm{CO}+1 / 2 \mathrm{O}_{2} \rightarrow \mathrm{CO}_{2} & \Delta \mathrm{H}^{\circ}{ }_{\mathrm{R}}=-283.1 \mathrm{MJ} / \mathrm{kmol} & \text { [R1.5] } \\
\mathrm{CO}+\mathrm{H}_{2} \mathrm{O}(\mathrm{g}) \leftrightarrow \mathrm{CO}_{2}+\mathrm{H}_{2} & \Delta \mathrm{H}^{\circ}{ }_{\mathrm{R}}=-41.0 \mathrm{MJ} / \mathrm{kmol} & \text { (Water-gas shift) } \\
\mathrm{CO}+3 \mathrm{H}_{2} \leftrightarrow \mathrm{CH}_{4}+\mathrm{H}_{2} \mathrm{O} & \Delta \mathrm{H}^{\circ}{ }_{\mathrm{R}}=-205.7 \mathrm{MJ} / \mathrm{kmol} & \text { (Methanation) } \\
\mathrm{CH}_{\mathrm{m}} \mathrm{O}_{\mathrm{n}} \mathrm{N}_{\mathrm{o}} \mathrm{S}_{\mathrm{p}} \mathrm{Cl}_{\mathrm{q}} \rightarrow \mathrm{aCO}+\mathrm{bH}_{2}+\mathrm{cCH}_{4}+\mathrm{dC}_{2} \mathrm{H}_{2}+\mathrm{eN}_{2}+\mathrm{fHCl}+\mathrm{gH}_{2} \mathrm{~S}+\mathrm{hCOS} \text { (Volatile cracking) } & \text { [R1.8] } \\
\mathrm{CH}_{4}+1 / 2 \mathrm{O}_{2} \rightarrow \mathrm{CO}+2 \mathrm{H}_{2} & \Delta \mathrm{H}^{\circ}{ }_{\mathrm{R}}=-35.7 \mathrm{MJ} / \mathrm{kmol} \text { (Volatiles gasification via } \mathrm{CH}_{4} \text { ) } \\
\mathrm{C}_{2} \mathrm{H}_{2}+\mathrm{O}_{2} \rightarrow 2 \mathrm{CO}+\mathrm{H}_{2} & \Delta \mathrm{H}^{\circ}{ }_{\mathrm{R}}=-447.83 \mathrm{MJ} / \mathrm{kmol} \text { (Volatiles gasification via } \mathrm{C}_{2} \mathrm{H}_{2} \text { ) [R1.10] } \\
\mathrm{H}_{2}+1 / 2 \mathrm{O}_{2} \rightarrow \mathrm{H}_{2} \mathrm{O} & \Delta \mathrm{H}^{\circ}{ }_{\mathrm{R}}=-242 \mathrm{MJ} / \mathrm{kmol} & \text { [R1.11] }
\end{array}
$$

Figure 2.3: Simplified set of major gasification reactions (Wang, T., 2017).

The reaction heats $\left(\Delta \mathrm{H}^{\circ}{ }_{\mathrm{R}}\right)$ are based on $298 \mathrm{~K}$ and $1 \mathrm{~atm}$, “+” indicates endothermic, and ""indicates an exothermic reaction. Heterogeneous reactions occur between a solid and either a gas or liquid, while homogeneous reactions happen within only the gas or liquid phase. Reactions [R1.8], [R1.9] and [R1.10] consist of a simplified two step thermal cracking gasification model (Wang, T., 2017). 
IGCC is less capital intensive and does not require large quantities of water when compared to DCL. Inversely, due to the nature of pyrolysis processing, gasification does not produce a high yield of heavy hydrocarbons like that of DCL. This is especially true when LRC is the main feedstock, as it is a hydrogen deficient carbon source needed to enhance the yield of pyrolysis oils. LRC's lack of hydrogen requires an outside source to increase the yield of tars. Methane is a preferred hydrogen donor for pyrolysis because it is $25 \%$ hydrogen by weight as well as cost efficient when compared to $\mathrm{H}_{2}$ gas. Methane, unfortunately, is a double-edged sword, as it has a notoriously high activation energy leaving it very stable at lower temperatures and increasingly difficult to convert into heavier hydrocarbons, let alone syngas. High amounts of heat are required to break the strong $\mathrm{C}-\mathrm{H}$ bonds present, even with recent catalyst developments. If unreformed, this remaining $\mathrm{CH}_{4}$ can dilute syngas and reduce the yield of value-added chemicals further along in the gasification process. With the use of catalysts to activate methane, the production of $\mathrm{H}_{2}$ still leaves solid carbon on the surface of the catalysts in the form of coke, effectively killing the catalyst until the coking is removed. The most common way of removing the coke from the outside of the catalysts used is by reacting the solid carbon with an oxidizing gas to form CO. Although oxygen would be an ideal choice as an oxidizing gas, partial oxidation with oxygen or air works too well, as it is an incredibly exothermic reaction. Its exothermic nature induces hot spots in the catalyst bed and insufficient heat removal rates that cause the operation to be difficult to control. Also, partial oxidation reactions require a cryogenic distillation unit to separate oxygen from air, further driving up operational costs when employing this method. "Light oxidants" such as $\mathrm{CO}_{2}$ are commonly used in the dry reforming process for the synthesis of syngas as it provides a safer, more controllable alternative for removing coking in the form of two CO molecules. 


\subsubsection{Coal Pyrolysis}

Coal gasification involves three main steps: thermal decomposition/pyrolysis, thermal cracking of the volatiles, and coal char gasification seen below in Figure 2.4.

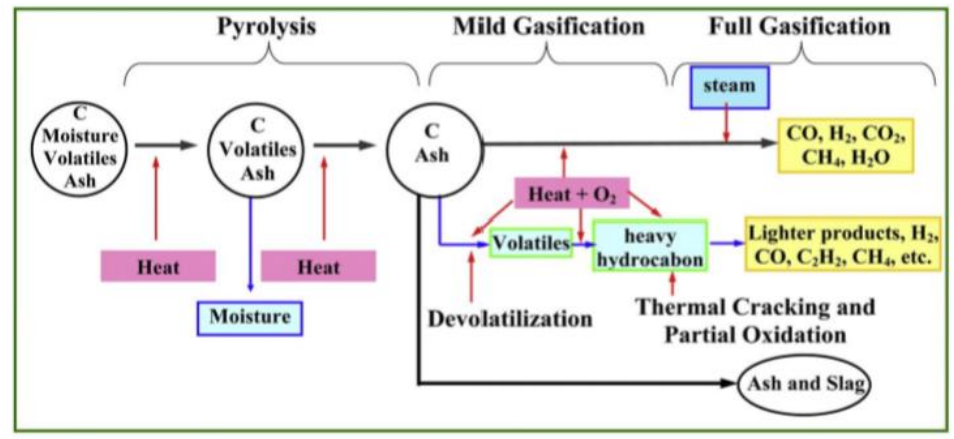

Figure 2.4: Simplified diagram of coal gasification (Wang, T., 2017)

Pyrolysis is a pretreatment for IGCC intended to release trapped moisture, devolatilize coal, and reduce the sulfur and tar content. Conventionally, pyrolysis uses low temperatures compared to complete combustion to de-volatize coal with a restriction of an external supply of oxygen or (other oxidizing gases) producing a mixture of devolatilized gases, tars, and a remainder of coal char. Devolatilization rate is influenced by temperature, pressure, residence time, particle size, and coal type. Coal pyrolysis involves two chemical processes: the decomposition of individual functional groups to produce light gas species and the decomposition of the macromolecular network producing smaller fragments that can derive into tar. Initially at $100{ }^{\circ} \mathrm{C}$, gases $\left(\mathrm{CO}_{2}, \mathrm{CH}_{4}, \mathrm{~N}_{2}\right)$ and moisture begin to desorb from the coal pores. Decomposition of individual functional groups begins at around $400{ }^{\circ} \mathrm{C}$ where a peak of low molecular weight organic solvents is released from the three-dimensional coal structure. The mechanics of pyrolysis are directly affected by the physical properties of coal char. Heating the char quickly often results in the reduction of its heat transfer coefficient, plateauing the temperature at around this $400{ }^{\circ} \mathrm{C}$ mark. At $500{ }^{\circ} \mathrm{C}$, fuel particles in their plastic state undergo a drastic change in their morphology, rehardening into char 
as the temperature reaches $550{ }^{\circ} \mathrm{C}$. As the temperature rises to around $\sim 750{ }^{\circ} \mathrm{C}$, a wide range of products can be seen as these primary solvents begin reacting with the coal matrix to produce other gases. This trend continues as the coal is heated over $1000{ }^{\circ} \mathrm{C}$ where eventually most volatile compounds have escaped the matrix, or the volatiles went into a plastic state, cracked, and redeposited themselves leaving char and slag as a carbon byproduct. Char yield decreases as a function of increasing temperature as fragments mobilize from the microstructure in the form of heavier volatiles or tars. The effect of increasing the temperature of LRC under different constant pressures shown in Figure 2.5. The reduction of char yield is inversely related with an increase in tar.

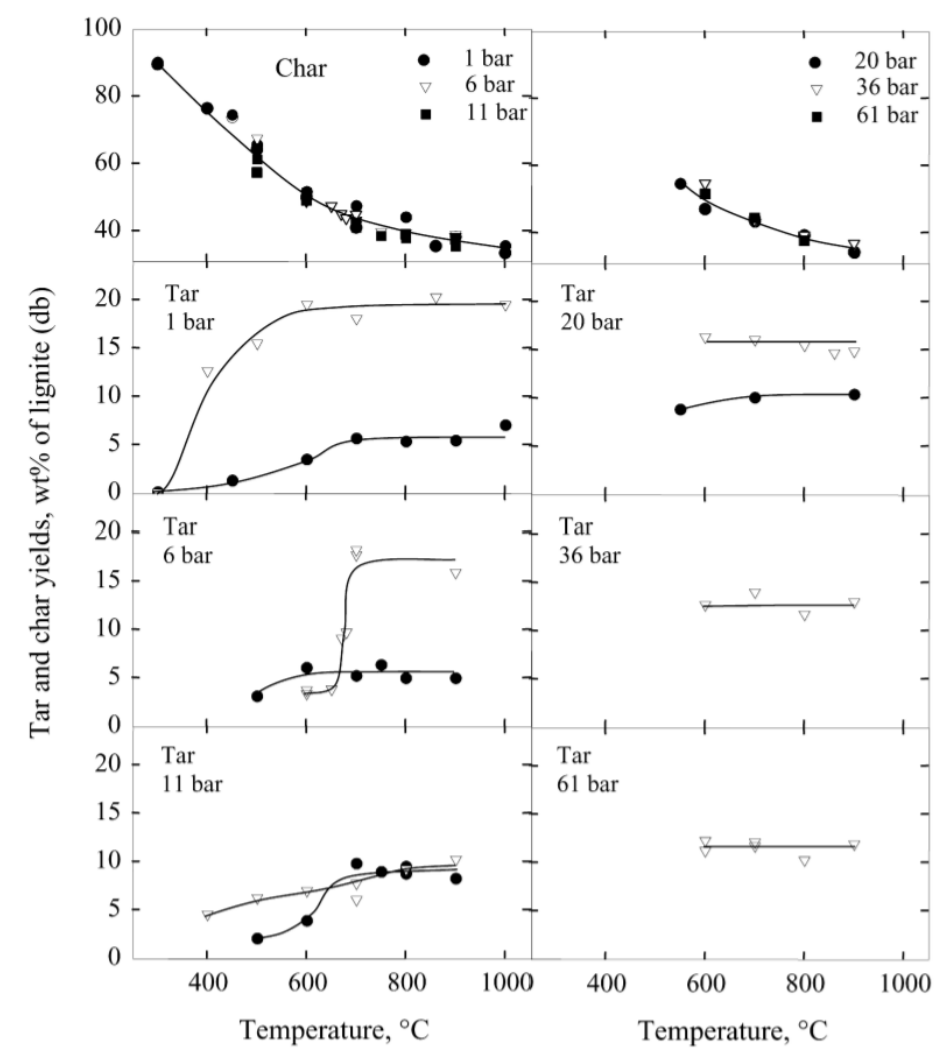

Figure 2.5: Effects of temperature, pressure and heating rate on char and tar yields from the pyrolysis of Loy Yang lignite (Sathe, Chirag, et al.)

Changes in pressure do not affect char yield, as it is primarily a function of temperature unlike tar yields. Temperatures above $600{ }^{\circ} \mathrm{C}$ and pressure held at 6 bar show the maximum of tar yields at 
approximately $16-20 \%$ where they begin to plateau. Char formation flattens out as the temperature increases, as only a limited amount of carbon atoms can be deposited on the surface on the char particles. Once the reaction nears completion, the char is removed as the volatile gasses and tars exit the top of the reaction vessel. LRC coal char contains traces of alkaline and alkaline earth metals within its matrix as well as other impurities such as trace sulfur and mercury compounds. Char can be used for a few applications such as a precursor to create activated carbon or simply recycled into the gasification process where it will undergo two endothermic, heterogenous reactions: $\mathrm{C}(\mathrm{s})+\mathrm{CO}_{2} \leftrightarrow 2 \mathrm{CO}[\mathrm{R} 1.2]$ carbon dioxide gasification and $\mathrm{C}(\mathrm{s})+\mathrm{H}_{2} \mathrm{O}(\mathrm{g}) \rightarrow \mathrm{CO}+\mathrm{H}_{2}$ [R1.3] steam-char gasification (Wang, T., 2017). Ultimately, char is currently a low-value solid byproduct of the gasification process with few applicable uses post-production in its raw form. Although char does not have many of the useful applications the rest of the gasification process provides, it does have several properties that exploit the hidden potential and increased marketability.

\subsubsection{Coal Char-Methane Reforming}

Coal char-methane reaction is where methane interacts with the solid char layer of the coal structure, separating $\mathrm{H}^{+}$ions from the molecular structure in a process called methane cracking. The hydrogen exits the reaction as a product, leaving the remaining carbon atoms on the surface of the char in the absence of an oxidizing agent.

$$
\mathrm{CH}_{4}=\mathrm{C}(\mathrm{s})+2 \mathrm{H}_{2} \quad \Delta \mathrm{H}^{\circ}{ }_{298 \mathrm{~K}}=74.6 \mathrm{~kJ} \mathrm{~mol}^{-1}
$$

LRC coal char as a methane catalyst has shown limited success for producing syngas. (Sun, ZhiQiang, et al.) used bituminous coal char for the char-methane reaction where a 9:1 ratio of $\mathrm{N}_{2} / \mathrm{CH}_{4}$ 
are flowed over mixtures of char and quartz between temperatures of $800{ }^{\circ} \mathrm{C}$ to $950{ }^{\circ} \mathrm{C}$ shown in Figures 2.6 and 2.7. The methane conversion is expressed:

$$
X=\frac{C_{C H_{4}, \text { in }}-C_{C_{4}, \text { out }}}{C_{C_{4}, \text { in }}}
$$

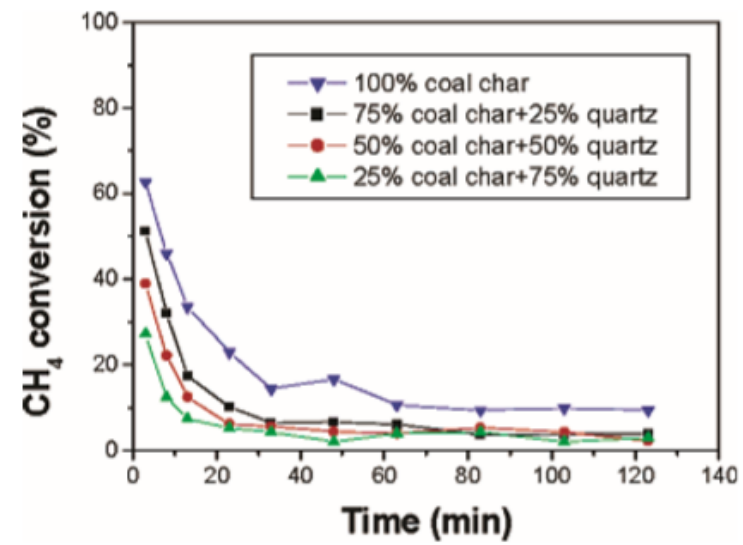

Figure 2.6: Methane cracking over a bed of various mixtures of raw char and quarts at 1173 K (Sun, Zhi-Qiang, et al.)

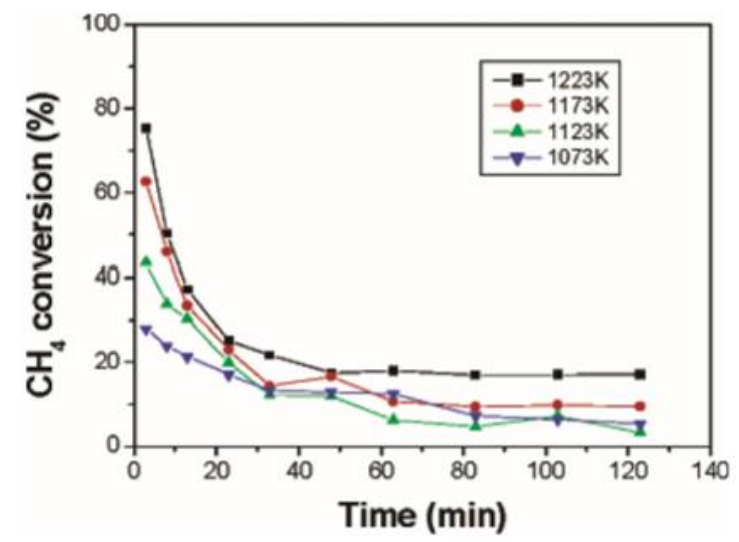

Figure 2.7: Methane conversions at various temperatures over bituminous coal char (Sun, Zhi-Qiang, et al.)

Despite char to quartz ratios or differing temperatures, all bituminous coal char samples lost most of their catalytic capabilities within the first 20 minutes of the reaction before flatlining. Figure 2.6 depicts that it is not just the high temperatures of the reactor that is causing the methane to crack, and the coal char is playing a large role in the reaction. The methane yield is lower with the test that introduces quarts mixed into the reactor. The reaction is dominated by the ratio of coal char and the use of high temperatures for the reaction that spikes the conversion of methane within the first 20 minutes. Since coal char's catalyst properties diminish quickly in its raw form, it would still be a preferable candidate for use in flash pyrolysis where the initial fuel is heated rapidly during the gasification process. Conventionally heating the coal char has clear limitations as shown, primarily from char having limited active sites on its own. Coal char can have a host of 
metal oxide impurities $\left(\mathrm{NaO}, \mathrm{SO}_{2}, \mathrm{MgO}, \mathrm{SiO}_{2}, \mathrm{Al}_{2} \mathrm{O}_{3}\right.$, etc.) that remain trapped in the polymerized matrix post-pyrolysis. Several of these oxides have shown their capabilities in dry reforming to produce syngas, but this process requires the addition of $\mathrm{CO}_{2}$ to react with coking that forms on nearly all the active surfaces. The above reaction of char and methane was potentially stifled quickly as the methane was rapidly oxidized. As methane is completely oxidized, it leaves the remaining solid carbon to coat the surfaces what the leftover metal oxides that could have been the main contributors to the reaction.

LRC char could be treated, altered, or preferably used as a support for another catalytic particle to enhance these catalytic capabilities for long term use while maintaining its MW absorbing properties. This would increase the reaction life and save the amount of char regenerations as carbon is eroded form the inexpensive char catalyst. The utilization of coal char as a heat syncing/catalytic support would expand its marketable viability as a syngas producing catalyst. In its baseline form, LRC coal char is shown to be short-lived when cracking methane gas. LRC coal char does show the potential to react, albeit briefly, with methane and carbon dioxide gas as well as being reliable with MW bulk bed heating.

\subsubsection{Pyrolysis Optimizations and Improvements}

Coal pyrolysis is considered a free radical process where covalent bonds are cleaved, and secondary reactions can occur as radical fragments are also removed for the surface of LRC coal char. These fragments combine with free radicals to create coal tars. Unfortunately, due to insufficient amounts of hydrogen found in coal, the yield of these coal tars and pyrolysis gases are poor (Zhang, Xu, Zhao, \& Liu, 2007). 


\subsubsection{Hydropyrolysis}

Hydropyrolysis is where typical pyrolysis processes are supplemented with a $\mathrm{H}_{2}$ atmosphere which increases tar yields as well as the overall quality of the coal tar (Zhu P. et al.). Although effective, hydrogen is expensive to buy or produce, limiting the viability of it being used as a proper hydrogen donor. Methane as well as other gases such as $\mathrm{CO}_{2}$ and $\mathrm{CO}$ have been researched thoroughly to provide an answer to increased coal tar yield and quality. Methane has a high hydrogen to mass ratio, is readily available, and relatively inexpensive when compared to hydrogen. $\mathrm{CH}_{4}$ when dehydrogenated dissociates into $\mathrm{H}^{+}$and $\mathrm{CH}_{\mathrm{x}}{ }^{*}$ radicals that can participate in the pyrolysis reaction. $\mathrm{CO}_{2}$ has been shown to promote the cracking of benzene rings, methyl, and methyl groups, as well as decreasing the interactions between $\mathrm{H}$ and char. $\mathrm{CO}_{2}$ also participates in the removal of deposited carbon in the form of coke on catalytic particle, extending their reactionary lifespan and producing a viable source of $\mathrm{CO}$ gas that can also participate in the pyrolysis reactions.

\subsubsection{Pyrolysis Catalysts}

LRC pyrolysis has also shown favorable results with the addition of various heterogenous catalysts improving the yield and quality of tars and pyrolysis gases. In an ideal world, the use of platinum metals has been shown to be excellent catalysts for a plethora of reactions and shown high resistance to deactivation, but they are all incredibly expensive. Due to the current predictable yields, the use of high efficiency catalysts such as platinum, palladium, rhodium, and rubidium are out of the question, leaving the door wide open for the use of other catalysts. Alkaline earth and transition metal catalysts have been at the forefront of catalyst development due to their low costs, availability, and affordable recovery while maintaining efficient catalytic results. Their use in pyrolysis is not an unfamiliar practice, a variety of different catalysts have been explored to 
improve the yield and quality of coal tars and profitable volatile gases depending on the desired design. While these cheaper catalysts are much more economically viable, they are not as resistant to carbon deposits like platinum metal catalysts which has a staggering resistance to coking in comparison (Devendra Pakhare et al.). Although these metals and metal oxides are more economically viable, they come with drawbacks not typically seen in with platinum group metals. Transition metals Ni and Fe have been cited in numerous studies on their catalytic capabilities to weaken/break the $\mathrm{C}-\mathrm{H}$ bonds in methane gas as well as assist in increasing the yield of higher quality pyrolysis coal tars at the cost of being deactivated relatively quick. For example, Ni based catalysts prepared on various supports all exhibit this deactivation at some point where the reaction rate begins to dip as the catalysts experience the coking effects from methane activation. The addition of platinum group metals has been shown to mitigate and sometimes even eliminated this coking effect when these transition metals are promoted by a noble metal like platinum and rhodium (Devendra Pakhare et al.). It's these three properties: Anti-coking, sufficient methane activation, and affordability that have driven research to develop and test numerous catalysts in search of the ideal candidate for improved pyrolysis, hydropyrolysis, and DRM.

\subsubsection{Microwave Pyrolysis of LRC}

Conventional pyrolysis of LRC using traditional heating can be considered less than desirable due to its low heating value. In recent years, the study of MWs as a thermal source have been advantageous as it exploits the naturally MW-absorbing capability of LRCs. MWs are an electromagnetic wave with a frequency range of $300 \mathrm{MHz}-300 \mathrm{GHz}$, which has been used in the past for lignite drying, biomass pyrolysis, as well as other processes (Longzhi et al., 2018). The high moisture and inorganic minerals have high dielectric properties that absorb MW energy thus heating the bulk coal uniformly during pyrolysis. Furthermore, LRC has a high ash content that 
could also contribute catalytically to the conversion of methane into value-added chemicals. MW heating stipulates an excellent opportunity to optimize the performance of catalysts according to processing characteristics. MW heating offers well established advantages when compared to conventional methods, including instantaneous heating the bulk beds with precise temperature control, selective and uniform heating, reduced energy consumption, and process diversity (Farag et al.). MW pyrolysis can be considered superior to traditional heating methods for LRC as MW heating has the additional benefits of significantly lower power consumption and reductions in capital and operational costs. Even if the LRC coal char is devoid of moisture and most of the sulfur containing compounds were removed, LRC's alkaline and alkaline earth metals trapped within the coal matrix also absorb MW radiation. The absorption of MW radiation is only proportional to the number of impurities and moisture left in the coal char which will vary depending on the pyrolysis conditions and where the coal was sourced respectively. MW-assisted coal pyrolysis has room for optimization, mainly contributing to the temperatures in which experiments are conducted. Temperatures needed for methane to begin interacting with the LRC are still north of $700{ }^{\circ} \mathrm{C}$ where some of the heavier hydrocarbons can vaporize, react, and leave the system in the form of lighter gases. Maximizing the formation of heavier hydrocarbons would require a lower temperature below that of approximately $500{ }^{\circ} \mathrm{C}$. In theory, by adding metal oxide particles to LRC coal char bed, the particles can assist in heat distribution and add activation sites for the methane/coal char reaction to take place at lower temperatures. The combination of cost saving opportunities coupled with the useful MW absorbing capabilities of LRC coal char and metal oxide catalysts have pushed MW pyrolysis to the forefront of LRC upgrading research as a potential future of low emission fuel synthesis manufacturing. 


\subsubsection{Hydropyrolysis and Methane Cracking Catalysts}

Heterogeneous catalysts are fundamental to the foundation of the chemical and energy industries as a centralized science for the transitioning of a carbon positive footprint to a carbon neutral one. Heterogeneous catalysts are solid particles or surfaces that react with the liquid or gaseous reactants, contrasting homogeneous catalysts that exist in the same phase as the reactants. They rely on interfacial boundary interactions between a solid phase catalyst and gaseous reactants, such as what is seen during hydropyrolysis and DRM. At high temperatures $\left(>630{ }^{\circ} \mathrm{C}\right)$, methane activation is understood to frequently involve methyl groups and methyl radicals although the exact reaction mechanisms are still widely debated. Therefore, the $\mathrm{C}-\mathrm{H}$ bond $(434 \mathrm{~kJ} / \mathrm{mol})$ is what is primarily focused upon for comparison. The non-catalytic deprotonation reaction of gaseous methane has the standard enthalpy of reaction $\sim 1745 \mathrm{~kJ} / \mathrm{mol}$ forming a methyl anion and a proton. The high temperature requirements for noncatalytic methane conversion requires harsher conditions, such as with the oxidative coupling of methane (OCM) where the temperature requirement is not as high, but the overall process is performed at $>2.5$ bar. Thermodynamic data suggests that methane begins to become unstable around $500{ }^{\circ} \mathrm{C}$, however, conversion at these temperatures remains exceedingly low until $950{ }^{\circ} \mathrm{C}$ (at 1 bar). Typical noncatalytic hydropyrolysis plants operate at temperatures north of $1200{ }^{\circ} \mathrm{C}$ to insure high activation and sustainable reaction rates.

\subsubsection{Methane Upgrading - Catalytic Interactions}

Despite the challenges presented of activating methane, there is significant progress in the direct conversion of methane into hydrocarbons, using oxidative coupling, dry/steam reforming, and utilizing hundreds of various metal and metal oxide particles as catalysts. One of the most studied 
phenomena is the activation of methane over Lewis-Base pairs, which plays an essential role in the attraction of weakly acidic methane gas as depicted in Figure 2.8.

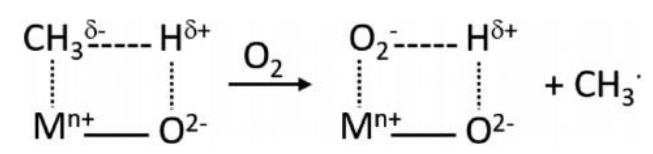

Figure 2.8: The superoxide radical $\mathrm{O}^{2-}$ of a metal oxide adsorbing a proton of $\mathrm{CH}_{4}$ in close vicinity (Schwach, Pan, \& Bao, 2017)

If the $\mathrm{H}^{+}$or $\mathrm{CH}_{3}$ adsorbs alone on the surface of the metal oxide particle, they preferentially adsorb as a Lewis base onto oxygen atom. The oxygen atom is slightly electronegative while the rest of the metal is slightly electropositive. The oxygen forms a hydroxyl group with the hydrogen atom or the methyl radical, unless they are coadsorbed, where one site will function as a Lewis base while the other will act as a Lewis acid site. This case produces the acid-base pair where the metal cation pulls on the now slightly electronegative half of the methane radical, lowering the activation energy of the $\mathrm{C}-\mathrm{H}$ bond further until the $\mathrm{H}^{*}$ is cleaved away from the new methyl radical. This is the best-case scenario, as it results in the lowest dissociative activation energy for methane. Eventually this $\mathrm{H}^{+}$will desorb and recombine with another like $\mathrm{H}^{+}$radical or cleave $\mathrm{H}^{+}$from other methane molecules. This abundance of free radicals' forms what is called a "radical pool," where the reactions between the inflowing gas is activated, producing free radicals that react with other 
methane particles to continue producing more free radicals. The chain reaction of radical production is ongoing until it is stopped by the end user.

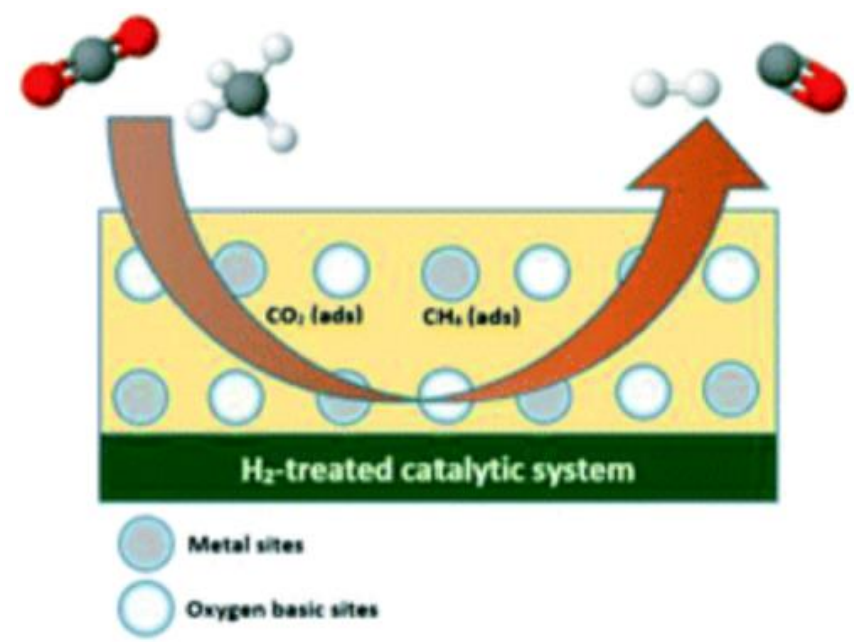

Figure 2.9: Illustration of reforming gasses adsorbing and reacting with metal oxide basic sites (Aziz, Jalil, Wongsakulphasatch, \& Vo, 2020)

These methyl radical pools of can produce heavier hydrocarbon, particularly aromatic, products in non-oxidative conditions when compared to OCM and like-processes. Although aromatic formation of methane in the absence of oxygen is not thermodynamically favorable at low temperatures $\left(<500{ }^{\circ} \mathrm{C}\right)$, the equilibrium methane conversion at $700{ }^{\circ} \mathrm{C}$ is $\sim 12 \%$. At this temperature, benzene, and naphthalene, in approximately equimolar amounts, are the thermodynamically preferred products (Wang et al., 1997). It is generally agreed upon that materials with significant quantities of Lewis/Bronsted acid sites have several significant mechanisms in methane dearomatization (MDA). First reports of MDA were recorded using a molybdenum catalyst supported on a HZSM-5 zeolite structure $\left(\mathrm{MoO}_{3} / \mathrm{HZSM}-5\right)$ within a fixed bed reactor that produced a $7 \%$ conversion of methane to benzene with a selectivity of $100 \%$ (Wang et al., 1993). Various investigations of the Mo/HZSM-5 catalysts thereafter have also confirmed methane conversions of 5-8\% with selectivities of benzene between $\sim 65-70 \%$ (Solymosi et al., 1995, 1996, 1997; Szöke \& Solymosi, 1996, Wang et al., 1996, 1997). It is 
theorized that the formation of benzene specifically is because of the zeolite framework and how the Mo species anchor themselves to the porous structure. The Bronsted active sites serve as these anchoring points for the Mo species in and around the pores of the zeolite structure. In this case, they are not known to play a significant role in MDA, instead acting as the necessary scaffolding for the Mo catalytic particles to react with methane. The location of methane activating sites are located with the pores and channels of the zeolite (Zhang et al., 1999). During catalyst preparation, the Mo particles infiltrate the zeolite structure forming $\mathrm{Al}-\mathrm{O}-\mathrm{Mo}$ linkages within the pores $(\mathrm{Hu}$ et al., 2009; Zheng et al., 2008). Particularly, these sites, the size, and shape of these channels are what are thought to influence the formation of benzene rings. The ratio of $\mathrm{Si} / \mathrm{Al}$ in the zeolite determines the amount of Bronsted active sites and in turn the size and shape of the pores (Schwach, P. et al., 2017). Rice, Chakraborty, \& Bell, 1999 noted that low Si/Al ratios (<15), have the optimal concentration of acidic sites for Mo stabilization within the porous structure. Mo is most likely to stabilize on two $\mathrm{AlO}_{4}{ }^{-}$centers at these concentrations, providing the necessary $\sim 0.55$ $\mathrm{nm}$ distance between $\mathrm{Mo}(=\mathrm{O})_{2}{ }^{2+}$ molecules within the zeolite pores for benzene formations (Rice, Chakraborty, \& Bell, 1999). The initial anchoring of the $\mathrm{MoO}_{3}$ is not what initiates the MDA process. MDA with Mo/HZSM-5 catalysts have an induction period prior to the formation of benzene molecules, where the inlet methane partially carburizes the Mo into molybdenum oxycarbide $\left(\mathrm{MoO}_{\mathrm{x}} \mathrm{C}_{\mathrm{y}}\right)$, releasing $\mathrm{CO}, \mathrm{CO}_{2}$, and $\mathrm{H}_{2} \mathrm{O}$ as products (Liu, Wang, Ohnishi, \& Ichikawa, 1999). The $\mathrm{MoO}_{\mathrm{x}} \mathrm{C}_{\mathrm{y}}$ species can convert $\mathrm{CH}_{4}$ into $\mathrm{C}_{2+}$ products, but as the oxycarbide species is fully carburized into molybdenum carbide $\left(\mathrm{MoC}_{\mathrm{x}}\right)$, benzene formation can finally occur withing the zeolite pores (Lezcano-González et al., 2016). Xing et al., 2010 and Zhou et al., 2012 suggested that the carbidic molybdenum is first hydrogenated to form molybdenum carbene and methylene bridge (in the case of dimeric species). They suggested that these could be involved with the 
creation/production of ethylene during the intermediate reaction. An illustration of the reactionary pathway for ethylene formation over the Mo/HZSM-5 catalyst is seen below in Figure 2.10.
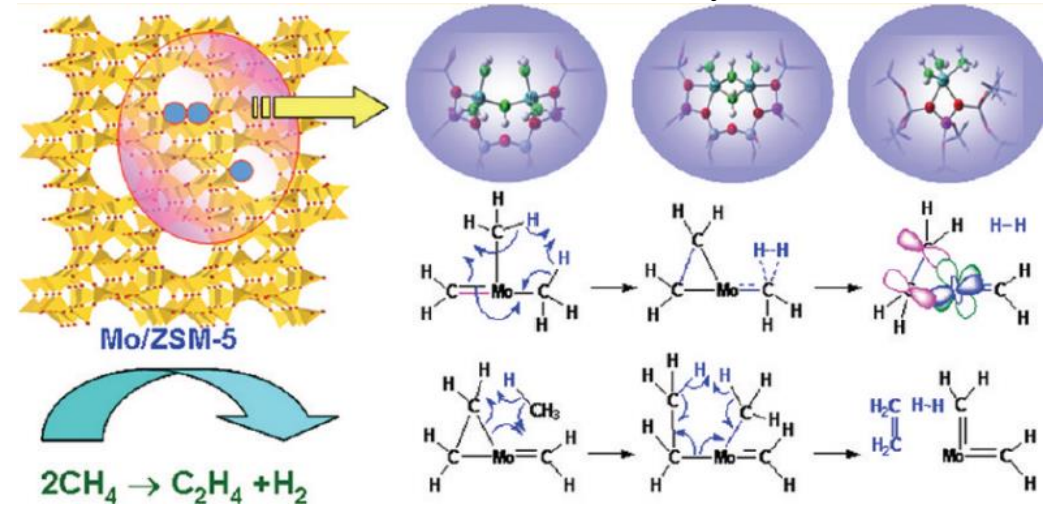

Figure 2.10: Mo/HZSM-5 zeolite catalysts and the reaction mechanisms of methane

dehydrogenation and coupling to ethylene (Zhou et al., 2012)

$\mathrm{Mo}=\mathrm{CH}_{2}$ represents a Lewis acid-base pair, polarizing and activate methane to form a negatively charged methyl group bound to the Mo cation and a positively charged methyl group is formed by reaction of the carbene with the resulting proton from the $\mathrm{C}-\mathrm{H}$ bond scission. The two $\mathrm{CH}_{3}$ groups then undergo $\mathrm{C}-\mathrm{C}$ coupling, eliminating $\mathrm{H}_{2}$ to form an ethylene ligand. The ethylene group desorbs after the activation of a third methane molecule to replace the original carbene ligand incorporated in the formed ethylene. Ethylene then reacts further to benzene in the zeolite channels via successive hydrogenation/dehydrogenation reactions catalyzed by Brønsted acid sites.

Other pyrolysis processes have also depicted these free radicals stabilize by combining with other free radicals to form volatiles including tars and gas products. While these reactions are still theoretically possible at high temperatures during the hydropyrolysis reaction, the yield of heavier hydrocarbons typically found as tars and oils is virtually zero as they are dissociated into lighter hydrocarbons at such temperatures. The use of catalysts during hydropyrolysis and dry reforming are integral to optimization, because they procure the reduction of reaction temperatures within quintessential working ranges for pyrolysis oils and tars to stabilize and not be incinerated by the 
extreme environment providing both energy cost savings and improved product marketability. Research and experimentation on these interactions with metal oxide surfaces and methane are seen in abundance. Popular examples of these metal oxides that have been at the forefront of many studies are alkaline earth metal oxides such as $\mathrm{MgO}, \mathrm{CaO}$, and $\mathrm{SrO}$. Alkaline earth metal oxides are particularly popular catalysts for OCM reactions for their ability to form Lewis base pairs with acidic gases. Alkaline earth metal oxides have relatively low electro negativities, are readily available, are economically viable, and have readily been theorized and tested to show their capability of making these Lewis base-pairs with methane. $\mathrm{MgO}$ has undergone extensive studies as a viable methane activation catalyst due to the abundance of basic sites on its surface. $\mathrm{Ni} / \mathrm{MgO}$ catalysts demonstrated an increase in coal tar yields by $40 \sim 80 \%$ when used in the presence of pyrolysis gases (PG) when compared to both $\mathrm{N}_{2}$ and $\mathrm{PG}$ respectively at temperatures between 550$600{ }^{\circ} \mathrm{C}$ (Zhu et al.). The composition of the tar also improved with the addition of $\mathrm{Ni} / \mathrm{MgO}$ packed after the pulverized coal, promoting the formation of maltene opposed to asphaltene due to the higher amount of light hydrocarbon fractions created during the hydropyrolysis reaction seen in Figure 2.11 (Zhu et al.). 


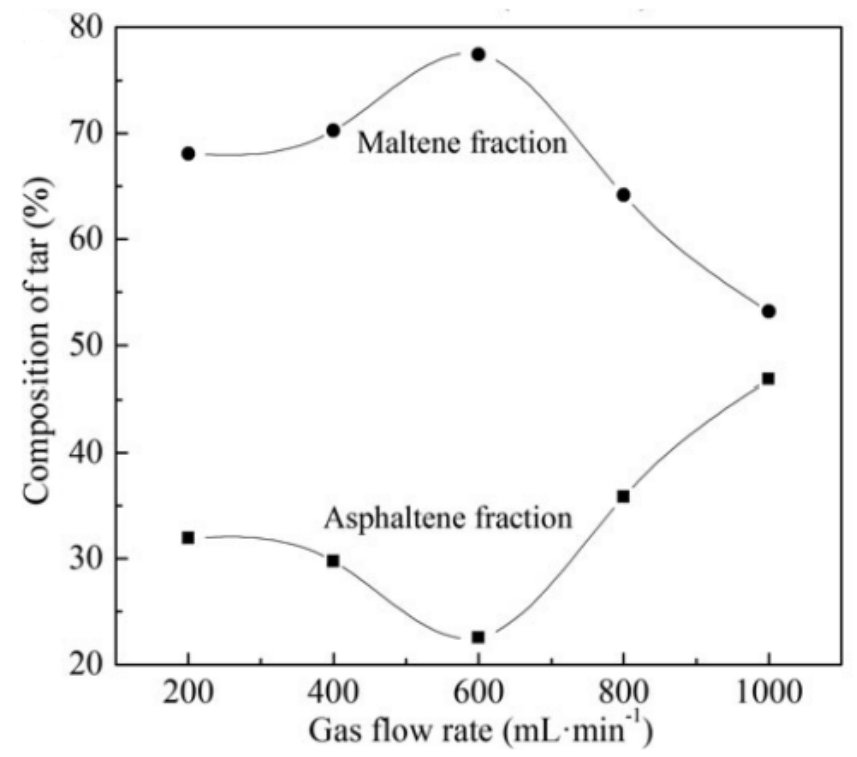

Figure 2.11: Effect of gas flow rate on production yield under $\mathrm{N}_{2}, \mathrm{PG}$, and $\mathrm{PG}$ over $\mathrm{Ni} / \mathrm{MgO}$ on composition of tar (Zhu et al.).

Similar trends were found when reacting coal under $\mathrm{CO}_{2} / \mathrm{CH}_{4}$ environments with a $\mathrm{Ni} / \mathrm{MgO}$ catalyst, and instead they found even higher yields of coal tar peaking $~ 34 \%$ at temperatures of $800{ }^{\circ} \mathrm{C}$ (Liu et al.). Pure $\mathrm{MgO}$ hydrothermal treated and synthesized via sol-gel synthesis supports the theory of the methane adsorption on its surface, as these synthesis methods yielded the highest adsorption levels of both pure methane and $\mathrm{CH}_{4}$ and $\mathrm{CO}$ when compared to other synthesis methods and laboratory grade $\mathrm{MgO}$ (Schwach et al.). Synthesis, calcination, and surface morphology of $\mathrm{MgO}$ were the main determining factors when observing gas adsorption. 

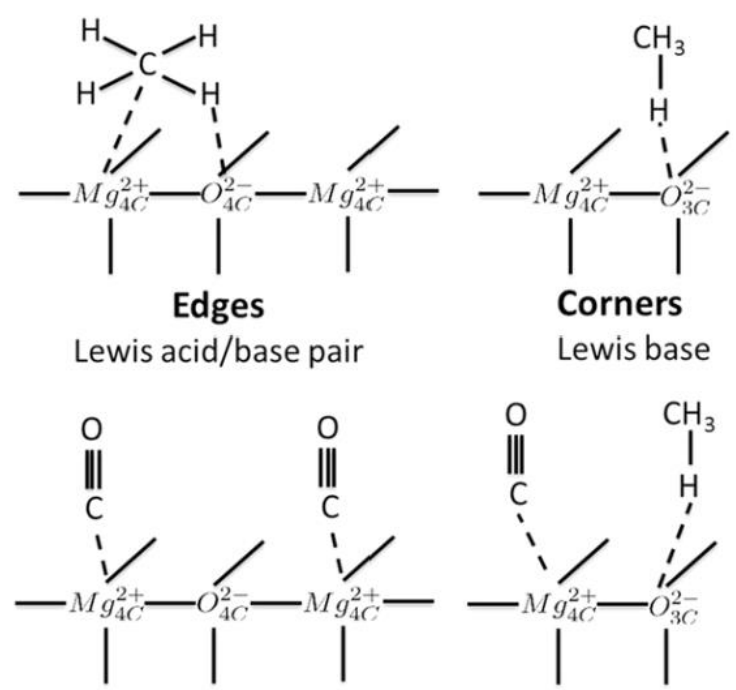

Figure 2.12: Methane adsorption and coadsorption of $\mathrm{CO}$ and $\mathrm{CH}_{4}$ on $\mathrm{MgO}$ edges and corners.

(Schwach et al.)

A $\mathrm{CaO}$ particle's surface also exhibits similar tendencies when placed within a methane environment. Alkaline earth metal catalysts possess similar properties, each form temporary $\mathrm{OH}-$ states on $\mathrm{O}^{2-}$ active sites while $\mathrm{CH}_{3}{ }^{+}$is attached to the alkaline earth metal (Maiti G.). Once separated, these adsorbed ions could combine with like species near them on the oxide lattice to form $\mathrm{C}_{2} \mathrm{H}_{6}$ and $\mathrm{H}_{2}$ gas and/or react with other desorbed radicals or unreacted gases entering the system.

\subsection{Dry Reforming Methane (DMR)}

Natural gas reforming technologies have been extensively studied to convert methane into liquid fuels or heavier hydrocarbon such as formaldehyde, benzene, propanol, methanol, and other aromatics, but unfortunately these processes have low yields or are infeasible for processing on an industrial scale. Three processes reign supreme when considered for industrial scale for methane reforming: steam reforming, partial oxidation with oxygen or air, and DMR with carbon dioxide. The fundamental approach of the DMR process is to convert methane and carbon dioxide into 
valuable synthesis gases, $\mathrm{H}_{2}$ and $\mathrm{CO}$, reducing undesirable secondary reactions in the gas phase and maintaining a high selectivity of syngas. The complete reactionary mechanism pathways for DRM are seen below in Table 2.1 (Nikko and Aman, 2011).

Table 2.1: The complete reactionary mechanism pathways for DRM (Nikko and Aman, 2011)

Reaction \# Reaction $\quad \Delta \mathrm{H}^{298}(\mathrm{~kJ} / \mathrm{mol})$

$1 \quad \mathrm{CH}_{4}+\mathrm{CO} \leftrightarrow \mathrm{CO}+2 \mathrm{H}_{2}$

$2 \mathrm{CO}_{2}+\mathrm{H}_{2} \leftrightarrow \mathrm{CO}+\mathrm{H}_{2} \mathrm{O}$

$3 \quad 2 \mathrm{CH}_{4}+\mathrm{CO}_{2} \leftrightarrow \mathrm{C}_{2} \mathrm{H}_{6}+\mathrm{CO}+\mathrm{H}_{2} \mathrm{O} \quad 106$

$4 \quad 2 \mathrm{CH}_{4}+2 \mathrm{CO}_{2} \leftrightarrow \mathrm{C}_{2} \mathrm{H}_{4}+2 \mathrm{CO}+2 \mathrm{H}_{2} \mathrm{O}$

$5 \quad \mathrm{C}_{2} \mathrm{H}_{6} \leftrightarrow \mathrm{CH}_{4}+\mathrm{H}_{2} \quad 136$

$6 \quad \mathrm{CO}+2 \mathrm{H}_{2} \leftrightarrow \mathrm{CH}_{3} \mathrm{OH}$

$7 \quad \mathrm{CO}_{2}+3 \mathrm{H}_{3} \leftrightarrow \mathrm{CH}_{3} \mathrm{OH}+\mathrm{H}_{2} \mathrm{O}$

$8 \quad \mathrm{CH}_{4} \rightarrow \mathrm{C}+2 \mathrm{H}_{2} \longrightarrow 74.9$

$9 \quad 2 \mathrm{CO} \rightarrow \mathrm{C}+\mathrm{CO}_{2} \quad-172.4$

$10 \quad \mathrm{CO}_{2}+2 \mathrm{H}_{2} \leftrightarrow \mathrm{C}+2 \mathrm{H}_{2} \mathrm{O} \quad-90$

$11 \quad \mathrm{H}_{2}+\mathrm{CO} \leftrightarrow \mathrm{H}_{2} \mathrm{O}+\mathrm{C} \quad-131.3$

$12 \mathrm{CH}_{3} \mathrm{OCH}_{3}+\mathrm{CO}_{2} \leftrightarrow 3 \mathrm{CO}+3 \mathrm{H}_{2}$

$13 \quad 3 \mathrm{H}_{2} \mathrm{O}+\mathrm{CH}_{3} \mathrm{OCH}_{3} \leftrightarrow 2 \mathrm{CO}_{2}+2 \mathrm{H}_{2} \quad 136$

$14 \mathrm{CH}_{3} \mathrm{OCH}_{3}+\mathrm{H}_{2} \mathrm{O} \leftrightarrow 2 \mathrm{CO}+4 \mathrm{H}_{2}$ 
Conversion of $\mathrm{CH}_{4}$ and $\mathrm{CO}_{2}$ individually possess relatively lower activation energies when compared to the activation of both in the same reaction, but the combination of the two are typically seen in natural gas, where the conversion of the two have significant implications over the utilization of natural gas (Cai \& Hu, 2019). Reforming methane in this manner offers valuable environmental benefits including biogas utilization, removal of greenhouse gases (GHG), and the conversion of these GHGs into profitable syngas (Usman, Wan Daud, \& Abbas, 2015). DMR is typically operated between temperatures of $700-950{ }^{\circ} \mathrm{C}$ where these high temperatures can lead to secondary gas phase reactions. However, it was also found that both $\mathrm{CO}_{2}$ and $\mathrm{CH}_{4}$ react more readily at higher temperatures due to their overall endothermic reaction, thus creating a delicate balance between high conversion the selectivity of syngas. The activation energies of the $\mathrm{C}-\mathrm{H}$ and $\mathrm{C}-\mathrm{O}$ bonds in $\mathrm{CH}_{4}$ and $\mathrm{CO}_{2}$, respectively, offer a great challenge with their incredible stabilities and require efficient catalysts to activate (Cai \& Hu, 2019). Further complicating DMR's commercial viability and environmental benefits it displays, the reaction suffers from carbon deposits from methane de-hydrolyzation and disproportionate $\mathrm{CO}$ formation from this carbon reacting with $\mathrm{CO}_{2}$, otherwise known as the reverse Boudouard reaction. Carbon disposition can be estimated by the ratios of oxygen to carbon and hydrogen to carbon in the feed gas. Lower ratios of these gases are what leads to increased carbon deposition that quickly stifles the reaction as carbon continues to accumulate. Theoretical studies on the thermodynamics of the DMR reaction 
suggest that the reaction does not begin until surpassing a minimum temperature of $640{ }^{\circ} \mathrm{C}$, however, undesirable side reactions are typically seen until $700{ }^{\circ} \mathrm{C}$. Other studies suggest the temperature should be slightly higher at $750{ }^{\circ} \mathrm{C}$ to mitigate side reactions and increase the $\mathrm{H}_{2}$ yield, or the ratio of $\mathrm{CH}_{4} / \mathrm{CO}_{2}$ should be increased to reduce carbon deposition. Unfortunately, increasing the ratio of $\mathrm{CH}_{4} / \mathrm{CO}_{2}$ has additionally been shown doing the opposite and depositing more carbon while adding unnecessary complexity to the process by requiring methane capturing capital to recover unreacted methane. Most catalysts currently for DRM are earth abundant transition metals and noble metals (Cai \& Hu, 2019). Earth abundant transition metals are low in cost and provide high conversion rates, but usually are prone to high coking rates. Noble metals on the other hand have the opposite problem, offering high anti-coking properties, but are not economically viable. DRM is excellent, in theory, with the promise of high conversion of GHGs methane and carbon dioxide into relevant fuel sources while mitigating the formation of undesirable products, but the process is riddled with challenging obstacles that hold it back from being at the forefront of synthesis gas production. The need for an affordable, carbon-resistant catalyst is imperative to properly optimize DRM into becoming a commercially viable syngas manufacturing process.

\subsubsection{DRM Catalysts}

Research into DRM and DRM-like processes have undergone countless studies observing the effects of varied catalyst designs and their resistivity to carbon deposition. In industry, a favorable catalyst for DRM should include the following features: good catalytic activity, high stability, a readily available resource, and low cost (Longzhi Li, 2018). Noble metal catalysts have shown some of the most promising results, as they have shown superior coking resistances, high stability, and increased activity at high temperatures when compared to other popular catalyst candidates (Djinović, Črnivec, Batista, Levec, Pintar, 2011). One study tested noble metals (Rh, Ru, Pt, Pd, 
and Ir) at $5 \mathrm{wt} \%$ supported with alumina compared to $\mathrm{Co}$ and $\mathrm{Ni}$ at $10 \mathrm{wt} \%$ concluding the noble metal catalysts displayed higher stability and coking resistance when compared to the transition metals on the same supports (Hou, Chen, Fang, Zheng, Yashima, 2006).

Table 2.2: Activity of different metals for $\mathrm{CH}_{4}$ reforming with $\mathrm{CO}_{2}$ (Hou, Chen, Fang, Zheng, Yashima, 2006)

\begin{tabular}{|c|c|c|c|c|c|}
\hline \multirow[t]{2}{*}{ Catalysts } & \multicolumn{2}{|c|}{ Initial conv. $(\mathrm{mol} \%)^{\mathrm{a}}$} & \multicolumn{2}{|c|}{ Final conv. $(\mathrm{mol} \%)^{\mathrm{b}}$} & \multirow{2}{*}{$\begin{array}{l}\left.\text { Deposited Carbon }{ }^{\mathrm{c}}\right) \\
\text { (mg coke/g cat h) }\end{array}$} \\
\hline & $\mathrm{CH}_{4}$ & $\mathrm{CO}_{2}$ & $\mathrm{CH}_{4}$ & $\mathrm{CO}_{2}$ & \\
\hline $\mathrm{Ni}(10 \mathrm{wt} \%) / \alpha-\mathrm{Al}_{2} \mathrm{O}_{3}{ }^{\mathrm{f}}$ & 71.5 & 77.1 & 67.4 & 73.7 & 24.0 \\
\hline $\mathrm{Co}(10 \mathrm{wt} \%) / \alpha-\mathrm{Al}_{2} \mathrm{O}_{3}$ & 56.0 & 66.0 & 36.9 & 50.3 & 49.4 \\
\hline $\mathrm{Ru}(5 \mathrm{wt} \%) / \alpha-\mathrm{Al}_{2} \mathrm{O}_{3}$ & 39.2 & 51.9 & 36.9 & 63.8 & 0.0 \\
\hline $\mathrm{Rh}(5 \mathrm{wt} \%) / \alpha-\mathrm{Al}_{2} \mathrm{O}_{3}$ & 57.2 & 64.4 & 56.9 & 63.8 & 0.0 \\
\hline $\mathrm{Pt}(5 \mathrm{wt} \%) / \alpha-\mathrm{Al}_{2} \mathrm{O}_{3}$ & 25.6 & 31.1 & 11.0 & 17.0 & 0.0 \\
\hline $\mathrm{Pd}(5 \mathrm{wt} \%) / \alpha-\mathrm{Al}_{2} \mathrm{O}_{3}$ & 38.8 & 41.2 & 12.7 & 15.8 & 4.9 \\
\hline $\mathrm{Ir}(5 \mathrm{wt} \%) / \alpha-\mathrm{Al}_{2} \mathrm{O}_{3}$ & 25.6 & 38.1 & 26.6 & 39.3 & 0.0 \\
\hline $\mathrm{Rh}(5 \mathrm{wt} \%) / \mathrm{Yas} 3-8^{\mathrm{g}}$ & 84.4 & 85.2 & 84.8 & 85.5 & 0.0 \\
\hline
\end{tabular}

Only $\mathrm{Pd} / \alpha-\mathrm{Al}_{2} \mathrm{O}_{3}$ showed light depositions of carbon (4.9 $\mathrm{mg}$ coke/g cat $\mathrm{h}$ ) between the noble metals due to assumed sintering at higher reaction temperatures while the remaining noble metals displayed no evidence of such carbon deposited. The catalytic and stabilities trend of the catalysts was $\mathrm{Rh} / \alpha-\mathrm{Al}_{2} \mathrm{O}_{3}>\mathrm{Ru} / \alpha-\mathrm{Al}_{2} \mathrm{O}_{3}>\mathrm{Ir} / \alpha-\mathrm{Al}_{2} \mathrm{O}_{3}>\mathrm{Pd} / \alpha-\mathrm{Al}_{2} \mathrm{O}_{3}>\mathrm{Pt} / \alpha-\mathrm{Al}_{2} \mathrm{O}_{3}$. Ni and $\mathrm{Co}$ had initial conversions of $71.5 \%$ and $56.0 \% \mathrm{CH}_{4}$ conversion and $77.1 \%$ and $66.0 \% \mathrm{CO}_{2}$ conversions, respectively, but after 240 minutes both metals' conversions rates fell significantly due to large amounts of coking. Co had the highest amounts of coking (49.4 mg coke/g cat h) while Ni had about half that value (24.0 $\mathrm{mg}$ coke/g cat $\mathrm{h}$ ), both being common occurrences with non-noble metal catalysts undergoing DRM reactions. Another study reported high coking resistances and catalytic stability with noble metals incorporated over $\mathrm{Mg}$ - Al layered double hydroxides where catalytic activity was directly related to the resistance of carbon deposition. $\mathrm{Rh} / \mathrm{MgAlO}_{\mathrm{x}}(1.9 \mathrm{mg}$ coke/g cat 
h) and $\mathrm{Ru} / \mathrm{MgAlO}_{\mathrm{x}}(1.3 \mathrm{mg}$ coke/g cat $\mathrm{h})$ both had significantly lower carbon depositions when compared to Pt, Pd, Au, and Ir resulting in the highest catalytic actives (Tsyganok et al., 2003).

Noble metal catalysts offer the greatest stabilities across the board for DRM and other coke depositing processes. Their high expense prevents most commercialization at the industrial scale. Transition metal catalysts ( $\mathrm{Ni}, \mathrm{Fe}$, and $\mathrm{Co}$ ) attempt to fill the void as cost effective alternatives, but they face issues in providing effective coking resistance. When alone, many of these catalysts provide poor coking resistance, quickly deactivating as active sites are covered in carbon, however, when promoted on coking resistant supports with basic characteristics like alkali and alkaline earth metal oxides, carbon deposition can be greatly reduced. Supports with basic sites offer increased dissociative desorption rates of $\mathrm{CO}_{2}$ lending positive improvements in DRM with enhancements of $\mathrm{CO}_{2}$ conversion leading to the reduction in of coking. Basic sites contribute to the improvement of acidic $\mathrm{CO}_{2}$ chemisorption via a catalytic support, preventing carbon deposition, and providing catalytic stability. Supports such as $\mathrm{Al}_{2} \mathrm{O}_{3}$ and $\mathrm{MgO}$ have high basic characteristics as indicated via temperature programmed desorption (TPD) where the two exhibit desorption rates of $33.7 \mathrm{~mol}$ g-1 and 12.8 mol g-1, respectively (Aziz, Jalil, Wongsakulphasatch, \& Vo, 2020). Other popular supports $\left(\mathrm{ZrO}_{2}, \mathrm{TiO}_{2}, \mathrm{SiO}_{2}, \mathrm{CaO}\right.$, etc.) depict similar $\mathrm{CO}_{2}$ adsorption characteristics. The basicity of a catalyst surface depends upon numerous properties of metal, such as charge status, coordination number, size, and electronegativity of the oxidative state. This basicity is ultimately determined by surface oxygen sites, although the introduction of point defects in the lattice caused by high pretreatment temperatures of the support. $\mathrm{MgO}$ with more of point defects caused by vacancies of $\mathrm{Mg}^{2+}$ are found to have even higher basicity as they create three major types of basic sites in the lattice: edges, vertexes, and pits. High thermal treatments cause more of these lattice defects to occur which increases the density of basic sites causing greater reactivity to acidic $\mathrm{CO}_{2}$ 
(Aziz, Jalil, Wongsakulphasatch, \& Vo, 2020). High basicity supports only assist with half of the DRM reaction, typically being impregnated with other metals and/or metal oxides that have affinities for reacting with $\mathrm{CH}_{4}$. Although the addition of more material on the surface of basic supports would suggest the reduction of active basic sites, one study showed that the addition of $\mathrm{Ni}$ and Pt on the surface of $\mathrm{MgO}$ did not lead to such reductions (Singha, Shukla, Sandupatla, Deo, \& $\mathrm{Bal}, 2017) . \mathrm{Ni} / \mathrm{MgO}$ catalysts used in DRM experiments revealed higher catalytic intensity while maintaining stable for long time periods (Zhang, Li, Li, Zhang, \& Hu, 2013). After a 10hour DRM reaction at $650^{\circ} \mathrm{C}$, the two $\mathrm{Ni} / \mathrm{MgO}$ catalysts tested the commercial Mg sample only showed a $16 \%$ reduction in methane activation while the synthesized $\mathrm{Ni} / \mathrm{MgO}$ (111) catalyst virtually lost none of its initial catalytic activity for both methane and carbon dioxide, $\sim 62 \%$ and $\sim 71 \%$ respectively.

\subsubsection{DRM Catalyst-Microwave Irradiation}

DRM is heated via conventional methods between $700-950{ }^{\circ} \mathrm{C}$ and serves at the current industry standard, where the entirety of the reactionary environment is heated before reacting with the inlet gasses. Since the gas and catalysts are heated together, several reactions can take place both primarily on the surface of the catalyst but also secondarily in the heated gaseous environment that can result in undesirable products. Selective heating would provide excellent opportunities for solid-gas interactions regarding heterogenous catalysts and their gaseous environments as it has been reported to create incredibly large temperature differentials between surface of a catalyst and the gas that surrounds it. MW heating is the result of exposure of dielectric materials to a high frequency electromagnetic field. Gaseous components do not offer such dielectric properties alone and fail to interact with MW irradiation. Therefore, the utilization of solid-gas interactions on catalytic active sites and/or material supports that display these necessary dielectric properties 
develop temperature gradients $\left(\sim 300^{\circ} \mathrm{C}\right)$ between themselves and the gaseous environment. This high temperature gradient between the heated active sites and the cooler gas offers a primary reaction path on the surface of the particles that mitigate secondary gas phase reactions from occurring. Several studies regarding the addition of MW-absorbing metals, metal oxides, and carbon-based catalysts $\left(\mathrm{Fe}_{3} \mathrm{O}_{4}, \mathrm{MoO}_{3}, \mathrm{Ni}, \mathrm{Fe}, \mathrm{C}\right.$, etc. $)$ explored the potential of these catalyst to increase methane conversion, increase the selectivity of heavy hydrocarbons and aromatics, and reduce the thermal energy needed for coupling. The two preferred and highly studied DRM catalyst types are carbon based and metal based, although the latter has been researched more thoroughly. Metal-based catalysts are more popular with their high abundance and better understood reactionary pathways. Metallic iron and nickel under MW radiation have shown yields of methane conversion as high as $78.8 \%$ and $80.9 \%$, respectively (Wan, Chen, Lee, \& Depew, 2000). Compared to a popular methane coupling catalyst $\mathrm{Mo}_{2} \mathrm{C} / \mathrm{ZSM}-5$, carbonized from $\mathrm{MoO}_{3} / \mathrm{ZSM}-5$, heated using conventional methods, only $5.7 \%$ conversion was seen, although the selectivity for aromatics was noticeably higher even at this low conversion rate (Solymosi et al.). Ni is one of the most popular catalysts for DRM reactions, as it produces some of the highest methane conversion rates out of nearly all other catalysts within literature, but it also is highly prone to carbon deposition on its surface. By itself, Ni catalysts quickly succumb to coking, inevitably deactivating. Ni-based catalysts typically are coated on supports with high basicity to combat the issue of coking, as the basic support assists in the desorption of $\mathrm{CO}_{2}$ and subsequently the carbon monoxide disproportionation (reverse Boudouard reaction). $\mathrm{Ni} / \mathrm{Al}_{2} \mathrm{O}_{3}$ is the most used $\mathrm{Ni}$ based catalyst in DRM due to its high conversion, low coking, and low cost. However, $\mathrm{Ni} / \mathrm{Al}_{2} \mathrm{O}_{3}$ cannot be heated within a MW reactor and must be mechanically mixed with other materials $(\mathrm{SiC})$ to reach reaction temperatures, adding a step that other catalysts would not typically need. Even so, after 6 hours 
the average conversions of $\mathrm{CH}_{4}$ and $\mathrm{CO}_{2}$ were $90 \%$ at $800{ }^{\circ} \mathrm{C}$ with $\mathrm{Ni} / \mathrm{Al}_{2} \mathrm{O}_{3}$ and $\mathrm{SiC}$ mixed and while the conventional heated catalyst yielded $79 \%$ conversion of both $\mathrm{CH}_{4}$ and $\mathrm{CO}_{2}$ (Ignacio de Dios García et al., 2021). $\mathrm{Ni} / \mathrm{Al}_{2} \mathrm{O}_{3}$ showed above an $11 \%$ increase compared to conventional heating, even when mixed with $\mathrm{SiC}$ as a heating medium. Although $\mathrm{Ni} / \mathrm{Al}_{2} \mathrm{O}_{3}$ cannot heat via $\mathrm{MW}$ irradiation alone, another study combined $\mathrm{NiO} / \mathrm{Al}_{2} \mathrm{O}_{3}$ with a commonly used catalyst support, biochar, for MW irradiation DRM tests.

Carbon-based catalysts are frequently used for DRM via two methods: they are directly used as the catalyst, and/or they act as the support to load a metal component by the means of mechanical mixing or impregnation (Longzhi Li, 2018). Carbon-supported metal catalysts are better at reforming when compared to metal oxide supported catalysts, although they are not as economical (Longzhi Li, 2018). Combining the two common catalysts $\left(\mathrm{NiO} / \mathrm{Al}_{2} \mathrm{O}_{3}\right.$ supported on biochar), samples with 5-10 wt\% Ni produce a consistent $\mathrm{CH}_{4}$ and $\mathrm{CO}_{2}$ conversion from $\sim 91 \%$ to $~ 84 \%$ and $\sim 93 \%$ to $88 \%$, respectively over a 120 -minute reaction (Longzhi Li et al., 2017). Each $\mathrm{NiAl}_{2} \mathrm{O}_{4}$ despite the amount of $\mathrm{Ni}$ used, was able to remain relatively stable during the DRM reaction, although each slightly lost some of its catalytic capabilities. Unlike metal catalysts, this catalytic reduction was not entirely due to coking on the surface of $\mathrm{NiAl}_{2} \mathrm{O}_{4}$, but instead was due to carbon consumption from char reacting with $\mathrm{CO}_{2}$. Carbon loss is unavoidable when using carbon-based materials and it eventually can affect stability of the material. The stability of $\mathrm{CH}_{4}$ conversion is related to the activity of $\mathrm{Ni} /$ biochar, and it is dependent of the rate of carbon deposition and consumption. Two phenomenon of carbon deposition and consumption are present during DRM and effect the stability of $\mathrm{Ni} /$ biochar differently. Carbon deposition is mainly derived from methane decomposition, and it can block the active centers while carbon consumption during the reforming reaction consists of the elimination of deposited carbon and the carbon contained in 
biochar because the activities of both carbons are totally different (Longzhi Li et al., 2017). The removal of deposited carbon partially recovers the activity of $\mathrm{Ni} /$ biochar and the depletion of the contained carbon in biochar cut down the interaction between $\mathrm{Ni} /$ biochar and the reactant gasses (Longzhi Li et al., 2017). Although carbon deposition and consumption occur in parallel during dry reforming, the rate of carbon deposition and consumption is slightly disproportionate and eventually affect the stability of $\mathrm{Ni} /$ biochar. An example of carbon deposition vs consumption disproportionality is shown below in Figure 2.13 (B. Fidalgo et al., 2011).

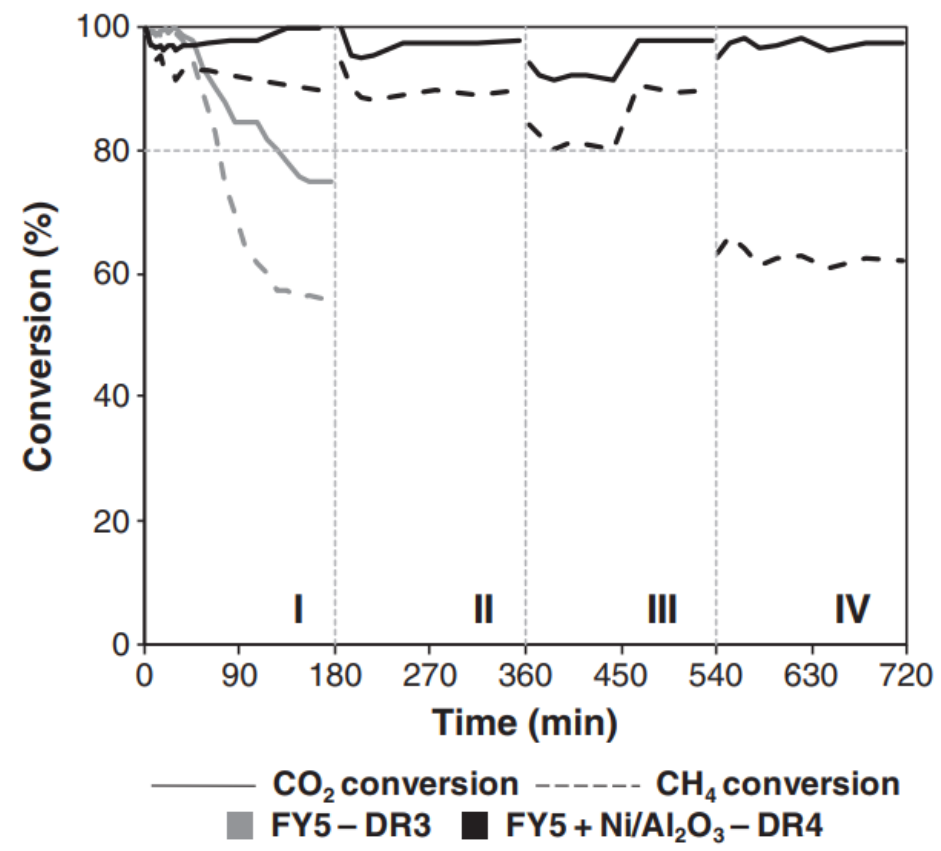

Figure 2.13: Comparison of the conversions of $\mathrm{CO}_{2}$ and $\mathrm{CH}_{4}$ obtained in the dry reforming experiments over the catalysts FY5 (experiment DR3) and FY5+Ni/ $\mathrm{Al}_{2} \mathrm{O}_{3}$ (experiment DR4) (B.

Fidalgo et al., 2011).

The microwave DRM (MWDRM) test was again conducted with $\mathrm{Ni} / \mathrm{Al}_{2} \mathrm{O}_{3}$ but instead impregnated on the surface of FY5, a biochar derived from coconut shells. The reaction rate of carbon dioxide to carbon monoxide is relatively stable for the entirety of the reaction with a conversion of $95-100 \%$, but the stability of the methane reaction rate slowly diminishes by $5-10 \%$ 
from the 360 - 540-minute mark. Carbon was being deposited on the surface of the active catalyst as well as slowly eroding the catalytic char, thus reducing the reaction rate on both fronts. After 540 minutes, the ratio of $\mathrm{CH}_{4}: \mathrm{CO}_{2}$ was altered from 1:1 to $3: 2$, where the reaction rate of $\mathrm{CH}_{4}$ quickly dropped to $60 \%$ conversion. The lack of $\mathrm{CO}_{2}$ in the inlet gas to prevent coking assisted in this large drop in conversion, but thereafter the reaction held quite steadily as less carbon could be removed from the bed.

\subsubsection{Current Catalyst Research}

Catalyst development for the conventional dry reforming of methane is abundant, but most papers cover only some form of Ni or platinum-based catalyst on different basic supports. Many of these conventional catalysts have proven excellent for their stability and good conversions of $\mathrm{CH}_{4}$ and $\mathrm{CO}_{2}$ but lack the ability to be heated with MW irradiation. Even though MW heating has been proven to be less costly, design friendly, and yield similar, if not better results than conventional DRM, there is very limited research on the topic and even more scarce application in industry. Research on common industry catalysts like Fe have very few studies regarding MWDRM reactions, and many of them are not focused directly on the benefit of adding a highly dielectric particle to a support and instead focus on biochar's that are rich in iron. Unlike Ni, iron oxides heat incredibly well within a MW reactor and have shown similar reaction rates to Ni based catalysts. $\mathrm{Fe}_{2} \mathrm{O}_{3}$ when mixed with cornstalk char at $10 \mathrm{wt} \%$ was able to convert $\mathrm{CH}_{4}$ and $\mathrm{CO}_{2}$ at an average of $90.8 \%$ and $95.2 \%$, respectfully for 160 minutes at $800^{\circ} \mathrm{C}$ without any significant deactivation (L. Li et al., 2019). A similar study used steel-making slag that contained $15 \%$ Fe by weight and mixed it with FY5 activated carbon, yielding stable conversions of $\mathrm{CH}_{4}$ and $\mathrm{CO}_{2}$ at $70 \%$ and $90 \%$, respectively at $800{ }^{\circ} \mathrm{C}$ (Jose M. Bermudez et al., 2012). Although the methane conversion was noticeably lower in the iron slag study, this was primarily due the slag 
itself being unable to fully heat within the MW reactor unlike other examples of Fe, and required a carbon based FY5 to be brought to temperature. Fe in the literature does not show significant coking when tested without the use of a high basicity catalyst, while $\mathrm{Ni}$ on the other hand is incredibly prone to coking in this case. Two studies tested $\mathrm{Fe} / \mathrm{SiC}$ in their $\mathrm{MW}$ reactors at $700{ }^{\circ} \mathrm{C}$ and $800{ }^{\circ} \mathrm{C}$, both again showing high and consistent reaction rates for $\mathrm{CH}_{4}$ and $\mathrm{CO}_{2}$ averaging $85 \%$ and $95 \%$ conversion, respectively, for the duration of their reaction times (F. Zhang et al., 2018). Fe based catalysts seem to have taken the back seat in the recent developments of MWDRM catalysts despite meeting all the criteria for an excellent catalyst. Fe is readily available, coke resistant, economically viable, and offers excellent activation to natural gasses. Fe has the same potential at optimizing DRM reactions like that of $\mathrm{Ni} / \mathrm{Al}_{2} \mathrm{O}_{3}$ and platinum-based metals but lacks much of the research it needs to be reliably used in industry. Additionally, LRC coal char assists MW heating and the DRM reaction as a catalyst, but little information is documented specifically on the use of coal char as a support. Using coal char was to further expand the understanding of its potential as MWDRM catalyst support. Continued research on Fe and its oxides may prove to bring research a step closer to sustainably creating synthesis gases to fuel ever increasing energy demands for the foreseeable future.

\subsection{Conclusions}

All industries aim to minimize costs and maximize profit margins through constant innovation and efficient optimization to each section of their processes to increase valuable product yield and decrease utility costs including raw materials, energy consumption, and waste removal. Invariably, one of the best solutions to help conquer these problems are when a process can reuse or repurpose an inapplicable byproduct to increase the yield of a desirable product. The reemergence of viable coal-derived fuel technologies and hydrocarbon fuel upgrading has sparked the need for further 
research to continue progressing towards alternative fuel options for the United States' growing energy demands. Currently, coal to fuel processes typically produces less marketable solid byproducts in the form of slag and char. These solid byproducts have limited applications and are usually hauled away in the form of waste as much of their stored chemical potential has long since been lost to previous reactions. However, coal char still contains trapped carbon within its microstructure the has the potential to be released in the form of profitable volatile gases used for fuel generation. Coal char is relatively stable even at high temperatures due to the microstructure being condensed and polymerized into a solid following a high temperature pyrolysis reaction, where much of the moisture and volatiles have already been removed from the original LRC. Although char is relatively unreactive, even at high conventionally desired temperatures, it possesses several properties that may lend itself to be further utilized as a heat syncing catalyst support or as fuel for continued syngas production. LRC when compared to HRC has many more impurities that are trapped within the solid matrixes that exit the pyrolysis reaction and can absorb MW radiation in the form of heat. This induced heating can assist in the bulk heating of catalyst beds or further be volatilized into gaseous fuels through the thermal differential seen between the carbon structure and MW absorbing impurities. If the solids within LRC char could be further driven off into the gaseous phase, the trapped carbons would need hydrogen or a hydrogen donating molecule since it is highly hydrogen deficient. The hydrogen source would allow for the carbon to be released in the form of volatilized hydrocarbons such as methane, ethylene, ethane, etc. This process is typically used when pyrolysizing hydrogen deficient coal and is known as hydropyrolysis where hydrogen reacts with the coal matrix to assist with reforming LRC. Methane is typically used for this as a hydrogen donating molecule since it is more available, contains more hydrogen per mole, and is cost effective. 
Alternatively, if char is unable to be reacted and is truly inert, it still has a great potential as a MW heat syncing catalyst support assisting with bulk bed heat distribution. Coal char is incredibly porous with a high surface area to weight ratio. Using coal char as a support could be incredibly advantageous. The porous microstructure of coal char can be infiltrated with catalytic particles, helping disperse them over its high surface area and heat these catalytic particles on the surface alone instead of needing to heat the entire reactor. Heating the char particles with MW irradiation creates a large temperature gradient between the surrounding atmosphere and its surface, promoting primary surface reactions and minimizing secondary gaseous reactions from occurring. A material or chemical requires dielectric properties to be heated with MW energy. MW heating of heterogeneous catalysts are highly beneficial to gaseous reactions since a gas alone cannot be heated with MW energy. An example fuel generation process where this is the case is seen with the dry reforming of methane, where methane and carbon dioxide are activated at high temperatures, typically over the surface of a catalyst, to produce hydrogen and carbon monoxide known as syngas. A DRM reaction requires high temperatures $\left(700{ }^{\circ} \mathrm{C}-950{ }^{\circ} \mathrm{C}\right)$ when heated conventionally even with a catalyst. Some studies have suggested that the addition of MW assisted heating could enhance the production of syngas in these reactions by providing a high heat differential for the adsorption reaction of the gases on the catalysts surface. The use of coal char as the MW induced heat syncing catalyst support has the potential to reduce the necessary energy required for the dry reforming of methane and it is one of many topics that is covered in this dissertation along with several other under-researched topics.

\subsection{Purpose}

This literature review found that several topics that have yet to be fully covered and expanded: (1) dried PRB coal char use as a catalyst for upgrading methane and carbon dioxide, (2) detailed study 
of the solid-gas reactions between dried PRB coal char, metal oxide catalysts, methane and carbon dioxide, (3) exploration of the effects of MW radiation on dried PRB coal char, coal charmethane/carbon dioxide interactions, metal oxide catalysts, shaped metal oxide catalysts and char/ $\mathrm{M}_{\mathrm{n}} \mathrm{O}_{\mathrm{x}}$ catalysts, (4) the combination of hydropyrolysis and DRM process principles for the further utilization of dried PRB coal char, (5) Exploration of the potential of reacting methane and carbon dioxide with coal char-catalysts via MW induction heating compared to conventional heating methods under similar conditions, (6) Analyzation of the products yielded from any solidgas interactions with these catalysts and contrast them with traditional heating methods. This study and review explore the possibilities of utilizing dried coal char and metal oxide catalysts for upgrading methane and carbon dioxide into energy dense alternative fuels that are imperative towards meeting the United States' future energy requirements and further improving the use of coal-derived fuel production. 


\section{Chapter 3: Synthesis and Dielectric Properties of Shaped Metal Oxides Suitable for the MW-Induced Dry Reforming of Methane}

\subsection{Introduction}

The foundation of modern catalyst theory is based on the Sabatier Principle which states, the surface-adsorbates interaction must be an optimal amount: not too weak to be inert toward the reactants and not too strong to poison the surface and avoid desorption of the products (Anders L. et al., 2011). The latter half of Sabatier's principle holds significant weight in catalyst research, as surface-adsorbates that chemisorb too strongly will inevitably poison any catalyst. Catalyst poisons are any substance that decrease the reaction rate by binding to active sites (B. Calvin, 2001). Methane reformation processes are particularly prone to catalyst deactivation, as poisoning occurs when methane is dehydrogenated. The hydrogen desorbs for the catalyst surface leaving solid carbon deposited along the material surface. This carbon depositing, also known as coking, is a primary cause for concern regarding any form of methane reformation. Methane reforming catalyst research is often to discover materials that demonstrate exceptional methane conversion while minimizing coking on its surface. Platinum group metals present an excellent example of an ideal dry reforming of methane (DRM) catalyst, since they demonstrate high conversion rates as well as high resistivity to coking deactivation (Liu, D et al., 2010). Unfortunately, catalysts of this variety are seldom used for methane reforming since they are all incredibly expensive, leaving the door wide open for implementation of other cost-effective catalysts.

Much of methane reforming catalyst research primarily focuses on the usage of transition metalbased materials, mainly regarding $\mathrm{Ni}, \mathrm{Fe}$, and Co. Alone, each of these metals are not suitable for DRM. Each are severely susceptible to coking and quickly deactivated. However, when these 
transition metals, namely $\mathrm{Ni}$, are impregnated on high basicity supporting materials, carbon deposition can be greatly reduced. Supports with basic sites offer increased dissociative desorption rates of $\mathrm{CO}_{2}$ lending positive improvements in DRM with enhancements of $\mathrm{CO}_{2}$ conversion leading to the reduction in of coking. Basic sites contribute to the improvement of acidic $\mathrm{CO}_{2}$ chemisorption via a catalytic support to prevent carbon deposition. Improved $\mathrm{CO}_{2}$ adsorption offers reactionary pathways for deposited carbon to react and desorb in the form of CO (Aziz, M. A., Jalil, A. A., Wongsakulphasatch, S., Vo, D. N., 2020). Effectively, this reaction removes much of the coking seen from the methane reaction on the catalyst surface providing catalytic stability. High basicity supports mainly assist with the $\mathrm{CO}_{2}$ portion of the DRM reaction, typically being impregnated with other metals and/or metal oxides that have affinities for reacting with $\mathrm{CH}_{4}$. $\mathrm{Ni} / \mathrm{MgO}$ and $\mathrm{Ni} / \mathrm{Al}_{2} \mathrm{O}_{3}$ are common transition metal/basic supported catalysts used in DRM experiments revealed higher catalytic intensity while maintaining stable for long time periods (Zhang, Li, Li, Zhang, \& $\mathrm{Hu}, 2013$ ). After a 10 -hour DRM reaction at $650{ }^{\circ} \mathrm{C}$, the two $\mathrm{Ni} / \mathrm{MgO}$ catalysts tested the commercial $\mathrm{Mg}$ sample only showed a $16 \%$ reduction in methane activation while the synthesized $\mathrm{Ni} / \mathrm{MgO}$ (111) catalyst virtually lost none of its initial catalytic activity for both methane and carbon dioxide, $\sim 62 \%$ and $\sim 71 \%$ respectively (Zhang, Li, Li, Zhang, \& $\mathrm{Hu}$, 2013). Although supports such as $\mathrm{Al}_{2} \mathrm{O}_{3}$ and $\mathrm{MgO}$ are commonly used as catalyst supports, other supporting materials, namely alkaline earth metal oxides $(\mathrm{CaO}, \mathrm{BrO}$, and $\mathrm{SrO})$, depict similar $\mathrm{CO}_{2}$ adsorption characteristics (Aziz, Jalil, Wongsakulphasatch, \& Vo, 2020; Buevskaya et al., 1987). These materials are low in cost, readily availability, and are affordably recoverable while maintaining effective catalytic properties. Alkaline earth metal oxides are particularly favorable for OCM and DRM reactions. They can form Lewis base pairs with acidic gases like that of $\mathrm{CO}_{2}$. Alkaline earth metal oxides have relatively low electro negativities, are readily available, are 
economically viable, and have readily been theorized and tested to show their capability of making these Lewis base-pairs with methane and carbon dioxide (Aika, K., \& Nishiyama, T., 1989). Alone, these are not the end-all for methane reforming since these are more so related to the reforming of $\mathrm{CO}_{2}$ present in DRM reaction, although some studies due note alkaline earth metal oxides do have some reforming properties (Buevskaya, O. et al., 1987; Prince, B. et al., 2013). Each of these can provide necessary basic sites needed for DRM reactions and may work well in the presence of a well suited, methane activating material. For instance, molybdate $\left(\mathrm{MoO}_{3}\right)$ catalysts are known for their ability to upgrade methane into highly profitable aromatics, namely benzene and naphthalene when decorated within a porous zeolite structure. Molybdate molecules attach themselves to the Bronsted acid sites located within the zeolite's pores, although the $\mathrm{MoO}_{3}$ is not what initiates the MDA process Mo/HZSM-5 catalysts have an induction period prior to the formation of aromatics. Methane partially carburizes the Mo into molybdenum oxycarbide ( $\mathrm{MoO}_{\mathrm{x}} \mathrm{C}_{\mathrm{y}}$ ), releasing $\mathrm{CO}, \mathrm{CO}_{2}$, and $\mathrm{H}_{2} \mathrm{O}$ as products (Liu, Wang, Ohnishi, \& Ichikawa, 1999). The $\mathrm{MoO}_{\mathrm{x}} \mathrm{C}_{\mathrm{y}}$ species can convert $\mathrm{CH}_{4}$ into $\mathrm{C}_{2+}$ products, but as the oxycarbide species is fully carburized into molybdenum carbide $\left(\mathrm{MoC}_{\mathrm{x}}\right)$, benzene formation can finally occur withing the zeolite pores (Lezcano-González et al., 2016). Although HZSM-5 zeolites are not used as the support for this study, molybdate catalysts have also been suggested to be involved with the creation/production of ethylene during the intermediate reaction. Carbidic molybdenum hydrogenated to form molybdenum carbene and methylene bridge (in the case of dimeric species) (Xing et al., 2010; Zhou et al., 2012). The $\mathrm{Mo}=\mathrm{CH}_{2}$ represents a Lewis acid-base pair, polarizing and activate methane to form a negatively charged methyl group bound to the Mo cation and a positively charged methyl group is formed by reaction of the carbene with the resulting proton from the $\mathrm{C}-\mathrm{H}$ bond scission. The two $\mathrm{CH}_{3}$ groups then undergo $\mathrm{C}-\mathrm{C}$ coupling, desorbing after the 
activation of a third methane molecule to replace the original carbene ligand incorporated in the formed ethylene. The combining of these well-known capabilities of molybdenum methane coupling particles with the $\mathrm{CO}_{2}$ adsorption characteristics of alkaline earth metals could be incredibly advantageous in the development of new, MWDRM catalysts.

Unfortunately, when it comes to catalyst behavior within MW reactor environments, popular catalysts such as $\mathrm{Ni} / \mathrm{MgO}$ and $\mathrm{Mo}$ begin to present problems. Both, on their own, are poor at absorbing electromagnetic radiation and dispersing that energy as heat. This alone is the key eliminating factor for several typical DRM catalysts in their current form from being viable as MWDRM catalyst testing and this study. However, the concept of implanting metal particles on high basicity supports provides the necessary scaffolding to develop new and/or reformulate older catalysts for future reactor designs implementing MW heating over conventional reactors. MW reactor development has gained major support as a cost effective, efficient, bulk bed heating solution. MW heating is immediate and is highly efficient at converting energy into bulk heating depending on the material being irradiated (Metaxas, A. C., \& Meredith, R. J., 1988). Heating is procured simply via energy transfer of MW radiation into a material and the dissipation of that energy as heat. Conventional heating processes use convection, conduction, and radiation phenomena thermal gradients to transfer energy to a material's surface. MW energy, however, delivers energy directly to materials through molecular interactions (loss mechanisms) with electromagnetic field via conversion of electromagnetic energy into thermal energy. Loss mechanisms cause a high rate of heating and a high efficiency of energy conversion are expected in a short time. The key to sufficient MW heating is based on the ability of a material to store and dissipate electromagnetic energy. The ability of a substance to convert electromagnetic energy into heat at a given frequency and temperature is determined by the following equation: $\tan \delta=\varepsilon " / \varepsilon$. 
Tangent delta $(\delta)$, or loss tangent, is the dissipation factor of a sample or how efficiently MW energy is converted into thermal energy. It is defined as the ratio of the loss factor ( $(")$, to the dielectric constant $\left(\varepsilon^{\prime}\right)$. Dielectric loss is the amount of input MW energy that is lost to a sample when dissipated as heat (Barba \& d'Amore, 2012). Dielectric properties of material are crucial parameters in MW heating; they express the energy coupling of a material with electromagnetic MW field and, thus, the heating feasibility (Metaxas \& Meredith, 1983; Schubert \& Regier 1995; Tang et al., 2002). Dielectric properties can be affected by several factors including MW frequency, temperature, and chemical composition of the materials (Kraszewski, 1996; Tang et al., 2002). Additionally, dielectric properties of solid particles may also be changed with different phase transitions of the material. It is commonly seen within literature that materials created with new phase morphologies also exhibit new dielectric properties that are affected differently within a MW reactor environment. Synthesizing and testing new morphologies of typical catalysts with MW radiation would be highly advantageous for the chemical manufacturing industry. Customary catalysts could be improved upon by simply changing the morphology of the material without changing the material itself or other process parameters to optimize a reaction system. As the popularity and technology of MW reactors continues to advance, capable, cost-effective catalysts will be in high demand.

Fe and its oxides do not have this issue, as it heats well within a MW reactor, and has shown similar reaction rates to $\mathrm{Ni}$ based catalysts. Fe has shown promising catalytic methane reforming results in recent years as an acceptable alternative to $\mathrm{Ni}$ or platinum group-based catalysts while remaining affordable and in abundance. Several studies have reported positive Fe/char-based catalyst DMR reactions, one showing conversions of $\mathrm{CH}_{4}$ and $\mathrm{CO}_{2}$ at an average of $90.8 \%$ and $95.2 \%$, respectively and another using steel-making slag that contained $15 \%$ Fe by weight and 
mixed it with FY5 activated carbon, yielding stable conversions of $\mathrm{CH}_{4}$ and $\mathrm{CO}_{2}$ at $70 \%$ and $90 \%$, respectively at $800{ }^{\circ} \mathrm{C}$ (L. Li et al., 2019; Jose M. Bermudez et al., 2012). Additionally, Fe was not reported to be prone to coking when tested without the use of a high basicity catalyst. Two studies tested $\mathrm{Fe} / \mathrm{SiC}$ in their MW reactors at $700{ }^{\circ} \mathrm{C}$ and $800{ }^{\circ} \mathrm{C}$, both again showing high and consistent reaction rates for $\mathrm{CH}_{4}$ and $\mathrm{CO}_{2}$ averaging $85 \%$ and $95 \%$ conversion, respectively, for the duration of their reaction times (F. Zhang et al., 2018). Iron-based catalysts have limited research in the field of DRM reactions. Regarding currently reported studies, the utilization of Fe and its oxides for the MWDRM process is imperative. Iron is readily available, coke resistant, dielectrically favorable, economically viable, and offers excellent activation of $\mathrm{CH}_{4}$ and $\mathrm{CO}_{2}$, thus requiring significant attention for the future of MWDRM catalyst design.

The purpose of this study is to consolidate gaps within previous understandings of DRM catalyst designs and new MW reactor catalyst requirements by synthesizing a variety of new, MW inducible materials into different morphologies and testing these for any changes seen in their dielectric and $\mathrm{CH}_{4} / \mathrm{CO}_{2}$ reformation capabilities. The goal of this study is to develop shaped particles with high loss factors and efficient MWDRM catalytic properties for upgrading $\mathrm{CH}_{4}$ and $\mathrm{CO}_{2}$ into higher valued fuels. The approach for developing an ideal MWDRM catalyst was by experimenting with varied synthesis parameters to control the morphology and size of the particles and determine their dielectric characteristics. Materials were chosen based on their previous success as methane and/or carbon dioxide upgrading catalysts. Altering catalyst structures to have added edges, voids, and corners is advantageous as these features typically provide additional active sites for reactions to occur. The nature MW induction on a particle exploits certain microstructures that feature thin protrusion or are thin by design since this heating approach begins in the center of the particle, radiating from the inside to the outside. Therefore, thin structures have 
the advantage of heating rapidly and acquiring exceedingly high surface temperatures without the need for the local temperature of the reactor to meet that same threshold. Thinly designed catalysts conjointly have high surface areas and greater chances of developing active sites compared to simple cubic and spherical particles. Coupling these features with materials that possess exceptional dielectric properties, such as high loss factors, lend to the theory that they would also serve as excellent catalytic particles in MW reactor environments. Atypically shaped catalytic particles were synthesized by manipulations to synthesis parameters, namely changing surfactant concentrations, reaction temperatures, autoclave treatment times, and precursor concentrations. Alterations in the size and morphology were performed to observe if any changes in dielectric behaviors of the materials compared to typical stock nanoparticles. Additionally, testing of these material's catalytic properties both with and without Powder River Basin (PRB) coal char for changes in $\mathrm{CH}_{4} / \mathrm{CO}_{2} \mathrm{MW}$ reforming is reported in the following chapter. Low-rank coal chars, such as PRB, possess impurities that are dielectrically favorable, heating well within MW/electromagnetic environments, severing as a bulk heating medium for catalyst beds. Morphology and size alterations combined with MW induction are supported to accelerate reaction rates on catalyst active sites when contacting reactant gases. These particles were evaluated using scanning electron microscopy (SEM), X-ray diffraction (XRD), and dielectric property analysis. The chemistry behind catalyst synthesis is not necessarily complex at face value. The reproducibility of catalyst synthesis is one of the most basic problems. Most processes used in creation of the following compounds were completed via reduction, coprecipitation, and/or impregnation with some processes needing further treatments such as calcination, sintering, or hydrothermal treatment. All significant preparation parameters were systematically analyzed and 
recorded to maintain repeatability and record how changes in process parameters changed the final product.

\subsection{Experimental Procedures: Shaped Catalyst Synthesis}

\subsection{1 $\mathrm{Fe}_{3} \mathrm{O}_{4}$ Nanorods}

A typical solution would start with a mixture of $1 \mathrm{mmol}$ of iron (II) sulfate heptahydrate $\left(\mathrm{FeSO}_{4}\right.$ - $\left.7 \mathrm{H}_{2} \mathrm{O}\right)$ and $2 \mathrm{mmol}$ of iron (III) chloride hexahydrate $\left(\mathrm{FeCl}_{3} \cdot 6 \mathrm{H}_{2} \mathrm{O}\right)$ were dissolved in a $100 \mathrm{ml}$ beaker with $40 \mathrm{ml}$ of deoxygenated DI water under magnetic stirring at $300 \mathrm{rpm}$ for 5 minutes. Then the solution was transferred slowly into a Teflon lined autoclave. Next, $8 \mathrm{ml}$ of benzene was laid on the surface of the iron solution to block oxygen from entering the reaction. $5 \mathrm{ml}$ of a $4 \mathrm{M}$ ethylene diamine solution was loaded into a syringe that was connected to a plastic tube held in place under the benzene layer. The ethylene diamine was then injected into the iron solution at a rate of $0.5 \mathrm{ml} / \mathrm{min}$ without stirring as a dark black precipitate formed. The Teflon lining was placed in a stainless-steel autoclave and treated at $120{ }^{\circ} \mathrm{C}$ for 20 hours and allowed to cool to room temperature. The precipitate was collected via magnetic separation and washed with DI and EtOH three times each. The washed precipitate was dried at $80{ }^{\circ} \mathrm{C}$ in open air for 12 hours. Particle morphology and sizes were manipulated by changing ethylenediamine concentrations, the ratio of $\mathrm{Fe}$ (III) to $\mathrm{Fe}$ (II) and stirring rates.

\subsection{2 $\mathrm{Fe}_{3} \mathrm{O}_{4}$ Nanoflakes}

Two solutions were prepared for the formation of $\mathrm{Fe}_{3} \mathrm{O}_{4}$ nano-flakes, labeled solution $\mathrm{A}$ and solution B. Solution A used a mixture of $1.39 \mathrm{~g}$ of iron (II) sulfate heptahydrate and $0.209 \mathrm{~g}$ of sodium acetate $(\mathrm{NaAc})$ were stirred in $75 \mathrm{ml}$ of DI water for 30 minutes or until fully dissolved in open air. Solution B was a $25 \mathrm{ml}$ solution of $2.4 \mathrm{M}$ sodium hydroxide as the reducing agent. 
Solution A was placed in a 3-necked, round bottom flask with a magnetic stir bar and submerged in a heated silicon oil bath. The oil was heated until the solution in the flask was held stable at 80 ${ }^{\circ} \mathrm{C}$. Solution B was placed in a syringe and connected to the round bottom flask after 30 minutes of stirring solution A at $80{ }^{\circ} \mathrm{C}$ for 30 minutes. Solution B was injected into solution A at a rate of $0.5 \mathrm{ml} / \mathrm{min}$ as the solution changed from orange, to light green, to dark orange/red, to dark green, to grey and then black as the $\mathrm{pH}$ increased $>9.0$ with the addition of $\mathrm{NaOH}$. The combined solution then stirred for 6 hours at $80^{\circ} \mathrm{C}$. Afterwards, the beaker was removed from the oil bath and allowed to cool to room temperature. The precipitate was separated via centrifuge and magnetic separation as it was washed with DI and $\mathrm{EtOH}$ three times each, respectively. The precipitate was then dried at $80{ }^{\circ} \mathrm{C}$ in atmospheric conditions. Particle morphology and sizes were manipulated by changing NaAc concentrations, the stirring rate, and reaction time. Shorter reaction times, such as 3 hours, would lead to flake particles with voids and the emergence of cubic shaped particles.

\subsection{3 $\mathrm{Fe}_{3} \mathrm{O}_{4}$ Micro Platelets and Octahedrons}

Initial preparation of $\alpha-\mathrm{Fe}_{2} \mathrm{O}_{3}$ platelets

A solution of $0.6 \mathrm{~g}$ of iron (III) acetylacetonate $\left(\mathrm{Fe}(\mathrm{acac})_{3}\right)$ was dispersed in $50 \mathrm{ml}$ of triethylene glycol (TEG) and stirred at $600 \mathrm{rpm}$ for 30 minutes. A $20 \mathrm{ml}$ second solution of $6 \mathrm{M} \mathrm{NaOH}$ was loaded in a syringe and injected into the iron solution at a rate of $0.5 \mathrm{ml} / \mathrm{min}$. Afterwards the total solution was allowed to stir for 1 hour. The solution was then placed in a Teflon lined autoclave for 5 hours at $190{ }^{\circ} \mathrm{C}$. The $\alpha-\mathrm{Fe}_{2} \mathrm{O}_{3}$ precipitate was then centrifuged and washed with $\mathrm{EtOH}$ and DI three times each, respectively. Then the $\alpha-\mathrm{Fe}_{2} \mathrm{O}_{3}$ was allowed to dry at $80{ }^{\circ} \mathrm{C}$ in open air.

\section{Initial preparation of $\alpha-\mathrm{Fe}_{2} \mathrm{O}_{3}$ octahedrons}


A solution of $0.6 \mathrm{~g}$ of iron (III) acetylacetonate $\left(\mathrm{Fe}(\mathrm{acac})_{3}\right)$ was dispersed in $100 \mathrm{ml}$ of triethylene glycol (TEG) and stirred at $600 \mathrm{rpm}$ for 30 minutes. A $20 \mathrm{ml}$ second solution of $6 \mathrm{M} \mathrm{NaOH}$ was loaded in a syringe and injected into the iron solution at a rate of $0.5 \mathrm{ml} / \mathrm{min}$. Afterwards the total solution was allowed to stir for 1 hour. The solution was then placed in a Teflon lined autoclave for 5 hours at $190{ }^{\circ} \mathrm{C}$. The $\alpha-\mathrm{Fe}_{2} \mathrm{O}_{3}$ precipitate was then centrifuged and washed with $\mathrm{EtOH}$ and DI three times each, respectively. Then the $\alpha-\mathrm{Fe}_{2} \mathrm{O}_{3}$ was allowed to dry at $80{ }^{\circ} \mathrm{C}$ in open air.

\subsubsection{Reduction of $\alpha-\mathrm{Fe}_{2} \mathrm{O}_{3}$ to $\mathrm{Fe}_{3} \mathrm{O}_{4}$ platelets and octahedral particles}

The yield of $\alpha-\mathrm{Fe}_{2} \mathrm{O}_{3}$ platelets were mixed into a solution of $0.477 \mathrm{~g}$ of sodium borohydride $\left(\mathrm{NaBH}_{4}\right)$ and $38 \mathrm{ml}$ of DI water at $300 \mathrm{rpm}$ for 10 minutes. The solution was then added to a 50 $\mathrm{ml}$ Teflon lined autoclave and hydrothermally processed for 12 hours at $160{ }^{\circ} \mathrm{C}$. Then black precipitate was washed three times with EtOH and magnetically separated. The precipitate was then dried at $80{ }^{\circ} \mathrm{C}$ for 6 hours or until dry.

\subsection{4 $\mathrm{CaMoO}_{4}$ Micro-Needles/Platelet Clusters}

A $75 \mathrm{ml}$ solution of $\mathrm{N}, \mathrm{N}$-dimethylacetamide (DMAc) and DI water was produced at a ratio of 14.3:85.7 and stirred at $600 \mathrm{rpm}$ for 10 minutes. Then $0.222 \mathrm{~g}$ of calcium chloride $\left(\mathrm{CaCl}_{2}\right)$ was added to the $75 \mathrm{ml}$ solution and placed in a sonication bath for 30 minutes until dissolved. The solution was placed back on a stir plate and $2.472 \mathrm{~g}$ of ammonium molybdate $\left(\left(\mathrm{NH}_{4}\right)_{6} \mathrm{Mo}_{7} \mathrm{O}_{24}\right)$ was added and stirred at $600 \mathrm{rpm}$ until dissolved. Stirring was continued for 15-20 minutes until a fluffy white precipitate was suddenly crashed out of solution. The solution with the precipitate was then transferred to a $100 \mathrm{ml}$ Teflon-lined autoclave and hydrothermally treated at $180^{\circ} \mathrm{C}$ for 30 minutes before being cooled to room temperature. The precipitate was centrifuged and washed with EtOH three times. The precipitate was placed on a hotplate set at $80{ }^{\circ} \mathrm{C}$ for 6 hours to dry in open air. 
Alterations to the morphology of $\mathrm{CaMoO}_{4}$ were prepared via altering the ratio of DMAc to water, altering the amount of $\mathrm{CaCl}_{2}$ used, and altering the hydrothermal treatment temperatures.

\subsection{5 $\mathrm{CaMoO}_{4}$ Platelets}

A $75 \mathrm{ml}$ solution of $\mathrm{N}$, N-dimethylacetamide (DMAc) and DI water was produced at a ratio of 1:4 and stirred at $600 \mathrm{rpm}$ for 10 minutes. Then $0.333 \mathrm{~g}$ of calcium chloride was added to the $75 \mathrm{ml}$ solution and placed in a sonication bath for 30 minutes until dissolved. The solution was placed back on a stir plate and $2.472 \mathrm{~g}$ of ammonium molybdate $\left(\left(\mathrm{NH}_{4}\right)_{6} \mathrm{Mo}_{7} \mathrm{O}_{24}\right)$ was added and stirred at $600 \mathrm{rpm}$ until dissolved. Stirring was continued for 15-20 minutes until a fluffy white precipitate was suddenly crashed out of solution. The solution with the precipitate was then transferred to a $100 \mathrm{ml}$ Teflon-lined autoclave and hydrothermally treated at $180{ }^{\circ} \mathrm{C}$ for 30 minutes before being cooled to room temperature. The precipitate was centrifuged and washed with EtOH three times. The precipitate was placed on a hotplate set at $80{ }^{\circ} \mathrm{C}$ for 6 hours to dry in open air.

\subsection{6 $\mathrm{SrMoO}_{4}$ Faceted Micro-Spheres}

A beaker was filled with $40 \mathrm{ml}$ of DI water and adjusted to $\sim 6 \mathrm{pH}$ by added very dilute nitric acid dropwise (Solution A). Next, a second solution (Solution B) of $1.48 \mathrm{~g}$ of strontium nitrate $\left(\mathrm{Sr}\left(\mathrm{NO}_{3}\right)_{2}\right)$ was magnetically stirred into $20 \mathrm{ml}$ of DI water until dissolved. A third solution of 20 $\mathrm{ml}$ of DI water and $1.23 \mathrm{~g}$ of ammonium molybdate $\left(\left(\mathrm{NH}_{4}\right)_{6} \mathrm{Mo}_{7} \mathrm{O}_{24}\right)$ was stirred until dissolved (Solution C). Solutions B and C were then combined and stirred for an additional 15 minutes. This solution was then slowly added dropwise to Solution A and stirred at $600 \mathrm{rpm}$ for 30 minutes. Dilute ammonium $\left(\mathrm{NH}_{4} \mathrm{OH}\right)$ was added to adjust the $\mathrm{pH}$ of the solution to 8 . The final solution was added to a $100 \mathrm{ml}$ Teflon-lined autoclave and hydrothermally treated at $80^{\circ} \mathrm{C}$ for 8 hours. The $\mathrm{SrMoO}_{4}$ precipitate was centrifuged and washed three times with $\mathrm{EtOH}$. The precipitate was heated at $80^{\circ} \mathrm{C}$ for 6 hours, then placed under vacuum to dry for an additional 6 hours. 


\subsubsection{Particle Characterizations}

The surface morphology of the samples was imaged using a field scanning electron microscope (Hitachi S-4700 Scanning Electron Microscope) operating at $45 \mathrm{eV}$ by placing a drop of the suspension solution carefully onto a glass slide on an aluminum stage and letting isopropyl alcohol evaporate slowly in air. Thereafter, each sample was coated with gold (Au) with a Denton Sputter Coater using an Au sputter technique for 140 seconds.

The X-ray diffraction (XRD) patterns for each sample was collected using a PANalytical X'Pert Pro X-ray Diffractometer CuK $\alpha$ radiation X-ray diffractometer over the range of $20^{\circ} \leq 2 \theta \leq 80^{\circ}$ with $40 \mathrm{~mA}$ and $45 \mathrm{kV}, 0.5 \mathrm{~s}$ step time, and $0.0216^{\circ}$ step size.

Dielectric testing was completed using the transmission line and free space method on an Agilent Technologies N5231A PNA-L Microwave Network Analyzer over a range of 0.1 to $13.5 \mathrm{GHz}$. The particles were added to a paraffin plug between 5-10 vol\%. Each dielectric data set was fitted to a polynomial and averaged to determine the true dielectric constant. The theoretical Looyenga Mixing Equation was then used to determine the true dielectric constant of each material.

\subsection{Results and Discussion}

\subsubsection{Synthesis of Iron Oxide $\left(\mathrm{Fe}_{3} \mathrm{O}_{4}\right)$}

The morphology and size of the nanoparticles were able to be selectively controlled by altering one or more of several growth variables. Altering the iron reagents and the concentrations of SDAs had the largest effect on the morphological outcome of the iron particles. Temperature conditions and reactor time also were crucial in manipulating the size of the final particles. However, facilitating the growth of shaped, single crystalline $\mathrm{Fe}_{3} \mathrm{O}_{4}$ nanoparticles was difficult for each experiment type, and took numerous alterations to obtain the desired morphologies and/or sizes. 
$\mathrm{Fe}_{3} \mathrm{O}_{4}$ nanocrystals typically remained in simple, naturally occurring morphologies such as cubic and/or spherical structures. $\mathrm{Fe}_{3} \mathrm{O}_{4}$ itself exists in a very niche region of the Pourbaix diagram, only forming under slightly electronegative conditions above a pH of 9 seen in Figure 3.1.

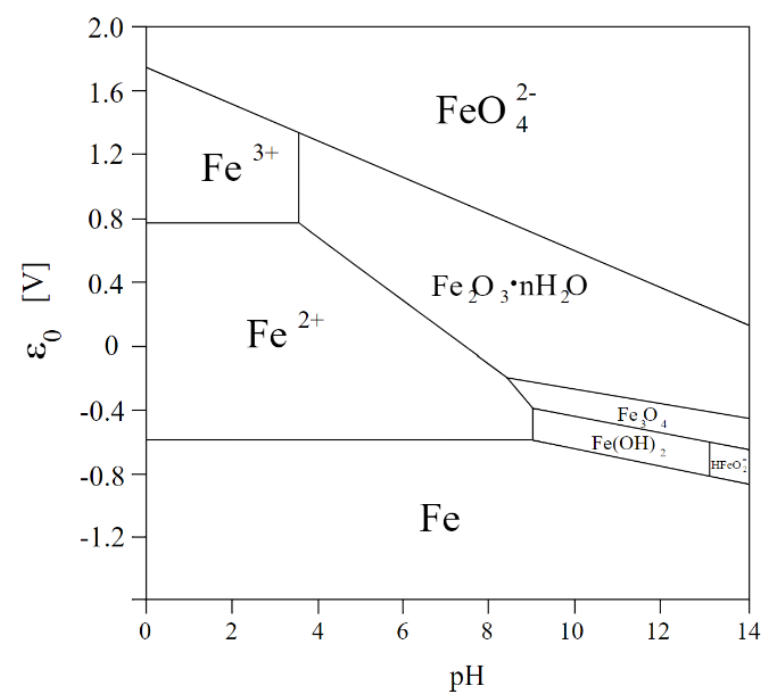

Figure 3.1: Pourbaix Diagram for Fe

\subsubsection{Changing the Morphology of $\mathrm{Fe}_{3} \mathrm{O}_{4}$}

Most experiments used a structure-directing agent (SDA) to facilitate atypical growth mechanisms of iron oxide nanocrystals during hydrothermal treatments. Surface-directing agents go by many names like capping agent, protective agent, surfactants, micro-emulsions, soft templates, etc., but for simplicity they are referred to as SDAs for this discussion. Most procedures fail to successfully manipulate the morphology or were incredibly inconsistent from experiment to experiment. Several tests using polyethylene glycol or hydrazine as SDA's did not successfully alter the morphology of $\mathrm{Fe}_{3} \mathrm{O}_{4}$ no matter what parameter was changed in the system. However, in nearly every experiment the bulk of the precipitate leaving the reaction was $\mathrm{Fe}_{3} \mathrm{O}_{4}$ with some traces of residual gothite $\left(\mathrm{Fe}(\mathrm{OH})_{2}\right)$. Goethite formation was expected since the formation of it is very close to that of $\mathrm{Fe}_{3} \mathrm{O}_{4}$ and goethite is an intermediate hydroxide molecule from the reduction process. The formation of $\mathrm{Fe}_{3} \mathrm{O}_{4}$ was incredibly consistent, but alterations to the morphology or size 
inconsistent for many reactions. This could be attributed to one of two mechanisms happening within the iron/surfactant solution, either: the reduction is occurring too quickly, or the SDA was not strong enough to overcome the natural crystal growth. The reduction of the acidic iron solutions creates a myriad of intermediate products before surpassing a pH of 9 to form $\mathrm{Fe}_{3} \mathrm{O}_{4}$. Many of these intermediates are varied concentrations of numerous iron hydroxide molecules (goethite being the most plentiful) and other iron oxide precipitates, namely $\alpha-\mathrm{Fe}_{2} \mathrm{O}_{3}$ and $\beta-\mathrm{Fe}_{2} \mathrm{O}_{3}$ that give the solution a reddish/orange color. The initial formation of $\alpha-\mathrm{Fe}_{2} \mathrm{O}_{3}$ and $\beta-\mathrm{Fe}_{2} \mathrm{O}_{3}$ are incredibly important since they are much easier to manipulate than $\mathrm{Fe}_{3} \mathrm{O}_{4}$. As the solutions basicity is increased, $\mathrm{Fe}_{2} \mathrm{O}_{3}$ molecules begin to nucleate out of the solution and interact the SDA in the surrounding environment as they grow into the desired configuration. After, the $\mathrm{Fe}_{2} \mathrm{O}_{3}$ precipitate continues to grow along the SDA, the initial shape of the final particle has been formed, creating its own hard template to continue growing. When the solution $\mathrm{pH}$ is greater than 9, the $\mathrm{Fe}_{2} \mathrm{O}_{3}$ structures are reduced into $\mathrm{Fe}_{3} \mathrm{O}_{4}$ while maintaining the initial morphology. Thereafter, hydrothermally treating these particles for several hours allows the remainder of the iron in solution to continue growing off the original structure. This approach to altering the morphology of $\mathrm{Fe}_{3} \mathrm{O}_{4}$ is consistent across most literature where an acidic solution containing an iron precursor and an SDA is reduced with a base to produce $\mathrm{Fe}_{3} \mathrm{O}_{4}$ via coprecipitation. However, some articles that aim to create larger, micro-sized particles employ a two-step hydrothermal process where they first partially reduce an iron solution to grow shaped $\alpha-\mathrm{Fe}_{2} \mathrm{O}_{3}$ before a second reduction creates the $\mathrm{Fe}_{3} \mathrm{O}_{4}$ (Zhao et al. 2018). The reduction of the solution must be done slowly to give the SDA time to facilitate the desired growth of a particle as they begin to nucleate as the $\mathrm{pH}$ is increased. A reduction completed too quickly resulted in numerous, small particles that crashed out of the 
suspension without interactions with the SDA as seen in Figure 3.2, where the addition of ethylenediamine was immediate, having no effect on nucleation of $\mathrm{Fe}_{3} \mathrm{O}_{4}$.

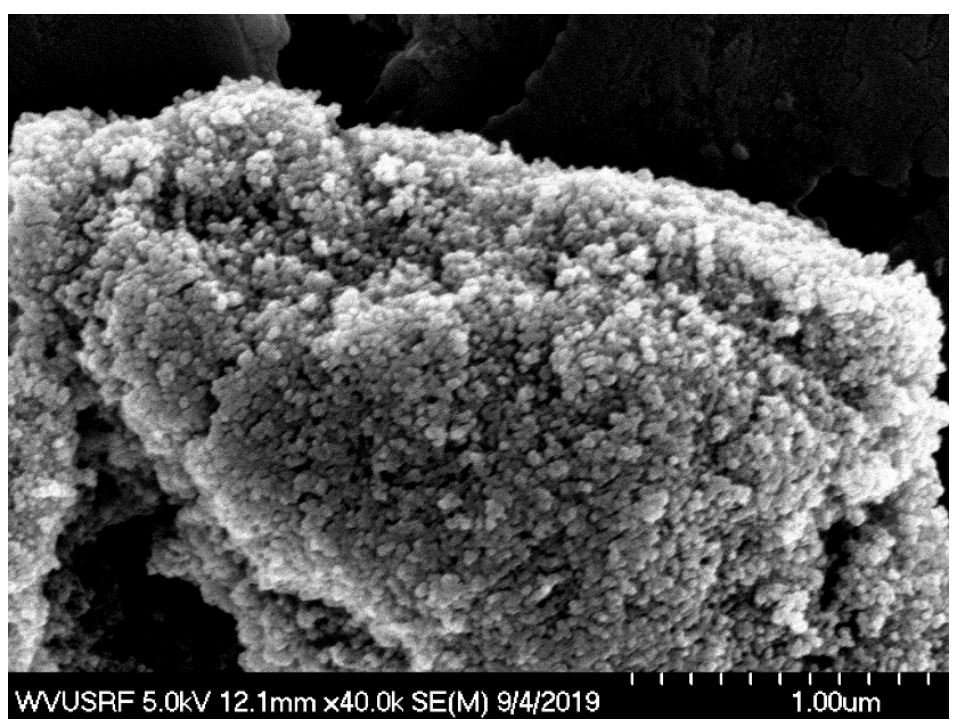

Figure 3.2: $\mathrm{SEM}$ image of preliminary $\mathrm{Fe}_{3} \mathrm{O}_{4}$ nanorod synthesis results from a quick injection of ethylenediamine.

The smaller the size of the $\mathrm{Fe}_{3} \mathrm{O}_{4}$ nanoparticles would also increase their attraction to one another, forming large agglomerates, instead of individual, identifiable particles. The formation of these small clusters of spherical nanoparticles was the largest obstacle to overcome, with many experiments concluding with similar results. Finding the appropriate addition rate and SDA were key to the creation of shaped particles. The purpose of shaping the particles into specific morphologies was to test their physical properties comparatively to lab grade, stock particles. Most stock particles compared to the following experiments are nano-sized and spherical, having consistent properties. By shaping the particles atypically to stock examples, the newer particles were tested to determine how these changes would affect their dielectric properties.

\subsubsection{Effects of Changing Synthesis Conditions of $\mathrm{Fe}_{3} \mathrm{O}_{4}$ Particles}

Numerous methods were executed in the pursuit to create shaped morphologies of both micron and nano sized $\mathrm{Fe}_{3} \mathrm{O}_{4}$ catalytic particles. The ultimate purpose of creating larger, shaped particle 
was to analyze their MW reactive properties compared to that of small, amorphous particles. $\mathrm{Fe}_{3} \mathrm{O}_{4}$ typically forms these incredibly small nano spherical particles where MW irradiation is evenly induced along the entirety of the surface. By altering the size and ideally the shape of the particles into morphologies such as rods, platelets, discs, needles, etc., induction of the MW radiation would be atypical of spherical particles as the shaped particles would have different dielectric properties. This heating effect would not be uniform, instead having increased MW induction around edges, corners, and facets of these shaped particles. The increased induction elicits the potential for increased chemical conversion as these active sites have a much higher temperature than the local temperature would suggest. Some papers have reported temperatures as much as several hundreds of degrees higher than that of the local temperature. If true, shaping catalysts for MW reactor testing could prove immensely advantageous, as it would allow manufactures to operate at much lower reactor temperatures while reaping the product yield seen from traditional high temperature reactors.

All experiments used SDAs during a reduction reaction to form the shaped morphologies of the iron particles. The concentration of the SDA would play a large role in altering the morphology of any particles in the system. SDA concentration changes would either allow shaped particles to form once a certain concentration threshold was surpassed or the alterations of SDAs in turn would change the morphology simultaneously, such as what was observed in many of the $\mathrm{CaMoO}_{4}$ reactions discussed later. Other noteworthy reactionary alterations such as changes in solute concentrations, temperature, hydrothermal treatment time, reduction reaction time, and base addition rates played roles in final particle morphologies. The results from the experiments listed below are not the only tests that were completed, instead these are the results that were the most beneficial to the purpose of this thesis. 


\subsubsection{1 $\mathrm{Fe}_{3} \mathrm{O}_{4} \mathrm{Nanorods}$}

Initial iterations for creating $\mathrm{Fe}_{3} \mathrm{O}_{4}$ nanorods was tedious, as atmospheric conditions would result in the over oxidation of the particles in the $\mathrm{Fe}^{3+}: \mathrm{Fe}^{2+}$ solution during the reduction reaction. Any excess oxygen in the reactionary system would result in an irreversible formation of hematite particles that were neither shaped nor magnetic. Deoxygenated water and a thin veil of benzene laid over the top of the mixing iron solution helped prevent the formation of hematite and other non-desirable products from forming in the solution. The shape and structure of as-prepared $\mathrm{Fe}_{3} \mathrm{O}_{4}$ nanoparticles were characterized by scanning electron microscopy (SEM) and X-ray diffraction (XRD). Figure 3.3 shows the first attempts of synthesis via SEM image of the $\mathrm{Fe}_{3} \mathrm{O}_{4}$ nanorods compared against Sample 1 (S3.1.1), a successful trial in oxygen deprived environments.

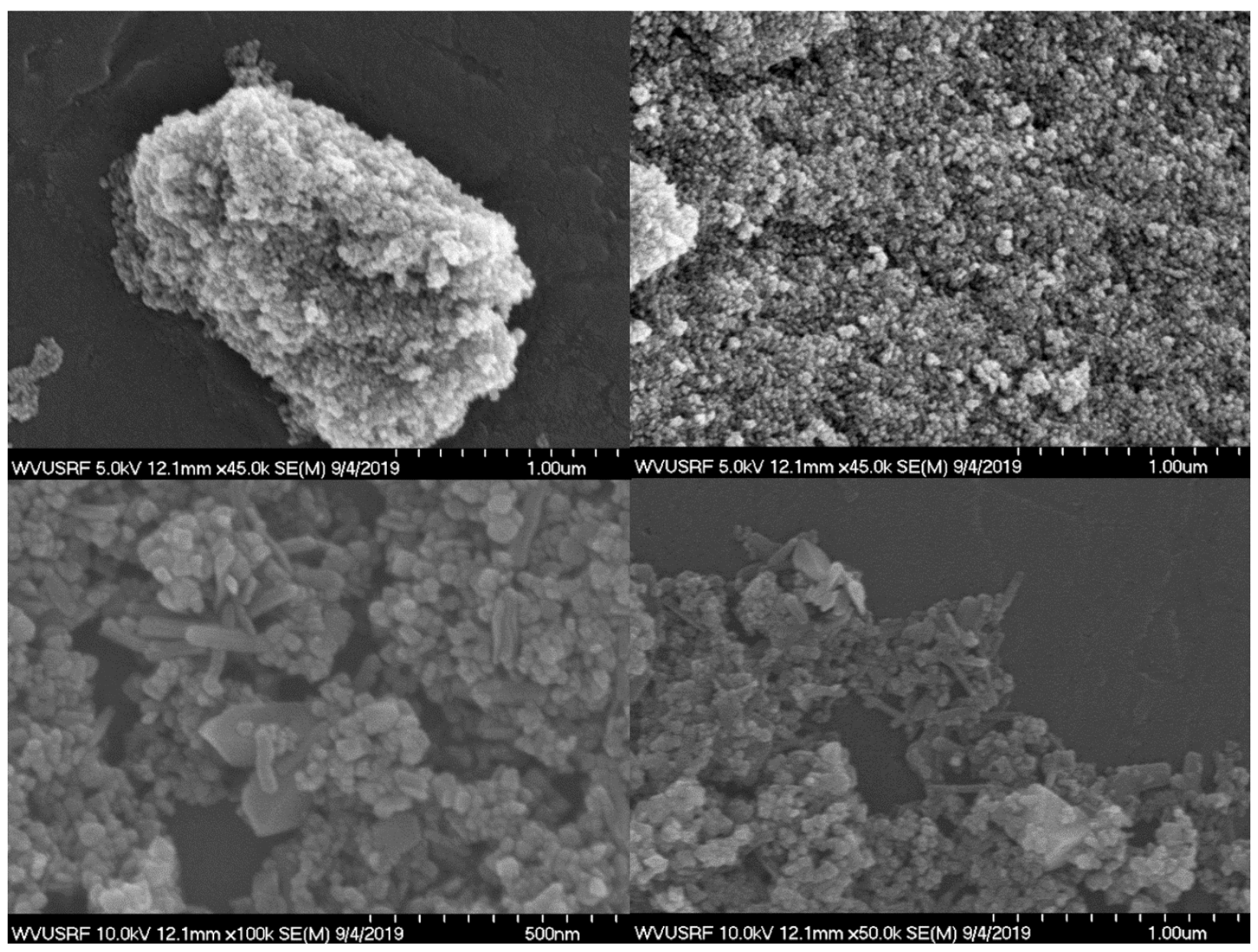


Figure 3.3: SEM imaging of initial nanorod synthesis in open atmosphere (top) vs. S3.1.1 (bottom).

Formation of nanorods was highly dependent on the reducing agent/SDA ethylenediamine as well as the ratio of $\mathrm{Fe}^{3+}$ to $\mathrm{Fe}^{2+}$ ions. Concentrations of $4 \mathrm{M}$ ethylenediamine ensured the highest concentration of these nanorod shaped particles. Lower concentration resulted in no nanorods forming due to the ethylenediamine-crystal formations not interacting, while high concentrations would reduce the iron solution rapidly and not have enough time to influence the crystal growth. The ethylenediamine would have to be slowly injected into a static solution without stirring. High stirring would introduce oxygen into the system and low rates would inactivate the surface directing of the ethylenediamine on the particles. Since alterations to the SDA concentration were sensitive, the ratio of $\mathrm{Fe}^{3+}: \mathrm{Fe}^{2+}$ was changed to alter the morphology. Ratio tests performed were to alter the final product of $\mathrm{Fe}_{3} \mathrm{O}_{4}$ and reduce residual goethite from forming in the final product, although much of this testing resulted in additional byproducts forming and/or the lack of nanorod formation. Ultimately, the yield of $\mathrm{Fe}_{3} \mathrm{O}_{4}$ nanorods were limited, only occupying a lesser portion of the total number of particles seen in the final product after numerous iterations of altering process parameters. However, some alterations did improve the quality of the final product and its purity. It was found that the ideal concentration for nanorod and magnetite yield overall was with 
an ethylenediamine concentration of $4 \mathrm{M}$. Changing the ethylenediamine concentrations outside of $4 \mathrm{M}$ would yield small impurities of goethite seen in the XRD pattern below in Figure 3.4.

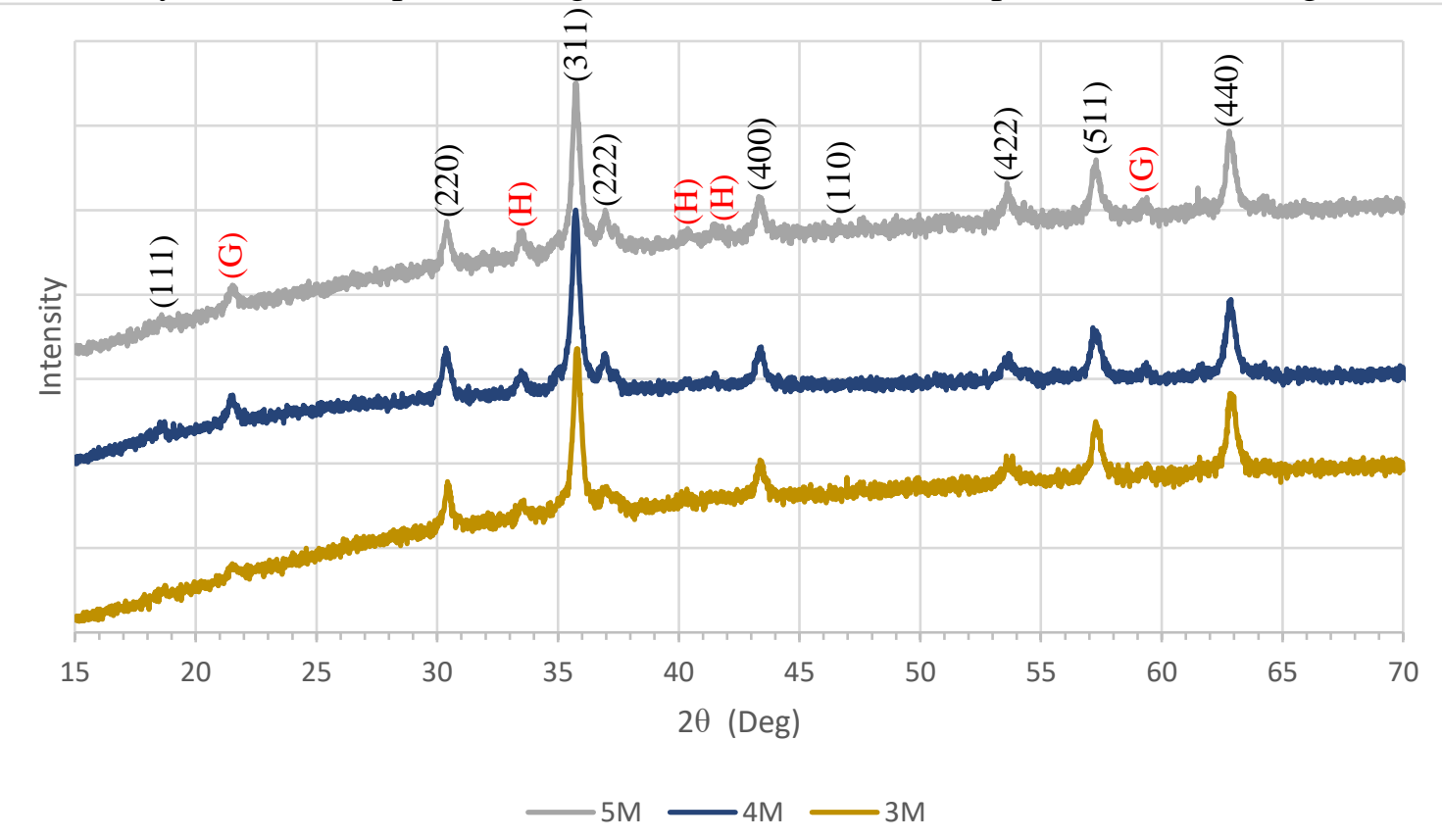

Figure 3.4: XRD patterns of $\mathrm{Fe}_{3} \mathrm{O}_{4}$ nanoparticles with altered ethylenediamine concentrations of $3 \mathrm{M}(\mathrm{S} 3.1 .2), 4 \mathrm{M}(\mathrm{S} 3.1 .1)$, and $5 \mathrm{M}(\mathrm{S} 3.1 .3)$.

A typical X-ray diffraction (XRD) pattern of the prepared nanorods using altered concentrations of ethylenediamine as both the SDA and a reducing agent is seen in Figure 3.4. The peaks at $18.7^{\circ}$, $30.4^{\circ}, 35.9^{\circ}, 37.1^{\circ}, 43.4^{\circ}, 46.3^{\circ}, 53.5^{\circ}, 57.3^{\circ}$, and $63^{\circ}$ are in good agreement with standard $\mathrm{Fe}_{3} \mathrm{O}_{4}$ diffraction patterns. All other peaks that appeared within the pattern are that of goethite and hematite, labeled $(\mathrm{G})$ and $(\mathrm{H})$, respectively. The Bragg reflection peaks are all relatively broad because of the small dimensions of the $\mathrm{Fe}_{3} \mathrm{O}_{4}$ nanocrystals. Ultimately, when comparing these tests to that of the reference they were based upon, the shape of the nanorods were consistent, but the concentration of the rods in the final product was low. SEM imagining of Wan, J. et al., 2004 final yield had significantly higher amounts of nanorod particles compared to all completed experiments reported above. Due to the lack of consistency and low concentration of shaped $\mathrm{Fe}_{3} \mathrm{O}_{4}$ nanorods, the was made decision to move on to other experiments for thin, shaped $\mathrm{Fe}_{3} \mathrm{O}_{4}$ particles. 


\subsubsection{2 $\mathrm{Fe}_{3} \mathrm{O}_{4}$ Nanoflakes}

Unlike the $\mathrm{Fe}_{3} \mathrm{O}_{4}$ nanorod experiments that used a solution containing $\mathrm{Fe}^{3+}$ and $\mathrm{Fe}^{2+}$ ions in solution, these experiments only required $\mathrm{Fe}^{2+}$ containing solutes. This lack of $\mathrm{Fe}^{3+}$ ion dependence in the initial solution reduced the effects normally seen from excess of oxygen entering the system. The reduction of $\mathrm{Fe}^{2+}$ would remain stable throughout the reaction, producing a fluffy, dark precipitate of $\mathrm{Fe}_{3} \mathrm{O}_{4}$ while completely exposed to the atmosphere. Unlike many of the other $\mathrm{Fe}_{3} \mathrm{O}_{4}$ experiments completed, this reaction was purely a coprecipitation reduction with not hydrothermal treating after the reaction to obtain nano and micron sized flakes. Additionally, this reaction used sodium acetate (NaAc) as the SDA, while all other experiments used the combination of an organic surfactant and/or a surface directing base. The solution was held at $80{ }^{\circ} \mathrm{C}$ while sodium hydroxide was slowly added at a rate of $0.5 \mathrm{~mL} / \mathrm{min}$ to react the $\mathrm{Fe}^{2+}$ ions gradually as they were directed by the dissolved NaAc. NaAc concentration in the solution played the largest role regarding the end $\mathrm{Fe}_{3} \mathrm{O}_{4}$ product as seen below in Figure 3.5. 

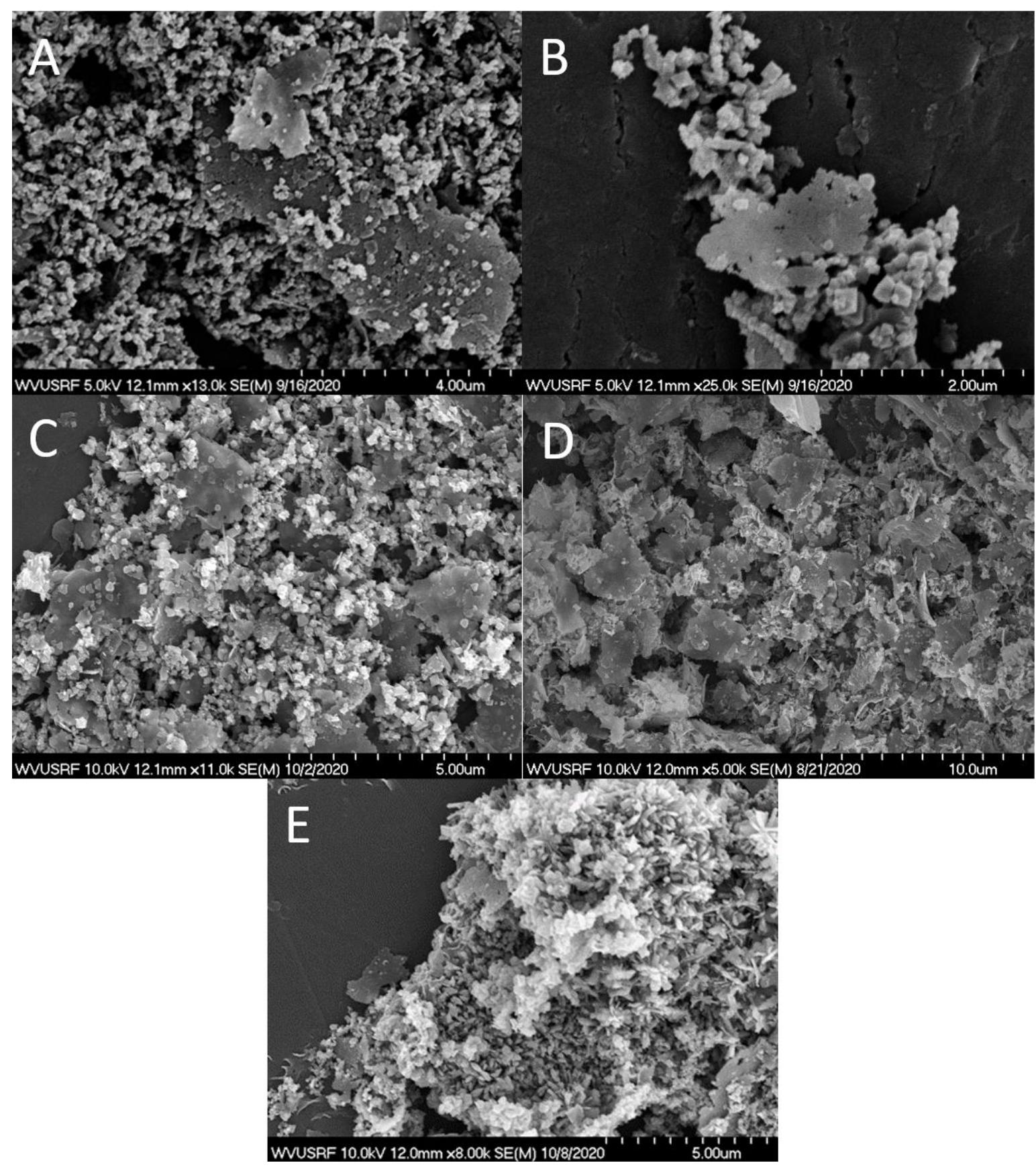

Figure 3.5: SEM images of morphological changes seen in $\mathrm{Fe}_{3} \mathrm{O}_{4}$ only by altering the concentration of sodium acetate: $322 \mathrm{mg}$ (S3.2.1), $270 \mathrm{mg}$ (S3.2.2), $238 \mathrm{mg}$ (S3.2.3), $222 \mathrm{mg}$ (S3.2.4), and $195 \mathrm{mg}(\mathrm{S} 3.2 .5)$.

Higher concentrations of NaAc in solution would increase the number of cubic/spherical particles while correspondingly reducing the platelets-like particles. The remaining platelet particles also 
show signs of degradation comparatively to the first test as voids are seen all throughout them. These voids are of similar size to the surrounding cubic and spherical particles, suggesting that the increase in these morphologies is the result of the platelet microstructure disintegration from a poor adherence to the SDA. However, at lower concentrations, NaAc begins to form more of the platelet/flake-like microstructures as well as the introduction of needle-shaped, "urchin" particles that were seen in limited numbers. Both the increase in the platelet and needle morphologies were seen as advantageous towards the design of thin particles, so the concentration was then kept at $195 \mathrm{mg}$ of $\mathrm{NaAc}$ for the remainder of synthesis tests while other parameters were adjusted. Adjusting the stirring speed during the reaction was another parameter alteration that led to an increase in platelet particles. Reducing the speed from $300 \mathrm{rpm}$ to $150 \mathrm{rpm}$ help the formation of platelets as well as reducing the presence of the smaller cubic/spherical particles and concurrently the voids seen in the platelets as seen in Figure 3.6.

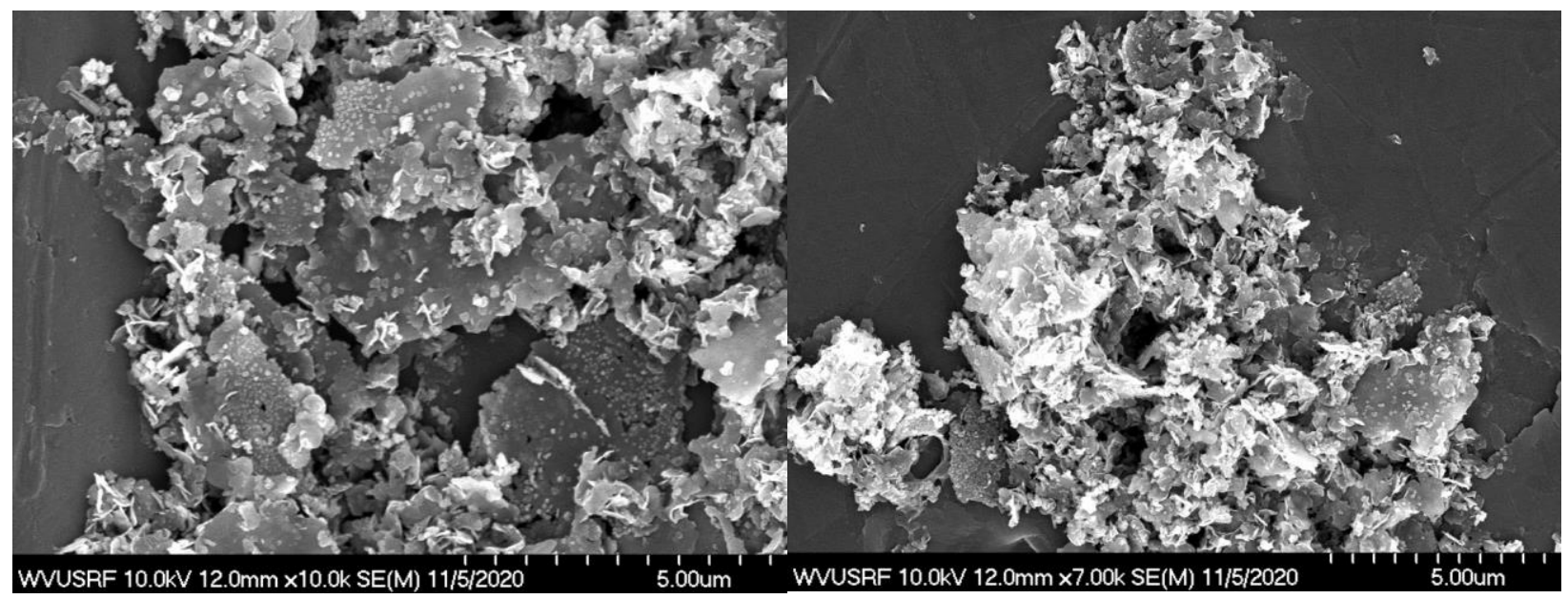

Figure 3.6: $\mathrm{SEM}$ imaging of $\mathrm{Fe}_{3} \mathrm{O}_{4}$ particle using $195 \mathrm{mg}$ of $\mathrm{NaAc}$ while stirring at $150 \mathrm{rpm}$ for 3 hours (S3.2.6).

Slower stirring speeds were also advantageous in the formation of nanorod particles $\mathrm{Fe}_{3} \mathrm{O}_{4}$, which influenced the decision to make this adjustment. Lastly, extending the coprecipitation reaction time 
from 3 to 6 hours finally eliminated the presence of small cubic/spherical particles, leaving only $\mathrm{Fe}_{3} \mathrm{O}_{4}$ nano/microflakes as the dominant morphology in Sample 7 (S3.2.7) as seen in Figure 3.7.

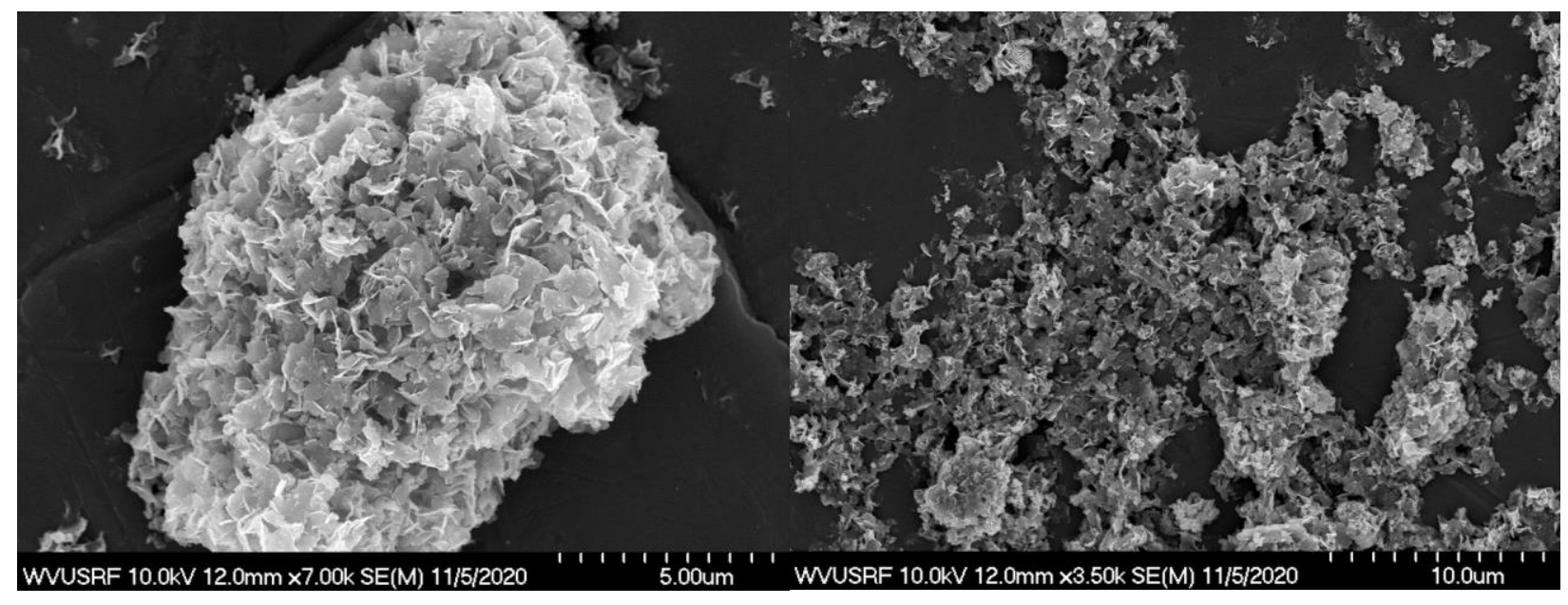

Figure 3.7: $\mathrm{SEM}$ imaging of $\mathrm{Fe}_{3} \mathrm{O}_{4}$ particle using $195 \mathrm{mg}$ of $\mathrm{NaAc}$ while stirring at $150 \mathrm{rpm}$ for 6 hours (S3.2.7).

A typical X-ray diffraction (XRD) pattern of the prepared flakes using altered concentrations of $\mathrm{NaAc}$ as an SDA and adjusting the stirring speed and reaction time seen in Figure 3.8. The peaks at $18.7^{\circ}, 30.4^{\circ}, 35.9^{\circ}, 37.1^{\circ}, 43.4^{\circ}, 53.5^{\circ}, 57.3^{\circ}$, and $63^{\circ}$ are in good agreement with standard $\mathrm{Fe}_{3} \mathrm{O}_{4}$ diffraction patterns. All other peaks that appeared within the pattern are that of goethite and hematite, labeled $(\mathrm{G})$ and $(\mathrm{H})$, respectively.

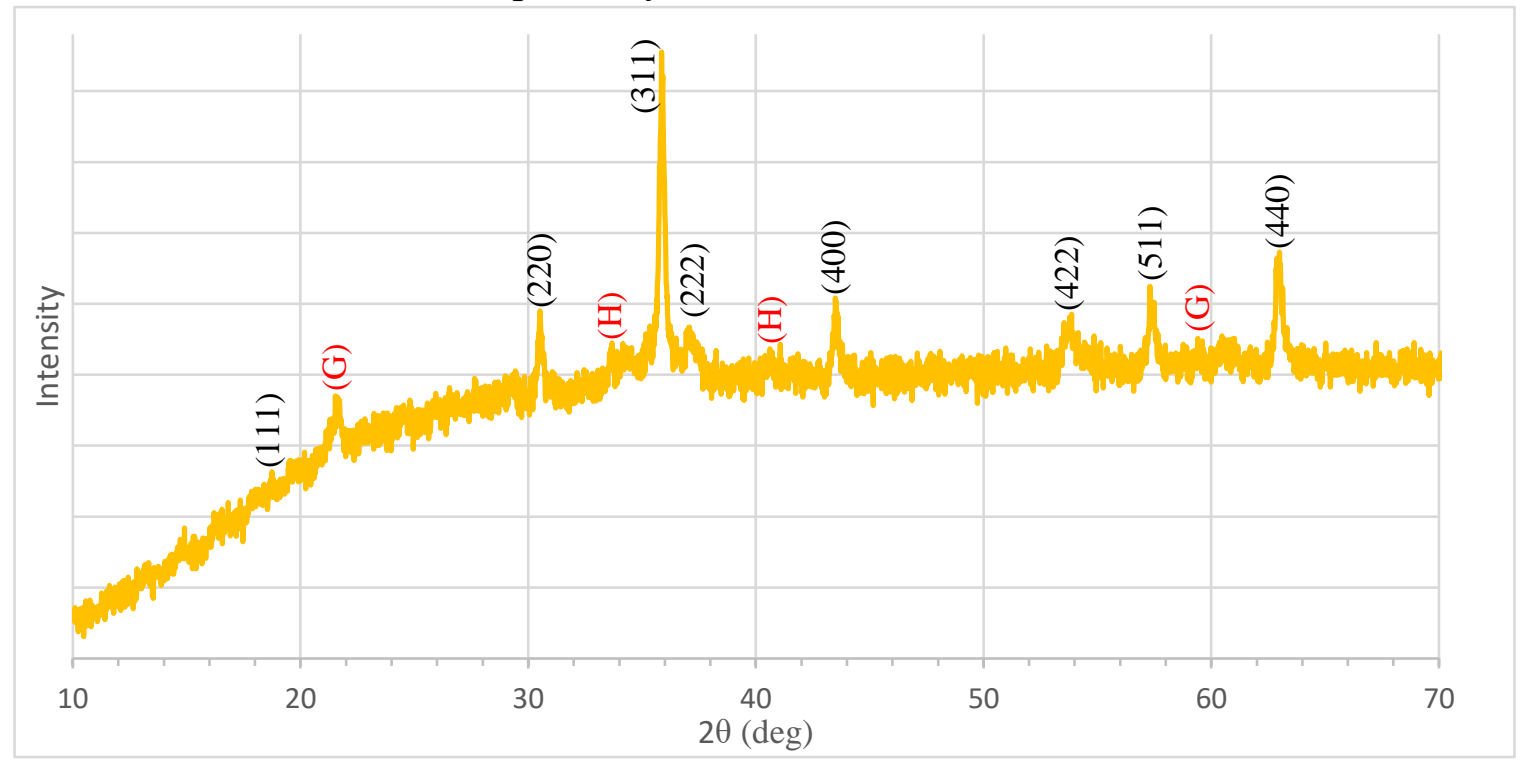


Figure 3.8: XRD pattern of $\mathrm{Fe}_{3} \mathrm{O}_{4}$ nanoparticles (S3.2.7) with a NaAc concentration of $195 \mathrm{mg}$, stirred at 150 rpm for 6 hours.

\subsubsection{3 $\mathrm{Fe}_{3} \mathrm{O}_{4}$ Micro Platelets and Octahedrons}

Since the procurement of platelet and flake-like particles were easier to manifest in comparison to needle or rod morphologies, these continued to be the focal point of $\mathrm{Fe}_{3} \mathrm{O}_{4}$ synthesis reactions. The next goal would be to observe any reactionary differences in particles with similar morphologies, but with larger particle sizes. Larger, micron-sized particles that are closer to MW wavelengths may interact with these wavelengths differently than their nano-sized counterparts when in the MW reactor. Additionally, larger structures have been proven to be easier to model with particle dispersion software, as the mathematics become incredibly complex when the size of the particles enter nano-sized territory. To achieve micron sized $\mathrm{Fe}_{3} \mathrm{O}_{4}$ particles, a twostep hydrothermal process was implemented. The first step for the procurement of the micro platelets was to reduce iron (III) acetylacetonate with sodium hydroxide, suspended in $100 \mathrm{ml}$ of triethylene glycol (TEG) acting as the SDA for this reaction before placing these particles within the Teflon lined hydrothermal vessel for 4 hours at $200{ }^{\circ} \mathrm{C}$. This initial hydrothermal treatment was to partially reduce and synthesize the $\mathrm{Fe}_{2} \mathrm{O}_{3}$ precursor and grow the platelet shaped particles through the first hydrothermal treatment. $\mathrm{Fe}_{2} \mathrm{O}_{3}$ has been shown more frequently in literature to have the ability to grow larger, shaped particles when compared to supporting documents for $\mathrm{Fe}_{3} \mathrm{O}_{4}$ synthesis. Thereafter, the newly produced $\mathrm{Fe}_{2} \mathrm{O}_{3}$ platelets would be fully reduced using sodium borohydride followed by another hydrothermal treatment of 12 hours at $160^{\circ} \mathrm{C}$. The longer treatment was to ensure the reaction was completed fully, theoretically leaving little to no residual $\mathrm{Fe}_{2} \mathrm{O}_{3}$ in the magnetite product. Initial synthesis attempts yielded high amounts of $\mathrm{Fe}_{2} \mathrm{O}_{3}$ in the final product while also showing weak ferromagnetism when introduced to a magnet. XRD analysis seen in 
Figure 3.9 also confirms the presence of hematite as well as other residual undesirable products seen within the pattern.

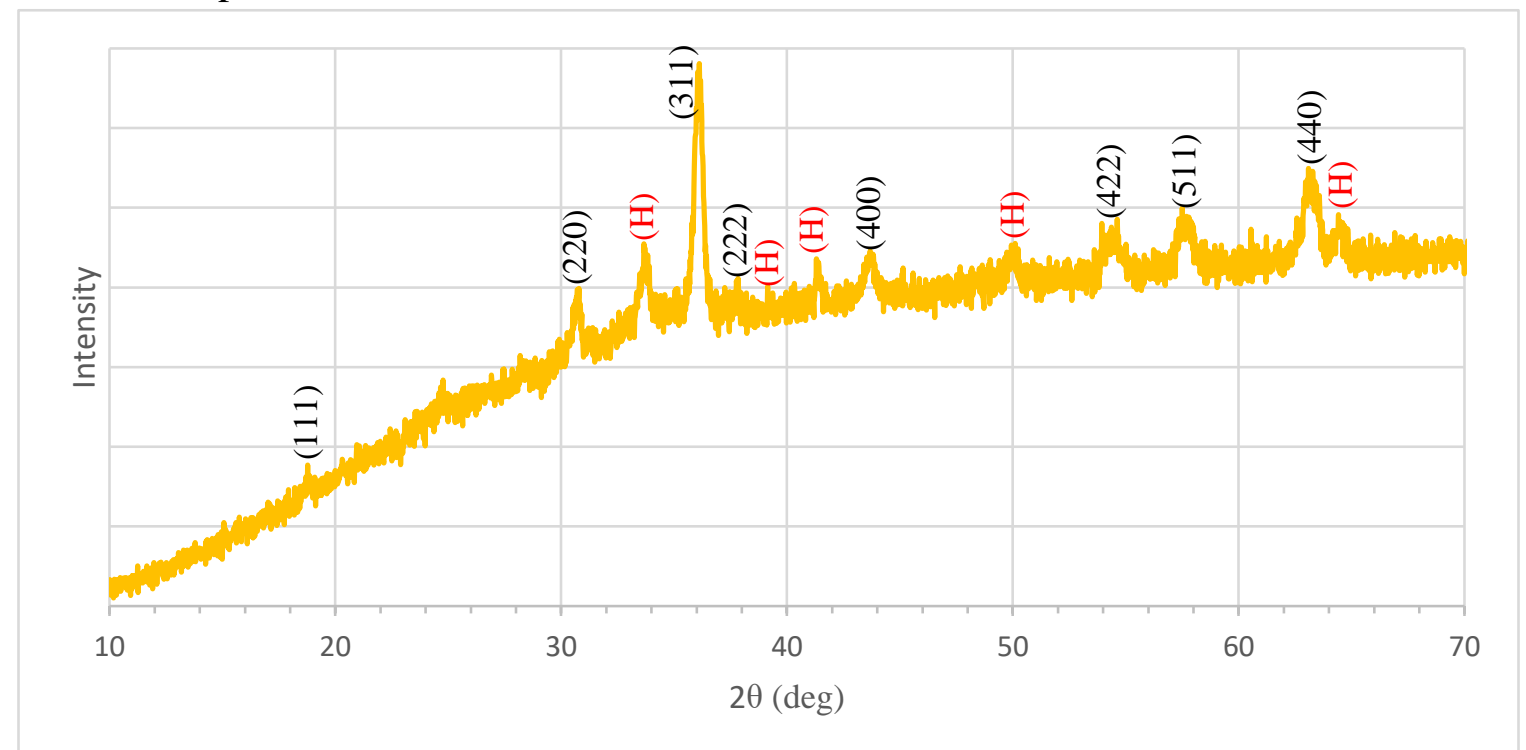

Figure 3.9: XRD pattern of initial synthesis attempt (S3.3.1) at micron sized $\mathrm{Fe}_{3} \mathrm{O}_{4}$ platelets showing traces of residual $\mathrm{Fe}_{2} \mathrm{O}_{3}$.

The peaks at $18.7^{\circ}, 30.4^{\circ}, 35.9^{\circ}, 37.1^{\circ}, 43.4^{\circ}, 53.5^{\circ}, 57.3^{\circ}$, and $63^{\circ}$ are in good agreement with standard $\mathrm{Fe}_{3} \mathrm{O}_{4}$ diffraction patterns. All other peaks that appeared within the pattern are that of hematite $(\mathrm{H})$. Although the majority diffraction peaks indicate that magnetite is dominant, the hematite left over from the initial hydrothermal treatment is too large to ignore, prompting the need for an adjustment to the process. SEM imaging confirms the residual presence of the smoother hematite micro-platelets as most of the plates show signs of magnetite partially forming after the $\mathrm{NaBH}_{4}$ reduction. Figure 3.10 compares images of Sample 1 (S3.3.1) vs. $\mathrm{Fe}_{2} \mathrm{O}_{3}$ and $\mathrm{Fe}_{3} \mathrm{O}_{4}$ examples from the literature. 


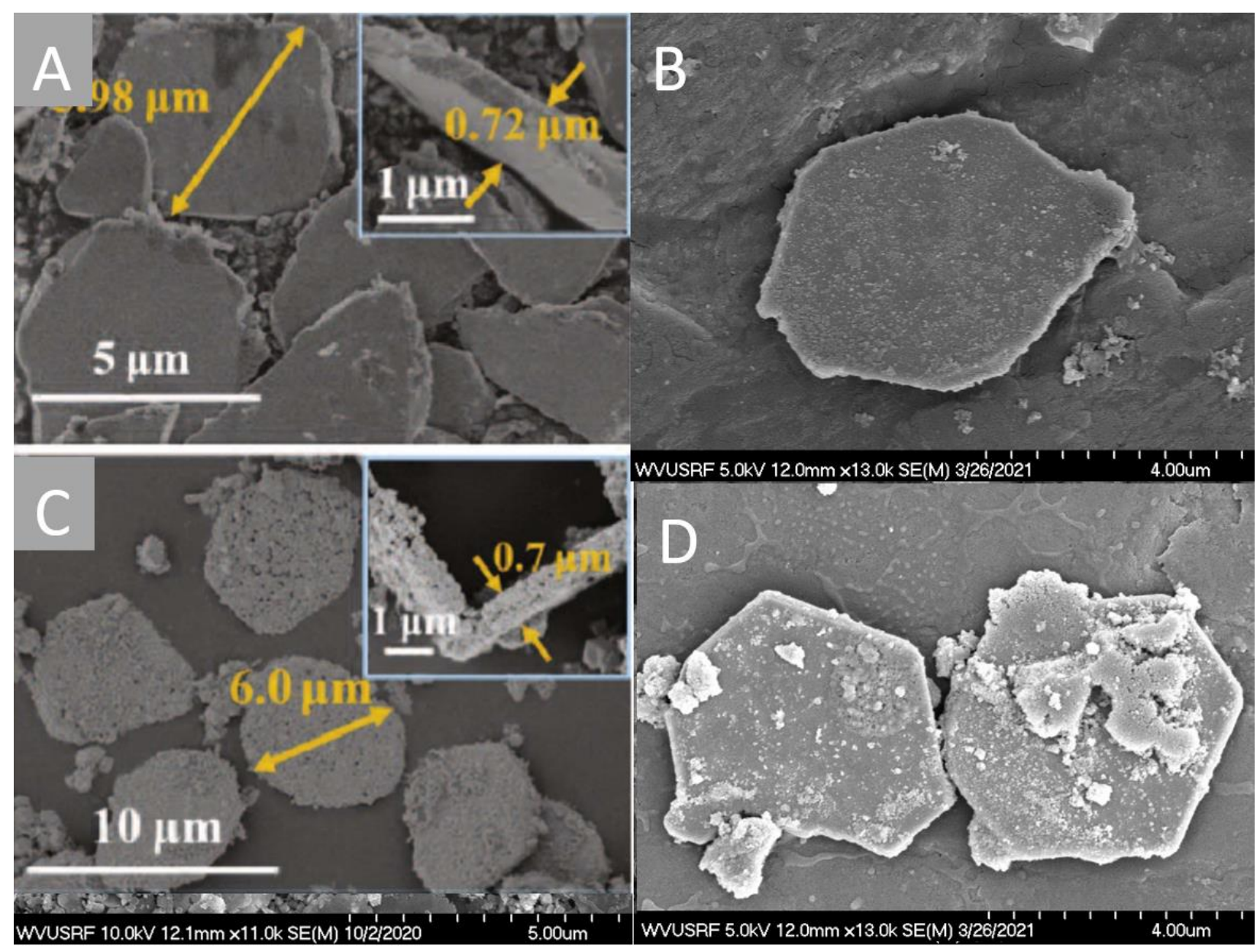

Figure 3.10: $\mathrm{SEM}$ imaging of $\mathrm{Fe}_{2} \mathrm{O}_{3}(\mathrm{~A})$ and $\mathrm{Fe}_{3} \mathrm{O}_{4}(\mathrm{C})$ micro-platelet formations from literature vs. S3.3.1 of $\mathrm{Fe}_{2} \mathrm{O}_{3}$ (B) and $\mathrm{Fe}_{3} \mathrm{O}_{4}$ (D) micro-platelet formation (Zhao et al. 2018).

SEM imaging suggested the surface of the $\mathrm{Fe}_{2} \mathrm{O}_{3}$ micro-platelets were not fully reduced in the presence of $\mathrm{NaBH}_{4}$. The surface was expected to have a fully textured, somewhat bumpy depiction, although many of the particles still exhibited areas of smooth surfaces indicating the continued presence of $\mathrm{Fe}_{2} \mathrm{O}_{3}$. To correct this problem, the amount of $\mathrm{NaBH}_{4}$ was increased slightly for Sample 2 (S3.3.2) and Sample 3 (S3.3.3) to $756 \mathrm{mg}$ and $800 \mathrm{mg}$, respectively, in $60 \mathrm{ml}$ of DI water. Additionally, both sample sizes were doubled, increasing all reagents used outside the amount of TEG in each experimental run. S3.3.2 doubled the amount of TEG from 50 to $100 \mathrm{ml}$ used while S3.3.3 tripled the amount to $150 \mathrm{ml}$ to determine if this SDA concentration directly controlled 
particle shape formation or if another factor was at play. All other variables were kept constant. After the second hydrothermal reduction using the slightly greater concentration of $\mathrm{NaBH}_{4}$, the particles were notably darker in color and had a greater affinity to the magnet during the washing process where magnetic separation was utilized. XRD analysis seen in Figure 3.11 reaffirms the presence of $\mathrm{Fe}_{3} \mathrm{O}_{4}$ as well as the near complete absence of residual hematite and other nondesirable byproducts of the first reduction.

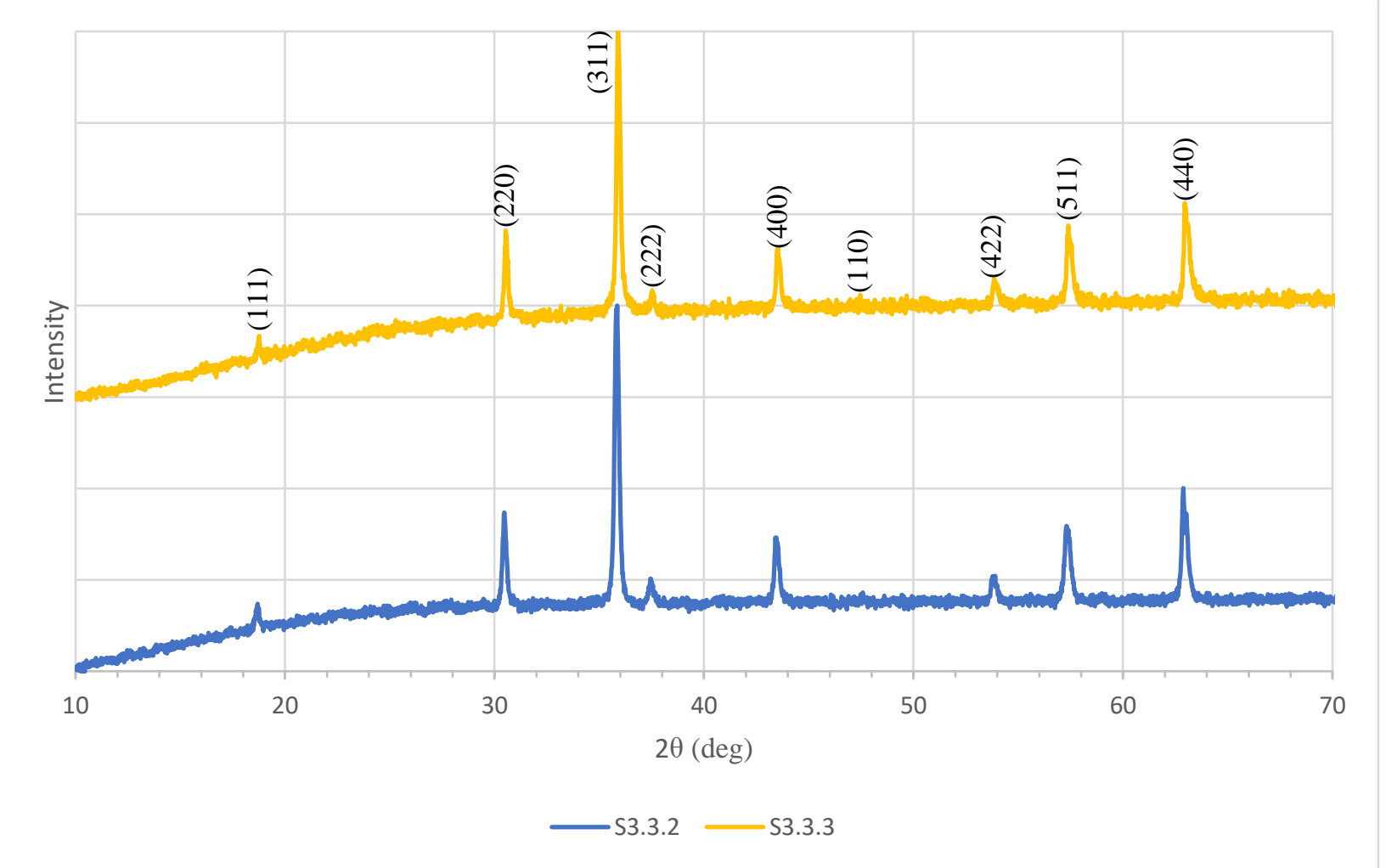

Figure 3.11: XRD pattern of S3.3.2 (octahedrons) and S3.3.3 (platelets)

Unlike previously, these XRD patterns of S3.3.2 and S3.3.3 at $18.7^{\circ}, 30.4^{\circ}, 35.9^{\circ}, 37.1^{\circ}, 43.4^{\circ}$, $46.3^{\circ}, 53.5^{\circ}, 57.3^{\circ}$, and $63^{\circ}$ are in good agreement with standard $\mathrm{Fe}_{3} \mathrm{O}_{4}$ diffraction patterns. There are no other peaks that would suggest any contamination of impurities such as goethite or hematite. Previous experiments normally showed the presence of goethite in the final product, which is also absent from this data. Examining the SEM images of these particles confirms the importance of increasing the concentration of $\mathrm{NaBH}_{4}$ to fully reduce the surface of the samples into pure $\mathrm{Fe}_{3} \mathrm{O}_{4}$, 
as all examples exhibit the traditional textured nature seen in all confirmed samples. Also, changing the concentration of TEG was confirmed to be the main contributor in altering particle morphology as seen in Figure 3.12.

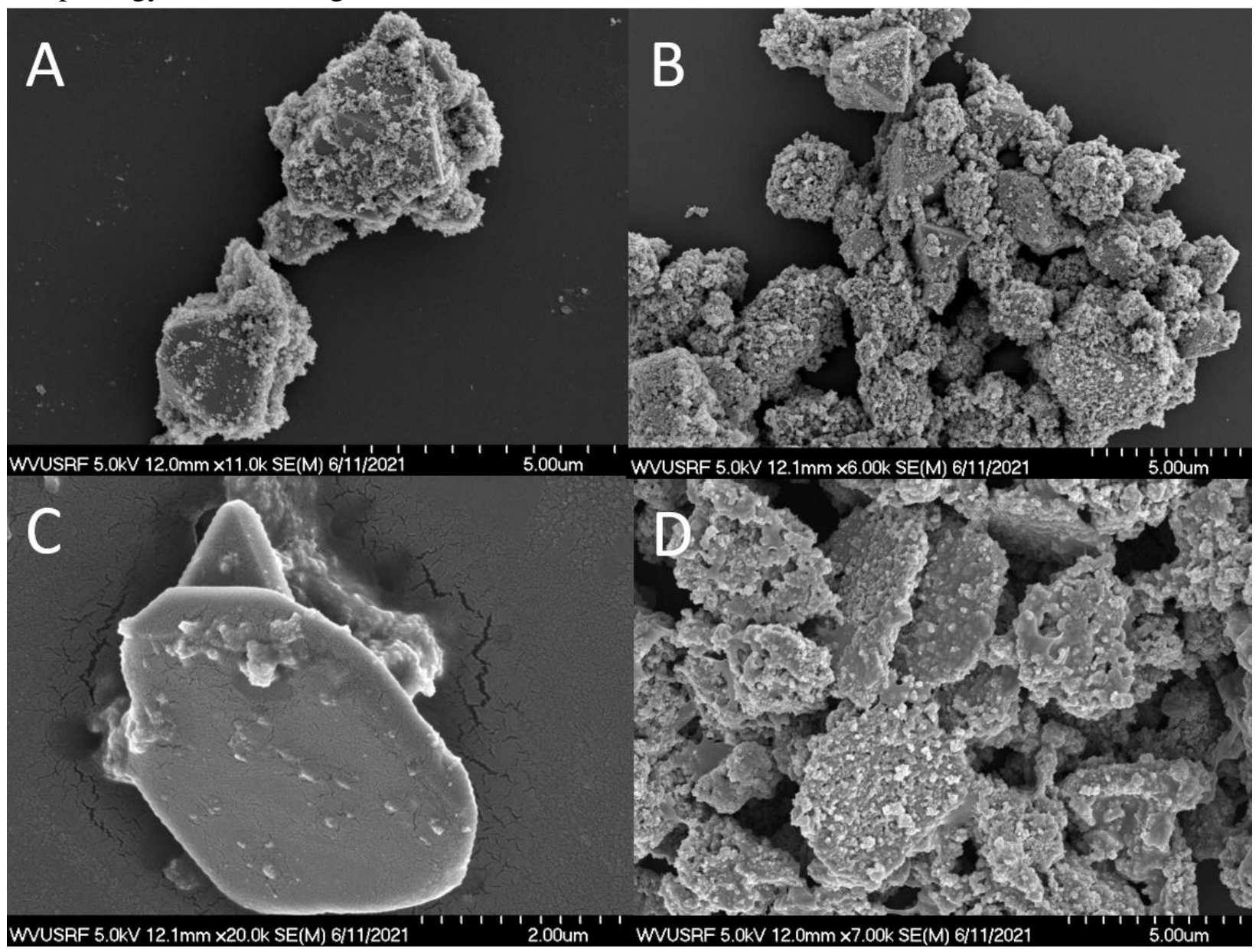

Figure 3.12: $\mathrm{SEM}$ images of $\mathrm{Fe}_{2} \mathrm{O}_{3}$ (left) and $\mathrm{Fe}_{3} \mathrm{O}_{4}$ (right) of S3.3.2 micro-octahedrons (A, B) and S3.3.3 micro platelet (C, D) formations.

S3.3.2 had all reagent concentrations doubled to increase the final yield of the sample, lending to the idea of keeping the particles size and final morphology constant since the SDA was not changed. S3.3.3 was meant test for divergence from the platelet morphology if possible, by increasing the SDA concentration by $50 \%$ when scaling these tests. However, the final morphology of the two samples contradicted this theory, as S3.3.2 showed uniform, octahedral particles and 
S3.3.3 showed the same platelet-like particles previously seen, despite the higher SDA concentration. Both samples showed the textured nature of $\mathrm{Fe}_{3} \mathrm{O}_{4}$ as expected from the XRD patterns, previous synthesis attempts, and supporting articles. This change in morphology was not caused by slightly different, higher concentrations of $\mathrm{NaBH}_{4}$ since the same morphologies were seen in the $\mathrm{Fe}_{2} \mathrm{O}_{3}$ particles directly after the initial hydrothermal treatment. The additional $\mathrm{NaBH}_{4}$ however did contribute to the complete conversion of $\mathrm{Fe}_{2} \mathrm{O}_{3}$ to $\mathrm{Fe}_{3} \mathrm{O}_{4}$, unlike S3.3.1. Contamination was also ruled out as a reason for the unexpected changed in the particle morphology since different hydrothermal vessels were used and the morphologies of the preceding $\mathrm{Fe}_{2} \mathrm{O}_{3}$ products are consistent with the final morphologies. Since all other variables were kept constant for the entirety of the hydrothermal process, it was concluded that simply doubling the reagent concentrations did not lend to the notion that the morphologies would be kept constant. Instead, the relation of the TEG concentration and final morphology of the $\mathrm{Fe}_{2} \mathrm{O}_{3}$ particles were not considered linear as initially anticipated. The relation was instead assumed to be regarding other variables such as $\mathrm{NaOH}$ addition rates or stirring speeds, as these were not as closely monitored between the two experiments compared to other variables. Both samples were stirred between $600-800 \mathrm{rpm}$ for the same time, although the $\mathrm{NaOH}$ addition rate for $\mathrm{S} 3.3 .2$ was the same as $\mathrm{S} 3.3 .1$ at $1 \mathrm{~mL} / \mathrm{min}$, while $\mathrm{S} 3$ 's addition rate was done drop-wise without a flow regulator. Although these are immoderately different, this would also not lend the changes seen in the morphology, since S3.3.2 was completed the same as S3.3.1 yet exhibited octahedral particles instead of platelets. Therefore, it was concluded that the difference was most likely due to the unregulated stirring speeds that lead to the change in final morphologies, since this was a common issue with previous shaped magnetite synthesis methods. 


\subsubsection{Synthesis of Calcium and Strontium Molybdate Particles and Morphological Changes}

The morphology and size of the $\mathrm{CaMoO}_{4}$ and $\mathrm{SrMoO}_{4}$ microparticles were able to be selectively controlled by altering one or more of several growth variables. Altering the alkaline earth metal reagents and the concentrations of SDAs had the largest effect on the morphological outcome of the particles. Temperature conditions and reactor time also played a part in manipulating the size of the final particles. Facilitating the growth of shaped, microparticles was less taxing than that of $\mathrm{Fe}_{3} \mathrm{O}_{4}$, where small alterations to the synthesis recipe obtained desirable morphologies for dielectric and MW reactor studies compared to that of stock particles. $\mathrm{CaMoO}_{4}$ morphologies included needle, platelet, platelet clusters, and multi-crystalline doughnut shaped particles and $\mathrm{SrMoO}_{4}$ experiments concluded with large, faceted, spherical clusters, much larger than stock examples.

\subsubsection{1 $\mathrm{CaMoO}_{4}$ Needles, Doughnuts and Platelets}

Unlike the synthesis of shaped, micron sized $\mathrm{Fe}_{3} \mathrm{O}_{4}, \mathrm{CaMoO}_{4}$ was easily manipulated by tweaking several synthesis parameters, namely the amount of SDA and calcium chloride $\left(\mathrm{CaCl}_{2}\right)$ reagent used. N, N-dimethylacetamide (DMA) was the SDA implemented for these experiments, and its concentration in the system was the defining variable for final morphology of $\mathrm{CaMoO}_{4}$. Initially, 
$12 \mathrm{~mL}$ of DMA was mixed with $68 \mathrm{~mL}$ of DI water, resulting in both needle and platelet clusters in the result as seen in Figure 3.13.

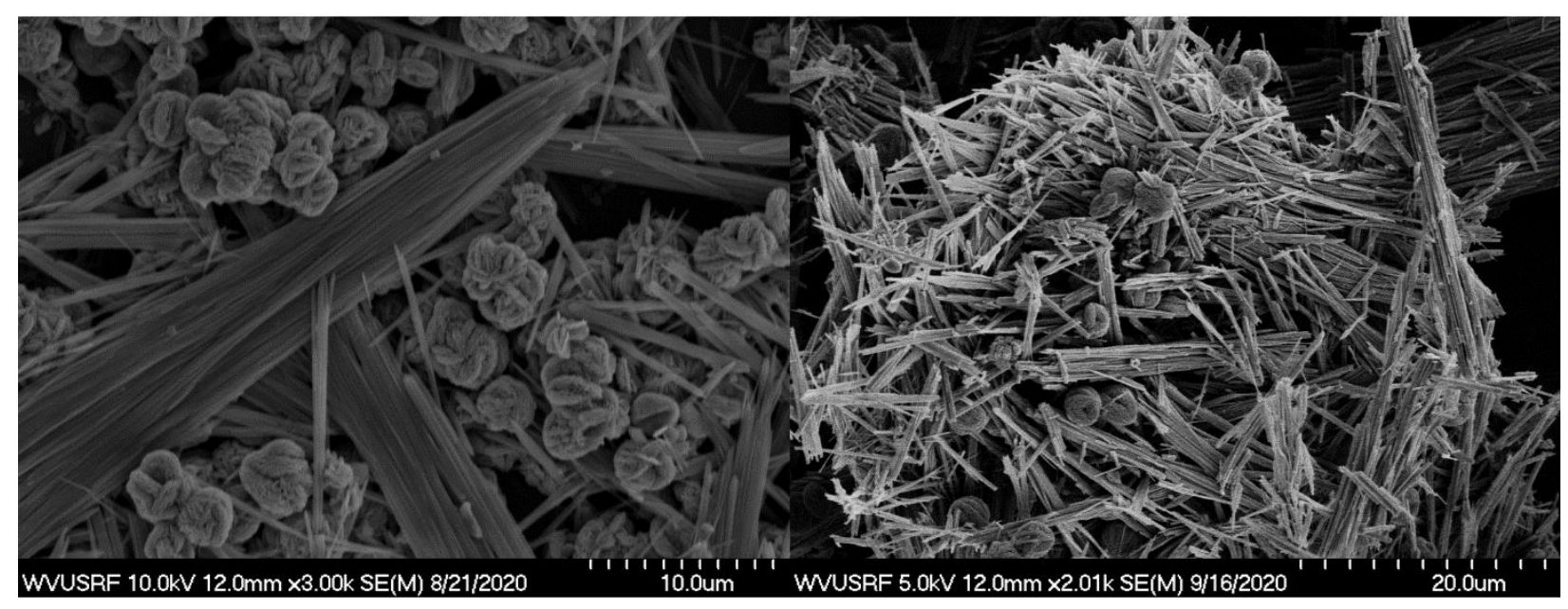

Figure 3.13: SEM images of $\mathrm{CaMoO}_{4}$ clusters and needles, S3.4.1 (left) and S3.4.1 repeated (right).

This duel morphological result was easily repeatable and XRD analysis did not show any indication that any undesirable products were created during the reaction, ruling out possibility of diverging side reactions or contamination in the product.

A 33\% lower concentration of DMA $(8 \mathrm{~mL})$ would result in larger, doughnut shaped, multicrystalline structures, 3-5 microns in size while higher concentrations yielded smaller, singlecrystalline platelets that would cluster into either planar or sphere-like particles. The ratio of DMA to DI water was kept at 1:4, $16 \mathrm{~mL}$ of DMA, as this concentration would consistently yield micron sized particles with a desirable platelet-like structure. Next, the hydrothermal treatment time was reduced from 30 minutes to 20 minutes to achieve smaller, individual platelet particles. However, the shorter treatment time caused two problem; XRD analysis suggested impurities were present 
in the final sample and SEM imaging indicated larger micron sized needles became the dominant morphology with broken, irregular platelets seen in the final product in Figure 15.

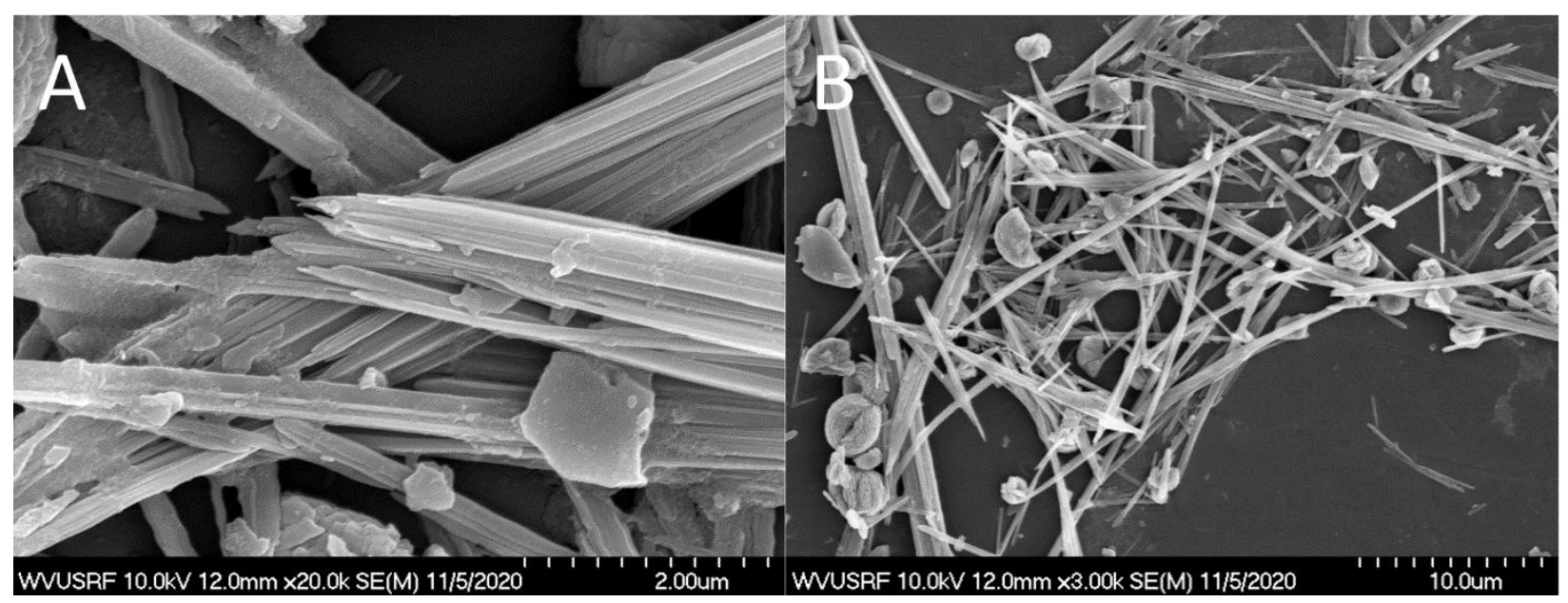

Figure 3.14: SEM images of Sample 4 (S3.4.4) ( $\left.\mathrm{CaMoO}_{4}\right)$ with a needle dominant morphology.

Pivoting from this, the temperature was instead reduced by $25 \%$ from $180{ }^{\circ} \mathrm{C}$ to $140{ }^{\circ} \mathrm{C}$ to yield smaller particles, although this too ended with platelet-like clusters looking like a fusion between the previously synthesized doughnut and single-crystalline platelet clusters as seen in the Figure 3.15 comparison. 


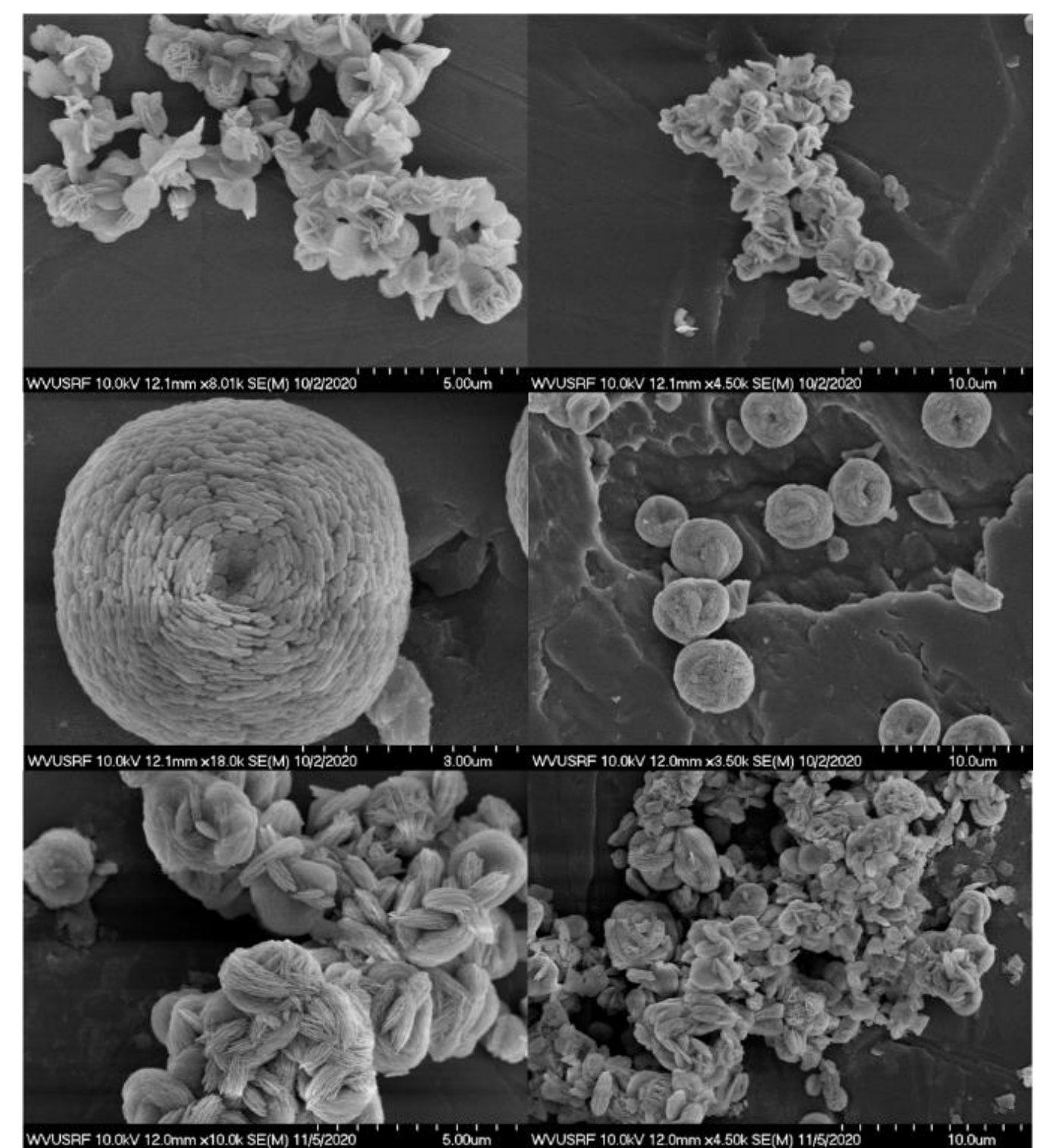

Figure 3.15: SEM images of $\mathrm{CaMoO}_{4}$ Sample 2 (S3.4.2) (top) and Sample 3 (S3.4.3) (middle)

compared to Sample 5 (S3.4.5) (bottom).

These formations of needle shaped $\mathrm{CaMoO}_{4}$ could have also been occurring due to rapid cooling of the hydrothermal vessel, causing similar morphologies to crash out of the solution like what is seen in Tishima et. al. To avoid previous morphologies from forming, the temperature and time of the hydrothermal treatment were kept constant for future reactions. Sample 6 (S3.4.6) finally yielded uniform, flat, platelet clusters after increasing the amount of $\mathrm{CaCl}_{2}$ by $50 \%$. The increase provided the reaction in the hydrothermal vessel with a higher concentration of reaction sites for 
the ammonium molybdate to react with $\mathrm{CaCl}_{2}$. More reactions taking place throughout the system directly affected the amount of nucleation sites for precipitates to crash out of solution, instead of growing off fewer, larger particles like previous experiments. The final product yielded flat, platelet clusters of clean $\mathrm{CaMoO}_{4}$ as seen in Figure 3.16 and confirmed via XRD analysis compared to previous synthesis attempts in Figure 3.17.

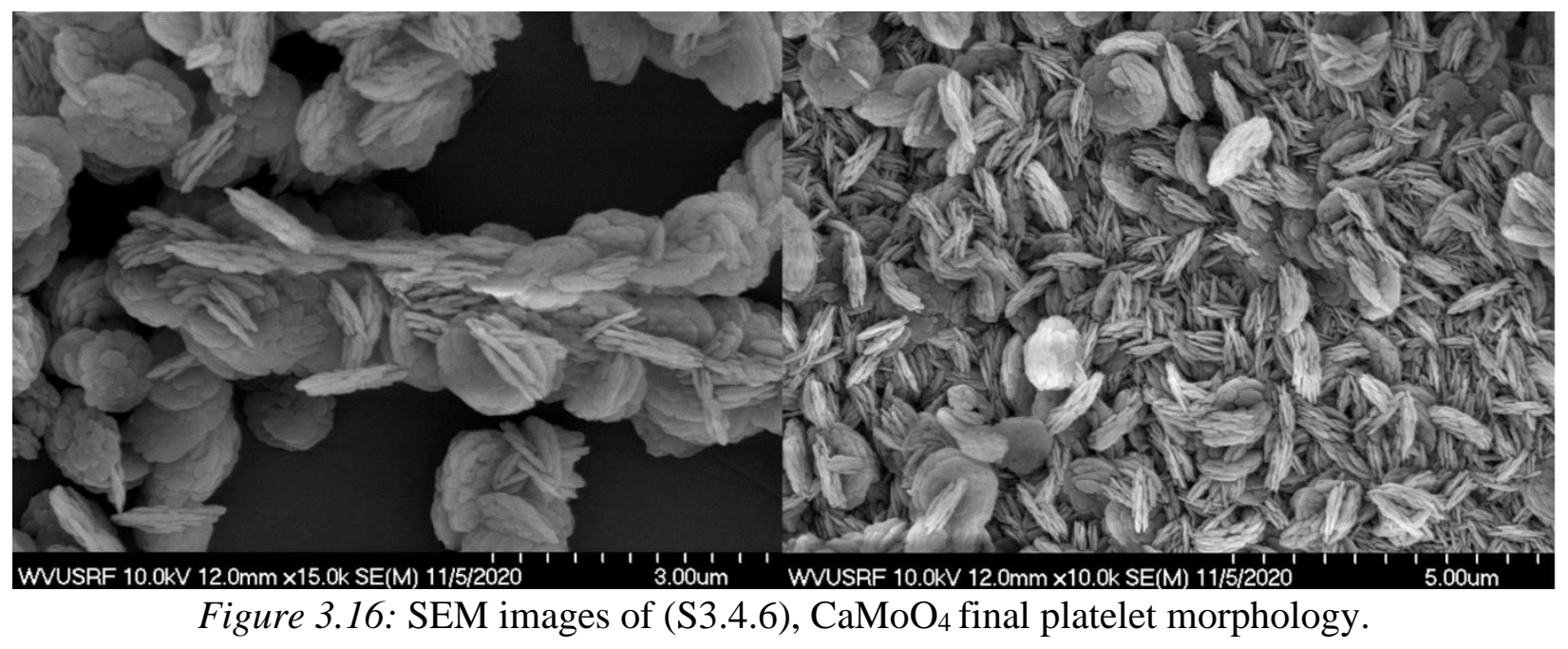




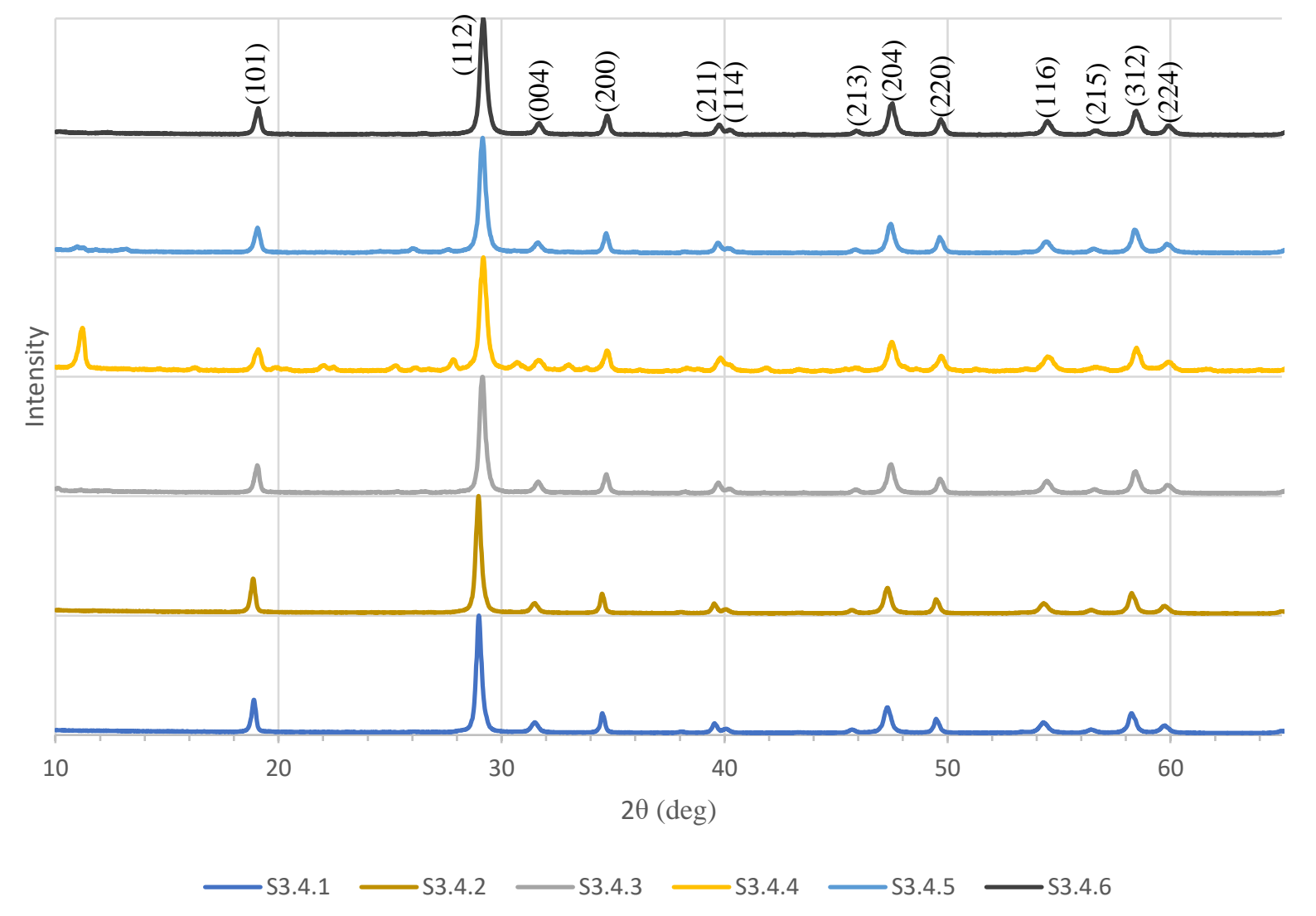

Figure 3.17: XRD pattern comparison of different morphological samples of $\mathrm{CaMoO}_{4}, \mathrm{~S} 3.4 .1$ through S3.4.6.

These XRD peak patterns at $18.8^{\circ}, 29.5^{\circ}, 31.4^{\circ}, 34.6^{\circ}, 39.5^{\circ}, 40^{\circ}, 45.6^{\circ}, 47.4^{\circ}, 49.6^{\circ}, 54.3^{\circ}$, $56.3^{\circ}, 58.2^{\circ}$, and $59.8^{\circ}$ from samples S3.4.1 through S3.4.6, with the exception of sample S3.4.4 which contains traces amounts of impurities, are in good agreement with typical calcium molybdate patterns.

\subsubsection{2 $\mathrm{SrMoO}_{4}$ Multifaceted Micro Spheres}

Alkaline earth metal oxides have shown to process $\mathrm{CO}_{2}$ adsorption characteristic useful for any form of traditional or modified variants of the DRM process, but research on these materials is scarce. Strontium oxide itself has limited use as a catalyst but is notably effective in biodiesel production and experimental MW catalytic design (Patil et. al). Another study reports strontium 
carbonate $\left(\mathrm{SrCO}_{3}\right)$ with exposed surface of $\mathrm{SrO}$ molecules yielded 1-2\% $\mathrm{C}_{2}$ products when exposed to a 1:1 ratio of $\mathrm{CH}_{4}$ and $\mathrm{CO}_{2}$ in an electrically heated reactor at $800{ }^{\circ} \mathrm{C}$ (Aika and Nishiyama, 1989). Due to similar characteristics and synthesis methods to $\mathrm{CaMoO}_{4}$, strontium molybdate was an appropriate selection as the next MWDRM catalyst tested. $\mathrm{SrMoO}_{4}$ was ultimately the synthesized the same way as $\mathrm{CaMoO}_{4}$, using strontium nitrate and ammonium molybdate as reagents for the coprecipitation reaction. However, $\mathrm{SrMoO}_{4}$ did not require the use of an SDA, naturally creating the multi-faceted spheres after the hydrothermal processing as seen in the SEM images of Figure 3.18.

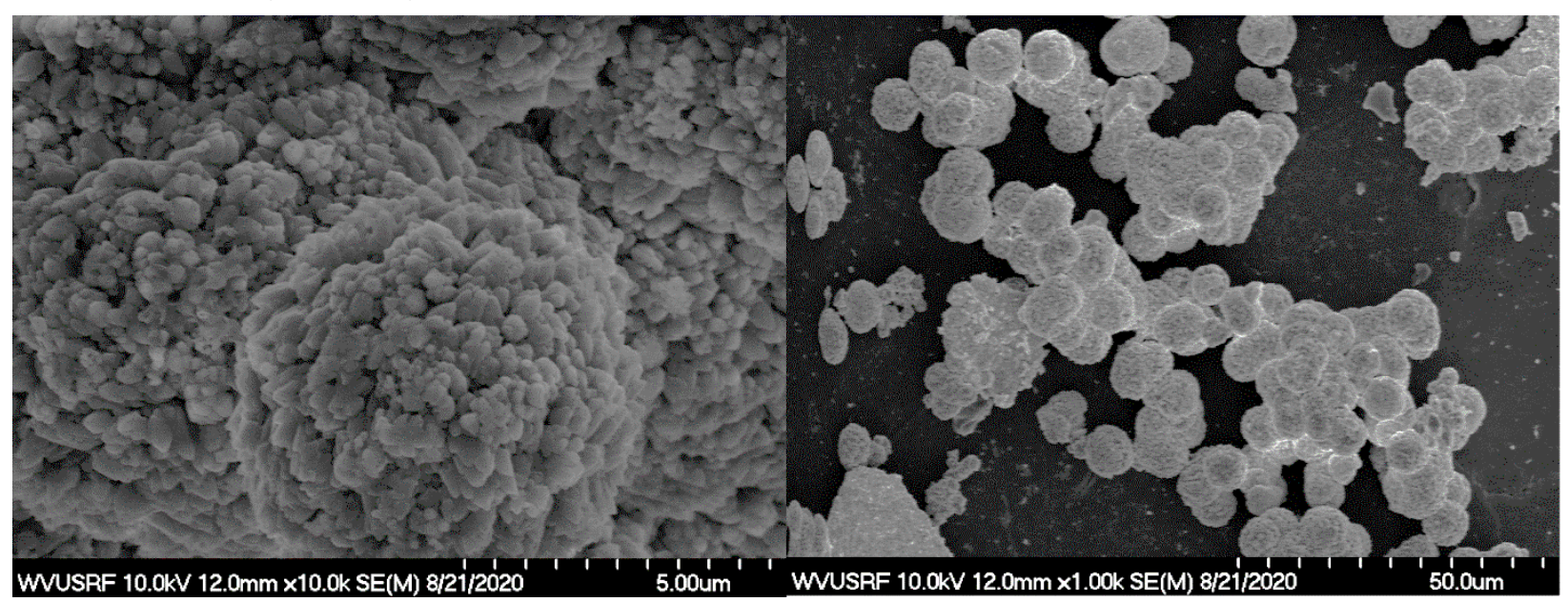

Figure 3.18: SEM imaging of $\mathrm{SrMoO}_{4}$ multi-faceted spheres after hydrothermal processing. 
The XRD peak patterns at $18.8^{\circ}, 27.7^{\circ}, 30^{\circ}, 33.7^{\circ}, 36.8^{\circ}, 38.5^{\circ}, 44.1^{\circ}, 45.4^{\circ}, 47.9^{\circ}, 51.8^{\circ}, 56.3^{\circ}$, $57.5^{\circ}, 61.9^{\circ}, 70^{\circ}, 72.2^{\circ}, 72.6^{\circ}, 76.4^{\circ}, 76.9^{\circ}$, and $79.5^{\circ}$ from sample S3.5.1 were in good agreement with standard $\mathrm{SrMoO}_{4}$ diffraction patterns, confirming that the particles prepared in this study are that of $\mathrm{SrMoO}_{4}$. This XRD pattern is shown below in Figure 3.19.

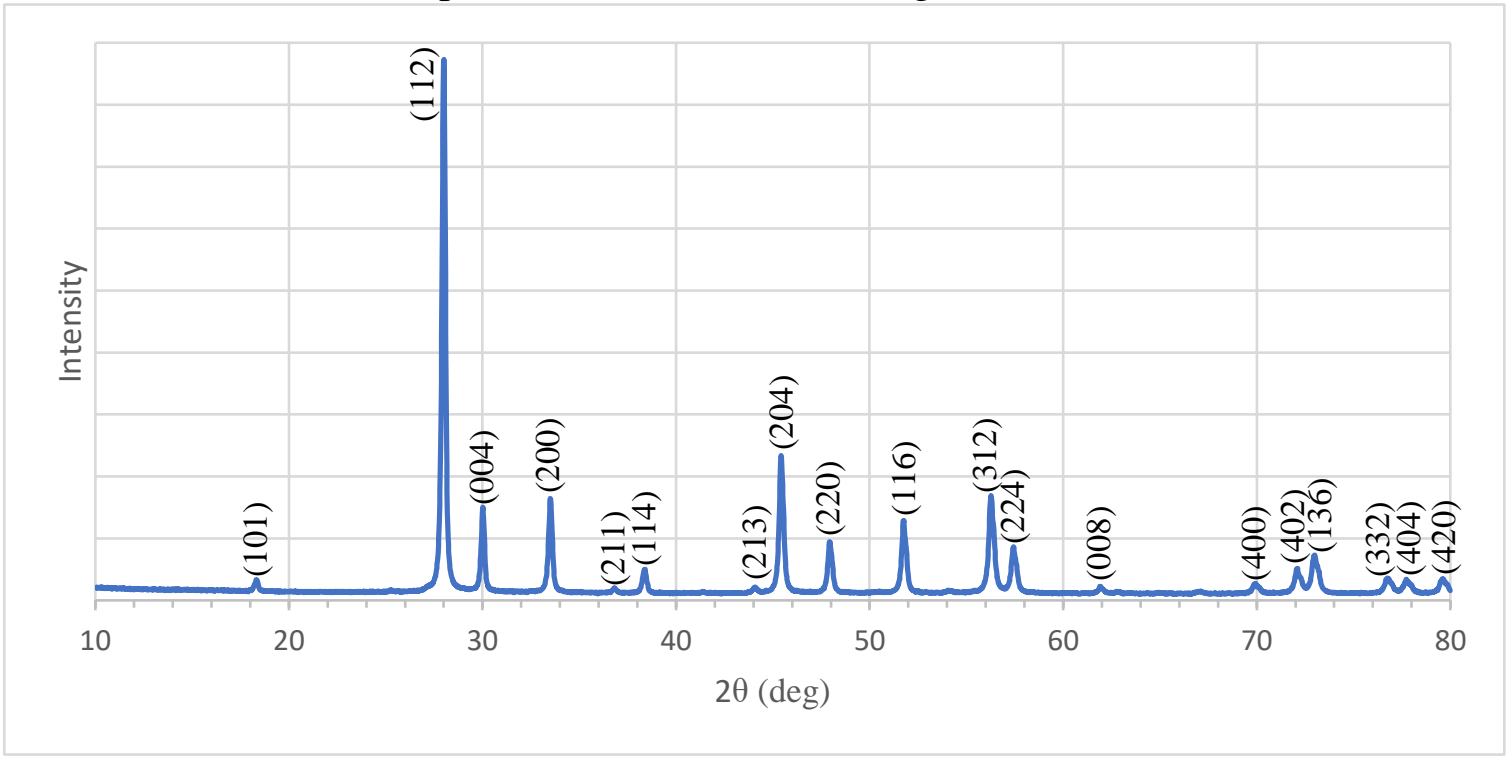

Figure 3.19: XRD pattern of $\mathrm{SrMoO}_{4}$ faceted spheres (S3.5.1).

Since the large microspheres with deep facets and grooves were obtained at the beginning of the synthesis process, no further experiments were conducted. The goal of this synthesis was to test these larger particles against stock nanoscale powders to determine their dielectric differences, not $\mathrm{SrMoO}_{4}$ 's ability to be manipulated into different morphologies.

\subsubsection{Dielectric Comparisons of Shaped vs. Stock Metal Oxides}

A large majority of catalysts used in particle beds are of typical morphologies (spherical, cubic, etc.) of their respective chemistries, thus also having more predictable properties that are standard from particle to particle of uniform sizes. However, a material's dielectric property can alter drastically from crystallographic changes that occur during synthesis. Voids, displacements, particle size, morphology, etc. can change the dielectric properties of the material. Morphological 
changes are the largest dielectric manipulating factor, as particles with incredibly atypical shapes compared to that of spheres and cubes absorb electromagnetic energy differently. This section will cover dielectric differences observed between most of the materials synthesized within this chapter and compare them to stock nanoparticles. The goal is to see if morphologies with thin profiles and sharp edges are greater at absorbing microwave radiation and releasing it in the form of heat than stock particles. Additionally, the particles were not to have dielectric constants that were too high or low, as this would lead to too rapid of heating or no heating at all, respectively. Each powder sample was suspended in paraffin wax at different volumetric percentages to determine their dielectric constants. Logarithmic polynomials were fitted to each powder/paraffin sample. The theoretical Looyenga Mixing Equation shown below was then used to approximate the true dielectric constant of the powder samples. The Looyenga Mixing Equation is an estimation of a material's dielectric properties while suspended in paraffin. All equations for determining dielectric constants have some level of estimating and errors associated with their final values. Adjusting the Looyenga Mixing Equation estimates the dielectric properties of the powder suspended within the paraffin; however, this estimation is the most accurate when the vol\% of the powder is $\geq 5$ vol $\%$. Volumetric percentages higher than $5 \%$ will have higher dielectric constants with less error associated with them (see Table 3.1), but for the purposes of comparison and the ease of testing, all comparisons in this section were between samples tested at $5 \mathrm{vol} \%$.

$$
\varepsilon_{m i x}{ }^{1 / 3}=V_{m} \varepsilon_{m}{ }^{1 / 3}+V_{p} \varepsilon_{p}{ }^{1 / 3}
$$

Table 3.1 lists a comparison of each material's dielectric property between the atypical morphologies of $\mathrm{Fe}_{3} \mathrm{O}_{4}, \mathrm{CaMoO}_{4}$, and $\mathrm{SrMoO}_{4}$ that were synthesized vs. stock, nanoparticle sized samples at volumetric percentages between 5-10\% suspended in paraffin wax. 
Table 3.1: Dielectric Properties of Synthesized Vs. Stock Particles at volumetric percentages between $5-10 \%$ suspended in paraffin wax

\begin{tabular}{|c|c|c|c|c|c|c|}
\hline Sample I.D. & Material & Morphology & Powder Vol\% & Paraffin Vol\% & $\varepsilon^{\prime}$ & $\varepsilon^{\prime \prime} / \varepsilon^{\prime}$ \\
\hline S3.2.7 & $\mathrm{Fe}_{3} \mathrm{O}_{4}$ & Nano-flakes & $5 \%$ & $95 \%$ & 2.144 & 0.0163 \\
\hline S3.2.7 & $\mathrm{Fe}_{3} \mathrm{O}_{4}$ & Nano-flakes & $10 \%$ & $90 \%$ & 8.718 & 0.0405 \\
\hline S3.1.1 & $\mathrm{Fe}_{3} \mathrm{O}_{4}$ & Nano-rods & $5 \%$ & $95 \%$ & 14.880 & 0.0619 \\
\hline S3.1.1 & $\mathrm{Fe}_{3} \mathrm{O}_{4}$ & Nano-rods & $10 \%$ & $90 \%$ & 22.590 & 0.1 \\
\hline S3.3.2 & $\mathrm{Fe}_{3} \mathrm{O}_{4}$ & Micro-octahedrals & $5 \%$ & $95 \%$ & 43.852 & 0.0132 \\
\hline S3.3.3 & $\mathrm{Fe}_{3} \mathrm{O}_{4}$ & Micro-platelets & $5 \%$ & $95 \%$ & 29.319 & 0.0811 \\
\hline N/A & $\mathrm{Fe}_{3} \mathrm{O}_{4}$ & Stock & $5 \%$ & $95 \%$ & 3.371 & 0.0268 \\
\hline S.3.4.1 & $\mathrm{CaMoO}_{4}$ & Clusters/needles & $5 \%$ & $95 \%$ & 7.086 & 0.0196 \\
\hline S.3.4.4 & $\mathrm{CaMoO}_{4}$ & Micro-needles & $5 \%$ & $95 \%$ & 7.464 & 0.0133 \\
\hline S.3.4.5 & $\mathrm{CaMoO}_{4}$ & Micro-clusters & $5 \%$ & $95 \%$ & 1.011 & 0.0147 \\
\hline S.3.4.3 & $\mathrm{CaMoO}_{4}$ & Micro-doughnuts & $5 \%$ & $95 \%$ & 2.817 & 0.0144 \\
\hline N/A & $\mathrm{CaMoO}_{4}$ & Stock & $5 \%$ & $95 \%$ & 3.505 & 0.0104 \\
\hline S3.5.1 & $\mathrm{SrMoO}_{4}$ & Facetted spheres & $5 \%$ & $95 \%$ & 1.526 & 0.0114 \\
\hline N/A & $\mathrm{SrMoO}_{4}$ & Stock & $5 \%$ & $95 \%$ & 0.858 & 0.0118 \\
\hline
\end{tabular}

\subsubsection{1 $\mathrm{Fe}_{3} \mathrm{O}_{4}$ Dielectric Comparisons}

$\mathrm{Fe}_{3} \mathrm{O}_{4}$ particles had noteworthy dielectric divergences between the stock nanoparticles and the shaped particle. The stock particles are the most comparable to S3.2.7 nanoflakes. This was the only example that fell short of the stock sample that was tested, with a dielectric constant of 2.144 compared to 3.371, respectively. All other samples of $\mathrm{Fe}_{3} \mathrm{O}_{4}$ showed average to high dielectric constants with a high variance to one. Micro octahedral $\mathrm{Fe}_{3} \mathrm{O}_{4}(\mathrm{~S} 3.3 .2)$ had the largest dielectric constants at 43.852, followed by microplatelets (S3.3.3), nanarods (S3.1.1), stock nanoparticles, and nanoflakes (S3.2.7), respectively from highest to lowest. As expected, the octahedron and platelet particles were able to absorb more microwave radiation than all samples with nanosized particles. The larger volume for each of these particles allows for more electromagnetic energy to be absorbed compared to the nanosized particles. Yet, when comparing the loss tangents $\left(\varepsilon^{\prime \prime} / \varepsilon^{\prime}\right)$, or 
a materials ability to convert radiation into heat, of each particle, this electromagnetic absorbing capability trend begins to diverge. Although the octahedral particles have the highest ability to absorb radiation, it also has the lowest ability to release that energy as heat, acting more so as an insulator. Comparisons from the highest loss factor to the lowest are as follows: platelets>nanorods $>$ stock $>$ nanoflakes $>$ octahedral. The micron platelets show the highest combined dielectric capabilities with the highest ratio of electromagnetic radiation adsorption and diffusion. Ultimately, a distinction can be made that the alteration of particle's sizes and morphologies of the same chemical makeup can have profound effects on their dielectric capabilities. Larger particles can absorb more radiation while thinner morphologies are better suited at releasing that energy in the form of heat. Smaller particles are able to easily release energy but have lower dielectric constants due to a lack of mass per particle. For optimal particle heating, the volume of each individual particle must be large enough to absorb more electromagnetic radiation yet thin enough to not withhold it.

\subsubsection{2 $\mathrm{CaMoO}_{4}$ and $\mathrm{SrMoO}_{4}$ Dielectric Comparisons}

Similar distinctions were made regarding $\mathrm{CaMoO}_{4}$ samples compared to the trends seen between the $\mathrm{Fe}_{3} \mathrm{O}_{4}$ samples. Although most of these individual particles were of the same size, the same samples, S3.4.1 and S3.4.4, did contain additional phases of $\mathrm{CaMoO}_{4}$ with drastically different structures. Both S3.4.1 and S3.4.4 contained large needle like particles, much greater in size to any other phase recorded among all $\mathrm{CaMoO}_{4}$ samples. Likewise, sample containing these larger needles had larger dielectric constants. The sample with the greatest dielectric constant consisted of only micro-needle microstructures, while the next highest had both needles and small platelet clusters of $\mathrm{CaMoO}_{4}$. Both approximately doubled the dielectric constant of the stock particles, while maintaining the same chemistry. The one sample of $\mathrm{SrMoO}_{4}$ also experienced this trend, as its constant was approximately double as well with the large facetted spheres. Inversely, the 
$\mathrm{CaMoO}_{4}$ samples not containing the large needle-like particles had similar, yet lower dielectric constants to the stock example.

Ultimately, the distinction can be confirmed that changing the particle shape and size both play a roll in determining the average dielectric constant of a material, therefore altering the way that same particle would interact with reagents within a microwave reactor. Some of these alterations evidently increase a catalytic particle's ability to convert electromagnetic radiation into heat, therefore increasing that material's utility within microwave reactor environments since this would inevitably increase the thermal energy a material was able to release at constant power level. In conclusion, the largest versions of each material synthesized $\left(\mathrm{Fe}_{3} \mathrm{O}_{4}, \mathrm{CaMoO}_{4}\right.$, and $\left.\mathrm{SrMoO}_{4}\right)$ displayed greater dielectric constants than their respective stock samples. $\mathrm{Fe}_{3} \mathrm{O}_{4}$ achieve dielectric constants capable of heating very well within a microwave reactor environment, but both $\mathrm{CaMoO}_{4}$ and $\mathrm{SrMoO}_{4}$ did not achieve a high constant. $\mathrm{CaMoO}_{4}$ and $\mathrm{SrMoO}_{4}$ under any microstructural configuration would require a substrate more capable at converting microwave energy into heat to be viable within a reactor environment using microwave irradiation as the main heating source.

\subsection{Conclusions}

Development of new catalysts and reactor designs are ongoing as every manufacturer attempts to streamline their chemical production processes with higher efficiency and lower cost. New reactor designs are beginning to implement MW heating apparatuses over conventional heating methods, as they have greater heating efficiency, flexible design, and reduced capital cost in comparison. MW heating is immediate and is highly effective at converting energy into bulk heating depending on the material being irradiated. The key to MW heating is based on the ability of a material to store and dissipate electromagnetic energy, also known as a material's loss tangent. A loss tangent is the dissipation factor of the sample or how efficiently MW energy is converted into thermal 
energy. It is defined as the ratio of the dielectric loss, or complexed permittivity ( $\varepsilon ")$, to the dielectric constant $\left(\varepsilon^{\prime}\right)$. Materials with high complex permittivities or low dielectric constants heat very well when placed in an electromagnetic field. Dielectric properties of a material are mainly reliant on temperature, MW frequency, and chemical composition of the materials, although the phase and morphology of a material are also defining variables. A particle with a thinner shape would theoretically heat differently when compared to a sphere or cube of similar size as it would have different dielectric properties. Altering the morphology of catalytic particles into atypical shapes was completed through using SDA's and various synthesis methods. Three materials were chosen for shaped synthesis: $\mathrm{Fe}_{3} \mathrm{O}_{4}, \mathrm{CaMoO}_{4}$, and $\mathrm{SrMoO}_{4}$. These materials were produced in various morphologies, such as platelets, octahedrons, nanoflakes, nanorods, doughnuts, as so on. Once the final morphologies of these materials were finalized, the were tested using the transmission line and free space method on an Agilent Technologies N5231A PNA-L Microwave Network Analyzer over a range of 0.1 to $13.5 \mathrm{GHz}$. The particles were added to a paraffin plug between 5-10 vol\%. Each dielectric data set was fitted to a polynomial and averaged to determine the dielectric constant of the paraffin/metal oxide plug. The theoretical Looyenga Mixing Equation was then used to approximate the true dielectric constant of each metal oxide with differing morphologies and compare them the stock particles of the same chemistries. Larger particle sizes of each metal oxide possessed a great dielectric constant than that of stock samples. Particle sizing was determined to be the driving factor for altering a material's immediate dielectric constant, but this was not necessarily true for how they would release that electromagnetic energy in the form of heat. The second determining factor for the creation of ideal microwave absorbing catalytic particles was the final shape of the particle being used. $\mathrm{Fe}_{3} \mathrm{O}_{4}$ micro-platelets showed the highest dielectric constant to loss factor ratio of all materials tested. A particle showed the highest 
dielectric constants when it has higher masses per particle, while thinner morphologies showed higher loss tangents due to their inability to withhold the electromagnetic radiation, instead releasing it quickly as heat. Such observations in how the same materials can possess greater dielectric properties by altering their synthesis processes is paramount to the development of better catalysts more suitable for the ever-growing list of chemical reactions preformed in new microwave reactor designs. Through these small synthesis alterations, well known catalysts previously used with these reactions in conventionally heated reactors may remain thermally viable during these same reactions in new, cost-effective MW reactors. 


\section{Chapter 4: Microwave Irradiation of Decorated Dried PRB Coal Char Catalysts in Dry Reforming Reaction Conditions}

\subsection{Introduction}

Natural gas is a hydrocarbon fuel source used for heating, cooking, and electricity generation, but through the mid-late $20^{\text {th }}$ century, it has seen use as a fuel for vehicles and as a chemical feedstock in the manufacture of plastics and other commercially important organic chemicals. Natural gas is comprised of a naturally occurring hydrocarbon gas mixture consisting mainly of methane and varying amounts of higher alkanes, as well as a small percentage of carbon dioxide, nitrogen, hydrogen sulfide, or helium at times. Methane is the largest component of the hydrocarbon mixture in natural gas. The widespread adoption of natural gas use has made methane one of the most readily available, cost effective hydrocarbon fuel sources. Methane on its own is a relatively efficient fuel source for much of the world due to its relative energy density and wide availability. However, methane can be utilized as a precursor to create other highly profitable, energy dense fuels and chemical compounds such as syngas, ethylene, ethane, methanol, etc. Synthesis of these fuels require the activation or partial activation of methane, cleaving anywhere from one or all its strongly bound hydrogen atoms. Methane's $\mathrm{C}-\mathrm{H}$ bonds are very stable and need high temperatures $\left(>700{ }^{\circ} \mathrm{C}\right)$ to activate. When methane is fully dehydrogenated into $2 \mathrm{H}_{2}+\mathrm{C}(\mathrm{s})$, solid carbon remains and near immediately it begins accumulating in the reaction environment. This occurrence is a common problem with hydrocarbon decomposition reactions, and it commonly refer to as coking. Despite the persistence of coke formation, methane dehydrogenation is very advantageous, since the production of $\mathrm{H}_{2}$ is incredibly valuable as a fuel or as a precursor for further fuel generation. Hydropyrolysis of low-ranking coal (LRC) and methane upgrading 
processes such as the dry reforming of methane (DRM) both use methane for enhanced fuel generation since it has a high hydrogen-to-weight ratio and low cost.

Typical DRM processes react methane in the presences of a light oxidant, $\mathrm{CO}_{2}$. As the methane is decomposition into $2 \mathrm{H}_{2}+\mathrm{C}(\mathrm{s}), \mathrm{CO}_{2}$ reacts with the solid carbon deposits within the reaction environment, $\mathrm{CO}_{2}+\mathrm{C}(\mathrm{s})$, creating two $\mathrm{CO}$ molecules. The use of $\mathrm{CO}_{2}$ is incredibly useful since it can typically be found with methane in natural gas and reacting it into $\mathrm{CO}$ as a fuel precursor makes much better use of the molecule than directly expelling it into the atmosphere. DRM is an effective form of syngas production, and the use of $\mathrm{CO}_{2}$ works to a certain degree for carbon deposition removal, but eventually the system will still coke as the rate of deposition is not equally proportional to the rate of removal. A schematic of the overall DRM reaction network is found below in Figure 4.1.

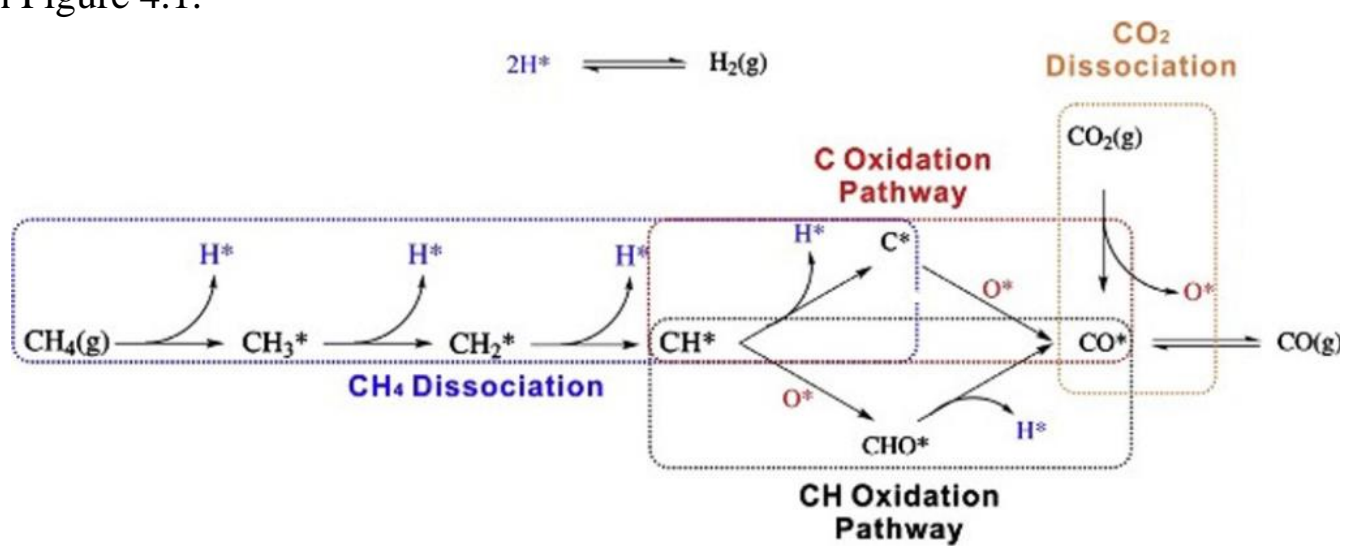

Figure 4.1: A schematic diagram of the reaction network of dry reforming methane (Bian, Das,

Wai, Hongmanorom, \& Kawi, 2017).

Studies have shown that this higher rate of carbon deposition occurs more so the inlet of the reactor vs. the outlet above $850{ }^{\circ} \mathrm{C}$, confirming more carbon was from the $\mathrm{CH}_{4}$ decomposition. Supplementing higher ratios of $\mathrm{CO}_{2}: \mathrm{CH}_{4}$ will assist in this carbon removal, but this normally overcomplicates the DRM process. Increasing $\mathrm{CO}_{2}$ reduces $\mathrm{H}_{2}$ production as $\mathrm{CH}_{4}$ conversion 
drops and will require $\mathrm{CH}_{4}$ separation units to recover unreacted methane down the line, therefore keeping the reactant gases at a 1:1 ratio is optimal.

Methane decomposition is an endothermic reaction with significant energy requirements. Conventional methods of heating reactors for methane reformation operate between $850-950{ }^{\circ} \mathrm{C}$ to invoke sufficient conversion of $\mathrm{CH}_{4}$, as well as $\mathrm{CO}_{2}$ when considering DRM processing. Conventional heating allows both the gas and a heterogeneous catalyst to be at the same temperature. Temperatures above $700{ }^{\circ} \mathrm{C}$ begin to destabilize methane, and the presence of a catalyst allows for some molecules to partially activate into methyl radicals allow its surface. These radicals then desorb back into the gaseous phase and contribute to secondary reactions to create heavier hydrocarbons through carbon coupling. These secondary reactions can lead to the formation of desirable fuels such as ethane, ethylene, etc. However, under normal conditions, these are considered undesirable products that dilute syngas production due to their low selectivity and require separation units down the production line. Normally, the formation of heavier hydrocarbons is typically sought after, but since many of these reactions happen in the gaseous phase, there is no good way of controlling their formation leading to randomized, low selectivities. Higher temperatures help upend this issue by fully dehydrogenating $\mathrm{CH}_{4}$ at the cost of higher energy requirements. Either way, this creates a problem that only creates more cost to the manufacturer using conventional heating methods.

Common catalyst design for methane reforming reactions is centered around the prevention of carbon deposits. Popular anti-coking catalysts are derived from the platinum group metals and row four transition metals $(\mathrm{Fe}, \mathrm{Co}$, and $\mathrm{Ni}$ ). Platinum metals are highly coke resistant, but are not economical for industrial applications, thus lending interest to affordable, earth-abundant transition metals to meet the demand. Nickel is the most popular of these, it has a high affinity for 
methane conversion but unfortunately easily deactivates from coking in its raw form. Ni binds carbon too tightly to be removed by $\mathrm{CO}_{2}$ effectively, effecting the rate of absorption. Ni requires a support with high amounts basic active sites to assist the $\mathrm{CO}_{2}$ adsorption. Basic sites create Lewis acid-base pairs with $\mathrm{CO}_{2}$ since it is slightly acidic and adsorbs more readily to the surface to react alongside the dehydrogenation of $\mathrm{CH}_{4}$ on the active metal sites. Basic supports also aid in the dispersion of $\mathrm{Ni}$ on their surfaces, increasing the number of active sites. Numerous $\mathrm{Ni} / \mathrm{basic}$ supports are quite efficient at negating carbon deposition as the last decade has produced over 700 publications on Ni-based catalysts, but the most used in industry are $\mathrm{Ni} / \mathrm{Al}_{2} \mathrm{O}_{3}$ and $\mathrm{Ni} / \mathrm{SiO}_{2}$. The creation of coking resistant catalysts for hydrocarbon-based reactions has set the groundwork for the next steps in methane reforming innovations.

Currently, all methane-upgrading reactions require substantial amounts of energy due to the endothermic nature of the reaction. Conventional heating is not thermally efficient process, typically needing to create reactionary environments much higher than other methods to match conversion rates (Eryildirim et al., 2021). As expected, this translates to a needlessly higher costly for manufacturers, easily mitigated using other heating methods. Recent interest in alternative heating methods have begun to challenge conventionally designed heating apparatus. Microwave (MW) heating has some of the highest potential the future of reactor designs. MW heating, as the name would suggest, radiates electromagnetic energy into a catalytic reactor bed that contains materials of favorable dielectric properties, predominately materials with high loss tangents. A loss tangent is a material's efficiency to absorb electromagnetic energy and convert it into heat ( $\mathrm{Li}$, H. et al., 2020). Highly dielectric materials produce huge temperature gradients, up to $300{ }^{\circ} \mathrm{C}$ suggested in some literature, between the material surface and the surrounding atmosphere. MW irradiation of these materials is direct and immediate, requiring short reactor start-up times. Ideal 
scenarios involving high temperature gradients of between a material surface and the surrounding atmosphere are with heterogenous catalysts. MW irradiation of dielectrically favorable catalysts promotes reagent adsorption and subsequently an increase in primary surface reactions opposed to secondary reactions in the gaseous phase that would typically coincide with surface level reactions. These reactions occur quick and efficiently on the catalyst surface, while the local temperature of the reactor is much lower than what would typically be required for methane destabilization. As stated, secondary reactions would normally materialize in the gaseous phase as partially reacted methane free radicals couple in the energized atmosphere. However, the surrounding gases are much cooler than the MW irradiated catalyst bed, minimizing secondary gas phase reactions since the medium in which they react do not have the necessary energy in comparison. MW irradiation of catalyst beds provide solutions to typical problems that arise from convention heating methods. MW reactors have the added benefits of less capital, high thermal efficiencies, and more design freedom. Catalysts designed with sufficient loss tangent in mind readily convert the MW energy in heat, translating to massive energy cost savings (Metaxas, A. C., \& Meredith, R. J., 1988). Currently, many common catalysts used in the DRM process do not possess the necessary properties to be used directly in MW irradiated reactors. Specifically, DRM catalysts, such as $\mathrm{Ni} / \mathrm{Al}_{2} \mathrm{O}_{3}$ and $\mathrm{Ni} / \mathrm{SiO}_{2}$, do not heat in a $\mathrm{MW}$ reactor and require an additional, highly dielectric supports to be brought to temperature as was covered in Chapter 3 (Li, L. et al., 2017).

Research into MW-ready catalysts for DRM and like-processes are limited, but the technology continues to gain interests as the demand for cost-effective, locally sourced, sustainable, clean energy rises. Although typically Ni catalysts impregnated on basic substrates are not applicable to DRM processes using microwave irradiation as a heating source, the concept of implanting an active methane reforming particle on a high basicity substrate is candid for DRM catalyst design. 
Ideally, MW catalysts designs would capitalize on this proven design concept, focusing on improving its shortcomings, namely its MW permeability, while maintaining its advantages, high methane conversion, basic site abundance, and a resistivity to coking. The common denominator for each of these catalytic design shortcomings is the high MW permeability of the materials they are comprised of, mainly that of their basic substrates. The acquisition of a highly available, costeffective, substrate that is MW irritable as well as highly basic would resolve the issues with DRM catalysts used in MW reactor environments.

Cost effective catalyst designs are always of highest priority. Many of the most efficient catalysts in the world are often overlooked by many manufacturers strictly due to their high capital costs. Although much of catalyst designing focusses on high reaction efficiency for its respective process, the actual utilization of a design is highly dependent on how that cost is translated to the manufacturer. For decades, new materials have replaced older, costly catalysts through material and manufacturing innovations that have driven down their production expenditures. However, the highest degree of cost minimalization is seen when a common process byproduct or waste material can be converted into a valuable commodity that transforms a manufacturer's waste removal costs into profitable raw materials. Taking this concept into consideration, over the last few decades research into alternative uses for organic matter combustion byproducts has grown in popularity as possible catalytic materials for methane reforming reactions. The combustion of coal for instance results in the release of thermal energy and gasses, as well as solid byproducts in the form of silicious ash and char. Char is a fine carbon component of coal pyrolysis, composed of unreacted carbon with various amounts of siliceous ash. Coal char is typically recycled back into a gasifier or used as a precursor to produce activated carbon among other carbon-based materials. Coal char is considered a low-value byproduct of coal pyrolysis, with little profitable utility. However, recent 
studies of syngas formation and fuel upgrading technologies have discovered that carbon-based materials possess high catalytic performances, especially when used for methane cracking processes (Zhang et al., 2011; Abdelsayed et al., 2019; He et al., 2014). Methane cracking over coal itself has become a popular process known as hydropyrolysis, where the coal and methane react with one another to create higher value fuels such as ethylene, syngas, benzene, and other hydrocarbons (Abdelsayed et al., 2019; Wang et al., 2018; Zhu et al., 2017). Likewise, coal char is a rich source of carbon molecules polymerized in a solid, porous matrix with much of the same attributes as the coal from which it was derive. Several studies have investigated the potential of numerous types of coal char as tar and methane cracking catalyst: many coming to similar conclusions of the char initially cracking methane or upgrading tar initially, but quickly losing much of those catalytic capabilities quickly (Han et al., 2014; Liu et al., 2010; He et al., 2014; Sun et al., 2007; Wei et al., 2011). Nonetheless, research has also suggested carbon-based catalyst supports assist in methane activation while extending the reaction lifetime of the catalyst bed by reducing coking to the active sites of the decorated catalyst (Bermudez, J. M. et al., 2012; Fidalgo, B. et al., 2011; Li, L. et al., 2018; Zhang, J. et al. 2011).

Regarding MWDRM processes, low-rank coal (LRC) char could be an ideal candidate as a catalytic supporting material. LRC char is the solid byproduct of coal pyrolysis using LRC to produce value added fuels and chemicals. Unlike char derived from high caloric coal (high-rank coal), LRC char contains many of the impurities initially trapped in the LRC itself. These impurities impede much of the utilization of the char, as they would need removed first before the char could be used as a precursor to other reactions (activated carbon production). However, LRC char and its impurities have been shown to absorb MW radiation and efficiently convert it into heat, as well as react with methane to produce syngas with trace amounts of ethane and ethylene 
(Abdelsayed et al., 2019). Combining these catalytic characteristics with MW dry reforming could be incredibly advantageous since the LRC coal char reacts with methane alone to produce $\mathrm{H}_{2}$ in turn producing solid carbon that reacts with $\mathrm{CO}_{2}$ to form $2 \mathrm{CO}$. The bifunctionality of $\mathrm{LRC}$ char as a DRM catalyst and a microwavable particle demands further research of the potentiality of it being a high surface area, support for MW heated, methane upgrading reactions. Much of the research are carbon-based catalysts and catalytic supports have been done on biochar, coal, and activated carbons, leaving a gap in understanding the potential of using widely available coal char as a catalytic substrate ( $\mathrm{Li}$, L. et al., 2017, 2018, 2019).

The objectives of this study are as follows: 1) to test new material compositions developed in Chapter 3 for DRM processes using MW irradiation, 2) to observe reactions of these materials comparatively to convention reactor tests, and 3) to observe catalytic differences between three variations of these materials (shaped particles alone, shaped particles mixed with Powder River Basin (PRB) coal char, and PRB coal char coated in these same materials at specific weight percentages). Synthesis of the materials (shaped $\mathrm{Fe}_{3} \mathrm{O}_{4}, \mathrm{CaMoO}_{4}$, and $\mathrm{SrMoO}_{4}$ ) that were mixed with PRB char were covered in Chapter 3. These tests were conducted alongside conventionally heated reactors under the same process conditions to compares the material's catalytic effectiveness between the two reactors for the DRM process. The design of the catalysts is to exploit their loss tangent efficiencies and enhance $\mathrm{CH}_{4} / \mathrm{CO}_{2}$ reactions that occur on their surfaces. Additionally, this research will investigate the capability of reusing PRB coal char as a catalytic supporting material. Precipitation of the metal oxides on the surface of PRB coal char will provide insight on the tertiary surface interactions between PRB coal char, the impregnated catalytic materials and the inlet DRM gasses within an MW environment. The materials were evaluated both pre and post reaction (via MW and conventional heating) using scanning electron microscopy 
(SEM), X-ray diffraction (XRD), thermogravimetric analysis (TGA), temperature programmed desorption (TPD), transmission line and free space dielectric methods, and gas chromatography (GC).

\subsection{Experimental Procedures: $\mathrm{Me}_{\mathrm{x}} \mathrm{O}_{\mathrm{y}} /$ Char Catalyst Preparation \& Testing}

\subsection{1 $\mathrm{CaMoO}_{4} / \mathrm{Char}$}

The catalysts were prepared by incipient wetness impregnation of $\sim 5 \mathrm{wt} \% \mathrm{CaMoO}_{4}$ on the surface of PRB coal char with aqueous solutions of calcium nitrate and ammonium molybdate. A solution of $3 \mathrm{ml}$ of $14 \mathrm{M} \mathrm{NH}_{4} \mathrm{OH}$ and $50 \mathrm{ml}$ of DI was mixed with $0.413 \mathrm{~g}$ of calcium nitrate $\left(\mathrm{Ca}\left(\mathrm{NO}_{3}\right)_{2}\right)$ for 30 minutes (Solution A). A second solution (Solution B) made of $12.5 \mathrm{ml}$ of DI and $0.31 \mathrm{~g}$ of $\left(\mathrm{NH}_{4}\right)_{6} \mathrm{Mo}_{7} \mathrm{O}_{24}$ was stirred vigorously until fully dissolved. Next, $3.201 \mathrm{~g}$ of PRB coal char was added to Solution A and sonicated for 1 hour to thoroughly disperse the char. Solution B was added to Solution A slowly dropwise while stirring at $300 \mathrm{rpm}$ until fully integrated. The solution was then transferred to a $100 \mathrm{ml}$ Teflon-lined autoclave and hydrothermally treated at $160{ }^{\circ} \mathrm{C}$ for 5 hours before being cooled to room temperature. The $5 \mathrm{wt} \% \mathrm{CaMoO}_{4} /$ char was centrifuged and washed with ethanol three times. The precipitate was placed on a hotplate set at $80{ }^{\circ} \mathrm{C}$ for 6 hours to dry in open air.

\subsection{2 $\mathrm{SrMoO}_{4} / \mathrm{Char}$}

The catalysts were prepared by incipient wetness impregnation of $\sim 5$ and $10 \mathrm{wt} \% \mathrm{SrMoO}_{4}$ on the surface of PRB coal char with aqueous solutions of strontium nitrate and ammonium molybdate. A typical preparation of $\sim 5 \mathrm{wt} \% \mathrm{SrMoO}_{4}$ on the surface of char began with filling $40 \mathrm{ml}$ of DI water into a beaker and adjusted to $\sim 6 \mathrm{pH}$ by added dilute nitric acid dropwise (Solution A). Next, $950 \mathrm{mg}$ of PBR coal char was added to the dilute nitrite acid solution and placed in a sonication 
bath for 1 hour. A second solution of $43 \mathrm{mg}$ of $\mathrm{Sr}\left(\mathrm{NO}_{3}\right)_{2}$ was magnetically stirred into $20 \mathrm{ml}$ of DI water until dissolved (Solution B). A third solution of $20 \mathrm{ml}$ of DI water and $34 \mathrm{mg}$ of ammonium molybdate $\left(\left(\mathrm{NH}_{4}\right)_{6} \mathrm{Mo}_{7} \mathrm{O}_{24}\right)$ was stirred vigorously until dissolved (Solution $\left.\mathrm{C}\right)$. Solutions B and C were then combined and stirred for an additional 15 minutes. $5 \mathrm{ml}$ of this solution was then added dropwise to Solution A and stirred at $600 \mathrm{rpm}$ to create the $\sim 5 \mathrm{wt} \% \mathrm{SrMoO} / \mathrm{char}$ particles. Dilute ammonium $\left(\mathrm{NH}_{4} \mathrm{OH}\right)$ was used to adjust the $\mathrm{pH}$ of the solution to 8 . The final solution was added to a $100 \mathrm{ml}$ Teflon-lined autoclave and hydrothermally treated at $80{ }^{\circ} \mathrm{C}$ for 8 hours. The $\mathrm{SrMoO}_{4} /$ char precipitate was centrifuged and washed three times with DI water and EtOH, respectively. The precipitate was placed on a hotplate set at $80^{\circ} \mathrm{C}$ for 6 hours to dry in open air. A typical preparation of $\sim 10 \mathrm{wt} \% \mathrm{SrMoO}_{4}$ on the surface of char would follow the same process yet double the reagent proportions.

\subsection{3 $\mathrm{MoO}_{3} / \mathrm{Char}$}

The $\mathrm{MoO}_{3} /$ char catalyst was prepared by incipient wetness impregnation of $\sim 5 \mathrm{wt} \% \mathrm{MoO}_{3}$ on the surface of PRB coal char with an aqueous solution of ammonium molybdate. A $50 \mathrm{ml}$ solution of DI and $0.139 \mathrm{~g}$ ammonium molybdate $\left(\left(\mathrm{NH}_{4}\right)_{6} \mathrm{Mo}_{7} \mathrm{O}_{24}\right)$ were stirred AT 600 rpm until fully dissolved. The solution was then sonicated with $2.375 \mathrm{~g}$ of PRB coal char for 1 hour to ensure proper dispersion. The solution was then heated to $110{ }^{\circ} \mathrm{C}$ to evaporate the dilute ammonium solution. The char was dried in open air at $110{ }^{\circ} \mathrm{C}$ for an additional 12 hours to ensure all water was removed. The $\mathrm{MoO}_{3} /$ char was then heated in a furnace at $400{ }^{\circ} \mathrm{C}$ for 5 hours under Ar gas.

\subsection{4 $\mathrm{Fe}_{3} \mathrm{O}_{4} / \mathrm{char}$}

The catalysts were prepared by coprecipitation impregnation of $\sim 5 \mathrm{wt} \% \mathrm{Fe}_{3} \mathrm{O}_{4}$ on the surface of PRB coal char with aqueous solutions of ferric chloride and ferrous chloride. A typical preparation of $\sim 5 \mathrm{wt} \% \mathrm{Fe}_{3} \mathrm{O}_{4}$ on the surface of char began with dissolving $90 \mathrm{mg}$ of $\mathrm{FeCl}_{3}$ and $43 \mathrm{mg}$ of $\mathrm{FeCl}_{2}$ 
- $4 \mathrm{H}_{2} \mathrm{O}$ in $100 \mathrm{ml}$ of DI water within a nitrogen atmosphere (Solution A). Next, $950 \mathrm{mg}$ of PBR coal char was added to the iron solution and magnetically stirred for 10 minutes. A second solution of $2.5 \mathrm{~mL}$ of $14.5 \mathrm{M}$ ammonium was diluted in $7.5 \mathrm{ml}$ of DI water (Solution B). Solutions B was slowly injected into the iron/char solution at a rate of $1 \mathrm{~mL} /$ minute and stirred for an additional 15 minutes after being fully added. The $\mathrm{Fe}_{3} \mathrm{O}_{4} /$ char precipitate was centrifuged and washed three times with DI water and $\mathrm{EtOH}$, respectively. The precipitate was placed on a hotplate set at $80{ }^{\circ} \mathrm{C}$ for 12 hours to dry in open air then placed within a vacuum for 6 hours.

\subsubsection{Shaped and Stock $M_{x} O_{y}$ Particles Both Mechanically Mixed with PRB Coal Char and Separate}

A select set of shaped catalysts synthesized in Chapter 3 were mixed mechanically with coal char to determine differences in their MW reactor performances compared to that of the same materials precipitated directly on coal char. An amount of each catalyst was mechanically mixed on a roll mill for 30 minutes with the appropriate weight of coal char. A list of the selected materials used, their respective morphologies, and the weight percentage of the material in respect to the total weight of the sample with char are listed in Table 4.1.

Table 4.1: Shaped and Stock Particles Both Mixed and Unmixed with PRB Coal char

\begin{tabular}{|c|c|c|c|}
\hline Sample ID & Mixing Method & Shape & $\begin{array}{c}\text { Wt\% with PRB } \\
\text { Char }\end{array}$ \\
\hline $\mathrm{CaMoO}_{4} \mathrm{~S} 3.4 .2$ & Mechanical & Platelet Clusters & $5 \mathrm{wt} \%$ \\
\hline $\mathrm{CaMoO}_{4} \mathrm{~S} 3.4 .6$ & Unmixed & Platelets & N/A \\
\hline $\mathrm{SrMoO}_{4} \mathrm{Stock}$ & Mechanical & N/A & $5 \mathrm{wt} \%$ \\
\hline $\mathrm{SrMoO}_{4} \mathrm{~S} 3.5 .1$ & Mechanical & Faceted Spheres & $5 \mathrm{wt} \%$ \\
\hline $\mathrm{SrMoO}_{4} \mathrm{~S} 3.5 .1$ & Mechanical & Faceted Spheres & $10 \mathrm{wt} \%$ \\
\hline $\mathrm{SrMoO}_{4} \mathrm{~S} 3.5 .1$ & Unmixed & Faceted Spheres & N/A \\
\hline $\mathrm{Fe}_{3} \mathrm{O}_{4} \mathrm{~S} 3.1 .1$ & Unmixed & Nanorods & N/A \\
\hline $\mathrm{Fe}_{3} \mathrm{O}_{4} \mathrm{~S} 3.2 .7$ & Unmixed & Nanoflakes & N/A \\
\hline $\mathrm{Fe}_{3} \mathrm{O}_{4} \mathrm{~S} 3.3 .2$ & Mechanical & Microoctahedrons & $5 \mathrm{wt} \%$ \\
\hline
\end{tabular}




\begin{tabular}{|c|c|c|c|}
\hline $\mathrm{Fe}_{3} \mathrm{O}_{4} \mathrm{~S} 3.3 .3$ & Mechanical & Microplatelets & $5 \mathrm{wt} \%$ \\
\hline $\mathrm{Fe}_{3} \mathrm{O}_{4}$ Stock & Mechanical & N/A & $5 \mathrm{wt} \%$ \\
\hline
\end{tabular}

\subsubsection{Microwave Reactor and Gas Chromatography Analysis}

A typical MW reactor experiment would begin where $0.5 \mathrm{~g}$ of a catalyst would be loaded into a quartz tube with an inner diameter of $8 \mathrm{~mm}$. First, quarts wool would be pushed into the center of the quartz tube, followed by the $0.5 \mathrm{~g}$ of catalyst powder, and then pressed into place using another wad of quartz wool to form a packed bed. The catalyst was packed firmly enough so that the bed was static, having no moving particles but not too firm to disallow the flow of gas through it. The loaded quartz tube would be loaded into the *insert name of MW reactor here* where a thermocouple would monitor the temperature inside the bed while the other monitored the temperature exiting the tube. The inlet gas was set at a 200 SCCM of nitrogen, methane, and carbon dioxide with a ratio of $2: 1: 1$, respectively. The reactor was set to hold a temperature of $600{ }^{\circ} \mathrm{C}$ using a $5.85 \mathrm{GHz}$ frequency. While the reaction was occurring, the outlet stream of gasses was analyzed and recorded using gas chromatography. When the reaction concluded, each sample was weighed to determine the amount of catalyst lost to the reaction. Samples not containing coal char underwent TGA analysis to determine if any carbon deposition occurred on their surfaces. These same operating parameters were used when samples were placed with a conventionally heated reactor.

\subsection{Particle Characterizations}

DRM reactions were carried out in $8 \mathrm{~mm}$ inner-diameter (ID) quartz tube reactor. The $200 \mathrm{SCCM}$ feedstock contained 25 vol\% methane, 25 vol\% carbon dioxide, balanced by nitrogen. The custommade MW reactor Lambda MC1330 has an adjustable frequency range from 5.85 to $6.65 \mathrm{GHz}$, 
with a maximum power is $180 \mathrm{~W}$. The MW reactor set-up is described in previous publications by some of the authors of this paper (Baiet al., 2019; Robinson et al., 2020). The reactor configuration is shown in Figure _. $0.5 \mathrm{~g}$ of catalyst was loaded in each experiment. In this study, the MW frequency was set as $5.85 \mathrm{GHz}$ and weight hourly space velocity was $24,000 \mathrm{~mL} / \mathrm{g}$ cat./hr was used. Before initiating feed gas in every experiment, the catalyst was heated in inert environment (nitrogen). MW irradiation was applied throughout the entire reaction period. E-H tuners (not shown in the figure) were adjusted to maintain the reflectance at nearly zero, so the reflected power can be neglected. A sliding short (not shown in the Figure _) was applied to control the phase position of the microwave so that the catalyst bed utilization can be maximized. The temperature at the center of the catalyst bed was measured by an infrared (IR) pyrometer (Raytek MI3 2 M $\mathrm{SF} 1$, resolution $=1.6 \mathrm{~mm}$, spot size $=5 \mathrm{~mm})$. A proportional-integral $(\mathrm{PI})$ controller was used to maintain the reactor at a desired temperature. The outlet gas products were monitored by a 4-

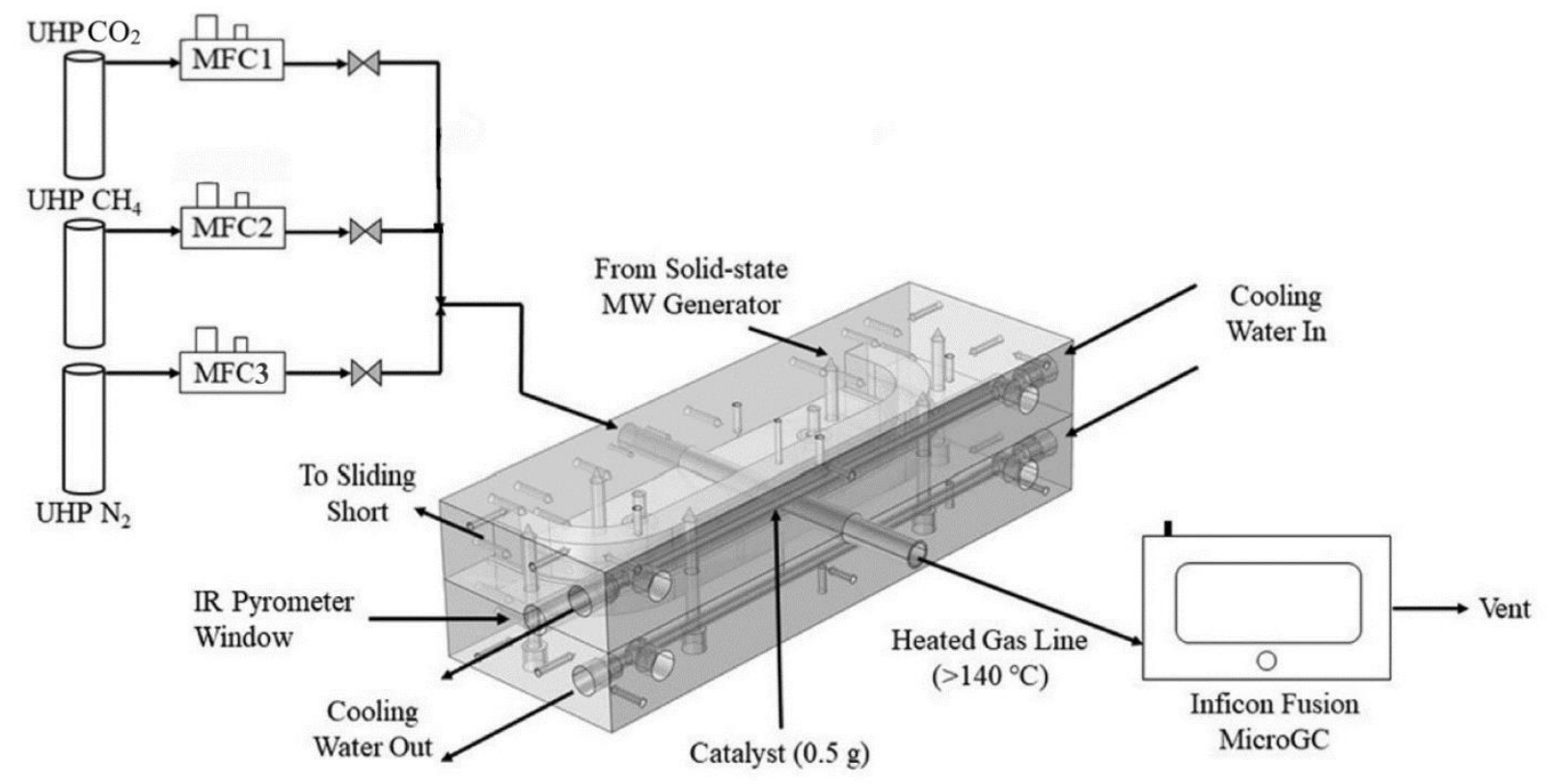

channel Inficon Fusion micro gas chromatography (Micro GC). 
Figure 4.2: Experimental setup for variable-frequency MW reactor (Robinson et al., 2020)

The surface morphology of the post reaction samples was imaged using a field scanning electron microscope (Hitachi S-4700 Scanning Electron Microscope) operating at $45 \mathrm{eV}$ by placing a drop of the suspension solution carefully onto a glass slide on an aluminum stage and letting isopropyl alcohol evaporate slowly in air. Thereafter, each sample was coated with gold (Au) with a Denton Sputter Coater using an Au sputter technique for 140 seconds.

The X-ray diffraction (XRD) patterns for each sample was collected using a PANalytical X'Pert Pro X-ray Diffractometer $\mathrm{CuK} \alpha$ radiation X-ray diffractometer over the range of $20^{\circ} \leq 2 \theta \leq 80^{\circ}$ with $40 \mathrm{~mA}$ and $45 \mathrm{kV}, 0.5 \mathrm{~s}$ step time, and $0.0216^{\circ}$ step size.

Thermogravimetric analysis (TGA) was completed using a TGA-7, PerkinElmer, USA model "Pyris 1." TGA was used on catalysts that were not decorated on coal char to determine levels of coking. The particles were raised to $850{ }^{\circ} \mathrm{C}$ for 10 minutes in a stream of air set at $10 \mathrm{SCCM}$ as their weight was recorded.

$\mathrm{CO}_{2}$ Temperature programmed desorption $\left(\mathrm{CO}_{2}-\mathrm{TPD}\right)$ measurements were completed using a Micromeritics AutoChem 2910 on coal char particles coated with the metal oxide particles to calculate their quantitative $\mathrm{CO}_{2}$ desorption capabilities and the density of basic active sites on their surface. Data was collected using WIN2910 software by raising the temperature of particles from ambient to $850{ }^{\circ} \mathrm{C}$ under $10 \mathrm{~mL} / \mathrm{min}$ flow of air to remove any coking from the $\mathrm{CH}_{4}$ dehydrogenation reaction.

\subsection{Results and Discussion}

Materials synthesized in Chapter $3\left(\mathrm{Fe}_{3} \mathrm{O}_{4}, \mathrm{CaMoO}_{4}, \mathrm{MoO}_{3}\right.$, and $\left.\mathrm{SrMoO}_{4}\right)$ were impregnated on the surface or mechanically mixed with dried PRB coal char to be tested within the two reactor 
systems, one heated using MW radiation and the other using conventional heating. These particles were introduced into the reactor environments and recorded to compare their catalytic influence on $\mathrm{CH}_{4} / \mathrm{CO}_{2}$ dry reforming reactions. The materials were impregnated on the PRB coal char in an attempt to utilize several of char's physical/chemical properties for MWDRM processing: char's porous structure, electromagnetic bulk heating capabilities, and its $\mathrm{CH}_{4} / \mathrm{CO}_{2}$ reforming capabilities. Additionally, impregnating the metal oxide $\left(\mathrm{Me}_{\mathrm{x}} \mathrm{O}_{\mathrm{y}}\right)$ materials on the surface would increase the likelihood of reactions occurring at tertiary points between the char, metal oxides, and gaseous species that would not presumably be seen by simple mechanically mixing. However, specific particles with shaped morphologies synthesized in Chapter 3 and stock nanoparticle powders of the same compositions were also mixed with coal char at various weight percentages for comparisons against those precipitated on char. Some shaped particles, such as $\mathrm{Fe}_{3} \mathrm{O}_{4}$ and $\mathrm{SrMoO}_{4}$, were tested individually in the reactor without a coal char support to isolate their heating and reactionary capabilities, observe any phase changes, as well as determine any coking that could coat the active sites of the materials. TGA was used exclusively for these particles only because any materials containing coal char would simply react with oxygen in the airstream used at 850 ${ }^{\circ} \mathrm{C}$, skewing coking results as the char eroded.

\subsubsection{MWDRM Processing Shaped $\mathrm{Me}_{\mathrm{x}} \mathrm{O}_{\mathrm{y}}$ Catalysts vs. Stock Materials}

Initial reactor testing was completed on four shaped particles: $\mathrm{CaMoO}_{4}$ micron platelets (S3.4.6), $\mathrm{SrMoO}_{4}$ faceted spheres (S3.5.1), and $\mathrm{Fe}_{3} \mathrm{O}_{4}$ nanoflakes and nanorods S3.2.7 and S3.3.1, respectively). These four samples had desirable, thin morphologies (for their respective chemistries) produced in the previous chapter were tested first to serve as a baseline for how these materials would behave within MWDRM reactor conditions. Testing these materials helped define answers that would not have been possible with most of the other materials produced, since all of 
them were either precipitated or mixed with coal char. Coal char being the dominant chemistry for future catalyst testing, weighing $90-95 \%$ of total sample weights, would skew or hide much of the interactions between the reagent gasses and the metal oxide particles within the samples. MW reactor testing on these materials was completed to answer the following:

- Do these metal oxides reform methane and carbon dioxide under MW radiation?

- Is there a difference in reactant conversion rates when using conventional vs. MW reactors?

- Does the shape of the particles effect the DRM reaction of that catalyst?

- Does coking form on the material surfaces and if so, how much?

- How does MWDRM processing effect the morphology of shaped particles?

- How well do these materials absorb MW radiation and disperse that energy as heat?

Answers to these questions were pivotal in understanding the true nature of the solid-gas interactions between the materials synthesized and the inlet gasses before the introduction of coal char within the packed catalyst bed.

A typical reactor set up started with packing 0.5 grams of each catalyst within an $8 \mathrm{~mm}$ ID quartz tube between two wads of quartz wool. The material would then be tested in both a conventional and MW reactor at a local temperature of $600{ }^{\circ} \mathrm{C}$ with $200 \mathrm{SCCM}$ of nitrogen, methane and carbon dioxide flowing through the bed at a 2:1:1 ratio, respectively. As anticipated, none of the catalysts resulted methane reforming or coupling of any kind within the conventionally heated reactor, all having $\sim 0 \%$ conversion of methane and carbon dioxide. Figure 4.3 depicts the gas chromatography (GC) results of $\mathrm{SrMoO}_{4}$ conventionally heated in a dry reforming environment at $600{ }^{\circ} \mathrm{C}$. 


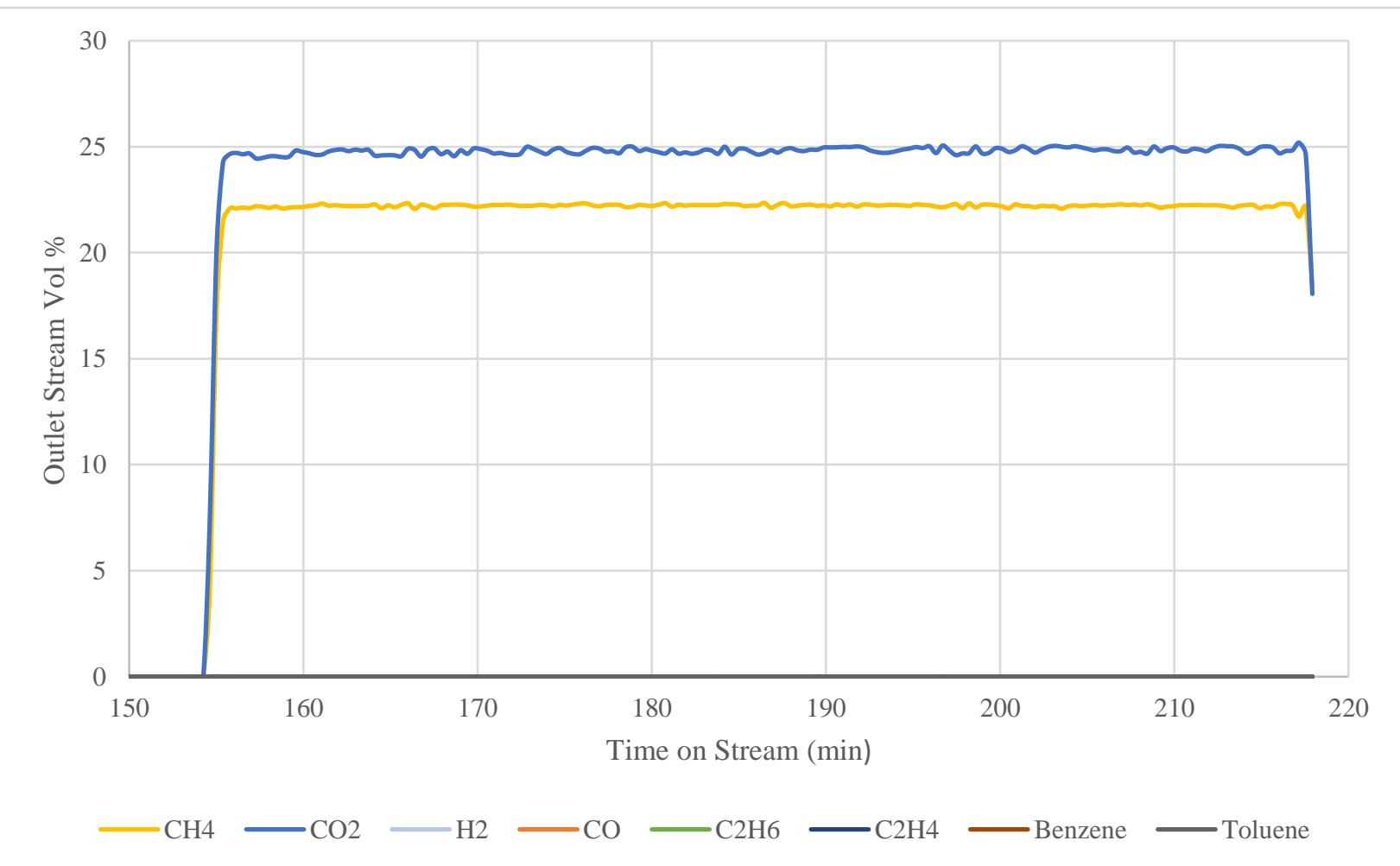

Figure 4.3: Volumetric percentages of outlet stream gasses over $\mathrm{SrMoO}_{4}$ catalyst within a DRM environment conventionally heated at $600^{\circ} \mathrm{C}$.

The GC result of Figure 4.3 are consistent with all materials tested in the conventional reactor environment. No catalytic candidate place in the conventionally heated reactor was able to reform $\mathrm{CH}_{4}$ or $\mathrm{CO}_{2}$. At $600{ }^{\circ} \mathrm{C}$, the temperature is not high enough to destabilize methane molecules, and each catalyst was not able to lower the activation energy requirement for methane to be activated. Inversely, all but $\mathrm{CaMoO}_{4}$ showed some level of syngas reforming within the MW reactor at the same local temperature of $600{ }^{\circ} \mathrm{C}$. $\mathrm{CaMoO}_{4}$ was not able to reach this temperature, and $\mathrm{SrMoO}_{4}$ was only able to come to $300{ }^{\circ} \mathrm{C}$ for initial testing. The samples $\mathrm{Fe}_{3} \mathrm{O}_{4}$ ( $\mathrm{S} 3.3 .1$ and S3.2.7), however, were able to reach the $600{ }^{\circ} \mathrm{C}$ local temperature. Figure 4.4 shows $\mathrm{CH}_{4}$ and $\mathrm{CO}_{2}$ conversion rates for samples S3.3.1 (nanorods) and S3.2.7 (nanoflakes). 


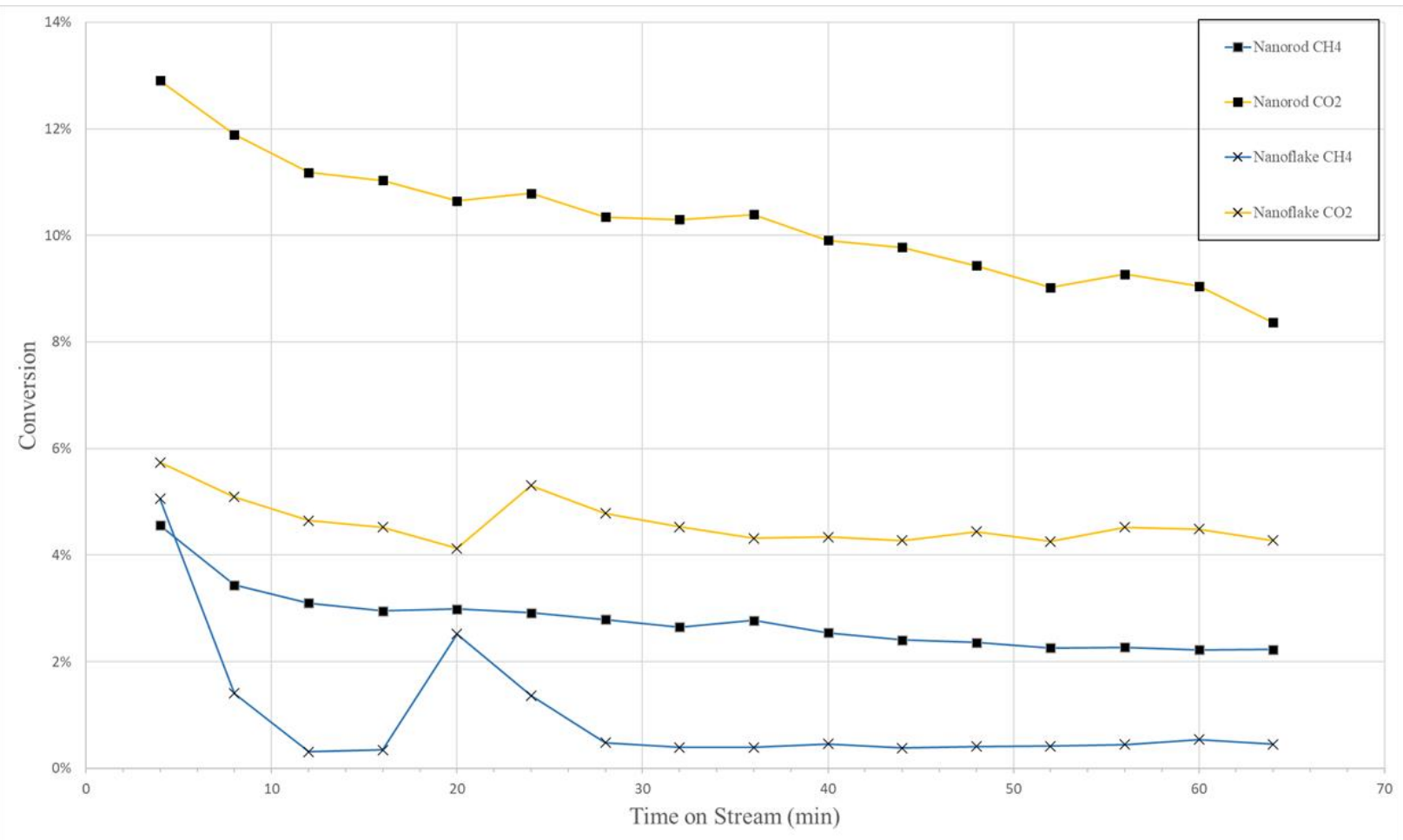

Figure 4.4: $\mathrm{Fe}_{3} \mathrm{O}_{4}$ samples $\mathrm{S} 3.1 .1$ and $\mathrm{S} 3.2 .7 \mathrm{CH}_{4}$ and $\mathrm{CO}_{2}$ conversion rates at $600{ }^{\circ} \mathrm{C}$ within a

5.85 GHz MW reactor under dry reforming conditions

At $600{ }^{\circ} \mathrm{C}$ in the MW reactor, both morphologies of $\mathrm{Fe}_{3} \mathrm{O}_{4}$ were able to reform $\mathrm{CH}_{4}$ and $\mathrm{CO}_{2}$ in some capacity, although S3.1.1 showed higher, more stable rates of conversion. S3.2.7's conversions of $\mathrm{CH}_{4}$ and $\mathrm{CO}_{2}$ peaked initially at $5.1 \%$ and $5.7 \%$, respectively before rapidly falling 8 minutes into the test. $\mathrm{CO}_{2}$ conversion remained steady for much of the reaction, although only trace amount of $\mathrm{CO}$ gas was detected by the $\mathrm{GC}$ unit. $\mathrm{CO}_{2}$ 's conversion rate was assumed to mainly be contributed the polarizing effects of the oxygen and iron atoms of $\mathrm{Fe}_{3} \mathrm{O}_{4}$ like that of $\mathrm{CaO}$ and $\mathrm{SrO}$ molecules theorized in literature (Yang, H. et al., 2006). Although $\mathrm{CH}_{4}$ presented low conversion rates, the reaction products did not record any trace amounts of $\mathrm{H}_{2}$ gas, instead showing the $100 \%$ selectivity for ethane, albeit with an average yield of $<0.02 \%$. Additionally, S3.1.1 also had limited reforming of $\mathrm{CO}_{2}$, only showing brief, negligible instances of $\mathrm{CO}$ in stream. Inversely, S3.1.1 only showed limited initial coupling of ethane for the first 8 minute of the reaction, instead 
showing low levels $\mathrm{CH}_{4}$ and $\mathrm{CO}_{2}$ reforming into syngas for the entirety of the time on stream as seen in Figure 4.5.

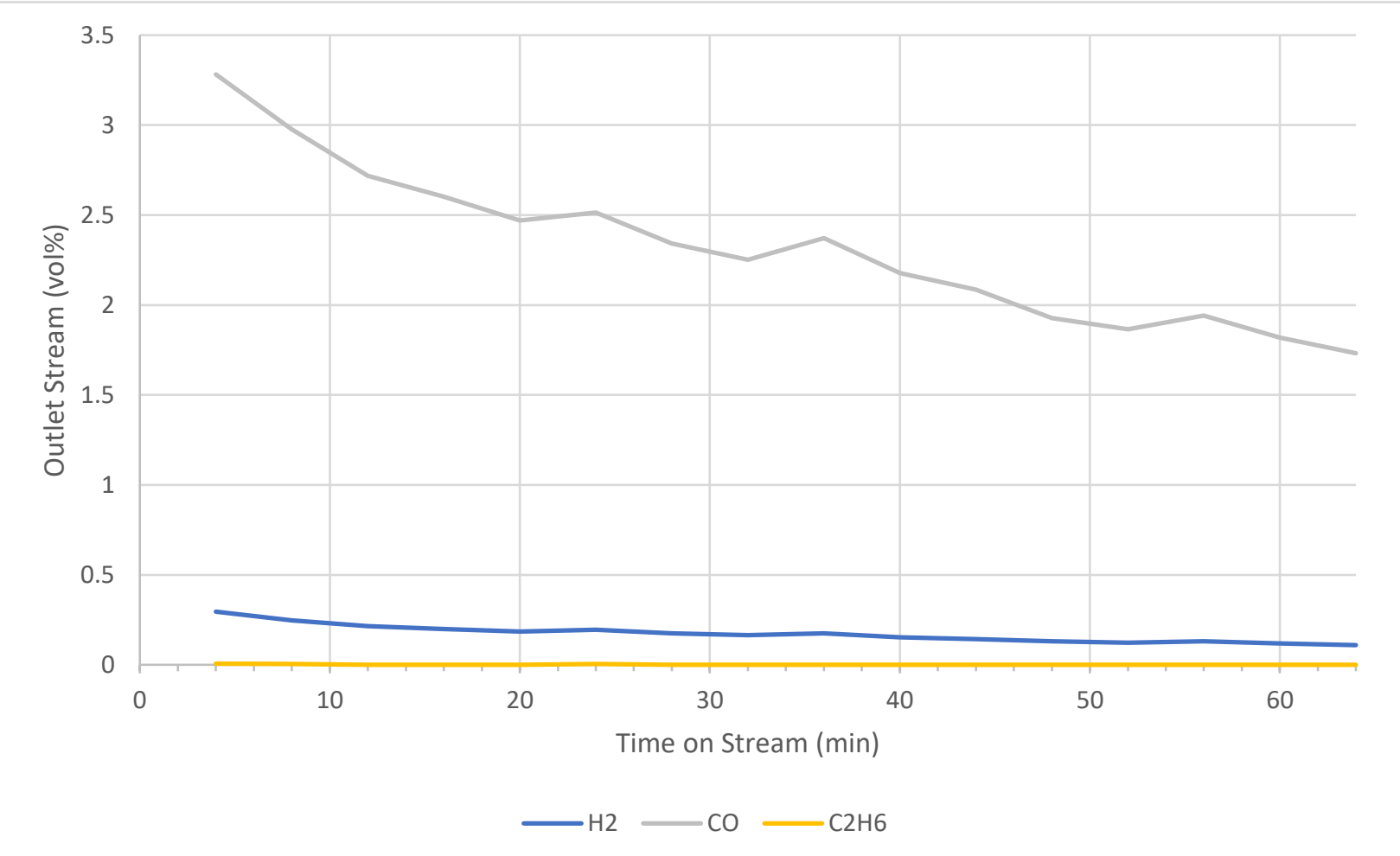

Figure 4.5: Sample S3.1.1 outlet stream vol\% of $\mathrm{H}_{2}, \mathrm{CO}$, and $\mathrm{C}_{2} \mathrm{H}_{6}$ at $600{ }^{\circ} \mathrm{C}$ within a $5.85 \mathrm{GHz}$ MW reactor under dry reforming conditions 
Once removed from the reactor, both samples were recharacterized using XRD and SEM to determine any changes in the material's chemistries and/or morphologies, respectively. Comparisons of samples S3.1.1 and S3.2.7's XRD patterns and SEM images pre/post-reaction are displayed below in Figures 4.6 and 4.7, respectively.

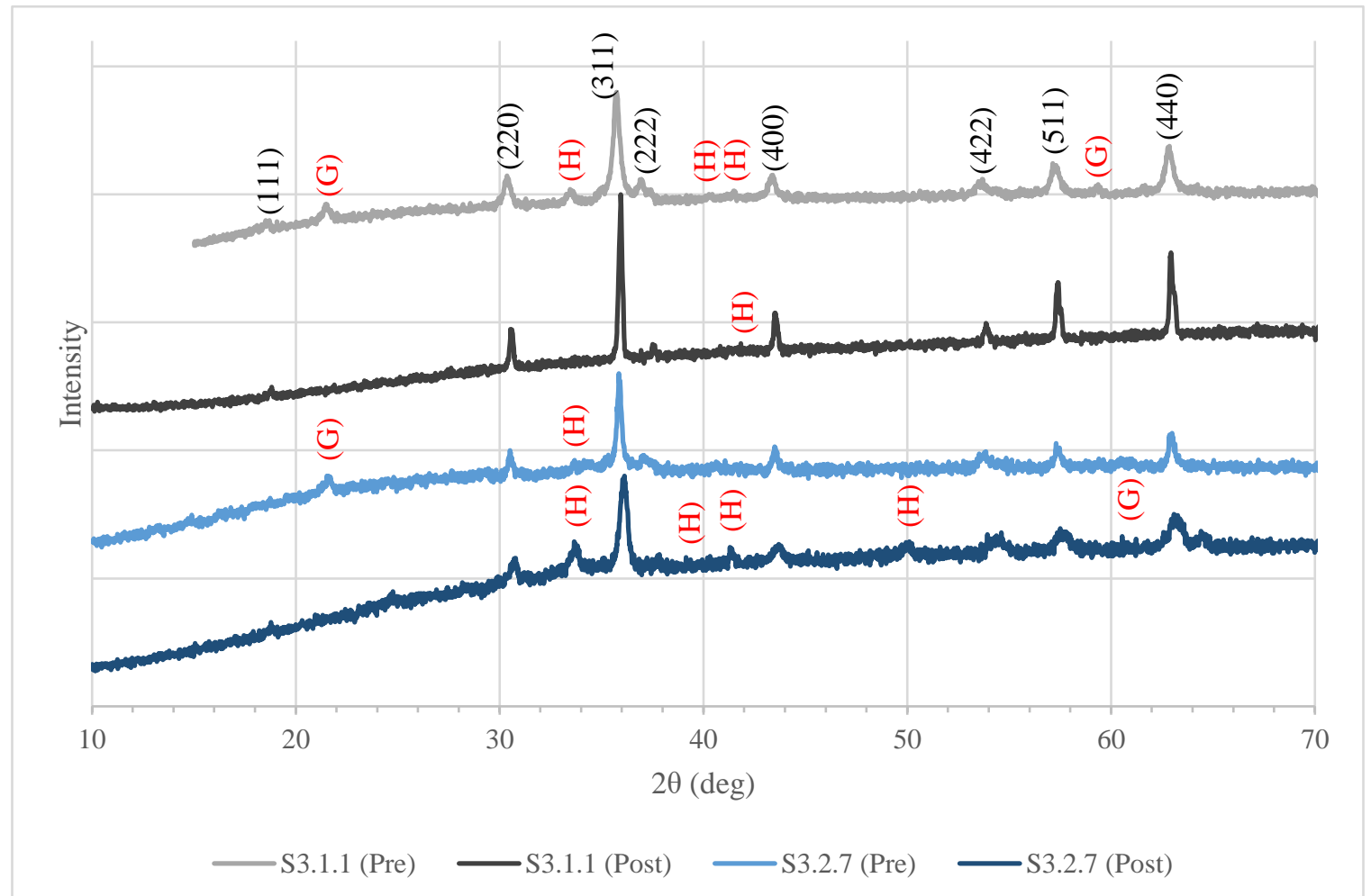

Figure 4.6: XRD patterns of $\mathrm{Fe}_{3} \mathrm{O}_{4}$ sample S3.1.1 and S3.2.7 both pre and post MWDRM reaction. 


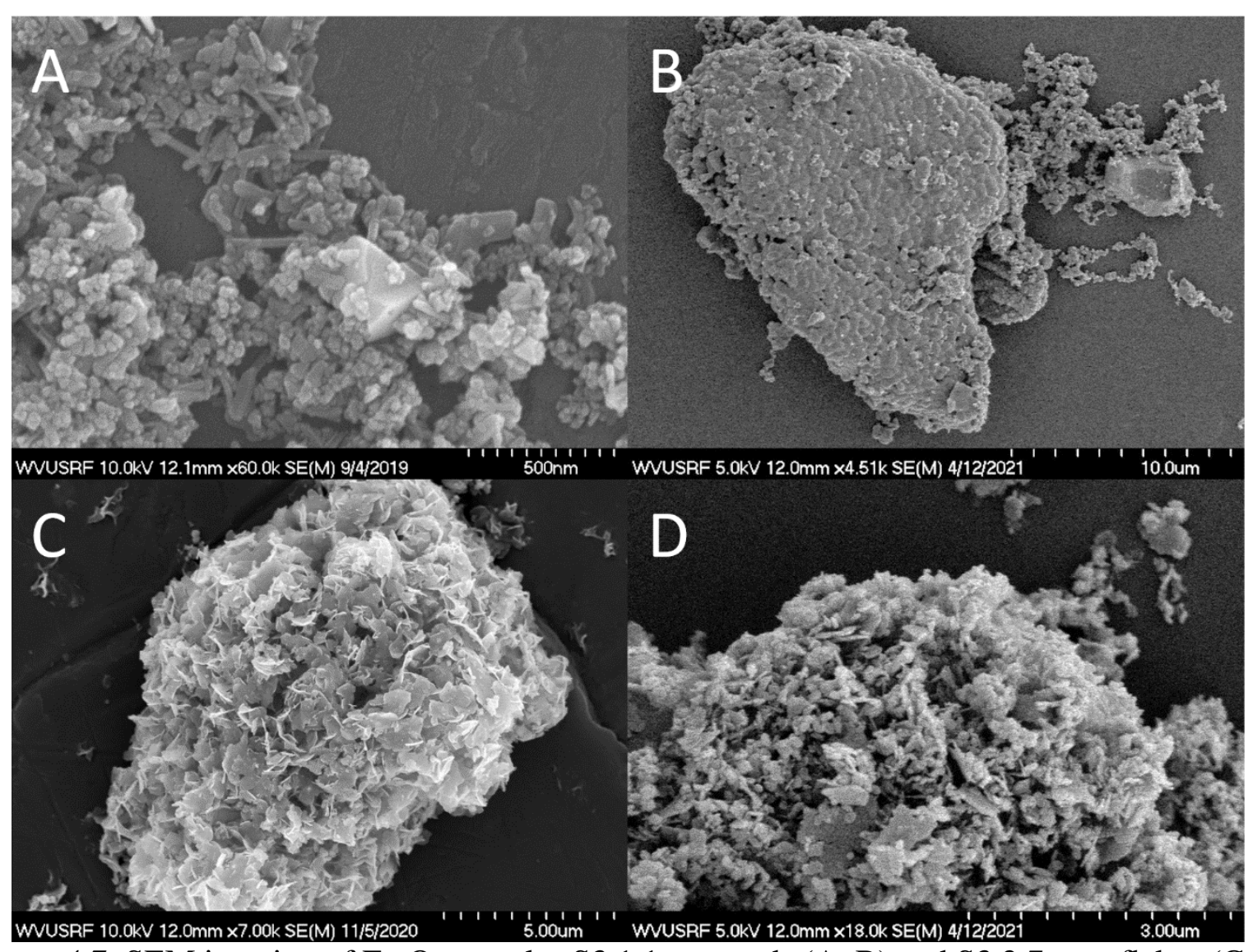

Figure 4.7: SEM imaging of $\mathrm{Fe}_{3} \mathrm{O}_{4}$ samples $\mathrm{S} 3.1 .1$ nanorods $(\mathrm{A}, \mathrm{B})$ and $\mathrm{S} 3.2 .7$ nanoflakes (C, D)

both pre (left) and post (right) MWDRM processing.

Post reaction XRD analysis displays several small changes in the chemistry of each sample. S3.1.1 nanorods experienced beneficially changes after exiting the MWDRM process, overall having a reduction in the trace goethite and hematite impurities, labeled $(\mathrm{G})$ and $(\mathrm{H})$, respectively. The nanorods also presented sharper, higher peak intensities suggesting this sample was further oxidized into $\mathrm{Fe}_{3} \mathrm{O}_{4}$. These improvements were possibly due to the reduction in atypical morphologies after the reaction, opting for the smaller, spherical morphology of $\mathrm{Fe}_{3} \mathrm{O}_{4}$ as seen in Figure 4.5. $\mathrm{CO}_{2}$ within the reactor environment possibly assisted in the alteration of the trace iron hydroxides and hematite remaining, completely oxidizing them into $\mathrm{Fe}_{3} \mathrm{O}_{4}$. Inversely, S3.2.7 experienced the exact opposite reaction, where the peaks broadened, and new peaks formed post 
MWDRM processing. It is less likely that new molecules were formed from the DRM reaction, instead the degradation most likely exposed other materials laden in the original $\mathrm{Fe}_{3} \mathrm{O}_{4}$ nanoflakes. Also, another theory explaining the addition of peaks and peak broadening could have occurred from the $\mathrm{Fe}_{3} \mathrm{O}_{4}$ nanoflakes being incredibly unstable, degrading into other morphologies/chemical compounds with the introduction of reforming gasses and the high heat of the particles themselves.

Before and after imaging of S3.1.1 nanorods showed alterations within the particle's morphologies, as the nanorods are no longer present post-DRM reaction. The particles experienced significant rounding and agglomerating into irregular, round particle with bumpy surfaces as well as the persistent presence of small, sphere-like particles. It was inferred that morphological change was a result from the higher rate of $\mathrm{CH}_{4}$ and $\mathrm{CO}_{2}$ reforming occurring on the surface. Furthermore, this theory was considered plausible when compared against morphological changes seen in S3.2.7 nanoflakes, which somewhat maintained its plate/flake-like structures coinciding with no real reforming and very limited carbon coupling during its time-on-stream (TOS). However, this low reforming may have also occurred/exacerbated with the additional impurities present after the reaction. Finally, TGA analysis on both $\mathrm{Fe}_{3} \mathrm{O}_{4}$ samples did not suggest any significant amounts of coking along their surfaces, both only losing $>0.01 \mathrm{mg}$ of weight after exposure to $10 \mathrm{SCCM}$ of air at $850^{\circ} \mathrm{C}$ to burn off any carbon deposits. After three TGA trials, each graph relatively flat and inconclusive to suggest any significant carbon deposits on the surface of the particles. Overall, the S3.2.7 nanoflakes degraded, had altered morphologies, did not reform, and sustained chemical changes from the DRM processing, ending their candidacy as MWDRM catalysts for the remainder of testing. S3.1.1 nanorods were able to reform in some capacity, so the concept of using $\mathrm{Fe}_{3} \mathrm{O}_{4}$ as a MWDRM catalyst was further explored. 
Unlike $\mathrm{Fe}_{3} \mathrm{O}_{4}$, both calcium and strontium molybdate did not heat as well within the MW reactor. Calcium molybdate would not heat in any capacity, while strontium molybdate could only reach approximately $300{ }^{\circ} \mathrm{C}$, neither of which lead to any significant reforming of methane whatsoever. Since $\mathrm{SrMoO}_{4}$ had previous success with carbon coupling and could slightly heat in the MW reactor, it was then mixed with silicon carbine ( $\mathrm{SiC})$ to raise the catalyst bed temperature and have the local temperature of the system be at the set $600{ }^{\circ} \mathrm{C}$. $\mathrm{SiC}$ has a dielectric constant between 6.5-10, so it heats relatively well within a MW environment without coming to temperature too quickly like that of $\mathrm{TiO}_{2}$, which has a constant of $\sim 80$. Sample S3.5.1 from Chapter $3\left(\mathrm{SrMoO}_{4}\right.$ multi-facetted spheres) was mechanically mixed with $\mathrm{SiC}$ at a 1:1 weight ratio (Sample I.D. S3.5.1). SiC was chosen because it heats well in the MW reactor but has no known catalytic properties with $\mathrm{CH}_{4}$ or $\mathrm{CO}_{2}$. This powder was treated under the same reactionary conditions as the $\mathrm{Fe}_{3} \mathrm{O}_{4}$ particles, heating $0.5 \mathrm{~g}$ of the powder within the $\mathrm{MW}$ reactor at a local temperature of $600{ }^{\circ} \mathrm{C}$ with $200 \mathrm{SCCM}$ of nitrogen, methane and carbon dioxide flowing through the bed at a 2:1:1 ratio, respectively. Thereafter, the spent powder was collected and weighed to determine if any significant weight had been added or lost during the DRM 
reaction. GC analysis of the MWDRM reaction with S3.5.1 particles demonstrated the reforming and coupling potential of $\mathrm{SrMoO}_{4}$ as an individual catalytic particle, yielding sufficient conversion rates of $\mathrm{CH}_{4}$ and $\mathrm{CO}_{2}$ alike seen in Figure 4.8 below.

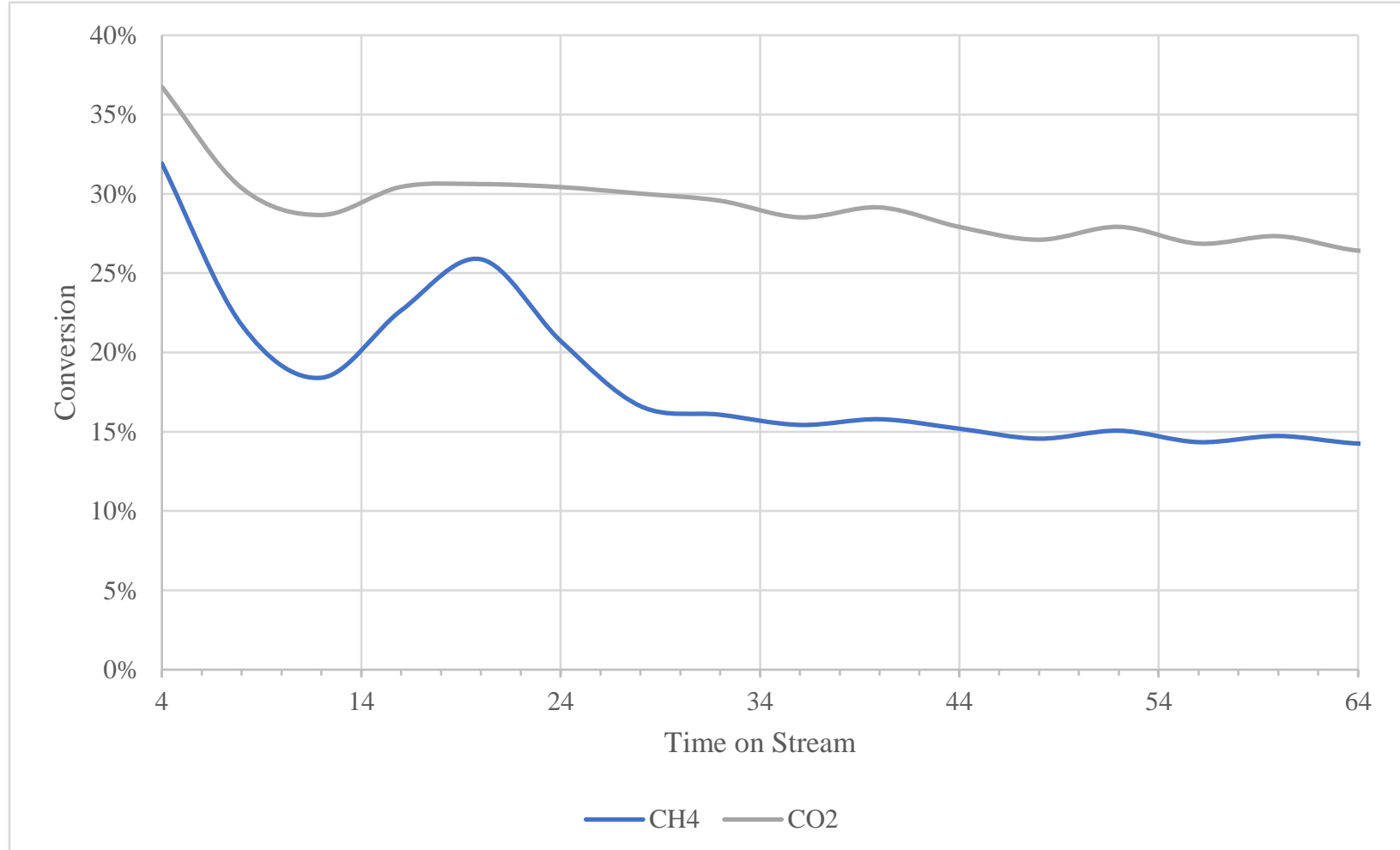

Figure 4.8: $\mathrm{SrMoO}_{4} / \mathrm{SiC}$ sample $\mathrm{S} 3.5 .1 \mathrm{CH}_{4}$ and $\mathrm{CO}_{2}$ conversion rates at $600{ }^{\circ} \mathrm{C}$ within a 5.85 GHz MW reactor under dry reforming conditions.

The conversion of both $\mathrm{CH}_{4}$ and $\mathrm{CO}_{2}$ mainly produced syngas in the outlet stream like that of $\mathrm{Fe}_{3} \mathrm{O}_{4}$ sample S3.5.1, although carbon coupling was also observed in small amounts. Ethane and ethylene formation were small, only accounting for a combined average of $0.08 \%$ of the product gasses outlet volumetric flowrate. However, unlike $\mathrm{H}_{2}$ and $\mathrm{CO}$ formation rates, $\mathrm{C}_{2} \mathrm{H}_{6}$ and $\mathrm{C}_{2} \mathrm{H}_{4}$ remained very consistent through the entirety of the reaction as seen below in Figure 4.9. 


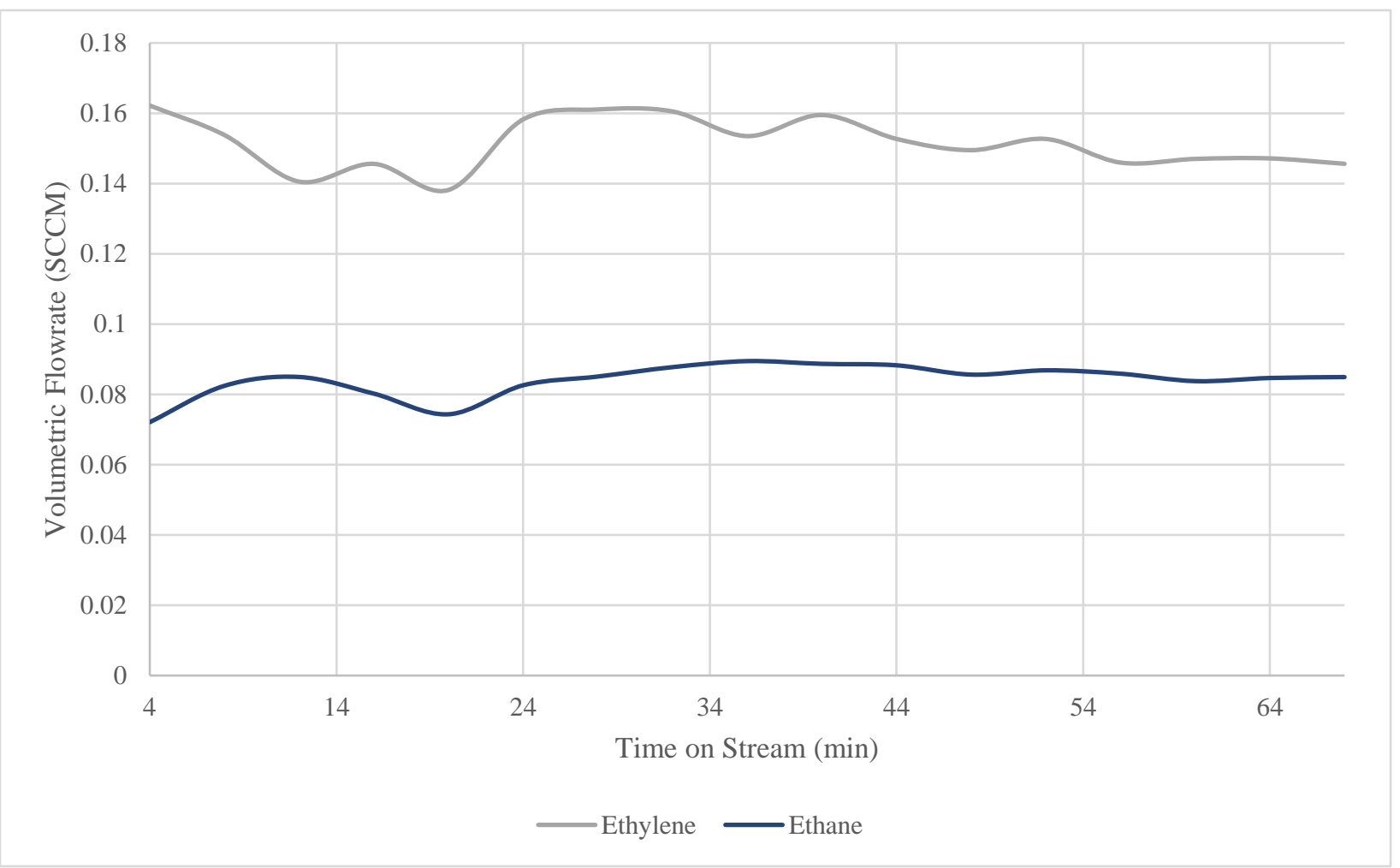

Figure 4.9: Ethylene and ethane volumetric flowrates in the MW reactor outlet stream using $\mathrm{SrMoO}_{4} / \mathrm{SiC}(\mathrm{S} 3.5 .1)$ as a catalyst.

The $\mathrm{SrMoO}_{4} / \mathrm{SiC}$ particles maintained consistent conversion rates of both reactant gases, gradually losing their reforming/coupling abilities as the reaction continued. This would normally be attributed to the active sites slowly coking during the reaction. However, when these particles were tested in the TGA afterwards, no significant changes in weight were seen after three separate exposures to $10 \mathrm{SCCM}$ of air at $850{ }^{\circ} \mathrm{C}$ to burn off any carbon deposits (the same of which was seen with $\mathrm{Fe}_{3} \mathrm{O}_{4}$ particles). Additionally, the weight the bulk particle bed remained unchanged from when it was initially loaded into the quartz tube, eliminating suspicions of catalyst loss being the reason for the reduction in conversion. Catalytic losses through the reaction were mainly attributed to phase alterations in the $\mathrm{SrMoO}_{4}$ 's microstructure, reducing number of exposed active sites, and slowly waning the particles' reaction rates. SEM imaging of the post-reaction 
$\mathrm{SrMoO}_{4} / \mathrm{SiC}$ particles confirms the changes suspected after reviewing the GC data, depicting particle coarsening and reductions in exposed surface areas as seen in Figure 4.10.

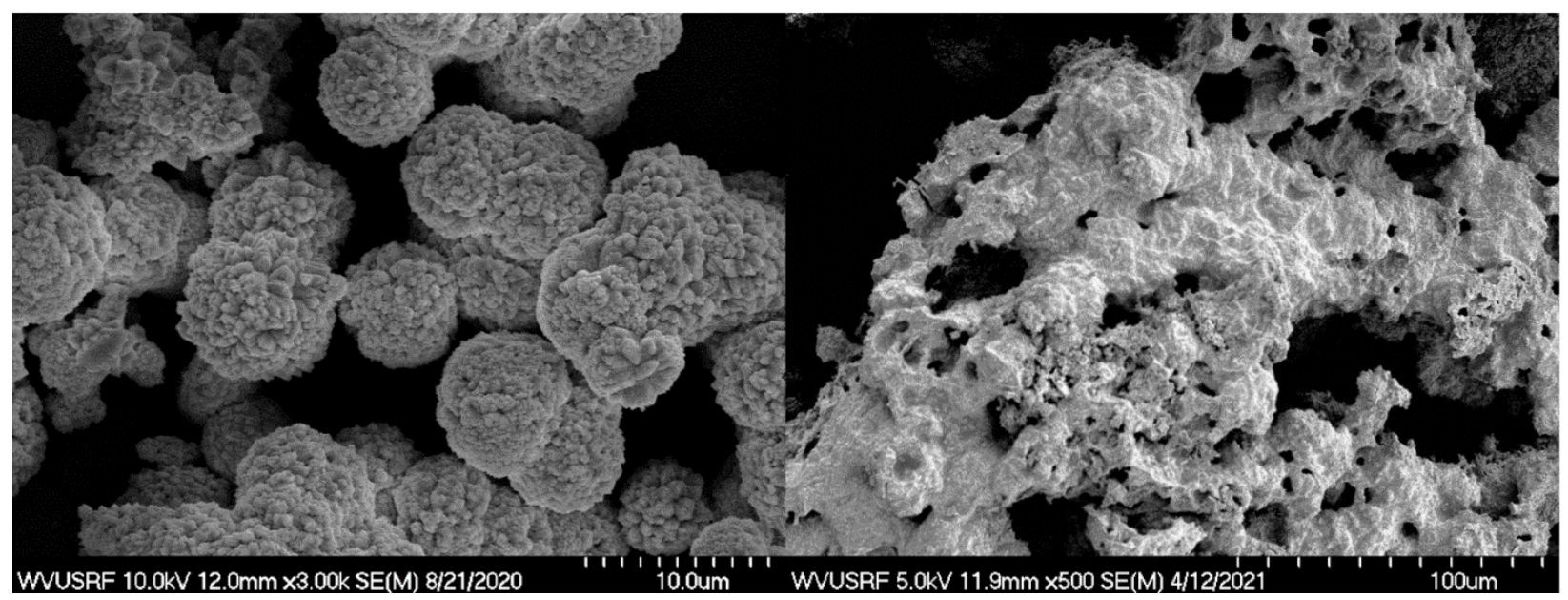

Figure 4.10: SEM imaging of $\mathrm{SrMoO}_{4} / \mathrm{SiC}$ sample S3.5.1 pre (left) and post (right) MWDRM processing.

The microstructure of the $\mathrm{SrMoO}_{4}$ facetted spheres proved to be unstable, as were lost during the MWDRM reaction testing. Much of the individual microstructures instead broke down into much smaller, solid, spheroid-like particles with smooth surfaces or seemed to mold themselves around the $\mathrm{SiC}$ in some cases. The coarsening naturally depleted many of the active sites needed to for the DRM reaction to maintain a consistent reaction rate. However, it can be noted that even with the drastic coarsening seen in the $\mathrm{SrMoO}_{4}$, the ability for carbon coupling to occur was not altered significantly through the duration of the reaction, instead maintaining a steady, albeit low, reaction rate. $\mathrm{SrMoO}_{4}$ demonstrated an innate ability to activate methane molecules without fully dehydrogenating them, followed by limited carbon coupling between the methyl radicals thereafter regardless of what phase the $\mathrm{SrMoO}_{4}$ was in at a given time. $\mathrm{SrMoO}_{4}$ 's initial reactionary duality of reforming and carbon coupling was notably advantageous for the development of value-added fuels, requiring additional testing alongside $\mathrm{Fe}_{3} \mathrm{O}_{4}$ for the majority of this Chapter to help develop 
a greater scope of the material's potential as a fuel synthesizing catalyst during MWDRM reactions.

\subsubsection{MWDRM Processing with PRB Coal Char}

Conventionally heated and MW induced reactor testing was completed on materials both synthesized in various morphologies form Chapter 3 compared against stock nanoparticles of these same materials mixed at varying weight percentages with dried PRB coal char. Additionally, these same materials were precipitated on the surface of the coal char to observe their interactions with the coal char itself, and discover any tertiary interactions between the coal char, metal oxides, and gasses that could occur on the surface. All samples were compared against the catalytic properties of PRB coal char alone within a DRM environment in both the MW and conventional reactors. PRB coal char was used as a baseline comparison for $\mathrm{CH}_{4} / \mathrm{CO}_{2}$ conversion rates and produces formed from the DRM reaction. The coal char was first tested within a conventionally heated reactor at $600{ }^{\circ} \mathrm{C}$ in a $200 \mathrm{SCCM}$ stream of nitrogen, methane and carbon dioxide flowing through the bed at a 2:1:1 ratio, respectively. Similarly, to the testing of $\mathrm{Fe}_{3} \mathrm{O}_{4}$ and $\mathrm{SrMoO}_{4}$ alone, the conventionally heated reactor is not at the appropriate temperature to destabilize the $\mathrm{C}-\mathrm{H}$ or $\mathrm{C}=\mathrm{O}$ bonds present in $\mathrm{CH}_{4}$ or $\mathrm{CO}_{2}$, respectively. Methane itself does not fully begin to decompose until about temperatures of $\sim 700{ }^{\circ} \mathrm{C}$. This trend will remain constant through the remainder of this study, as no material combination had any noteworthy reforming or coupling of any kind within the conventionally heated reactor. A temperature of $600{ }^{\circ} \mathrm{C}$ was not chosen for optimal catalytic conditions, but instead to highlight the effectiveness of MW heating on the same materials at equal localized temperature, as well as, demonstrating that reforming is possible at lower temperatures with MW heating where it is not with conventional heating methods. Figure 4.11 below features 
the MW reforming capabilities of PRB coal char at $600{ }^{\circ} \mathrm{C}$ and $700{ }^{\circ} \mathrm{C}$, showing the conversion of methane and carbon dioxide.

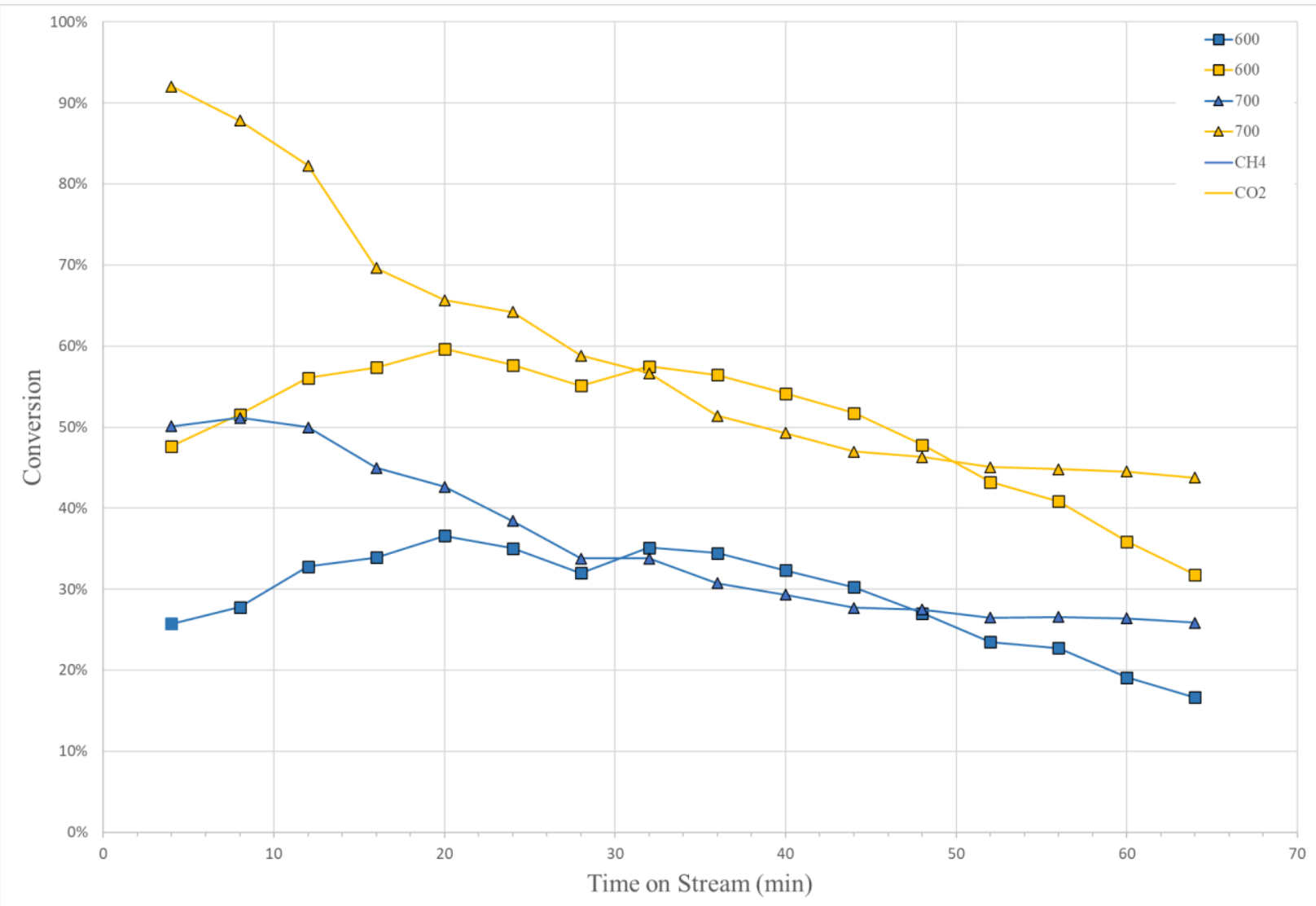

Figure 4.11: Conversion rates of $\mathrm{CH}_{4}$ and $\mathrm{CO}_{2}$ over $\mathrm{PRB}$ coal char within a $5.85 \mathrm{GHz} \mathrm{MW}$ reactor at 600 and $700{ }^{\circ} \mathrm{C}$.

PRB coal char alone is capable of reforming $\mathrm{CH}_{4}$ and $\mathrm{CO}_{2}$, both reaching their peaks of $36.6 \%$ and $59.7 \%$ conversions, respectively, after the first 20 minutes of exposure at $600{ }^{\circ} \mathrm{C}$. The $700{ }^{\circ} \mathrm{C}$ sample however met its peaks of reforming $\mathrm{CH}_{4}$ and $\mathrm{CO}_{2}$ immediately at approximately $50 \%$ and $91 \%$, respectfully. Thereafter, the conversion rates gradually fell, mirroring one another in both scenarios, as the microstructure undergoes changes and some of the catalyst is consumed by the reaction itself. The $700{ }^{\circ} \mathrm{C}$ sample conversion rates fell significantly faster than the $600{ }^{\circ} \mathrm{C}$ sample, but this was attribute to the rapid erosion of carbon within the char, also removing valuable active sites in the process. The coal char had the highest volumetric flowrate yields of $\mathrm{H}_{2}$ and $\mathrm{CO}$ in the 
outlet stream, directly mirror the conversion levels of $\mathrm{CH}_{4}$ and $\mathrm{CO}_{2}$, respectively. However, trace amounts of ethylene and ethane were also present, only rising as the reaction continued. The outlet stream volume percentages of the product gases are seen below in Figures 4.12 and 4.13.

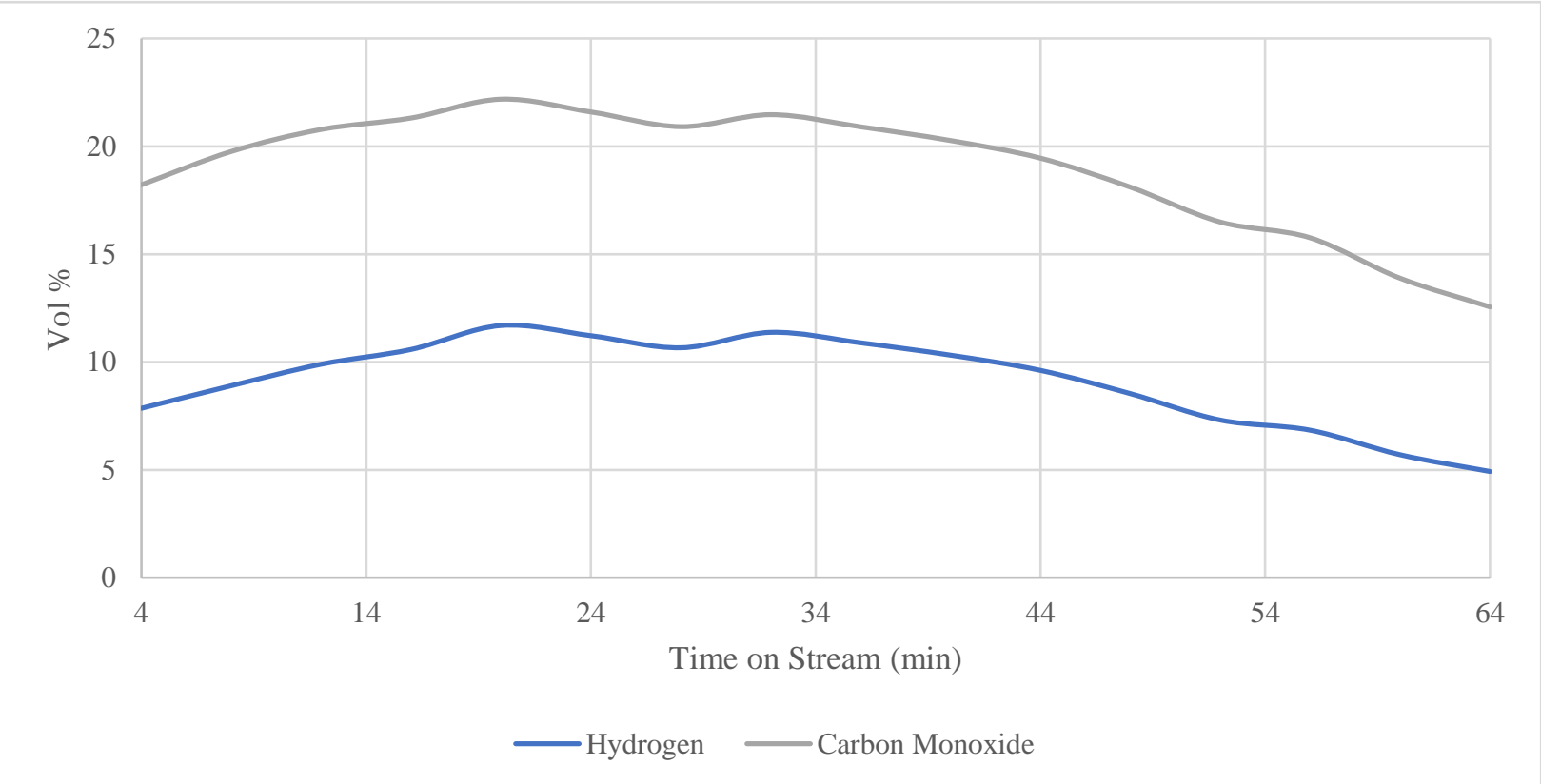

Figure 4.12: Vol\% of $\mathrm{H}_{2}$ and $\mathrm{CO}$ over PRB coal char within a $5.85 \mathrm{GHz} \mathrm{MW}$ reactor at $600{ }^{\circ} \mathrm{C}$.

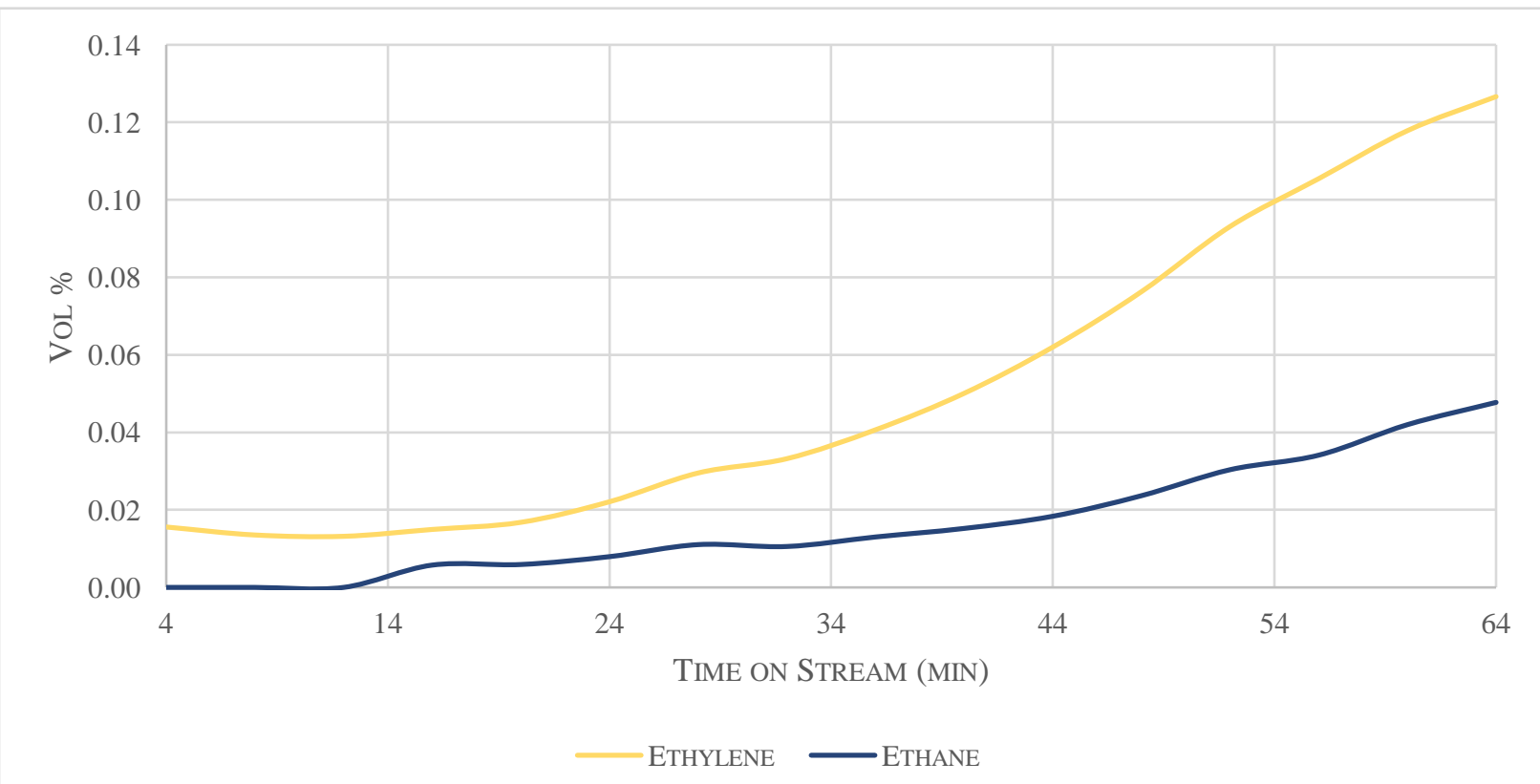

Figure 4.13: Vol\% of $\mathrm{C}_{2} \mathrm{H}_{6}$ and $\mathrm{C}_{2} \mathrm{H}_{4}$ over $\mathrm{PRB}$ coal char within a 5.85 $\mathrm{GHz} \mathrm{MW}$ reactor at 600 ${ }^{\circ} \mathrm{C}$. 
Naturally, coal char is a carbon dense material. This being true, $\mathrm{CO}_{2}$ reacts with this carbon along with the deposited carbon from the methane dehydrogenation reaction, reducing the mass of the catalyst bed. In turn, this weight reduction pared with the coarsening of the coal char particles themselves reduces the amount of available active sites, therefore dropping the reaction rate. Initially, the coal char is small with a flake-like appearance, containing only a few large, solid particles around the matrix. After MWDRM processing, the smaller particles are lesser in number and likely contributing to much of the reforming of the $\mathrm{CH}_{4}$ and $\mathrm{CO}_{2}$, also resulting in the catalyst bed mass reduction. Solid, stable particles are left behind from the reaction that are much larger than the coal char particles preceding the MWDRM processing. It is believed the large particle are contributing to the bulk heating of the bed, but not chemically with the DRM reaction since they remain present here and through all MW reactor tests when combined with metal oxide particles. The PRB coal char pre/post MWDRM processing are seen below in Figure 4.14.

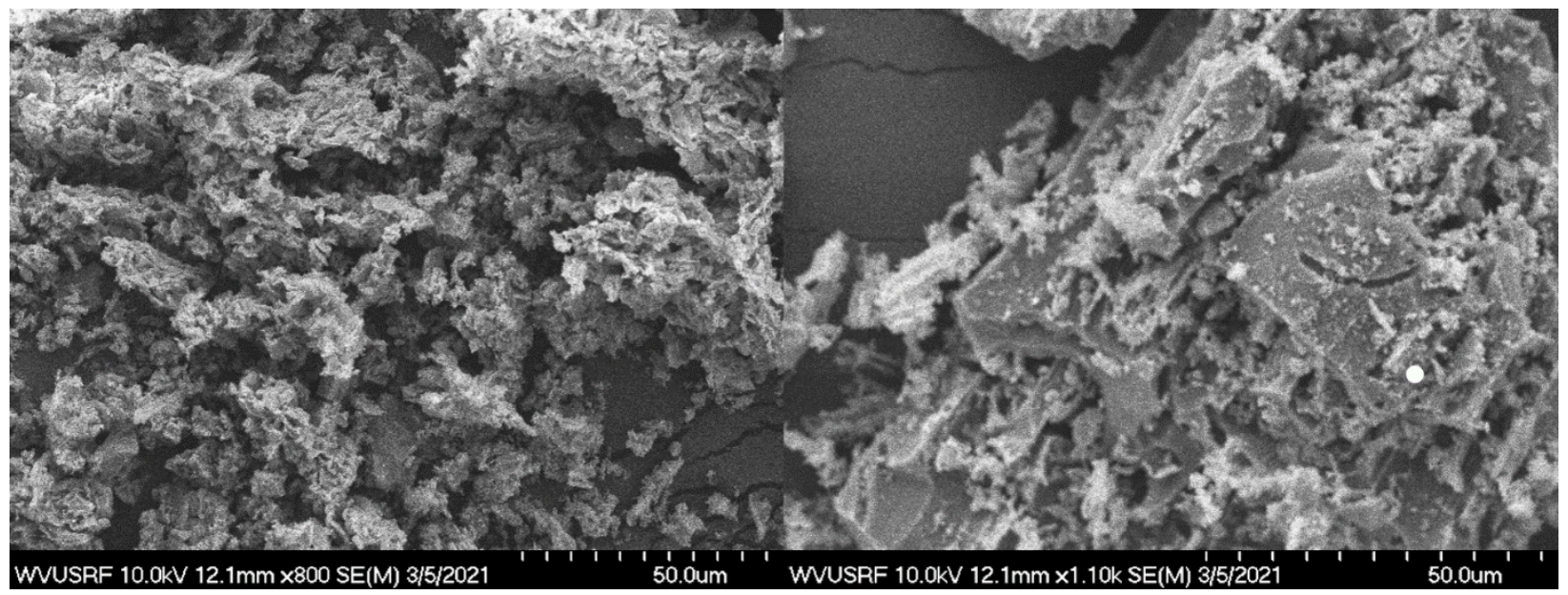
Figure 4.14: SEM imaging of PRB coal char pre (left) and post (right) MWDRM processing at 
Post-reaction, the PRB coal char bed experienced a $45.5 \%$ loss in total mass. A similar loss was recorded after reacting char with only $\mathrm{CO}_{2}$ and the same reactor condition, with a catalyst mass loss of $48 \%$. The $\mathrm{CO}_{2}$ conversion rates between a $1: 1$ ratio of $\mathrm{CH}_{4}: \mathrm{CO}_{2}$ and pure $\mathrm{CO}_{2}$ stream within the MW reactor are shown below in Figure 4.15.

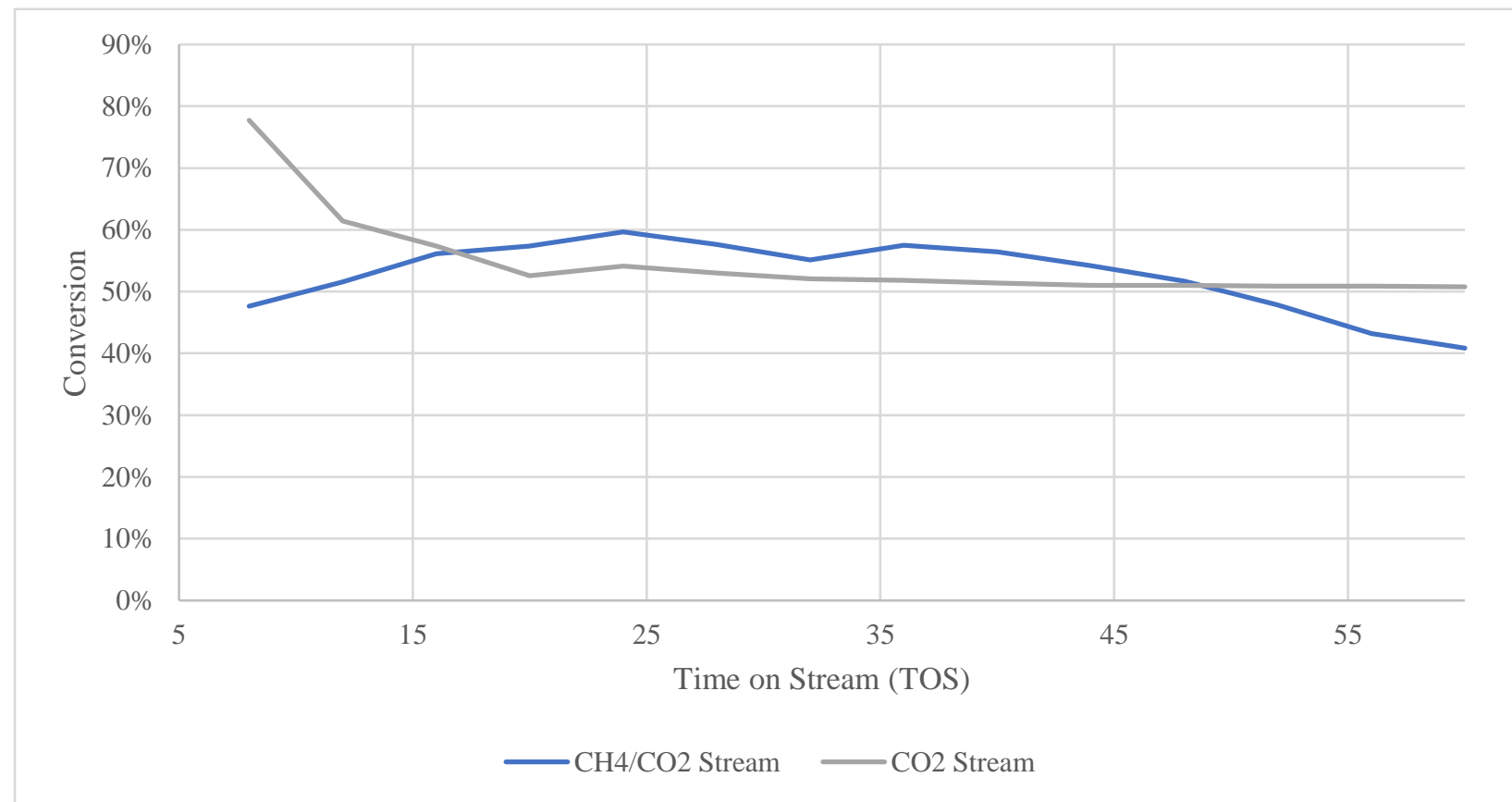

Figure 4.15: Conversion rates of $\mathrm{CO}_{2}$ with altered inlet gas streams over PRB coal char within a $5.85 \mathrm{GHz} \mathrm{MW}$ reactor at $600{ }^{\circ} \mathrm{C}$.

Both cases maintain similar $\mathrm{CO}_{2}$ conversion rates, however the coal char sample in the $\mathrm{CO}_{2}$ only stream started with a higher conversion rate and was able to stabilize just above a $50 \%$ for most of the time on stream. The sample reacting in only $\mathrm{CO}_{2}$ also lost slightly more mass than the sample in the DRM conditions, however, the small discrepancy may have been caused by slight sample loss between the two samples upon removal from the quartz tube and wool post-reaction. Although, the $2.5 \%$ weight loss difference was mainly considered to be due to the presence of methane in the former reaction, mitigating carbon loss from the coal char bed as the $\mathrm{CO}_{2}$ reacted with deposited carbon on its surface. 
PRB coal char was chosen as an ideal catalytic substrate for its clear electromagnetic bulk heating properties, however, initial MWDRM testing also suggested that char possesses $\mathrm{CO}_{2}$ adsorption characteristics after displaying high conversion rates. The amount of $\mathrm{CO}_{2}$ chemisorbed at room temperature was determined to be $104.28 \mu \mathrm{mol} \mathrm{CO} /$ gram char catalyst. The $\mathrm{CO}_{2}$-TPD patterns were obtained as shown in Figure 4.16.

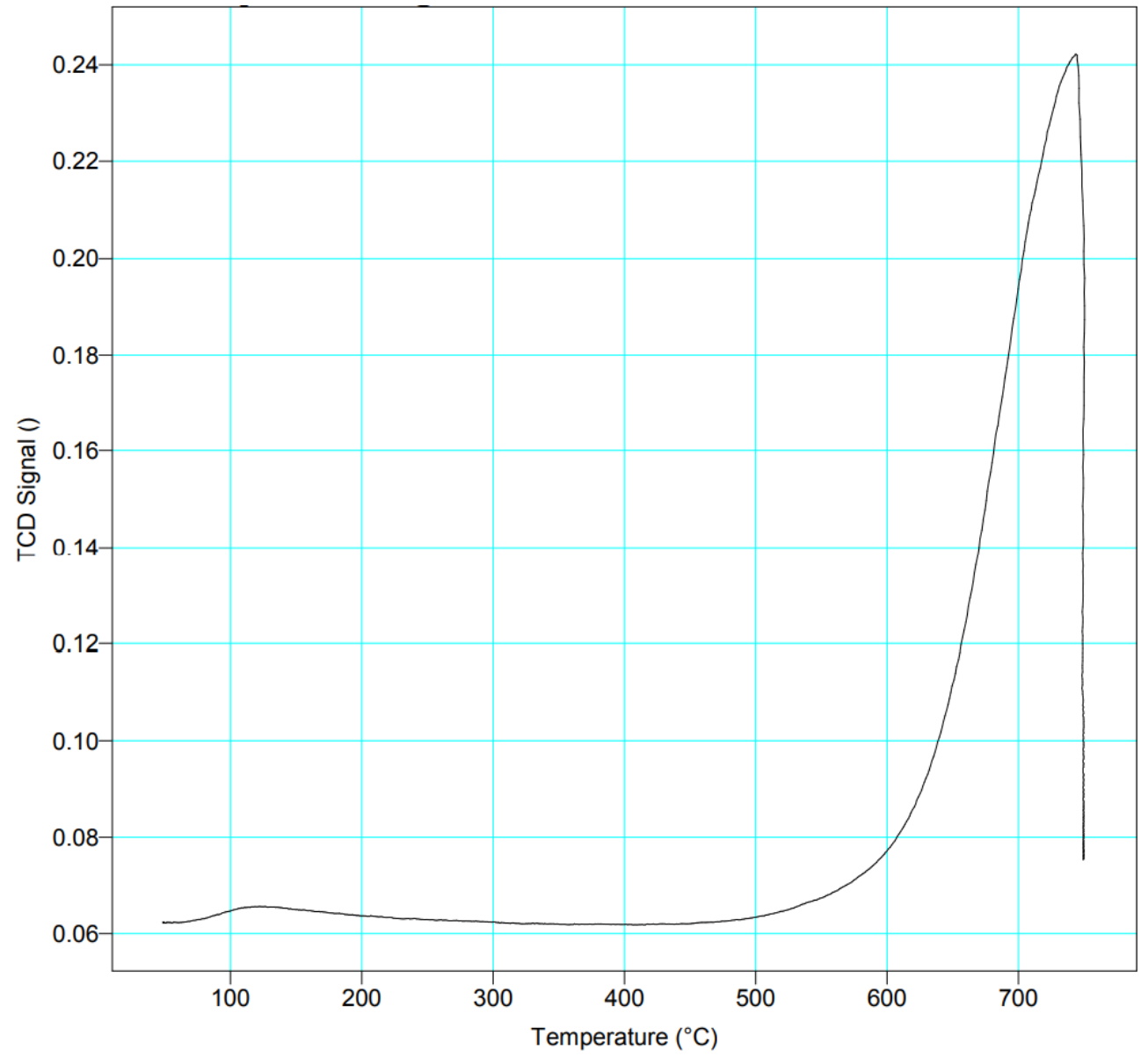

Figure 4.16: $\mathrm{CO}_{2}$-TPD intensity vs. temperature plot for dried PRB coal char

All $\mathrm{Me}_{\mathrm{x}} \mathrm{O}_{\mathrm{y}} /$ char catalysts had strong, high-temperature TPD peaks, implying that there exists a high density of strong basic sites for $\mathrm{CO}_{2}$ chemisorption. Each sample showed a rather sharp, strong TPD peak beginning $\sim 550{ }^{\circ} \mathrm{C}$ was also observed peaking around $750{ }^{\circ} \mathrm{C}$. This strong $\mathrm{CO}_{2}$ chemisorption might have a beneficial effect on the catalytic activity for the DRM process. To enhance the $\mathrm{CO}_{2}$ adsorption characteristics of coal char, materials with high basicities and 
reforming capabilities were precipitated on the coal char's surface via incipient wetting. The amount of $\mathrm{CO}_{2}$ chemisorbed at room temperature was determined to be $97.34,95.65$, and 112.86 $\mu \mathrm{mol} \mathrm{CO} 2$ gram catalyst for, $\mathrm{CaMoO}_{4} / \mathrm{char}, \mathrm{SrMoO}_{4} /$ char, and $\mathrm{MoO}_{3} /$ char respectively. The $\mathrm{CO}_{2}$ TPD patterns for each $\mathrm{Me}_{\mathrm{x}} \mathrm{O}_{\mathrm{y}} /$ char catalyst were obtained as shown in Figures 4.17, 4.18, and 4.19, respectively.

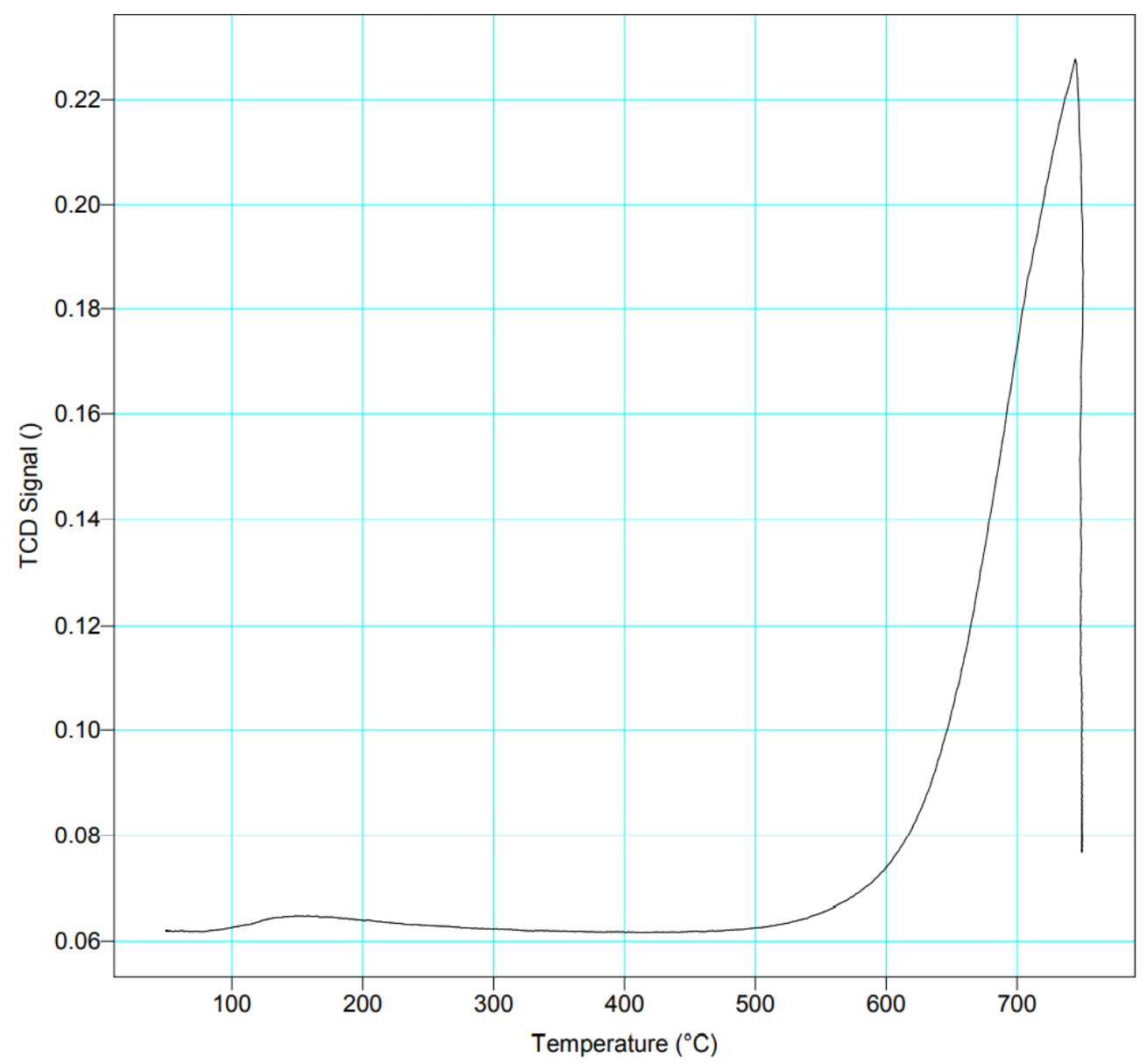

Figure 4.17: $\mathrm{CO}_{2}$-TPD intensity vs. temperature plot for dried PRB coal char impregnated with 5 wt $\%$ of $\mathrm{CaMoO}_{4}$. 


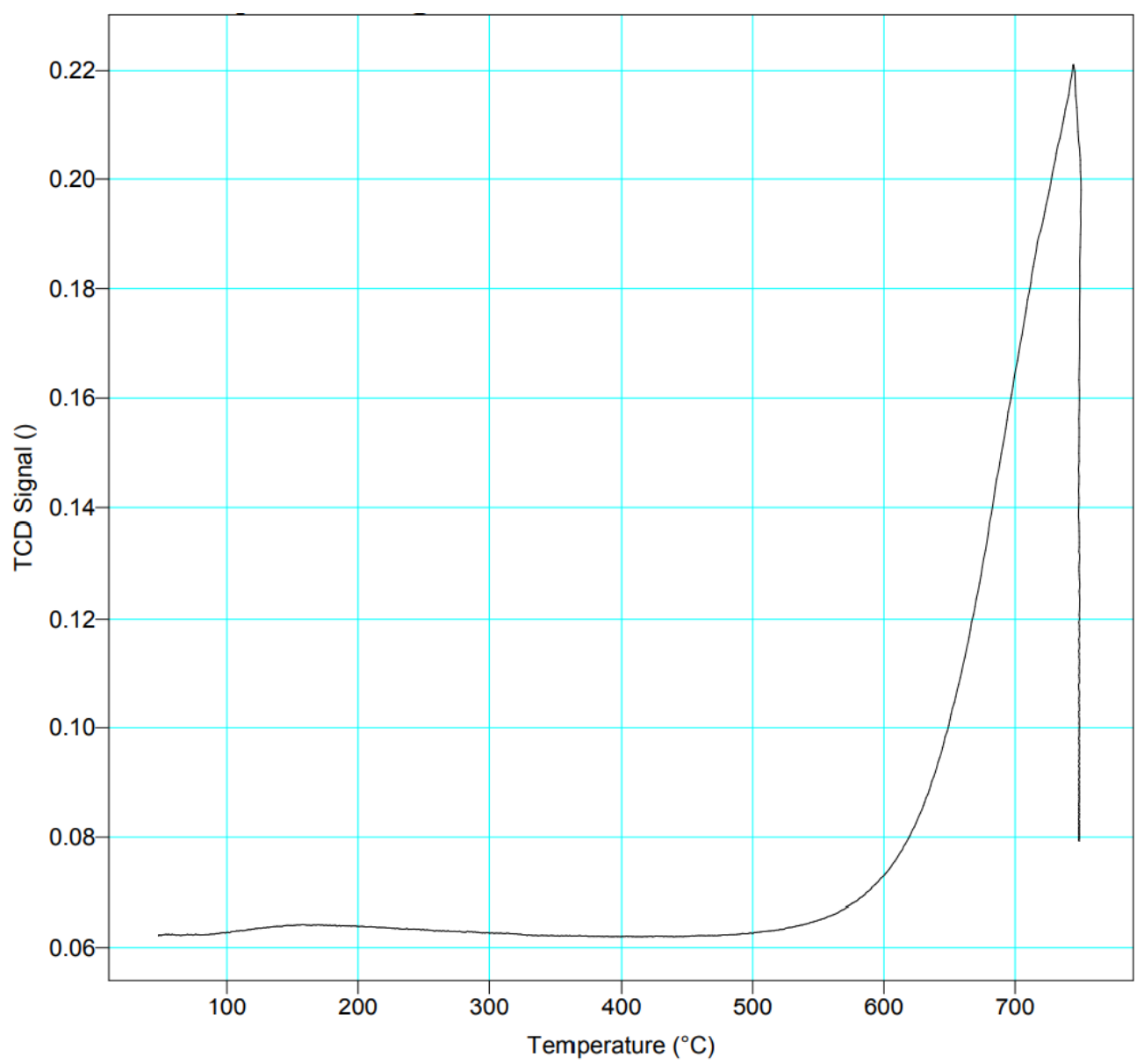

Figure 4.18: $\mathrm{CO}_{2}$-TPD intensity vs. temperature plot for dried PRB coal char impregnated with 5 wt $\%$ of $\mathrm{SrMoO}_{4}$. 


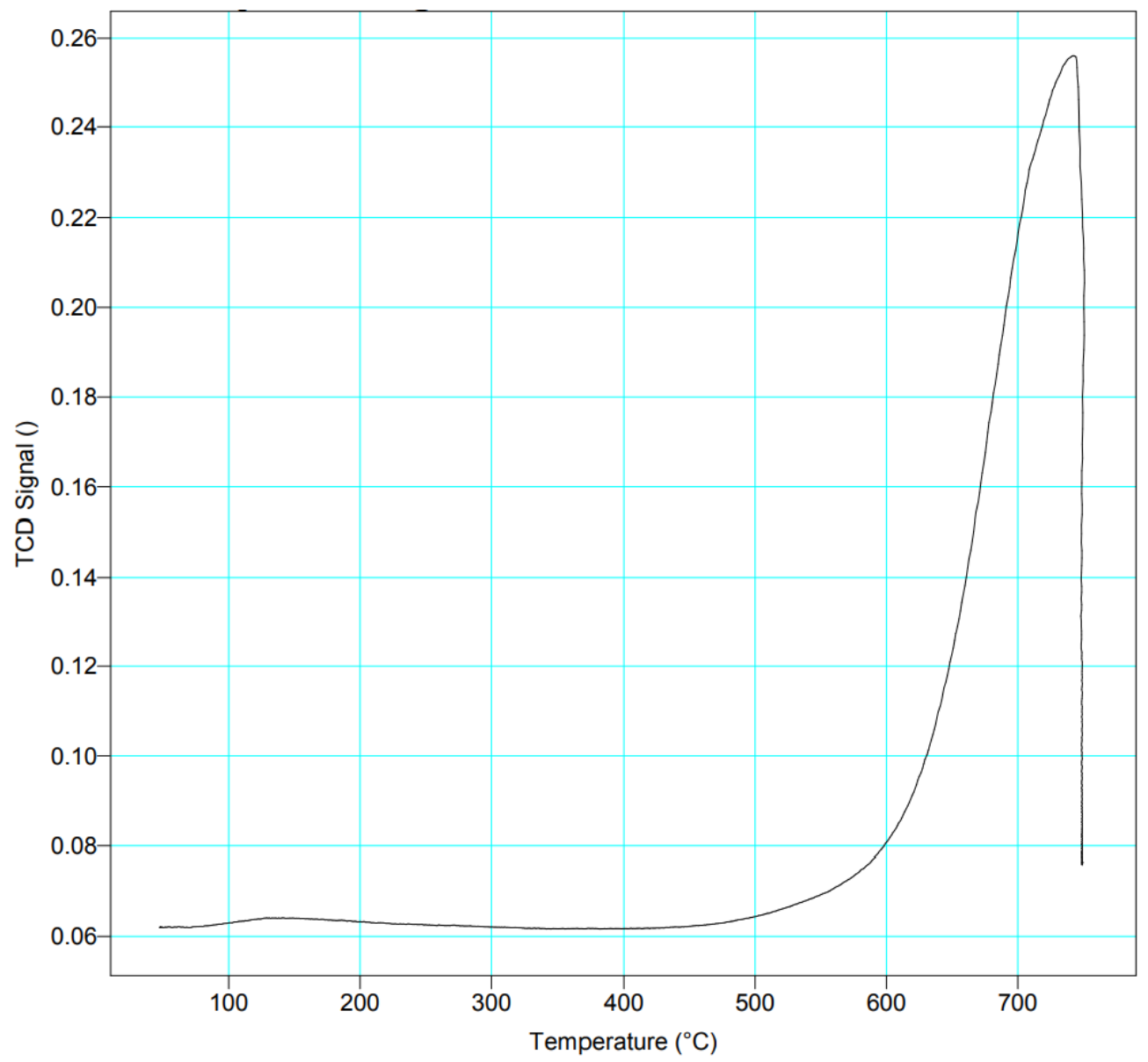

Figure 4.19: $\mathrm{CO}_{2}$-TPD intensity vs. temperature plot for dried PRB coal char impregnated with 5 $\mathrm{wt} \%$ of $\mathrm{MoO}_{3}$.

Expectedly, each material demonstrated similar $\mathrm{CO}_{2}$ chemisorbed due to most of the sample being composed of PRB coal char. However, $\mathrm{CaMoO}_{4} /$ char and $\mathrm{SrMoO}_{4} /$ char had a slightly lower TPD peak compared to coal char and $\mathrm{MoO}_{3} /$ char. Each Mo catalyst consisted of $5 \mathrm{wt} \%$ of the total catalyst weight. The addition of the metal oxides would have to adsorb more $\mathrm{CO}_{2}$ than the coal char itself per unit area that it occupies. The lower peaks on these samples indicate that they have a lower density/weak basic sites on their surface than the surface area of coal char they cover, although this reduction in $\mathrm{CO}_{2}$ chemisorption does not fully indicated a related reduction in DRM reforming capabilities. $\mathrm{MoO}_{3} /$ char had the highest $\mathrm{CO}_{2}$ chemisorption peaks, slightly edging out bare PRB coal char, suggesting that the $\mathrm{MoO}_{3}$ on its surface does in fact adsorb $\mathrm{CO}_{2}$ more readily 
than the other Mo catalysts and might possibly have a beneficial effect on its DRM catalytic activity.

Ultimately, through MW induced dry reforming, dried PRB coal char can heat to reaction-ready temperature to reform both $\mathrm{CH}_{4}$ and $\mathrm{CO}_{2}$ into syngas. The localized temperature of the reactor was equivalent to a conventionally heated reactor, yet methane was able to reform. Reforming and coupling at such a low temperature are possible because of the temperature of the particles themselves, specifically the temperature on the surface of the particle. The surface temperature can be much hotter than that of the localized temperature (the temperature recorded off the entire bed during the reaction) within the MW reactor because the heat released from the particles are determined by their loss tangents, or their ability to absorb electromagnetic energy and release it in the form of heat. The coal char and its impurities can absorb the electromagnetic radiation and efficiently release it at a high temperature directly from its surface, instead of heating the surface from the system itself (Abdelsayed, Victor, et al., 2019). The higher surface temperature promotes the surface level reforming reactions, whereas systems heating catalyst beds conventionally never reach this peak since they are not playing to the strengths of the catalyst materials themselves. Section 4.4.4 of this chapter will provide further empirical evidence to suggest that these surface temperatures are much higher than the of the bulk catalyst bed's localized temperatures, where $\mathrm{SrMoO}_{4} / \mathrm{Char}$ operating at a $700{ }^{\circ} \mathrm{C}$ localized temperature melted the walls of a quartz tube. Additionally, the following sections will dive deeper into the observable interactions of the impregnated or mixing metal oxides on the surface of the coal char in DRM environments. Currently, the reactionary pathways effected by the changes in particle morphologies are difficult to infer upon, as much of these interactions between coal char, reforming gasses, and metal oxides have seldom been referenced within literature. The interactions, both physically and chemically, 
occurring between the coal char and metal oxide particles are incredibly complex and heavily debated. Nevertheless, the following sections have netted some theoretic conclusions by comparing GC MW reactor results to pre and post reaction SEM images of morphological changes.

\subsubsection{MWDRM Processing with $\mathrm{Fe}_{3} \mathrm{O}_{4}$ Mechanically Mixed and Impregnated on PRB Coal Char}

Several $\mathrm{Fe}_{3} \mathrm{O}_{4}$ particles were created in Chapter 3 that possessed atypical morphologies to that of naturally synthesized magnetite crystals. Of the shaped particles synthesized, $\mathrm{Fe}_{3} \mathrm{O}_{4}$ nanorods, microflakes, and microplatelets were chosen to be tested in the MW reactor vessel with and without PRB coal char. Section 4.4.1 tested the $\mathrm{Fe}_{3} \mathrm{O}_{4}$ particles without char, where the nanorods and microflake shaped particles depicted low conversion rates of the inlet gases and no carbon coupling. Both $\mathrm{Fe}_{3} \mathrm{O}_{4}$ nanoparticle morphologies experienced degradation post MWDRM processing. Here, two similar morphologies synthesized in Chapter 3, S3.3.2 and S3.3.3 micron octahedral and platelets, respectfully, particles were mechanically mixed with coal char to observe reforming improvements, alterations in outlet gaseous species, and determine any tertiary reactions are occurring between the metal oxides, char, and gasses in comparison to PRB coal char alone. Two additional samples were tested alongside the shaped particle; stock $\mathrm{Fe}_{3} \mathrm{O}_{4}$ nanoparticles as well as a sample the char was $\mathrm{Fe}_{3} \mathrm{O}_{4}$ was coprecipitated on its surface were used as the control samples for MWDRM processing. A list of the samples tested are in Table 4.2 below.

Table 4.2: $5 \mathrm{wt} \% \mathrm{Fe}_{3} \mathrm{O}_{4} / \mathrm{char}$ samples tested in a 5.85 GHz MW reactor operating at $600{ }^{\circ} \mathrm{C}$ under DRM processing conditions 


\begin{tabular}{|c|c|c|}
\hline Sample ID & Application & Particle Shape \\
\hline S4.1.1 & Mechanical Mix & Micron Octahedrons \\
\hline S4.1.2 & Mechanical Mix & Micron Platelets \\
\hline S4.1.3 & Mechanical Mix & Nanospheres (Stock) \\
\hline S4.1.4 & Coprecipitation & N/A \\
\hline
\end{tabular}

All samples were tested at the same reactionary conditions in both a conventional and MW heated reactor system under 200 SCCMs of nitrogen, methane, and carbon dioxide at a ratio of 2:1:1, respectively. The reactors were set to a localized temperature of $600{ }^{\circ} \mathrm{C}$ for approximately 1 hour of DRM processing. The product gasses were collected and analyzed using gas chromatography. The $\mathrm{Fe}_{3} \mathrm{O}_{4}$ /char sample's $\mathrm{CH}_{4}$ and $\mathrm{CO}_{2}$ conversion rates compared to plain PRB coal char are shown below in Figures 4.20 and 4.21, respectively.

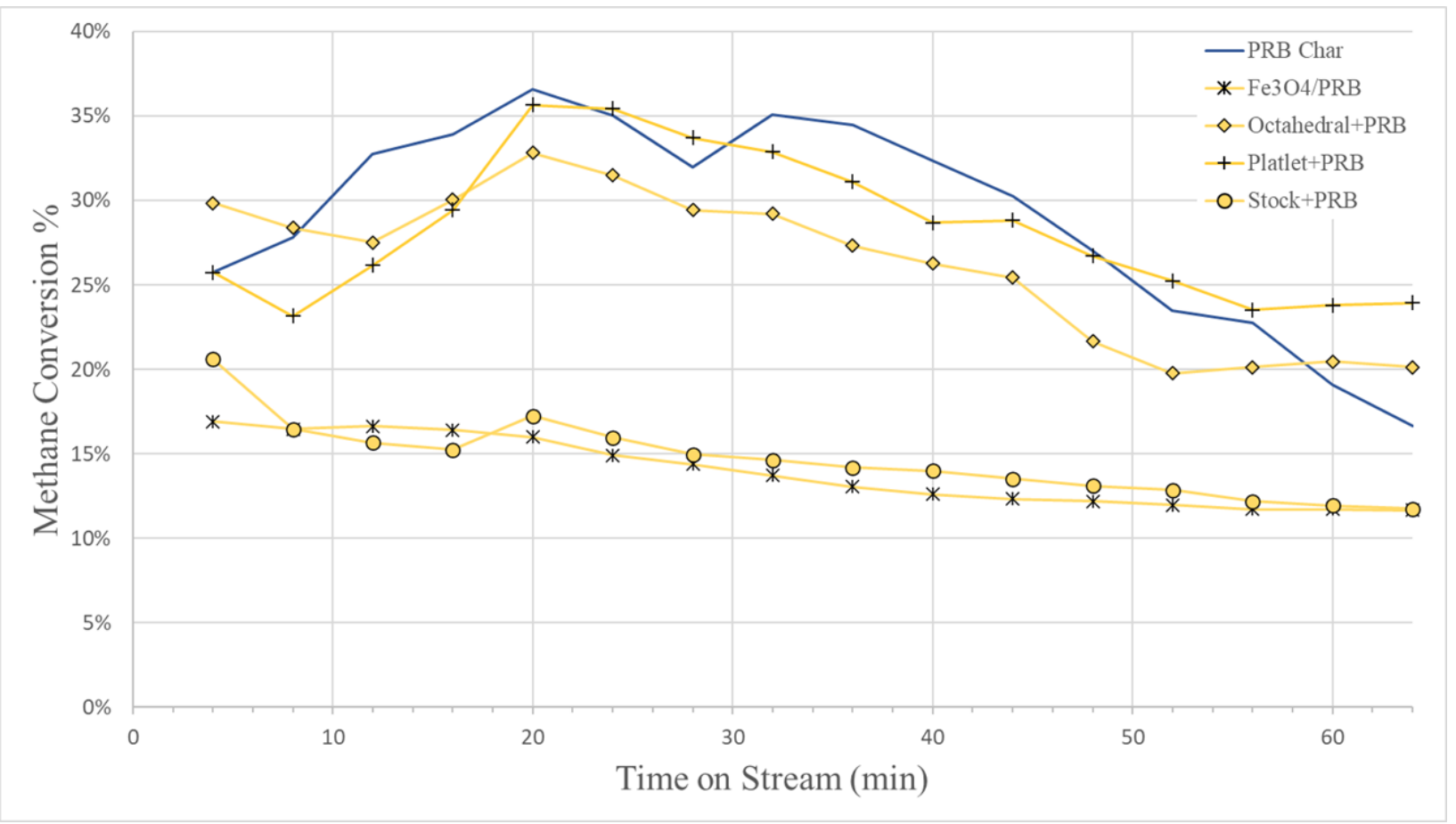

Figure 4.20: $\mathrm{CH}_{4}$ Conversion rates of $\mathrm{Fe}_{3} \mathrm{O}_{4} /$ char samples and char within a $5.85 \mathrm{GHz} \mathrm{MW}$ reactor in DRM conditions at $600{ }^{\circ} \mathrm{C}$. 


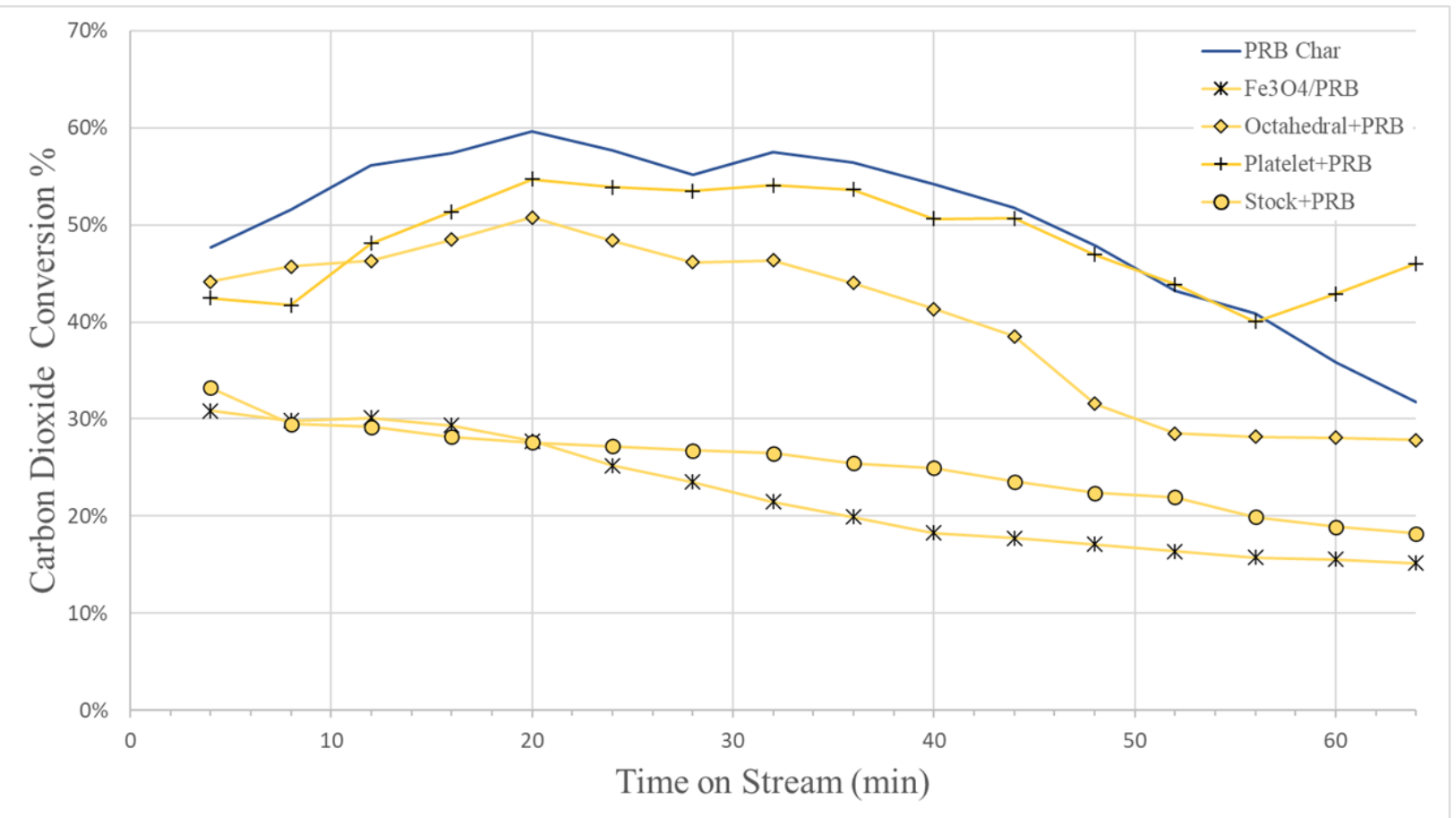

Figure 4.21: $\mathrm{CO}_{2}$ Conversion rates of $\mathrm{Fe}_{3} \mathrm{O}_{4} /$ char samples and char within a $5.85 \mathrm{GHz} \mathrm{MW}$ reactor in DRM conditions at $600^{\circ} \mathrm{C}$.

Similarly, to the bare, shaped $\mathrm{Fe}_{3} \mathrm{O}_{4}$ samples, S3.1.1 and S3.2.7, and coal char, each of the $\mathrm{Fe}_{3} \mathrm{O}-$ ${ }_{4} /$ char combinations were able to successfully reform the inlet gasses. However, none of the $\mathrm{Fe}_{3} \mathrm{O}_{4} /$ char samples were able to overcome the reforming/coupling capabilities of char alone. Each sample ultimately reduced the reforming capabilities of PRB coal char, except for samples S4.1.2 and $\mathrm{S} 4.1 .3$, where stock $\mathrm{Fe}_{3} \mathrm{O}_{4}$ was mixed with char mechanically at $5 \%$ of the total sample weight. Comparing catalytic differences between mechanically mixing and precipitating $\mathrm{Fe}_{3} \mathrm{O}_{4}$ with the PRB coal char in the MW reactor did not show conclusive difference favoring either method. Some of the mixed shaped samples (S4.1.2 and S4.1.3) preformed slightly better than other mixed samples such as $\mathrm{S} 4.1 .1$ which performed poorly alongside $\mathrm{Fe}_{3} \mathrm{O}_{4}$ impregnated char (S4.1.4). mechanically mixed samples contained the same wt $\%$ of $\mathrm{Fe}_{3} \mathrm{O}_{4}$, only having a difference in the morphology mixed with the coal char. Reforming to bare PRB coal char was mostly maintained 
with smaller particle sizes (S4.1.2, S4.1.3) opposed to larger ones (S4.1.1), where large, octahedral particles mixed with char performed the worst of the mixed samples.

The carbon coupling capabilities between the $\mathrm{Fe}_{3} \mathrm{O}_{4} /$ char particles was also lower than that of char alone. All samples were able to form ethylene and ethane, albeit at lower vol\% shown in Figures 4.22 and 4.23 , respectively.

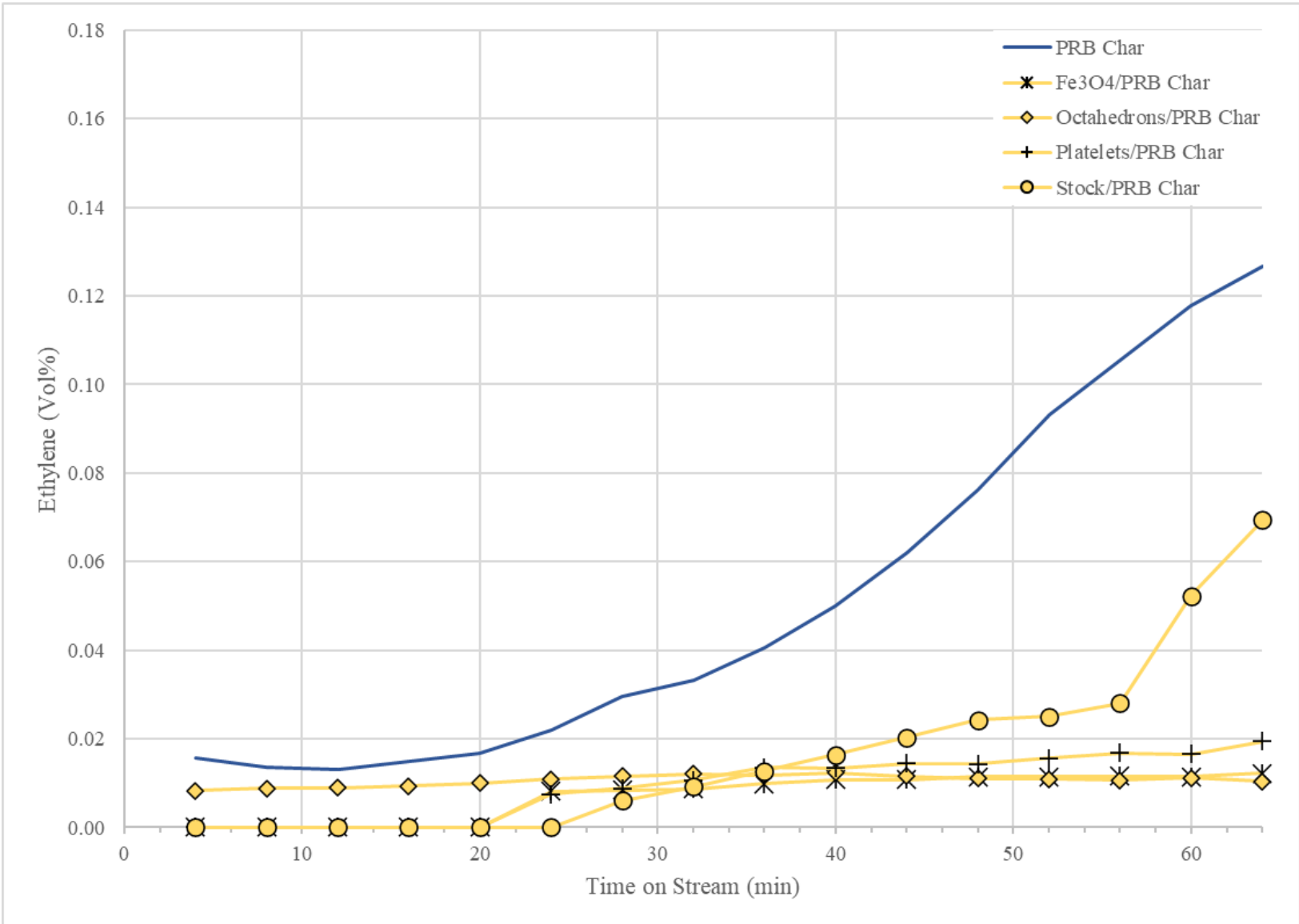

Figure 4.22: Ethylene outlet gas vol\% over $\mathrm{Fe}_{3} \mathrm{O}_{4} /$ char samples and char within a $5.85 \mathrm{GHz} \mathrm{MW}$ reactor in DRM conditions at $600^{\circ} \mathrm{C}$. 


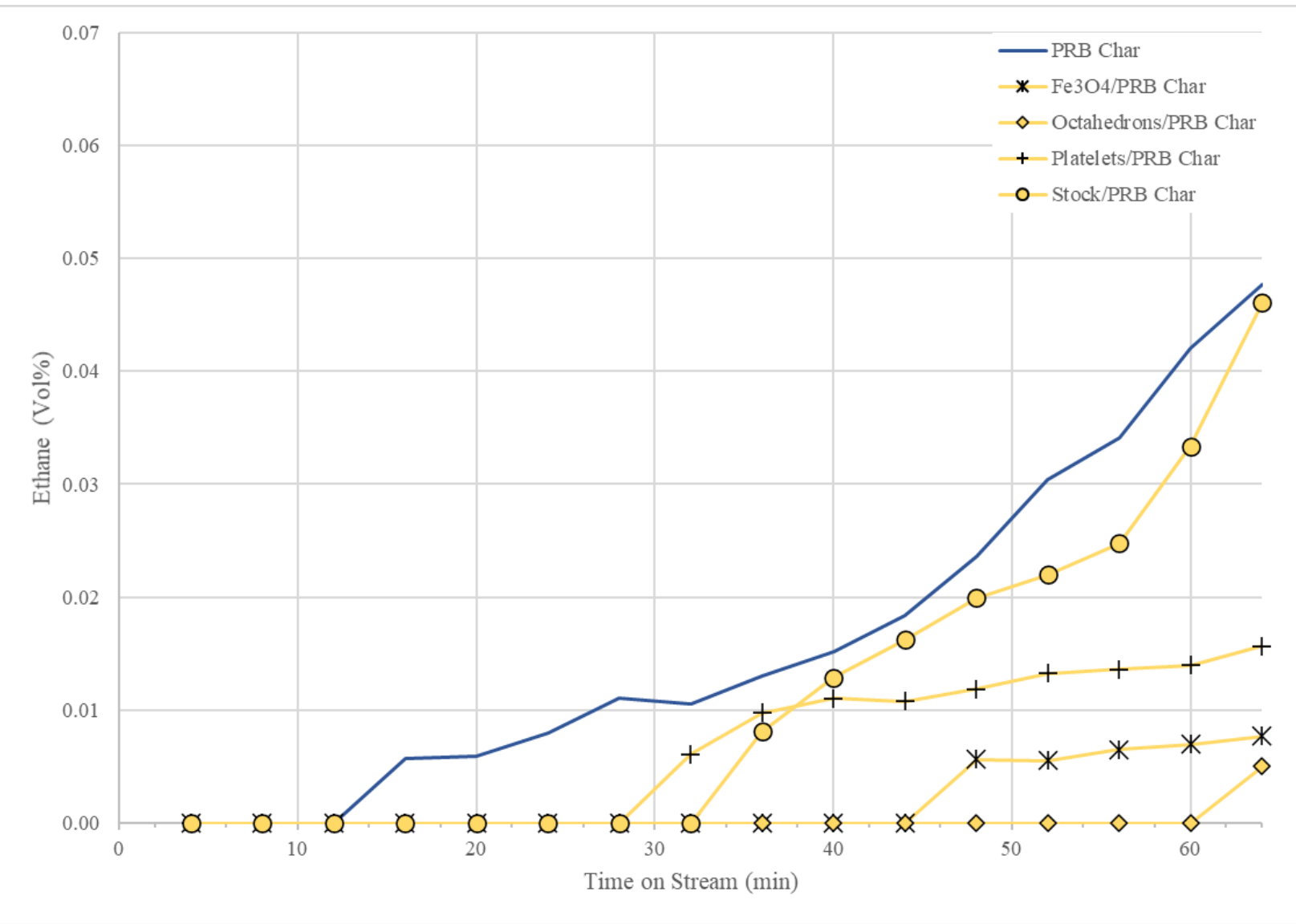

Figure 4.23: Ethane outlet gas vol\% over $\mathrm{Fe}_{3} \mathrm{O}_{4} /$ char samples and char within a $5.85 \mathrm{GHz} \mathrm{MW}$ reactor in DRM conditions at $600{ }^{\circ} \mathrm{C}$.

Looking to the pre and post reaction imaging of the $\mathrm{Fe}_{3} \mathrm{O}_{4} /$ char samples, all particles, both shaped and natural, experienced degradation in the DRM environment. Figure 4.24 below highlights SEM images of some major changes in morphology observed from each sample before and after their introduction in the MW reactor environment. 


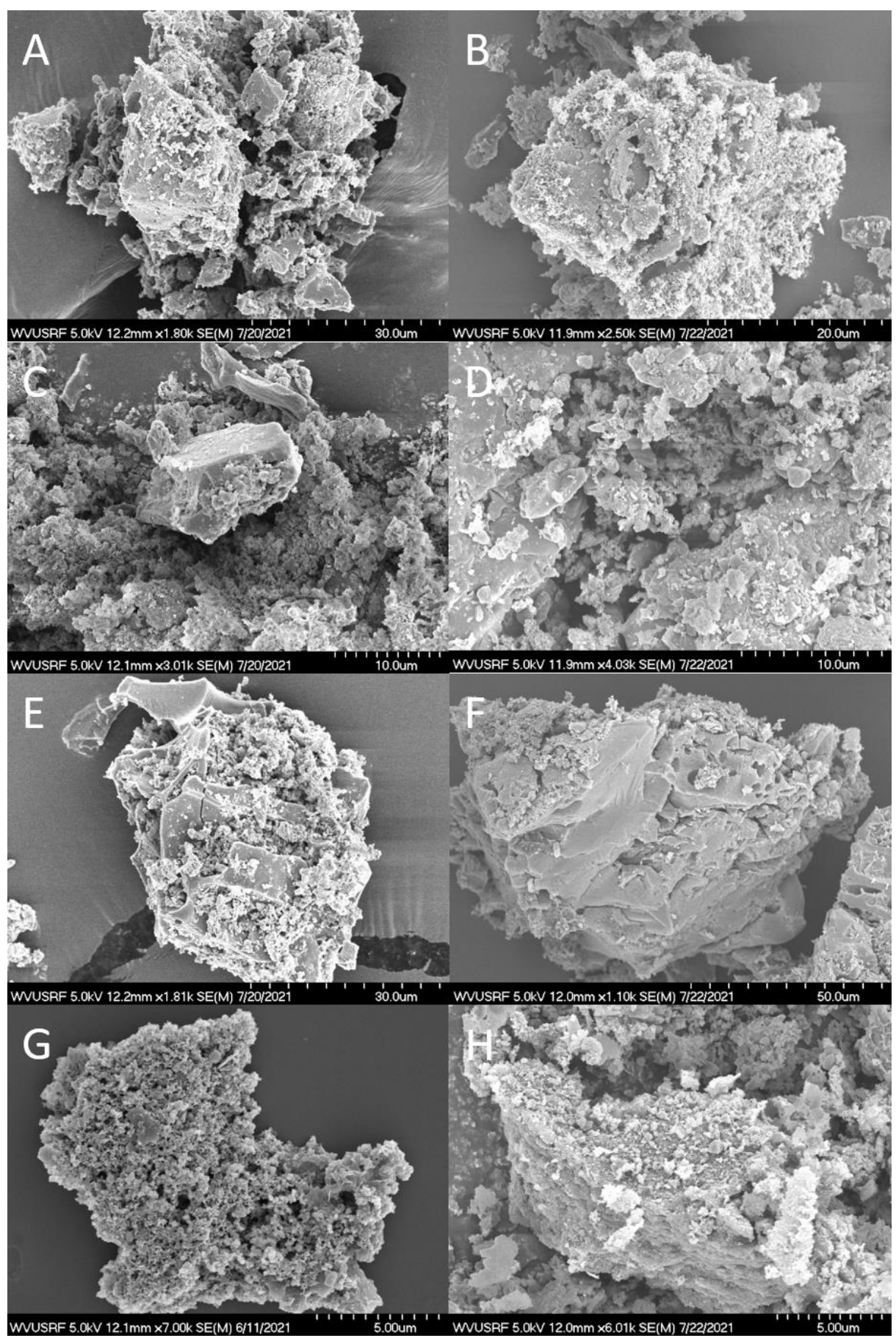

Figure 4.24: Pre (left) and post (right) reaction SEM imaging of $\mathrm{Fe}_{3} \mathrm{O}_{4} /$ char samples S4.1.1 (A,

B), S4.1.2 (C, D), S4.1.3 (E, F), and S4.1.4 (G, H). 
Firstly, samples 4.1.1 and 4.1.2 had both had shaped $\mathrm{Fe}_{3} \mathrm{O}_{4}$ micron particles mixed with the char for 30 minutes prior to entering the reactor. However, during the 30 minutes mechanical mixing process, it seems like much of each of morphology was damaged if not completely lost. Only small fragments of the octahedral particles were identifiable in S4.1.1 while none of the platelets from S4.1.2 are present in the char mixtures. The structure of each $\mathrm{Fe}_{3} \mathrm{O}_{4}$ particles post reaction when tested alone also exhibited low stability, but these examples degraded before entering the reactor, then afterwards they were eliminated.

Each example showed the same reduction of the smaller, flake-like char particle after MWDRM processing, leaving much larger, solid char particles. Notably, when examining the char particles themselves, two separate phenomena seem to be occurring. Many of the smaller, porous particles completely degrade leaving a fluffier mass of material behind as well as some smaller particulates of the char. The $\mathrm{Fe}_{3} \mathrm{O}_{4}$ is still present on some of the surfaces, but most becomes lost in much of the now smaller char particulates. The degradation of these particles could be leading to much of the reactionary losses as the carbon leaving the reaction as $\mathrm{CO}$ can no longer reform the $\mathrm{CH}_{4}$ molecules that would instead be doing. Secondly, the larger, solid char particles seem to agglomerate into much larger, some stretching to sizes $>200 \mu \mathrm{m}$ across. Much of the char's particles surface areas are highly reduced as these large agglomerates form, possibly leading to much of the reactionary losses observed. Additionally, the larger char particles are presumably much more stable than their smaller, flake-like counterparts, contributing less to methane reforming and $\mathrm{CO}$ generation. The appearance of large solid char particles is like what is seen during a pyrolysis reaction, where unreacted carbon repolymerizes into the solid char particles themselves. This is true for all post reactionary cases, both using $\mathrm{Fe}_{3} \mathrm{O}_{4}$ and $\mathrm{SrMoO}_{4}$ and char 
samples alone. Figure 4.25 displays these much larger particles, with smooth surfaces that had a limited presence prior to DRM processing.

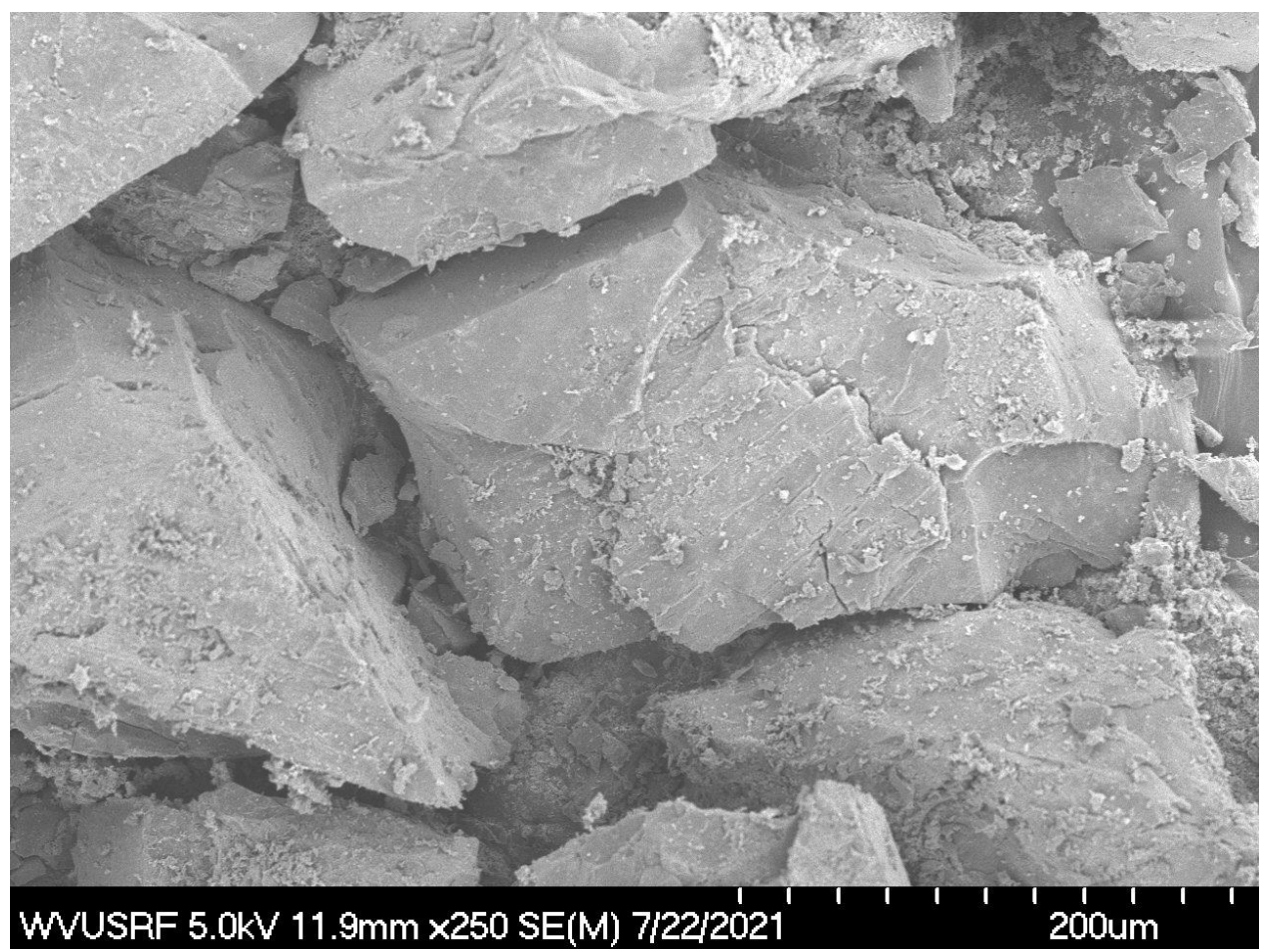

Figure 4.25: Example of PRB coal char agglomeration post MWDRM processing from $\mathrm{Fe}_{3} \mathrm{O}_{4}$ sample S4.1.4.

\subsubsection{MWDRM Processing with $\mathrm{SrMoO}_{4}$ Mechanically Mixed and Impregnated on PRB Coal Char}

The $\mathrm{SrMoO}_{4}$ particles created in Chapter 3 were chosen to be tested in the MW reactor vessel with PRB coal char for their ethylene coupling characteristics. Section 4.4.1 tested these faceted microspheres mixed with $\mathrm{SiC}$ to bring them to the appropriate temperature within the $\mathrm{MW}$ reactor. $\mathrm{SrMoO}_{4}$ alone was not able to reach the necessary reaction temperature without the use of SiC. Although microstructure of $\mathrm{SrMoO}_{4}$ was lost during the reaction, the particles did not lose the ability to couple carbon into ethylene and ethane. A list of the samples tested are in 
Table 4.3 below.

Table 4.3: $\mathrm{SrMoO}_{4} / \mathrm{Char}$ samples tested in a 5.85 GHz MW reactor operating at $600{ }^{\circ} \mathrm{C}$ under DRM processing conditions

\begin{tabular}{|c|c|c|c|}
\hline Sample ID & Application & Particle Shape & Wt\% on PRB Char \\
\hline $\mathrm{S} 4.2 .1\left(600^{\circ} \mathrm{C}\right)$ & Coprecipitation & N/A & $5 \%$ \\
\hline $\mathrm{S} 4.2 .1\left(700^{\circ} \mathrm{C}\right)$ & Coprecipitation & N/A & $5 \%$ \\
\hline $\mathrm{S} 4.2 .2$ & Coprecipitation & N/A & $10 \%$ \\
\hline $\mathrm{S} 4.2 .3$ & Mechanical Mix & Micron Spheres & $5 \%$ \\
\hline $\mathrm{S} 4.2 .4$ & Mechanical Mix & Micron Spheres & $10 \%$ \\
\hline
\end{tabular}

All samples were tested at the same reactionary conditions in both a conventional and MW heated

reactor system under 200 SCCMs of nitrogen, methane, and carbon dioxide at a ratio of 2:1:1, respectively. The reactors were set to a localized temperature of $600{ }^{\circ} \mathrm{C}$ for approximately 1 hour of DRM processing. The product gasses were collected and analyzed using gas chromatography. No conclusive evidence of coupling or reforming was seen within conventionally heated DRM reactions at $600{ }^{\circ} \mathrm{C}$, as these tests were conducted only for control comparison purposes. Data sets on conventional reactor tests may be found in the supplemental material. Each sample was replicated with varied $\mathrm{SrMoO}_{4}$ impregnation weights to isolate any reactionary changes that could have occurred with the addition/removal of active sites between the coal char support and the $\mathrm{SrMoO}_{4}$ particles. S4.2.1 was tested at two temperatures, $600{ }^{\circ} \mathrm{C}$ and $700{ }^{\circ} \mathrm{C}$ to observe distinct differences in reforming/coupling capabilities within the MW reactor. The $\mathrm{SrMoO}_{4} /$ char sample's 
$\mathrm{CH}_{4}$ and $\mathrm{CO}_{2}$ conversion rates compared to PRB coal char and $\mathrm{SrMoO}_{4} / \mathrm{SiC}$ are shown below in Figures 4.26 and 4.27 , respectively.

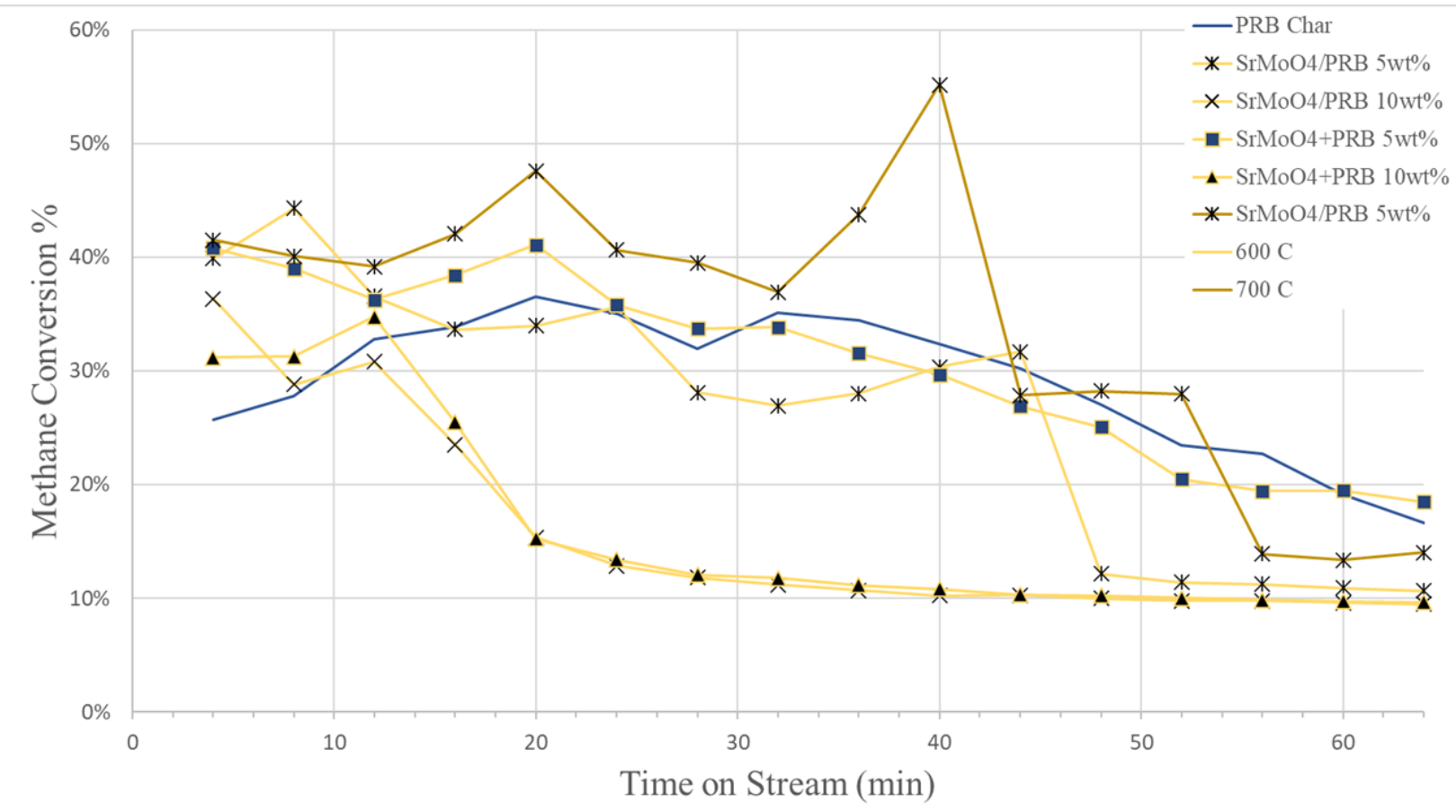

Figure 4.26: $\mathrm{CH}_{4}$ Conversion rates of $\mathrm{SrMoO}_{4} / \mathrm{Char}$ samples compared against bare PRB coal char and $\mathrm{SrMoO}_{4} / \mathrm{SiC}$ within a $5.85 \mathrm{GHz} \mathrm{MW}$ reactor.

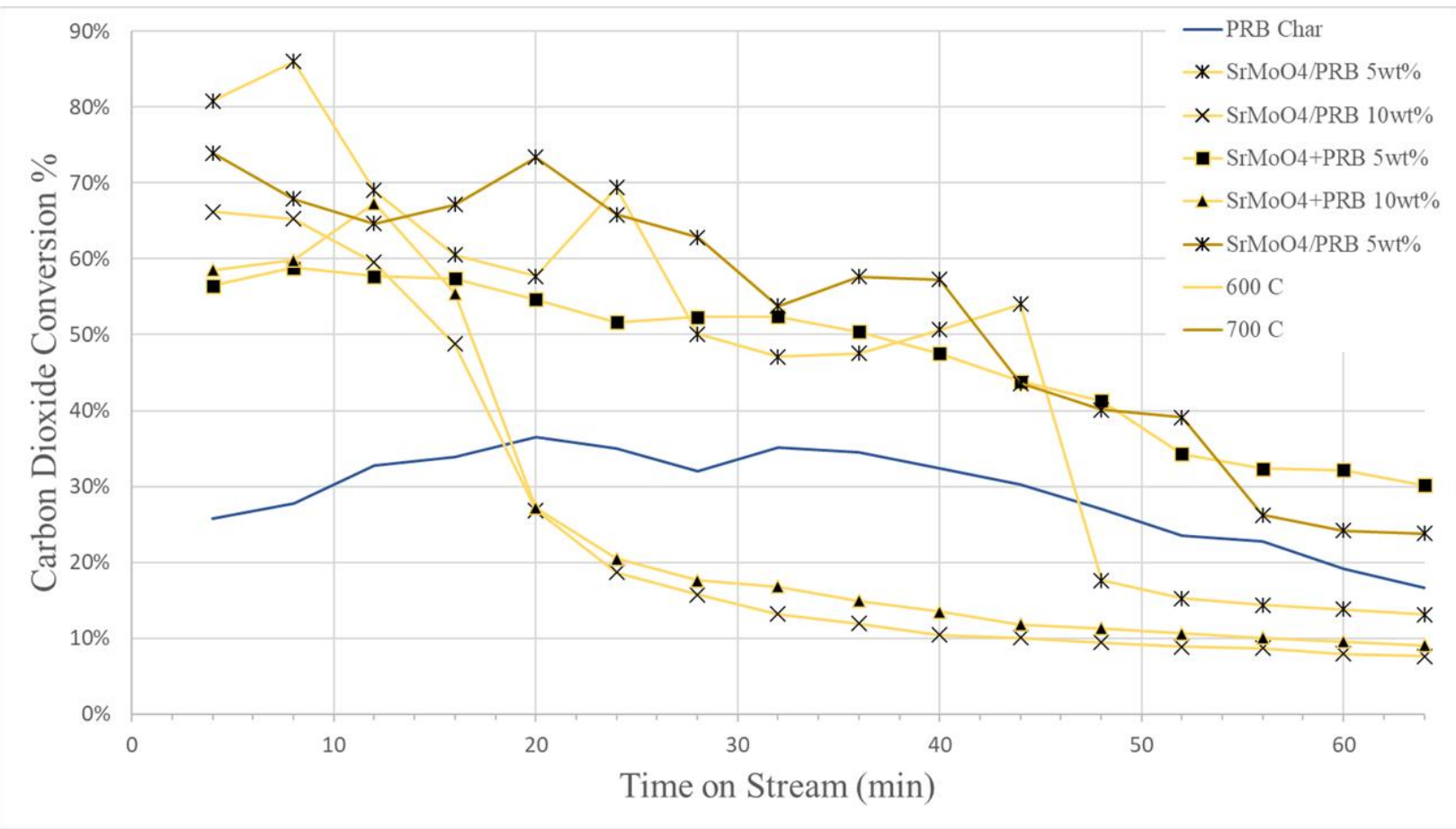


Figure 4.27: $\mathrm{CO}_{2}$ Conversion rates of $\mathrm{SrMoO}_{4} / \mathrm{Char}$ samples compared against coal char and $\mathrm{SrMoO}_{4} / \mathrm{SiC}$ within a $5.85 \mathrm{GHz} \mathrm{MW}$ reactor.

Each reaction was capable of reforming $\mathrm{CH}_{4}$ and $\mathrm{CO}_{2}$ into syngas, as well as showing some limited carbon coupling into ethylene and ethane. Samples S4.2.2 and S4.2.4 both containing 10 wt\% $\mathrm{SrMoO}_{4}$ experienced the lowest reforming of all the samples over the entirety of the test. Both started with higher reforming than the coal char and $\mathrm{SrMoO}_{4} / \mathrm{SiC}$ samples with average $\mathrm{CH}_{4}$ and $\mathrm{CO}_{2}$ reforming of $\sim 35.1 \%$ and $\sim 62.6 \%$, respectfully before the reforming of both reagents converged, quickly diminishing $\sim 43.5 \%$ after the first 20 minutes. Thereafter, both maintained low levels of reforming for the remainder of the reaction. All sample from these tests showed carbon coupling capabilities as well, however, only S4.2.1 operated at $700{ }^{\circ} \mathrm{C}$ was able to match the ethylene and ethane production of PRB coal char alone. Figure 4.28 and 4.29 displays the ethylene and ethane volumetric percentages, respectfully, of the product gasses from the MWDRM reaction compared with PRB coal char.

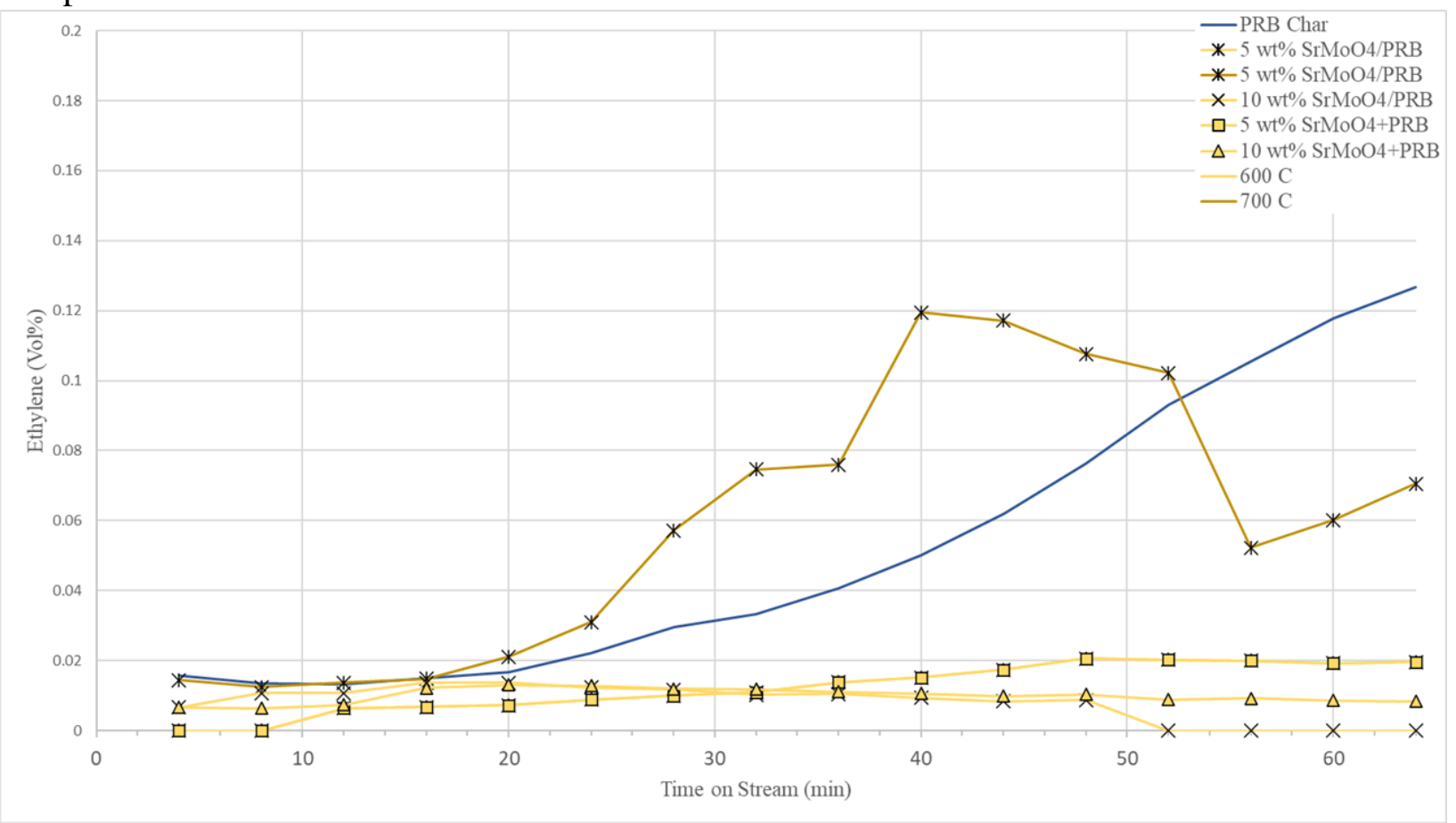


Figure 4.28: Ethylene outlet gas vol\% over $\mathrm{SrMoO}_{4} /$ char samples and char within a $5.85 \mathrm{GHz}$ MW reactor in DRM conditions.

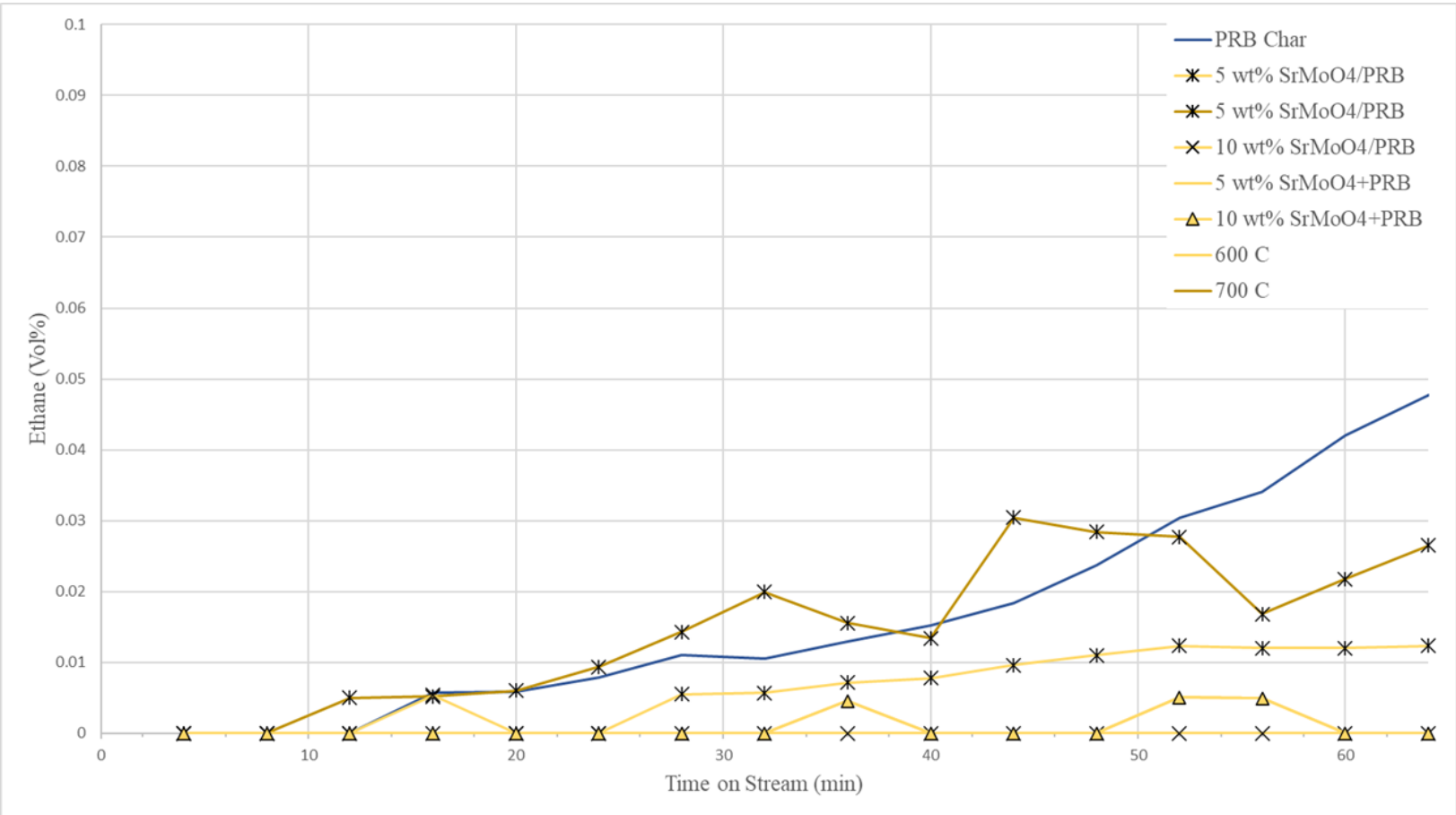

Figure 4.29: Ethane outlet gas vol\% over $\mathrm{SrMoO}_{4} /$ char samples and char within a $5.85 \mathrm{GHz}$ MW reactor in DRM conditions.

Like that of the $\mathrm{SrMoO}_{4} / \mathrm{SiC}$ sample, the microstructure of $\mathrm{SrMoO}_{4}$ on the surface of the char particles begins to coarsen and breakdown during the MW reaction. Figure 4.30 highlights SEM images of the $\mathrm{SrMoO}_{4}$ changes in morphology observed from each sample before and after their introduction in the MWDRM environment. 


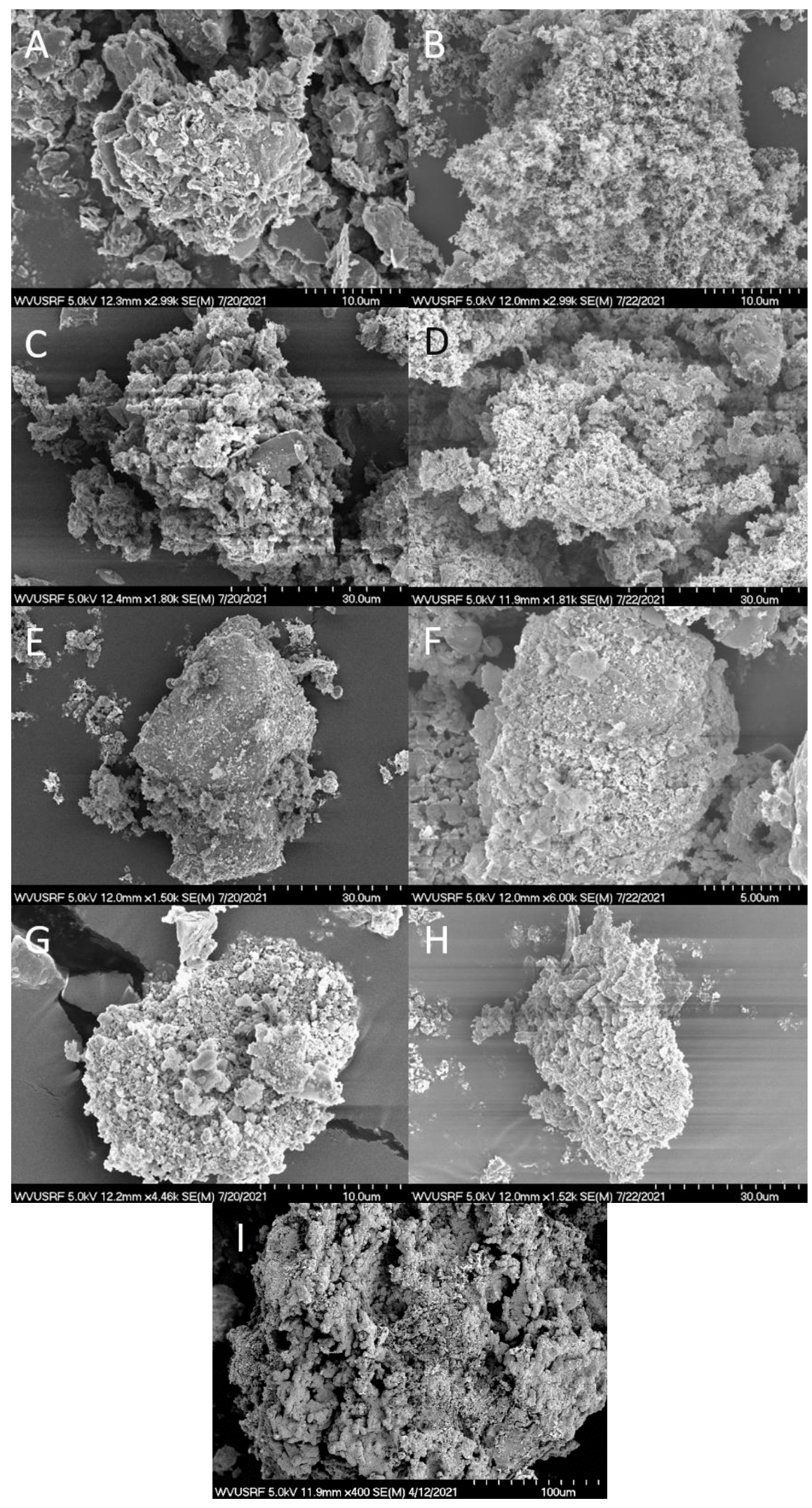


Figure 4.30: SEM imaging of pre (left) and post (right) reaction $\mathrm{SrMoO}_{4} /$ char samples S4.2.1

$$
\text { (A, B), S4.2.2 (C, D), 4.2.3 (E, F), S4.2.4 (G, H), and S4.2.1 at } 700{ }^{\circ} \mathrm{C}(\mathrm{I}) \text {. }
$$

Most of the $\mathrm{SrMoO}_{4}$ samples above lose their structure after the MWDRM reaction. The flakey $\mathrm{SrMoO}_{4}$ begins smoothing over into a larger mass that covers the outside of any nearby particles. This is especially true for the samples with a higher $\mathrm{SrMoO}_{4}$ to char ratio, as the outside of these samples were already highly decorated with $\mathrm{SrMoO}_{4}$. However, the samples using only 5 wt\% $\mathrm{SrMoO}_{4}$ depict a greater level of disfiguration comparatively, likely occurring from the greater mass of char induced with electromagnetic radiation, thus holding a higher temperature. Additionally, this greater mass of electromagnetically induced char has less $\mathrm{SrMoO}_{4}$ to heat in the $5 \mathrm{wt} \%$ samples, transferring more energy, and resulting in a more significant morphological change. This can especially be seen in S4.2.1 (Figure 4.30, I) SEM images, where the same $\mathrm{SrMoO}_{4}$ coarsening seen in the $\mathrm{SrMoO}_{4} / \mathrm{SiC}$ samples is present on the char surface.

The dried PRB coal char is handling a large portion of the reaction, shown to have the highest individual reforming/coupling capabilities in initial tests. As the $\mathrm{SrMoO}_{4}$ microstructure breaks down, it begins to cover the char particles, more importantly, the active sites responsible for reforming the reagent gasses. This essentially self-poisons the catalysts. The $10 \mathrm{wt} \%$ samples experienced faster poisoning due to the higher concentration of $\mathrm{SrMoO}_{4}$ covering the char's surfaces, restricting the active sites. The $5 \mathrm{wt} \%$ impregnated sample, S4.2.1, at both $600{ }^{\circ} \mathrm{C}$ and $700{ }^{\circ} \mathrm{C}$ also experienced the catalytic self-poisoning, but to a much lesser extent. All 5 wt $\%$ samples maintained higher $\mathrm{CH}_{4}$ reforming than that of char alone or $\mathrm{SrMoO}_{4} / \mathrm{SiC}$ for the first 40 44 minutes of the reaction before experiencing steep declines. Samples S4.2.1 at operating temperatures of $600{ }^{\circ} \mathrm{C}$ and $700{ }^{\circ} \mathrm{C}$, as well as sample $\mathrm{S} 4.2 .3$ maintained higher $\mathrm{CH}_{4}$ conversions for the majority of the MW reforming process, averaging conversions of $41.3 \%, 33.6 \%$, and 
$35.2 \%$, respectively, compared to char and $\mathrm{SrMoO}_{4} / \mathrm{SiC}^{\prime}$ s average conversions of $32.4 \%$ and $20 \%$, respectfully.

$\mathrm{SrMoO}_{4}$ is typically a very stable material comparatively to the difference of its parts, with a sublimation temperature of $1300-1530^{\circ} \mathrm{C}$ (Kazenas, E.K., Tsvetkov, Y.V., Samoilova, I.O. et al., 2008). Although the alterations to its morphology are not fully unexpected, especially so with the degradation seen with the $\mathrm{Fe}_{3} \mathrm{O}_{4}$ particles, it does come to question why they are degrading at low operating temperatures of $600-700{ }^{\circ} \mathrm{C}$. The degradation is not directly caused by the $\mathrm{SrMoO}_{4}$ itself because it is not capable of overcoming $\sim 300{ }^{\circ} \mathrm{C}$ as mentioned previously. However, after examining the post reaction sample (S4.2.1) operated at $700{ }^{\circ} \mathrm{C}$, the quartz tube holding the particles was damaged where the sample was sitting. Emptying the tube exposed heavy etching on the inner diameter of the quartz walls, and upon further inspection, small quartz spheres had broken off the walls into the sample itself. Quartz has an incredibly high melting point of $1650{ }^{\circ} \mathrm{C}$, normally much higher than any DRM reaction. Although the local temperature of the reaction was held at $\sim 700^{\circ} \mathrm{C}$, the particles themselves were at a dramatically higher temperature than this, high enough to produce the quartz spheres in the post reaction sample seen in Figure 4.31.

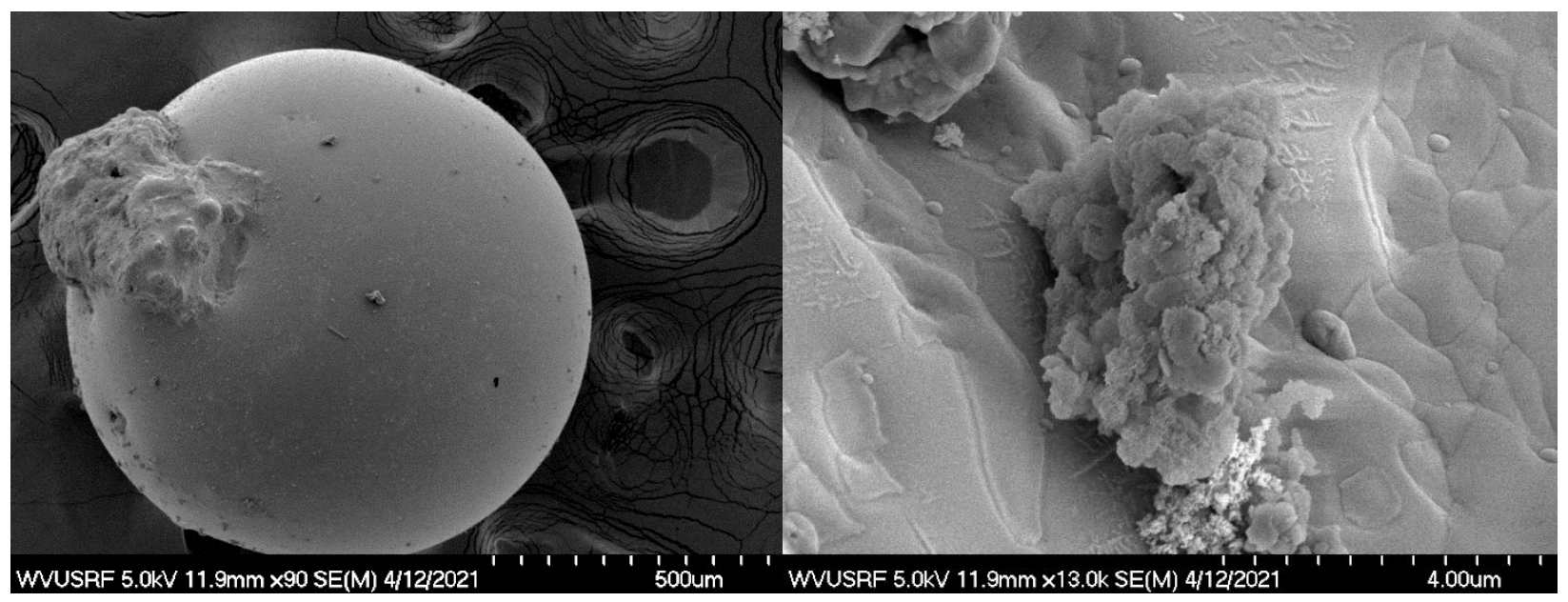

Figure 4.31: Quartz sphere discovered in sample S4.2.1 post MWDRM processing at $700{ }^{\circ} \mathrm{C}$

(left) and a close up of some $\mathrm{SrMoO}_{4}$ remaining on the glass sphere surface (right). 
Quartz melting at such a low local temperature can only be caused by the massive amount of energy the catalyst bed (namely the PRB coal char) is emitting. This example truly highlights one of the key advantages of microwave heating and how it can play a massive part in reactor design to minimize energy costs for a manufacturer. An ideal microwave catalyst could efficiently convert electromagnetic energy into temperatures unobtainable in conventional reactor designs using the same power supply. Higher catalyst bed temperatures increase reagent conversion all while maintaining a low energy requirement. Examples such as this emphasize the need for continued research into microwave reactor and catalyst design, as the potential operating cost savings from this technology is substantial.

\subsubsection{MWDRM Processing with $\mathrm{CaMoO}_{4}$ and $\mathrm{MoO}_{3}$ Impregnated on PRB Coal Char}

Two additional variants of molybdenum-based catalysts, $\mathrm{CaMoO}_{4}$ and $\mathrm{MoO}_{3}$, were tested in the MWDRM environment. All samples were impregnated on the surface of the PRB coal char at 5 wt $\%$ of the total sample mass. $\mathrm{CaMoO}_{4}$ was operated at both 600 and $700{ }^{\circ} \mathrm{C}$. A list of the samples tested are in Table 4.4 below.

Table 4.4: $\mathrm{CaMoO}_{4} / \mathrm{Char}$ and $\mathrm{MoO}_{3} / \mathrm{Char}$ samples tested in a $5.85 \mathrm{GHz} \mathrm{MW}$ reactor operating at $600{ }^{\circ} \mathrm{C}$ and $700{ }^{\circ} \mathrm{C}$ under DRM processing conditions

\begin{tabular}{|c|c|c|c|}
\hline Sample ID & Application & Temperature $\left({ }^{\circ} \mathbf{C}\right)$ & Wt\% on PRB Char \\
\hline S4.3.1 & Coprecipitation & 600 & $5 \%$ \\
\hline S4.3.2 & Coprecipitation & 700 & $5 \%$ \\
\hline S4.3.3 & Coprecipitation & 600 & $5 \%$ \\
\hline
\end{tabular}


All samples were tested at the same reactionary conditions in both a conventional and MW heated

SEM imaging of these particles are like that of the $\mathrm{SrMoO}_{4} /$ char samples, having a flaked, lichen-

like presence on the char's surface, seen in Figure 4.32.

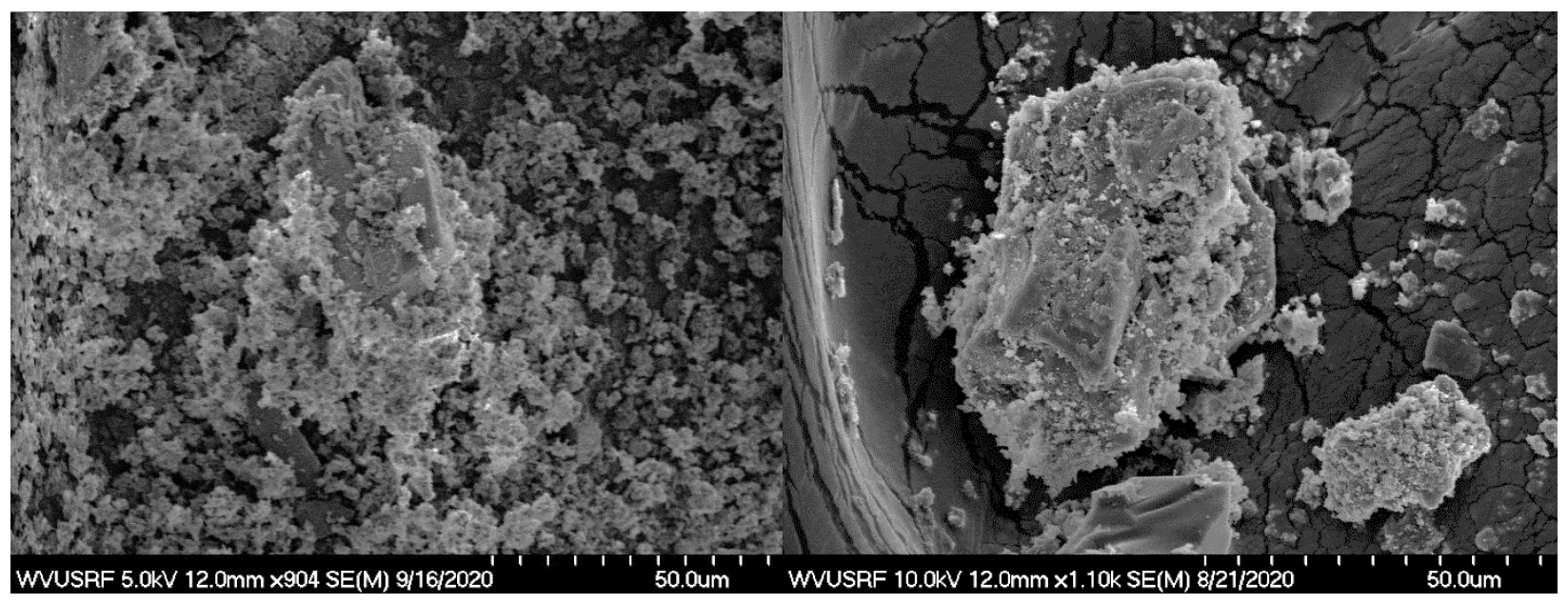

Figure 4.32: SEM imaging of $\mathrm{CaMoO}_{4} \mathrm{~S} 4.3 .1$ (left) and $\mathrm{MoO}_{3}$ (4.3.3 (right) impregnated on

PRB coal char.

Testing other Mo-based materials on char were executed to determine the DRM processing effects of changing or removing alkaline earth metals would have on the product of the reaction compared to the previously tested $\mathrm{SrMoO}_{4}$. Both samples underwent the same MWDRM processing as $\mathrm{SrMoO}_{4} / \mathrm{char}$ particles, remaining in the reactor for 64 minutes at a local temperature of $600{ }^{\circ} \mathrm{C}$. 
The $\mathrm{CH}_{4}$ and $\mathrm{CO}_{2}$ conversion rates of the samples versus char are depicted below in Figures 4.33 and 4.34 , respectively.

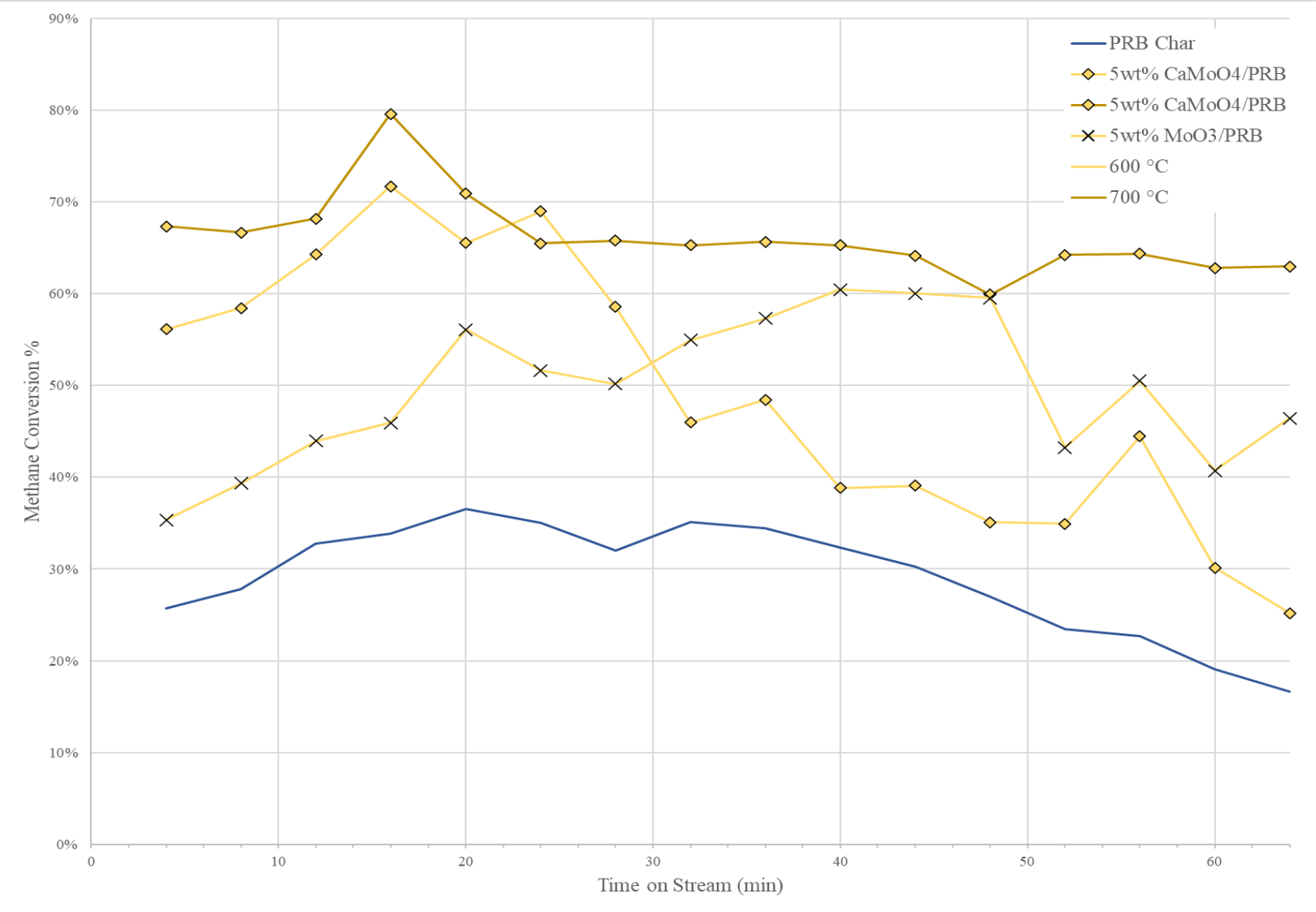

Figure 4.33: $\mathrm{CH}_{4}$ Conversion rates of $\mathrm{S} 4.3 .1$ and $\mathrm{S} 4.3 .2$ samples compared against bare PRB coal char within a $5.85 \mathrm{GHz} \mathrm{MW}$ reactor at 600 and $700{ }^{\circ} \mathrm{C}$. 


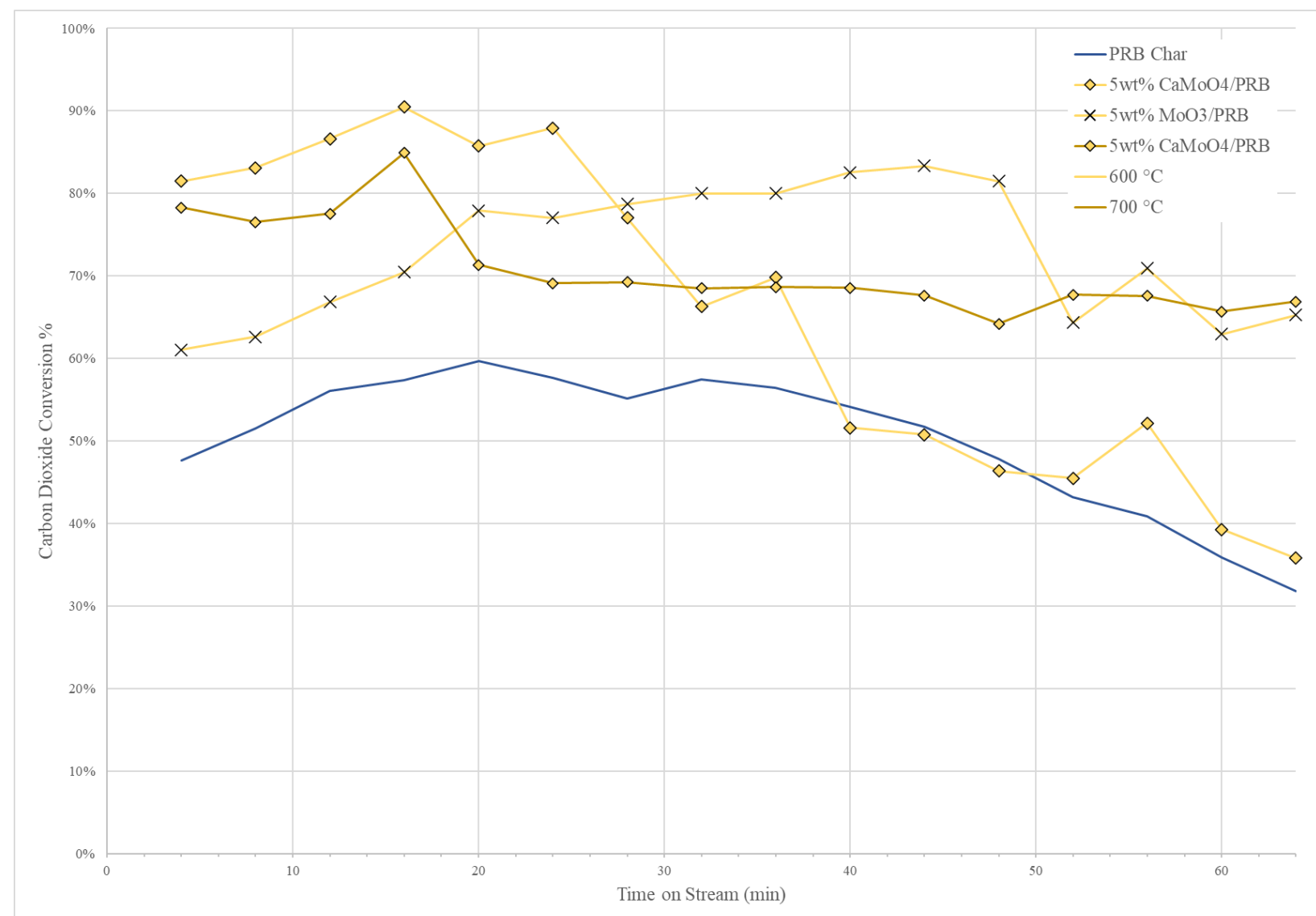

Figure 4.34: $\mathrm{CO}_{2}$ Conversion rates of S4.3.1 and S4.3.2 samples compared against bare PRB coal char within a $5.85 \mathrm{GHz} \mathrm{MW}$ reactor at 600 and $700{ }^{\circ} \mathrm{C}$.

The new $\mathrm{Mo} /$ char samples outperformed the previous $\mathrm{Fe}_{3} \mathrm{O}_{4} /$ char and $\mathrm{SrMoO}_{4} /$ char samples in reactionary longevity and overall $\mathrm{CH}_{4}$ and $\mathrm{CO}_{2}$ reforming capabilities. Both examples were able to continue reforming the reagent gasses at greater than or equivalent conversion rates compared to bare char. S4.3.1 reformed at a higher rate initially, then followed a similar conversion trend to the PRB coal char, while S4.3.3 reformed at a rate between $\mathrm{S} 4.3 .1$ and char yet remained relatively stable for the entirety of the processing time. The introductory reforming of S4.3.1 was the highest of all samples tested within this Chapter, peaking at $72.7 \%$ and $90.5 \% \mathrm{CH}_{4}$ and $\mathrm{CO}_{2}$ conversions, respectively, at after 16 minutes in the reaction environment, before steadily diminishing as the process continued. However, at $700{ }^{\circ} \mathrm{C}, \mathrm{CaMoO}_{4}$ sample $\mathrm{S} 4.3 .2$ was able to remain stable 
throughout the full reaction time with high rates of reforming. This was the only sample capable of stable reforming rates at $700{ }^{\circ} \mathrm{C}$. $\mathrm{SrMoO}_{4} /$ char and $\mathrm{MoO}_{3} /$ char samples at this temperature experienced unstable reforming and glowing in the packed bed. At $700{ }^{\circ} \mathrm{C}$, glowing occurs as the two materials are heating differently within the electromagnetic field, leading to hot spots within the bed. Although this same phenomenon was present for the $700{ }^{\circ} \mathrm{C}$ of $\mathrm{CaMoO}_{4} / \mathrm{char}$, it remained incredibly stable comparatively. $\mathrm{CaMoO}_{4} / \mathrm{char} \mathrm{S} 4.3 .2$ had the highest $\mathrm{CH}_{4}$ reforming average of all samples at $66.2 \%$. The $\mathrm{CO}_{2}$ average reforming was slightly lower at $71.4 \%$, however the reaction rate was much more consistent than all previously testing, disregarding S4.3.3 $\left(\mathrm{MoO}_{3} / \mathrm{char}\right)$. It was theorized that this high syngas reforming capability stemmed from the presence of $\mathrm{Ca}$ in the material. Calcium within catalytic materials, namely $\mathrm{CaO}$, have been noted for having high $\mathrm{CO}_{2}$ adsorption capabilities (Aika, K., \& Nishiyama, T., 1989; Dias, J., \& Assaf, J., 2003).

S4.3.3 was the only material able to keep a neutral trendline during the MWDRM processing time at $600{ }^{\circ} \mathrm{C}$, even concluding the test with a higher reforming rate than how it began. S4.3.3 only had $\mathrm{MoO}_{3}$ precipitated on its surface. $\mathrm{MoO}_{3}$ molecules have well documented DRM reactionary capabilities. Exposure to $\mathrm{CH}_{4}$ initiates a carburization reaction, converting the $\mathrm{MoO}_{3}$ into $\mathrm{MoO}_{\mathrm{x}} \mathrm{C}_{\mathrm{y}}$ and $\mathrm{Mo}_{2} \mathrm{C}$, both of which have recorded capabilities of upgrading $\mathrm{CH}_{4}$ into value-added fuels when placed on the appropriate substrate. This would explain the reforming rate increase through the first 48 minutes of the reaction, as the $\mathrm{MoO}_{3}$ on the char's surface continue to convert into $\mathrm{MoO}_{\mathrm{x}} \mathrm{C}_{\mathrm{y}}$ and $\mathrm{Mo}_{2} \mathrm{C}$ which are better at converting the reagent gasses. After 48 minutes elapse, the reaction rate begins to dip, which would be accredited to the loss of free moving carbon in the char matrix eroding from the $\mathrm{C}(\mathrm{s})+\mathrm{CO}_{2}$ reaction, like the char sample. Unlike the $\mathrm{SrMoO}_{4}$ samples, neither $\mathrm{CaMoO}_{4}$ nor $\mathrm{MoO}_{3}$ seem to be self-poisoning the catalyst bed, instead assisting the in the 
reforming reaction by working in unison with the char substrate. Since neither were able to heat in the electromagnetic environment, the char is providing the necessary scaffolding and bulk heating distribution for these materials to participate in the reaction, without covering char's active sites to reform the reagent gasses. However, there are noticeable losses in ethylene and ethane formations with these sample, both falling below the vol\% capable with char alone. The comparison between the outlet gas volumetric percentages of ethylene and ethane for samples S4.3.1, S4.3.3, and coal char are shown below in Figures 4.35 and 4.36, respectively.

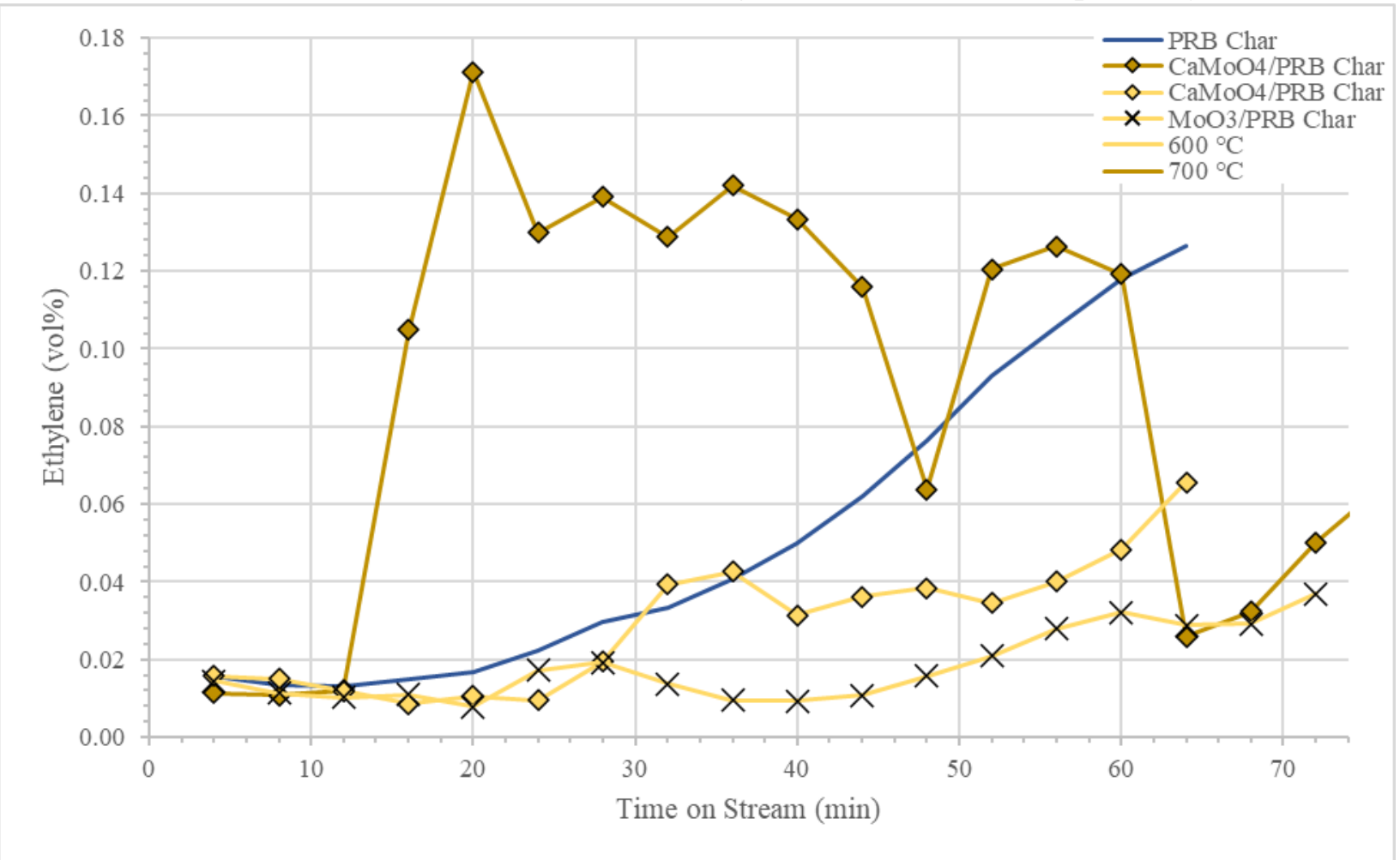

Figure 4.35: Ethylene outlet gas vol\% over S4.3.1, S4.3.2, S4.3.3, and coal char within a 5.85 GHz MW reactor in DRM conditions at 600 and $700{ }^{\circ} \mathrm{C}$. 


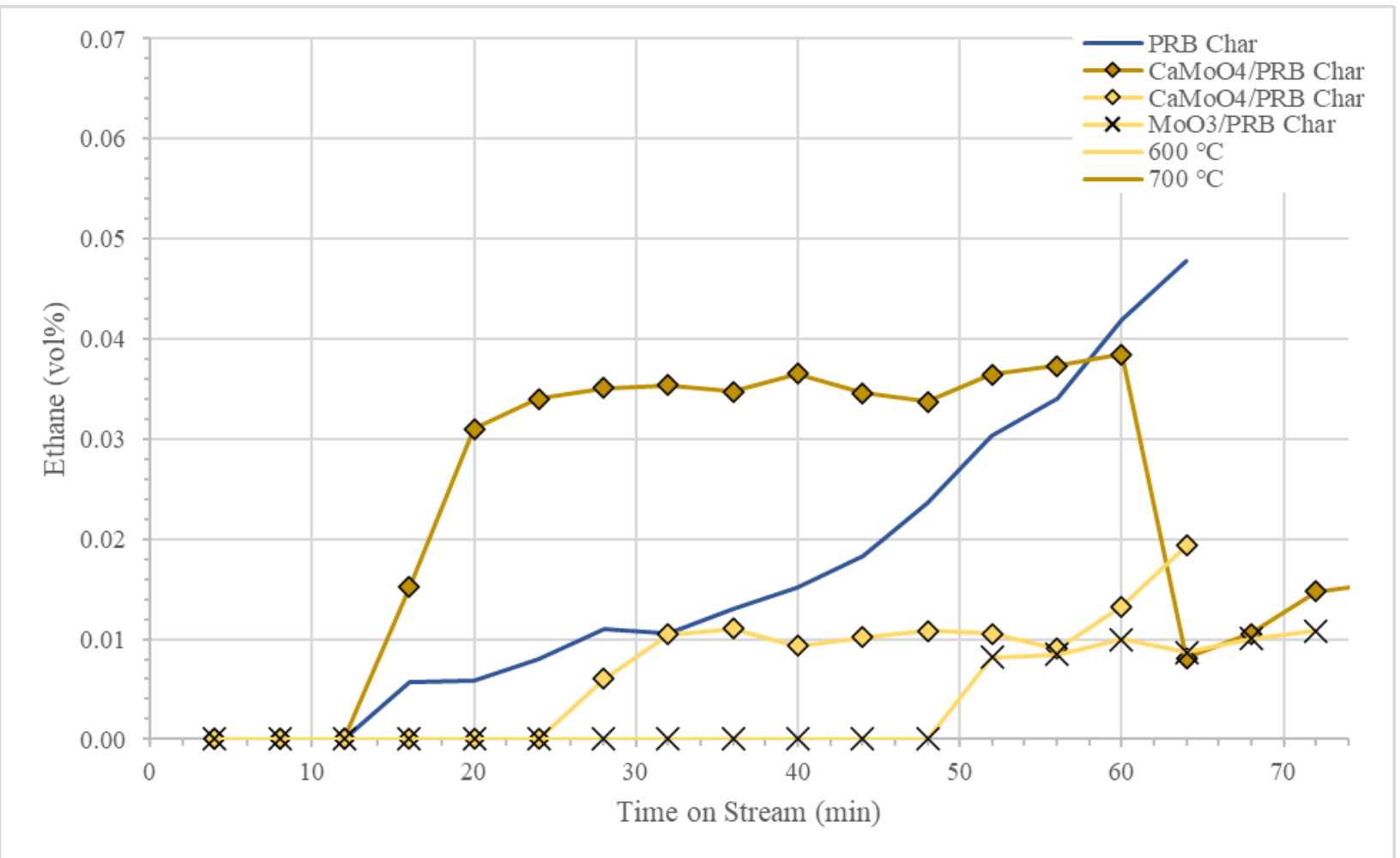

Figure 4.36: Ethane outlet gas vol\% over S4.3.1, S4.3.2, S4.3.3, and coal char within a $5.85 \mathrm{GHz}$ MW reactor in DRM conditions at 600 and $700{ }^{\circ} \mathrm{C}$.

Bare PRB coal char has consistently produced the most ethylene and ethane of all the metal oxide/char samples, including S4.3.1 and S4.3.3, however, S4.3.2 at $700{ }^{\circ} \mathrm{C}$ had spikes higher than PRB char. Overall, the $700^{\circ} \mathrm{C}$ sample of $\mathrm{CaMoO}_{4} / \mathrm{PRB}$ char did have more coupling than all other examples, the formation of ethylene and ethane were very unstable. Therefore, making a conclusive determination of the coupling effectiveness this sample was speculative at best. All samples, apart from S4.3.3, also experience two alterations as the MWDRM reaction occurs: a mass loss of the catalyst bed and a diminished reaction rate by the conclusion of the process. It is quite certain that the reforming reaction rate is diminishing during the MWDRM processing due to the removal of carbon within the PRB coal char. The carbon removal from the char within the catalyst sample enacts a domino effect on all proceeding interactions occurring within the reactionary environment. A loss of carbon leads to a reduction in basic active site density for 
reactant gasses to adsorb to alongside less solid and deposited carbon, from the char matrix and $\mathrm{CH}_{4}$ reforming, respectfully, for the $\mathrm{CO}_{2}$ to react. Additionally, as carbon is removed from the sample, the concentration of metal oxides within each sample rises. Although, this does not seem to pose as great of an effect on S4.3.1 and S.4.3.3, all preceding samples show this critically reducing reforming capabilities. With the removal of the less stable carbon from the system, large, low-surface area carbon particles remain after every reaction. The larger, dense particles remain since they seem to be fixed within a solid matrix compared to the smaller char pieces. These larger carbon particles do not have the same reforming capabilities as the small particles preceding them, however, reforming and carbon coupling is still capable. Most samples "bottom out" leveling at a lower reaction rate than where they began. It is certain that the electromagnetic induction of these larger char particles is still able to reform the reactant gases, albeit at a slower pace. However, it is less certain of why the coupling capabilities of most samples increase with time. This phenomenon cannot be linked with the formation of $\mathrm{Mo}_{2} \mathrm{C}$, since the carbon coupling rise is also seen with bare char samples than do not contain Mo. Instead, it is believed that the increase is instead associated with the removal of more mobile surface layer of carbon atoms that react with the reforming gasses. Afterwards, the surface environment is changed, exposing firmly fixed carbon atoms and impurities that cannot leave this new carbon matrix, but still have heating and reactionary capabilities within the MWDRM environment. These newly exposed, fixed carbon atoms interact with the $\mathrm{CH}_{4}$, now partially activating them into $\mathrm{CH}_{\mathrm{x}}$ radicals that then begin forming ethylene and ethane during a secondary reaction in the gas phase. Normally, under typical methane reforming conditions, there is not enough energy at $600{ }^{\circ} \mathrm{C}$ to initiate any dehydrogenation of the $\mathrm{CH}_{4}$ molecule. However, within the microwave reactor, the particles are at a temperature much higher than the recorded local temperature and are capable of methane dehydrogenation. 
Even at a local temperature of $600{ }^{\circ} \mathrm{C}$, some of these already partially activated $\mathrm{CH}_{3}{ }^{*}$ and $\mathrm{CH}_{2}{ }^{*}$ molecules that desorb from the char surface react with one another within the gaseous phase to form the trace amounts of ethylene and ethane in the final product. Naturally, it could be hypothesized that this output of ethylene and ethane would increase at a local temperature >600 ${ }^{\circ} \mathrm{C}$, since system temperatures $>700{ }^{\circ} \mathrm{C}$ are typically used in the production of value-added fuels and syngas alike. This assertion was shown to be true, as sample S4.2.1 in Section 4.4.4 tested at a local temperature of $700{ }^{\circ} \mathrm{C}$ depicted a spike in both ethylene and ethane coupling, both higher than that of PRB coal char alone. This carbon coupling would be assumed to be fully accredited to the char within the sample, as previous $\mathrm{SrMoO}_{4}$ samples at $600{ }^{\circ} \mathrm{C}$ did not show these capabilities and did not produce ethylene or ethane at a similar rate to bare PRB coal char. Although both coupling rates diminished after the spike, the reforming of S4.2.1 also fell at this time, due to the self-poisoning attributes of $\mathrm{SrMoO}_{4}$ and the erosion of available coal char described in these examples. However, when testing char alone at $700^{\circ} \mathrm{C}$, there was not a spike in ethylene or ethane, instead both vol\% fell well below what was seen with the $600{ }^{\circ} \mathrm{C}$ test. The higher reaction temperature coupled with the known erosion of carbon molecules in the char matrix from its reaction with the $\mathrm{CO}_{2}$ gas was concluded as the main reasons for this drop off in ethylene and ethane production. The overall conversion rate of $\mathrm{CH}_{4}$ and $\mathrm{CO}_{2}$ increased during the $700{ }^{\circ} \mathrm{C}$ test, both increasing slightly by $1.9 \%$ and $4.3 \%$, respectively. The increase in temperature supports higher adsorption rates of the reforming gasses on the char surface, allotting higher reaction rates thereafter. The surface of char at $700{ }^{\circ} \mathrm{C}$ is dispersing more heat than that of the $600{ }^{\circ} \mathrm{C}$ tests, activating $\mathrm{CH}_{4}$ more than previously achievable. The heightened local temperature of the system would promote the possibility of secondary gaseous phase reactions where carbon coupling tends to take place, but the increase in $\mathrm{CH}_{4}$ reforming into $\mathrm{H}_{2}$ decreases available methyl radicals. The 
decrease in methyl radicals from the high surface temperature of coal char and the rapid depletion from $\mathrm{CO}_{2}$ erosion of available coal char to catalyze the reforming gasses were concluded as the main causes for the coal char carbon coupling reduction of the $700{ }^{\circ} \mathrm{C}$ sample. Notably, S4.3.1 $\mathrm{SrMoO}_{4} / \mathrm{char}$ particles did not experience this same depletion in ethylene and ethane formation, instead spiking as previously stated. Since all else was kept constant and it is made clear that at $700{ }^{\circ} \mathrm{C}$ that char is not producing greater quantities of ethylene or ethane, $\mathrm{SrMoO}_{4}$ decorated had a heightened catalytic carbon coupling utility not seen in the $600{ }^{\circ} \mathrm{C}$ examples. $700{ }^{\circ} \mathrm{C}$ may be a more ideal temperature for $\mathrm{SrMoO}_{4}$ to couple methyl radicals into heavier, value added hydrocarbon fuels, but further tested would be required to make this assertion with the data available at this time.

\subsection{Conclusions}

\section{Summary of Research}

The purpose of this study was to compare the catalytic properties of several materials synthesized for dry reforming methane using microwave (MW) radiation and conventional heating methods. Materials synthesized with atypical morphologies in Chapter 3, $\mathrm{Fe}_{3} \mathrm{O}_{4}, \mathrm{CaMoO}_{4}$, and $\mathrm{SrMoO}_{4}$, were impregnated or mechanically mixed with dried PRB coal char and tested within the two reactor systems: one heated using MW radiation and the other using conventional heating. Plain molybdate $\left(\mathrm{MoO}_{3}\right)$ was also impregnated on coal char for comparisons sake to the other alkaline earth metal molybdates tested. These materials were introduced into $\mathrm{CH}_{4} / \mathrm{CO}_{2}$ dry reforming reactor environments and recorded to compare their catalytic influence on a dry reforming of methane through GC analysis. Several shaped catalytic particles were mixed with char for catalytic comparison and to determine if particle size and shape influenced a material's dry reforming capabilities. Bare PRB coal char was used as a control for the catalytic testing, serving as a baseline 
for of the other PRB coal char/metal oxide samples. The goals of this study were to observe reactionary changes between differently shaped catalysts, differentiate catalytic results between PRB coal char catalysts with metal oxides mixed and precipitated on its surface, and ultimately create a material that better utilizes PRB coal char and methane by converting them into a valueadded fuel source.

\subsubsection{Microwave Irradiated Shaped $\mathrm{Me}_{\mathrm{x}} \mathrm{O}_{\mathrm{y}}$ Catalysts for DRM Processing}

DRM processing was completed on four shaped particles: $\mathrm{CaMoO}_{4}$ micron platelets (S3.4.6), $\mathrm{SrMoO}_{4}$ faceted spheres (S3.5.1), and $\mathrm{Fe}_{3} \mathrm{O}_{4}$ nanoflakes and nanorods $\mathrm{S} 3.2 .7$ and S3.3.1, respectively in both a microwave and conventionally heated reactor. The four samples were synthesized to have thin, atypical morphologies produced in Chapter 3. Each was tested to serve as a baseline for how these materials would behave within DRM reactor conditions comparatively to the same compounds when impregnated on dried PRB coal char. Conventionally heated DRM processing was considered inconclusive for each material, as none of them were able to convert any of the reactant gasses at $600{ }^{\circ} \mathrm{C}$. This reactor temperature was far too low for any of the reactant gasses to a react with the catalyst beds. $\mathrm{Fe}_{3} \mathrm{O}_{4}$ particle beds were able to heat without the aid a substrate within the MW reactor, while $\mathrm{SrMoO}_{4}$ needed to be mixed with $\mathrm{SiC}$ to reach $600{ }^{\circ} \mathrm{C}$. Particles of different sizes and morphologies had small effects on reforming capabilities of $\mathrm{Fe}_{3} \mathrm{O}_{4}$, even though these physical changes altered the dielectric properties of the materials. At $600{ }^{\circ} \mathrm{C}$ in the MW irradiated reactor, $\mathrm{Fe}_{3} \mathrm{O}_{4}$ nanorods and nanoflakes ( $\mathrm{S} 3.1 .1$ and $\left.\mathrm{S} 3.2 .7\right)$ were able to reform $\mathrm{CH}_{4}$ and $\mathrm{CO}_{2}$ in some capacity, although $\mathrm{S} 3.1 .1$ showed higher, more stable rates of conversion. S3.2.7 nanoflake's conversions of $\mathrm{CH}_{4}$ and $\mathrm{CO}_{2}$ peaked initially at $5.1 \%$ and $5.7 \%$, respectively before rapidly falling 8 minutes into the test. $\mathrm{CO}_{2}$ conversion remained steady for much of the reaction, although only trace amount of $\mathrm{CO}$ gas was detected. $\mathrm{Fe}_{3} \mathrm{O}_{4}$ nanoflakes presented low 
average conversion rates of $\mathrm{CH}_{4}$ with no trace of $\mathrm{H}_{2}$ gas, instead showing the $100 \%$ selectivity for ethane with an average yield of $<0.02 \%$ in the outlet stream. $\mathrm{Fe}_{3} \mathrm{O}_{4}$ nanoflakes had limited reforming of $\mathrm{CO}_{2}$, only showing brief, negligible instances of $\mathrm{CO}$ in stream. $\mathrm{Fe}_{3} \mathrm{O}_{4}$ nanorods initial presented limited carbon coupling with a small yield of ethane for the first 8 minute of the reaction, yet also had low average $\mathrm{CH}_{4}$ and $\mathrm{CO}_{2}$ conversion into syngas. SEM imaging of $\mathrm{Fe}_{3} \mathrm{O}_{4}$ nanorods depicted a full morphological degradation of the nanorods, as they absent post-DRM reaction. SEM imaging of $\mathrm{Fe}_{3} \mathrm{O}_{4}$ nanoflakes somewhat maintained the plate/flake-like structures seen prior to MWDRM processing. The morphological changes were assumed to be directly associated with their reforming capabilities, as the nanorods had higher methane reforming rates that slowly diminished over time while the nanoflakes had small morphological changes as well as little reforming capabilities. TGA analysis did not suggest any significant suggestion of coke present on the material surfaces, both only losing $<0.01 \mathrm{mg}$ of weight after exposure to $10 \mathrm{SCCM}$ of air at $850{ }^{\circ} \mathrm{C}$ to burn off any carbon deposits. The higher reforming average was associated with the dielectric coefficient of the nanorod, 14.880, shaped particles which was significantly higher than the nanoflakes, 2.144. The greater loss tangent of the nanorod shaped particles allows for these $\mathrm{Fe}_{3} \mathrm{O}_{4}$ to absorb and disperse heat more efficiently than the nanoflakes. Since all else was kept constant during the reaction and both particles' shapes were of similar size, it was determined that the nanorod's altered morphology increased the dielectric properties to allow the MW heated surfaces of the particles to be at a higher temperature than the local temperature of the reactor.

The $\mathrm{SrMoO} / \mathrm{SiC}$ particles maintained a level $\mathrm{CH}_{4} / \mathrm{CO}_{2}$ reforming rates, averaging $18.1 \%$ and $29.1 \%$ conversion, respectively. $\mathrm{CH}_{4} / \mathrm{CO}_{2}$ conversion rates followed similar trends, gradually falling as the testing concluded. Catalytic degradation was not a result of coking during the reaction. TGA analysis showed no significant changes in weight after exposure to $10 \mathrm{SCCM}$ of air 
at $850{ }^{\circ} \mathrm{C}$ to burn off any carbon deposits. Additionally, the weight the catalyst bed remained unchanged from the start of the MWDRM processing to its conclusion. Catalytic losses were mainly attributed to phase alterations in the $\mathrm{SrMoO}_{4}$ 's microstructure that reduced exposed active sites. SEM imaging of the post-reaction $\mathrm{SrMoO}_{4} / \mathrm{SiC}$ particles confirmed particle coarsening and reductions in exposed surface areas compared to the facetted spheres. $\mathrm{SrMoO}_{4}$ was able to continue coupling methyl radicals into ethane and ethylene at a slow, steady rate despite significant deformation to the structure of the particle. $\mathrm{SrMoO}_{4}$ reactionary duality of reforming and carbon coupling was notably advantageous for the development of value-added fuels and required further testing with dried PRB char as a MW heating particle for the next series of testing.

\subsubsection{MWDRM Processing of $\mathrm{Me}_{\mathrm{x}} \mathrm{O}_{\mathrm{y}}$ Catalysts with Dried PRB Coal Char}

Shaped catalytic particles synthesized in Chapter 3 were impregnated or mechanically mixed with dried PRB coal char and tested within two reactor systems: one heated using MW radiation and the other using conventional heating. No materials tested within the DRM environment heated to $600{ }^{\circ} \mathrm{C}$ in a conventional reactor were able to react the reforming gasses. The reactor temperature was purposefully operated lower than a dry reforming reaction would normally occur to test potential catalytic effects with each of the materials at lower temperatures as well as provide a steep contrast to the MWDRM tests. All tests conducted in the convention reactor did not yield syngas or other volatile compounds. DRM reactions are typically conducted between $800-950{ }^{\circ} \mathrm{C}$, and $600{ }^{\circ} \mathrm{C}$ was too low for the reforming gasses to destabilize. Table 5.1 provides a comprehensive summary of each material tested and the average conversion rate of each reforming gas during a MWDRM reaction.

Table 5.1: Summary of All Materials Tested Within MWDRM Conditions and Average Conversion Rates of the Reforming Gasses 


\begin{tabular}{|cccccccc|}
\hline $\begin{array}{c}\text { Sample } \\
\text { ID }\end{array}$ & $\begin{array}{c}\text { Material } \\
\text { Tested }\end{array}$ & $\begin{array}{c}\text { Temp. of } \\
\mathbf{M W}{ }^{\circ} \mathbf{C}\end{array}$ & $\begin{array}{c}\text { Integration } \\
\text { Method }\end{array}$ & $\begin{array}{c}\text { Particle } \\
\text { Shape }\end{array}$ & $\begin{array}{c}\mathbf{M e}_{\mathbf{x}} \mathbf{O}_{\mathbf{y}} \\
\mathbf{W t \%}\end{array}$ & $\begin{array}{c}\text { Ave Conversion } \\
\mathbf{C H}_{\mathbf{4}} \%\end{array}$ & $\mathbf{C O}_{\mathbf{2}} \%$ \\
\hline Baseline & Char & 600 & N/A & N/A & 0 & 32.4 & 55.0 \\
\hline Baseline & Char & 700 & N/A & N/A & 0 & 35.3 & 59.3 \\
\hline S4.1.1 & $\mathrm{Fe}_{3} \mathrm{O}_{4}$ & 600 & Mech. Mix & Octahedrons & 5 & 14.9 & 24.9 \\
\hline S4.1.2 & $\mathrm{Fe}_{3} \mathrm{O}_{4}$ & 600 & Mech. Mix & Platelets & 5 & 28.9 & 45.5 \\
\hline S4.1.3 & $\mathrm{Fe}_{3} \mathrm{O}_{4}$ & 600 & Mech. Mix & Spheres (Stock) & 5 & 30.1 & 50.4 \\
\hline S4.1.4 & $\mathrm{Fe}_{3} \mathrm{O}_{4}$ & 600 & Precipitation & N/A & 5 & 15.7 & 27.5 \\
\hline S4.2.1 & $\mathrm{SrMoO}_{4}$ & 600 & Precipitation & N/A & 5 & 33.6 & 61.2 \\
\hline S4.2.1 & $\mathrm{SrMoO}_{4}$ & 700 & Precipitation & N/A & 5 & 41.3 & 62.5 \\
\hline S4.2.2 & $\mathrm{SrMoO}_{4}$ & 600 & Precipitation & N/A & 10 & 18.4 & 31.5 \\
\hline S4.2.3 & $\mathrm{SrMoO}_{4}$ & 600 & Mech. Mix & Spheres & 5 & 35.2 & 53.0 \\
\hline S4.2.4 & $\mathrm{SrMoO}_{4}$ & 600 & Mech. Mix & Spheres & 10 & 18.8 & 33.0 \\
\hline S4.3.1 & $\mathrm{CaMoO}_{4}$ & 600 & Precipitation & N/A & 5 & 56.0 & 75.5 \\
\hline S4.3.2 & $\mathrm{CaMoO}_{4}$ & 700 & Precipitation & N/A & 5 & 66.2 & 71.4 \\
\hline S4.3.3 & $\mathrm{MoO}_{3}$ & 600 & Precipitation & N/A & 5 & 50.5 & 74.6 \\
\hline
\end{tabular}

All tests with each material type within the microwave reactor were able to reach local temperatures of $600-700{ }^{\circ} \mathrm{C}$ and catalyze the reforming gasses into syngas with trace amounts of ethane and ethylene present in some experiments. Char was used as the baseline comparison for each material tested since each sample consisted of $>90 \%$ char by weight. It was concluded materials were at a much higher temperature than that of what was read on the temperature probe. This notion was further supported when unloading a $\mathrm{SrMoO}_{4} /$ char sample tested at $700{ }^{\circ} \mathrm{C}$, and the remaining particle bed had large spheres of quartz dispersed throughout the powder. The walls of the quartz sample tube had been damaged from where the sample was sitting, which would have required temperatures of $1450{ }^{\circ} \mathrm{C}$ or more to melt the quartz. Most materials mixed with or precipitated on char did not improve the catalytic abilities of the PRB coal char within the MWDRM reaction. $\mathrm{Fe}_{3} \mathrm{O}_{4}$ and $\mathrm{SrMoO}_{4}$ did not provide any benefit to the PRB coal char either mixed or precipitated on the surface. Like the bare $\mathrm{Fe}_{3} \mathrm{O}_{4}$ particles themselves, morphology again was a determining factor in reforming gas conversion rates, however, how it directly affected them 
in the presence of coal char was unclear. Of the shaped morphologies that were mixed with char, the micron-sized platelet particles had the highest of the reforming rates, although this was still lower than that of the stock $\mathrm{Fe}_{3} \mathrm{O}_{4}$ mixed with char. $\mathrm{SrMoO} /$ char experiments provided the greatest clarity for $\mathrm{Me}_{\mathrm{x}} \mathrm{O}_{\mathrm{y}} /$ char interactions. Higher weight ratios of $\mathrm{SrMoO}_{4}$ to char greatly reduced reforming capabilities. Through the reaction, $\mathrm{SrMoO}_{4}$ coarsens and covers nearby particles while char is eroded from the reaction with $\mathrm{CO}_{2}$. This combination was detrimental for the catalyst's reactionary capabilities, quickly stifling the reaction rate as basic active sites were both being covered and eroded. Additionally, the process in which $\mathrm{SrMoO}_{4}$ was incorporated with the char particles did not affect the reaction rate of the overall sample. Despite samples mixing methods, the conversion of the reforming gasses remained consistent. Temperature increases to samples of the same compositions nominally improved reforming capabilities but was limited by the amount of char removed from each example. Since char consistently degrades in the presence of $\mathrm{CO}_{2}$ at high temperatures, all catalysts beds lost considerable portions of char mass during each reaction.

$\mathrm{CaMoO}_{4}$ and $\mathrm{MoO}_{3}$ impregnated on PRB char showed the highest levels of reforming, included that of char itself. $\mathrm{CaMoO}_{4} /$ char decreased slowly as the reaction progressed while $\mathrm{MoO}_{3} /$ char ended testing with a reforming rate than where it had started. $\mathrm{CaMoO}_{4} / \mathrm{char}$ at $700{ }^{\circ} \mathrm{C}$ had the highest $\mathrm{CH}_{4}$ conversion average of $66.2 \%$ and had incredibly high stability reforming both gases. Also, this tested showed the high ethylene coupling capabilities, however the rate of formation was erratic. Although $\mathrm{CaMoO}_{4}$ and $\mathrm{MoO}_{3}$ did not possess the necessary dielectric properties to heat in the MW reaction environment, the char they were implanted on brought the materials to temperature. $\mathrm{CaMoO}_{4}$ and $\mathrm{MoO}_{3}$ seemed to reform $\mathrm{CH}_{4}$ and $\mathrm{CO}_{2}$ in unison with $\mathrm{PRB}$ char, as neither experienced drastic reductions in reforming gas conversion rates like that of the 
$\mathrm{SrMoO}_{4} /$ char and $\mathrm{Fe}_{3} \mathrm{O}_{4} /$ char samples. The dual reformation reaction of the inlet gasses allowed for stable, higher conversion rate averages than previous tests with other metal oxides. The higher conversion of the $\mathrm{Me}_{\mathrm{x}} \mathrm{O}_{\mathrm{y}} /$ char samples displayed clear support for possible use of char as a MW compatible substrate for DRM and like-reactions. Furthermore, continued research and design are strongly considered for combinations of catalytic metal oxide/PRB coal char as they have shown to improve the overall reactionary capabilities of certain reactions.

\subsection{Future Directions}

This study looks to continue applying a similar methodology of catalytic particle development with new metal oxide particles and PRB coal char. Dried PRB coal char is an interesting choice as a methane cracking catalyst with viable utility within hydrocarbon reactionary processes. New catalytic materials that lack necessary basic sites for $\mathrm{CO}_{2}$ adsorption should consider using PRB char as a viable substrate. Alternative particle packing methods of catalytic particles and char, as well as adjustments in the $\mathrm{CH}_{4} / \mathrm{CO}_{2}$ feed ratio would provide better insight to the catalytic interactions between the feed gasses and individual particles. PRB coal char may be of great consideration for other hydrocarbon reactions to improve reactionary yields when using microwave radiation. Ultimately, the use of microwave radiation as a heating source for chemical reactions requires more research but demonstrates its superiority to conventional heating methods when combined with the correct materials or solvents.

$\mathrm{CO}_{2}$ 


\section{References}

[1] “1.1. Coal \& Biomass - Coal Ranks.” Netl.doe.gov, www.netl.doe.gov/research/Coal/energysystems/gasification/gasifipedia/coal.

[2] Abdelsayed, Victor, et al. "MW-Assisted Conversion of Low Rank Coal under Methane Environment.” Energy \& Fuels, vol. 33, no. 2, Sept. 2019, pp. 905-915., doi:10.1021/acs.energyfuels.8b03805.

[3] Aika, K., \& Nishiyama, T. (1989). Utilization of CO2 in the OXIDATIVE coupling reaction of methane OVER CaO based catalysts. Catalysis Today, 4(3-4), 271-278.

doi:10.1016/0920-5861(89)85023-0

[4] Anthony, D., Howard, J., Hottel, H., \& Meissner, H. (1976). Rapid devolatilization and hydrogasification of bituminous coal. Fuel, 55(2), 121-128. doi:10.1016/00162361(76)90008-9

[5] Aramouni, N. A., Touma, J. G., Tarboush, B. A., Zeaiter, J., \& Ahmad, M. N. (2018). Catalyst design for dry reforming of methane: Analysis review. Renewable and Sustainable Energy Reviews, 82, 2570-2585. https://doi.org/10.1016/j.rser.2017.09.076

[6] Aziz, M. A., Jalil, A. A., Wongsakulphasatch, S., Vo, D. N. (2020). Understanding the role of surface basic sites of catalysts in co2 activation in dry reforming of methane: A short review. Catalysis Science Technology, 10(1), 35-45. doi:10.1039/c9cy01519a

[7] Bai, Xinwei, et al. "MW Catalytic Reactor for Upgrading Stranded Shale Gas to Aromatics." Fuel, vol. 243, 27 Jan. 2019, pp. 485-492., doi:10.1016/j.fuel.2019.01.147.

[8] Barbosa, L., Ardila, D., Cusatis, C., \& Andreeta, J. (2002). Growth and characterization of crack-free scheelite calcium molybdate single crystal fiber. Journal of Crystal Growth, 235(1-4), 327-332. doi:10.1016/s0022-0248(01)01816-4

[9] Bartholomew, Calvin H (2001). "Mechanisms of catalyst deactivation". Applied Catalysis A: General. 212 (1-2): 17-60. doi:10.1016/S0926-860X(00)00843-7.

[10] BERMUDEZ, J. M., FIDALGO, B., ARENILLAS, A., \& MENENDEZ, J. A. (2012). Mixtures of steel-making slag and carbons as catalyst for MW-assisted dry reforming of ch4. Chinese Journal of Catalysis, 33(7-8), 1115-1118. doi:10.1016/s1872-2067(11)60386-0

[11] Bian, Z., Das, S., Wai, M. H., Hongmanorom, P., \& Kawi, S. (2017). A review On Bimetallic nickel-based catalysts for co2 reforming of methane. ChemPhysChem, 18(22), 3117-3134. doi:10.1002/cphc.201700529

[12] Bitter, J., Seshan, K., \& Lercher, J. (1998). Mono and Bifunctional pathways Of Co2/ch4reforming over pt and RH based catalysts. Journal of Catalysis, 176(1), 93-101. doi:10.1006/jcat.1998.2022

[13] Bouchoul, N., Fourré, E., Tatibouët, J., \& Batiot-Dupeyrat, C. (2019). Plasma-Catalytic dry reforming of $\mathrm{CH} 4$ over Calcium OXIDE: CATALYST structural and TEXTURAL MODIFICATIONS. Plasma Chemistry and Plasma Processing, 39(3), 713-727. doi:10.1007/s11090-019-09966-9

[14] Boudart, M. "Turnover Rates in Heterogeneous Catalysis." American Chemical Society, vol. 95, no. 3, 1995, pp. 661-666., doi:10.1021/cr00035a009.

[15] Buevskaya, O. V., Suleimanov, A. I., Aliev, S. M., \& Sokolovskii, V. D. (1987). Activation of hydrocarbons in the oxidative dimerization of methane over alkaline earth metals. Reaction Kinetics and Catalysis Letters, 33(1), 223-227. doi:10.1007/bf02066728 
[16] Burda, Clemens, et al. "Chemistry and Properties of Nanocrystals of Different Shapes." ChemInform, vol. 36, no. 27, 18 Mar. 2005, pp. 1027-1087., doi:10.1002/chin.200527215.

[17] Cai, X., Hu, Y. H. (2019). Advances in catalytic conversion of methane and carbon dioxide to highly valuable products. Energy Science Engineering, 7(1), 4-29. doi:10.1002/ese3.278

[18] Cadigan, C. A., Corpuz, A. R., Lin, F., Caskey, C. M., Finch, K. B., Wang, X., \& Richards, R. M. (2013). ChemInform abstract: NANOSCALE (111) FACETED Rock-Salt metal oxides in catalysis. ChemInform, 44(22). doi:10.1002/chin.201322201

[19] Çiftyürek, E., Sabolsky, K., \& Sabolsky, E. M. (2017). High temperature SELECTIVE sensing of hydrogen with mgo-modified SRMOO4 micro-fibers. Sensors and Actuators B: Chemical, 249, 296-310. doi:10.1016/j.snb.2017.04.034

[20] Claridge, J. B., York, A. P., Brungs, A. J., Marquez-Alvarez, C., Sloan, J., Tsang, S. C., Green, M. L. (1998). New catalysts for the conversion of methane to synthesis gas:

Molybdenum and tungsten carbide. Journal of Catalysis, 180(1), 85-100. doi:10.1006/jcat.1998.2260

[21] Cong, L., Zhao, Y., Li, S., \& Sun, Y. (2017). Sr-doping effects on la 2 o 3 catalyst for oxidative coupling of methane. Chinese Journal of Catalysis, 38(5), 899-907. doi:10.1016/s1872-2067(17)62823-7

[22] Cordente, N., Respaud, M., Senocq, F., Casanove, M., Amiens, C., \& Chaudret, B. (2001). Synthesis and magnetic properties of nickel nanorods. Nano Letters, 1(10), 565-568. doi:10.1021/nl0100522

[23] Cushing, Brian L., et al. "Recent Advances in the Liquid-Phase Syntheses of Inorganic Nanoparticles." ChemInform, vol. 35, no. 47, 20 Aug. 2004, pp. 3894-3940., doi:10.1002/chin.200447224.

[24] De Dios García, I., Stankiewicz, A., Nigar, H. (2021). Syngas production via MWassisted dry reforming of methane. Catalysis Today, 362, 72-80. doi:10.1016/j.cattod.2020.04.045

[25] Dias, J., \& Assaf, J. (2003). Influence of calcium content in ni/cao/ $\gamma$-al2o3 catalysts FOR CO2-REFORMING of methane. Catalysis Today, 85(1), 59-68. doi:10.1016/s09205861(03)00194-9

[26] Ding, Y., Liu, F., Jiang, Q., Du, B., \& Sun, H. (2012). 12-Hydrothermal synthesis and characterization of fe3o4 nanorods. Journal of Inorganic and Organometallic Polymers and Materials, 23(2), 379-384. doi:10.1007/s10904-012-9789-2

[27] Djinović, P., Črnivec, I. G., Batista, J., Levec, J., Pintar, A. (2011). Catalytic syngas production from greenhouse gasses: Performance comparison Of RU-AL2O3 AND RHCEO2 CATALYSTS. Chemical Engineering and Processing: Process Intensification, 50(10), 1054-1062. doi:10.1016/j.cep.2011.05.018

[28] Dlouhý, T. "Low-Rank Coal Properties, Upgrading and Utilization for Improving Fuel Flexibility of Advanced Power Plants." Advanced Power Plant Materials, Design and Technology, 2010, pp. 291-311., doi:10.1533/9781845699468.3.291.

[29] Egiebor, Nosa O., and Murray R. Gray. "Evidence for Methane Reactivity during Coal Pyrolysis and Liquefaction.” Fuel, vol. 69, no. 10, 24 Apr. 1990, pp. 1276-1282., doi:10.1016/0016-2361(90)90289-3.

[30] Elert, G. (n.d.). Dielectrics. Retrieved June 27, 2021, from https://physics.info/dielectrics/ 
[31] Erdőhelyi, A., Fodor, K., Németh, R., Hancz, A., \& Oszkó, A. (2001). Partial oxidation of methane on silica-supported different alkali metal molybdates. Journal of Catalysis, 199(2), 328-337. doi:10.1006/jcat.2001.3178

[32] Ereña, J. (2020). Catalysts for syngas production. Catalysts, 10(6), 657. https://doi.org/10.3390/catal10060657

[33] Eryildirim, B., Arbag, H., Oktar, N., \& Dogu, G. (2021). Comparison of microwave and conventionally heated reactor performances in catalytic dehydrogenation of ethane.

International Journal of Hydrogen Energy, 46(7), 5296-5310. doi:10.1016/j.ijhydene.2020.11.067

[34] Fan, M., Abdullah, A. Z., \& Bhatia, S. (2009). ChemInform abstract: Catalytic technology for carbon Dioxide reforming of methane to synthesis gas. ChemInform, 40(50). doi:10.1002/chin.200950270

[35] Feng, L., Jiang, L., Mai, Z., \& Zhu, D. (2004). Polymer-controlled synthesis OF FE3O4 SINGLE-CRYSTAL NANORODS. Journal of Colloid and Interface Science, 278(2), 372375. doi:10.1016/j.jcis.2004.06.019

[36] Fidalgo, B., Arenillas, A., \& Menéndez, J. (2011). Mixtures of carbon and Ni/A12O3 as catalysts for The MW-assisted CO2 reforming of CH4. Fuel Processing Technology, 92(8), 1531-1536. doi:10.1016/j.fuproc.2011.03.015

[37] Fidalgo, B., Dominguez, A., Pis, J., \& Menendez, J. (2008). MW-assisted dry reforming of methane. International Journal of Hydrogen Energy, 33(16), 4337-4344. doi:1

[38] Frey, N. A., Peng, S., Cheng, K., \& Sun, S. (2009). ChemInform abstract: MAGNETIC Nanoparticles: Synthesis, functionalization, and applications in bioimaging and magnetic energy storage. ChemInform, 40(49). doi:10.1002/chin.200949268

[39] Frontera, P., Macario, A., Ferraro, M., \& Antonucci, P. (2017). Supported catalysts for co2 methanation: A review. Catalysts, 7(12), 59. doi:10.3390/catal7020059

[40] Gao, J., Liu, Q., Gu, F., Liu, B., Zhong, Z., \& Su, F. (2015). Recent advances in methanation catalysts for the production of synthetic natural gas. RSC Advances, 5(29), 22759-22776. doi:10.1039/c4ra16114a

[41] Gao, J., Wang, Y., Ping, Y., Hu, D., Xu, G., Gu, F., \& Su, F. (2012). A thermodynamic analysis of methanation reactions of carbon oxides for the production of synthetic natural gas. RSC Advances, 2(6), 2358. doi:10.1039/c2ra00632d

[42] Gao, J., Zheng, Y., Jehng, J., Tang, Y., Wachs, I. E., \& Podkolzin, S. G. (2015). Identification of molybdenum oxide nanostructures on zeolites for natural gas conversion. Science, 348(6235), 686-690. doi:10.1126/science.aaa7048

[43] Gao, X., Huang, C., Zhang, N., Li, J., Weng, W., \& Wan, H. (2008). Partial oxidation of methane to synthesis gas OVER Co/Ca/Al2O3 catalysts. Catalysis Today, 131(1-4), 211 218. doi:10.1016/j.cattod.2007.10.0510.1016/j.ijhydene.2008.05.056

[44] Guo, Jianjun, et al. "The Deposition of Coke from Methane on a Ni/MgAl2O4 Catalyst." Carbon, vol. 45, no. 6, 2007, pp. 1314-1321., doi:10.1016/j.carbon.2007.01.011.

[45] Halder, Aditi, et al. "Symmetry and Shape Issues in Nanostructure Growth." Journal of Materials Chemistry, vol. 20, no. 23, 4 Mar. 2010, pp. 4763-4772., doi:10.1039/b925996a.

[46] Hamzehlouia, S., Jaffer, S. A., \& Chaouki, J. (2018). MW Heating-Assisted Catalytic Dry reforming of methane to SYNGAS. Scientific Reports, 8(1). doi:10.1038/s41598-01827381-6 
[47] Han, J., Wang, X., Yue, J., Gao, S., \& Xu, G. (2014). Catalytic upgrading of coal pyrolysis tar over char-based catalysts. Fuel Processing Technology, 122, 98-106. doi:10.1016/j.fuproc.2014.01.033

[48] Hao, Z., Zhu, Q., Jiang, Z., Hou, B., \& Li, H. (2009). Characterization of aerogel ni/al2o3 catalysts and investigation on their stability for ch4-co2 reforming in a fluidized bed. Fuel Processing Technology, 90(1), 113-121. doi:10.1016/j.fuproc.2008.08.004

[49] Hartman, Ted. "Characteristics of Coal." Fossil Consulting Services, Inc., 13 June 2019, www.fossilconsulting.com/2018/08/28/characteristics-of-coal/.

[50] He, T., Sun, Z., Wu, J., Xu, Z., Zhang, D., \& Han, D. (2014). Catalytic performance of coal char for the methane reforming process. Chemical Engineering \& Technology, 38(1), 68-74. doi:10.1002/ceat.201400124

[51] Hosseinpour-Mashkani, S. S., Hosseinpour-Mashkani, S. S., \& Sobhani-Nasab, A. (2016). Synthesis and characterization OF rod-like Camoo4 nanostructure via free surfactant sonochemical route and Its photocatalytic application. Journal of Materials Science: Materials in Electronics, 27(5), 4351-4355. doi:10.1007/s10854-016-4303-1

[52] HOU, Z., CHEN, P., FANG, H., ZHENG, X., YASHIMA, T. (2006). Production of synthesis gas via methane reforming with co2 on noble metals and small amount of noble(rh-) promoted ni catalysts. International Journal of Hydrogen Energy, 31(5), 555-561. doi:10.1016/j.ijhydene.2005.06.010

[53] Hu, J. Z., Kwak, J. H., Wang, Y., Peden, C. H., Zheng, H., Ma, D., \& Bao, X. (2009). Studies of the active sites for Methane Dehydroaromatization using Ultrahigh-field solidstate 95Mo NMR SPECTROSCOPY. The Journal of Physical Chemistry C, 113(7), 29362942. doi:10.1021/jp8107914

[54] Jing, Xuliang, et al. "Evaluation of CO2 Gasification Reactivity of Different Coal Rank Chars by Physicochemical Properties." Energy \& Fuels, vol. 27, no. 12, 13 Nov. 2013, pp. 7287-7293., doi:10.1021/ef401639v.

[55] Hu, C., Yang, H., Wong, N., Chen, Y., Gong, M., Tian, A., . . Li, W. (2003). Theoretical study on the mechanism of the reaction of ch4+ mgo. The Journal of Physical Chemistry A, 107(13), 2316-2323. doi:10.1021/jp021953h

[56] Hu, H., Wachs, I. E., \& Bare, S. R. (1995). Surface structures of SUPPORTED Molybdenum Oxide Catalysts: Characterization BY Raman and Mo L3-EDGE XANES. The Journal of Physical Chemistry, 99(27), 10897-10910. doi:10.1021/j100027a034

[57] Huang, J., \& Suib, S. L. (1993). Dimerization of methane through MW plasmas. The Journal of Physical Chemistry, 97(37), 9403-9407. doi:10.1021/j100139a025

[58] Huang, Z., Zhang, Y., \& Tang, F. (2005). Solution-phase synthesis of single-crystalline magnetic nanowires with high aspect ratio and uniformity. Chemical Communications, (3), 342. doi:10.1039/b410463c

[59] Jia, C., Sun, L., Luo, F., Han, X., Heyderman, L. J., Yan, Z., . . Raabe, J. (2008). LargeScale synthesis OF Single-crystalline iron Oxide Magnetic Nanorings. Journal of the American Chemical Society, 130(50), 16968-16977. doi:10.1021/ja805152t

[60] Julian, Ignacio, et al. "Non-Oxidative Methane Conversion in MW-Assisted Structured Reactors." Chemical Engineering Journal, vol. 377, 23 Aug. 2018, pp. 1-12., doi:10.1016/j.cej.2018.08.150.

[61] Karakaya, C., \& Kee, R. J. (2016). Progress in the direct catalytic conversion of methane to fuels and chemicals. Progress in Energy and Combustion Science, 55, 60-97. doi:10.1016/j.pecs.2016.04.003 
[62] Kraszewski, A. (Ed.). (1996). MW aquametry: electromagnetic wave interaction with water-containing materials. IEEE.

[63] Labinger, Jay A. "Oxidative Coupling of Methane: An Inherent Limit to Selectivity?" Catalysis Letters, vol. 1, no. 11, 1988, pp. 371-375., doi:10.1007/bf00766166.

[64] Lam, S. S., Liew, R. K., Cheng, C. K., \& Chase, H. A. (2015). Catalytic MW pyrolysis of waste engine oil using metallic pyrolysis char. Applied Catalysis B: Environmental, 176177, 601-617. doi:10.1016/j.apcatb.2015.04.014

[65] Laursen, Anders B.; Man, Isabela Costinela; Trinhammer, Ole L.; Rossmeisl, Jan; Dahl, Søren (2011-10-04). "The Sabatier Principle Illustrated by Catalytic H2O2 Decomposition on Metal Surfaces". Journal of Chemical Education. 88 (12): 1711-

1715. doi:10.1021/ed101010x.

[66] Lee, J. "Molybdenum Carbide Catalysts II. Topotactic Synthesis of Unsupported Powders." Journal of Catalysis, vol. 112, no. 1, 1988, pp. 44-53., doi:10.1016/00219517(88)90119-4.

[67] Lezcano-González, I., Oord, R., Rovezzi, M., Glatzel, P., Botchway, S. W., Weckhuysen, B. M., \& Beale, A. M. (2016). Rücktitelbild: Molybdenum speciation and its impact on catalytic activity during Methane Dehydroaromatization IN Zeolite ZSM-5 as revealed By Operando X-Ray Methods (Angew. CHEM. 17/2016). Angewandte Chemie, 128(17), 54345434. doi:10.1002/ange.201602430

[68] Li, H., Zhang, C., Pang, C., Li, X., \& Gao, X. (2020). The advances in the special MW effects of the heterogeneous catalytic reactions. Frontiers in Chemistry, 8. doi:10.3389/fchem.2020.00355

[69] Li, L., Chen, J., Yan, K., Qin, X., Feng, T., Wang, J., . . Song, Z. (2018). Methane dry reforming with MW heating over carbon-based catalyst obtained by agriculture residues pyrolysis. Journal of CO2 Utilization, 28, 41-49. doi:10.1016/j.jcou.2018.09.010

[70] Li, L., Jiang, X., Wang, H., Wang, J., Song, Z., Zhao, X., \& Ma, C. (2017). Methane dry and mixed reforming on the mixture of bio-char and nickel-based catalyst with MW assistance. Journal of Analytical and Applied Pyrolysis, 125, 318-327. doi:10.1016/j.jaap.2017.03.009

[71] Li, L., Yan, K., Chen, J., Feng, T., Wang, F., Wang, J., . . Ma, C. (2019). Fe-rich biomass derived char for MW-assisted methane reforming with carbon dioxide. Science of The Total Environment, 657, 1357-1367. doi:10.1016/j.scitotenv.2018.12.097

[72] Li, M. Y., \& Sui, X. D. (2012). Synthesis and characterization of Magnetite particles by co-precipitation method. Key Engineering Materials, 512-515, 82-85.

doi:10.4028/www.scientific.net/kem.512-515.82

[73] Li, N., Huang, G., Li, Y., Xiao, H., Feng, Q., Hu, N., \& Fu, S. (2017). Enhanced MW absorption performance of coated carbon nanotubes by optimizing the fe3o4 nanocoating structure. ACS Applied Materials \& Interfaces, 9(3), 2973-2983. doi:10.1021/acsami.6b13142

[74] Li, X., Qu, X., Xu, Z., Dong, W., Wang, F., Guo, W., . . Du, Y. (2019). Fabrication of three-dimensional flower-like heterogeneous fe3o4/fe particles with tunable chemical composition and MW absorption performance. ACS Applied Materials \& Interfaces, 11(21), 19267-19276. doi:10.1021/acsami.9b01783

[75] Li, Yong, et al. "Morphology-Dependent Nanocatalysis: Metal Particles." Dalton Transactions, vol. 40, no. 22, 1 Feb. 2011, pp. 5811-5826., doi:10.1039/c0dt01404d. 
[76] Lian, S., Kang, Z., Wang, E., Jiang, M., Hu, C., \& Xu, L. (2003). Convenient synthesis of single crystalline magnetic fe3o4 nanorods. Solid State Communications, 127(9-10), 605608. doi:10.1016/s0038-1098(03)00580-5

[77] Lisjak, D., \& Mertelj, A. (2018). Anisotropic magnetic nanoparticles: A review of their properties, syntheses and potential applications. Progress in Materials Science, 95, 286-328. doi:10.1016/j.pmatsci.2018.03.003

[78] Liu, D., Cheo, W. N., Lim, Y. W., Borgna, A., Lau, R., \& Yang, Y. (2010). A comparative study on catalyst deactivation of nickel and cobalt incorporated mcm-41 catalysts modified by platinum in methane reforming with carbon dioxide. Catalysis Today, 154(3-4), 229-236. doi:10.1016/j.cattod.2010.03.054

[79] Liu, J., Hu, H., Jin, L., Wang, P., Zhu, S. (2010). Integrated coal PYROLYSIS with CO2 reforming of methane OVER Ni/MgO catalyst for IMPROVING Tar yield. Fuel Processing Technology, 91(4), 419-423. doi:10.1016/j.fuproc.2009.05.003

[80] Liu, J., Liu, G., Yuan, C., Chen, L., Tian, X., \& Fang, M. (2018). Fe3O4/ZnFe2O4 Micro/nanostructures and their HETEROGENEOUS EFFICIENT FENTON-LIKE visiblelight Photocatalysis process. New Journal of Chemistry, 42(5), 3736-3747. doi:10.1039/c7nj04548d

[81] Liu, S., Wang, L., Ohnishi, R., \& Ichikawa, M. (1999). Bifunctional catalysis of Mo/hzsm-5 in The Dehydroaromatization of methane to benzene And naphthalene XAFS/TG/DTA/MASS/FTIR characterization and supporting effects. Journal of Catalysis, 181(2), 175-188. doi:10.1006/jcat.1998.2310

[82] Longo, V. M., Cavalcante, L. S., Paris, E. C., Sczancoski, J. C., Pizani, P. S., Li, M. S., . . . Varela, J. A. (2011). Hierarchical assembly of CaMoO4 Nano-Octahedrons and THEIR Photoluminescence properties. The Journal of Physical Chemistry C, 115(13), 5207-5219. doi:10.1021/jp1082328

[83] Luo, Y., Dai, X., Zhang, W., Yang, Y., Sun, C. Q., \& Fu, S. (2010). Controllable synthesis and luminescent properties of novel erythrocyte-like camoo4hierarchical nanostructuresvia a simple surfactant-free hydrothermal route. Dalton Trans., 39(9), 22262231. doi:10.1039/b915099d

[84] Maciel, L. J., Souza, A. E., Vasconcelos, S. M., Knoechelmann, A., \& Augusto Moraes de Abreu, C. (2007). Dry reforming and partial oxidation of natural gas to syngas production. Studies in Surface Science and Catalysis, 469-474. doi:10.1016/s01672991(07)80176-5

[85] Maiti, G. (1998). Dehydrogenative coupling Of CH4: Nature of interaction OVER CaO surface. Recent Advances In Basic and Applied Aspects of Industrial Catalysis, Proceedings of 13th National Symposium and Silver Jubilee Symposium of Catalysis of India, 487-495. doi:10.1016/s0167-2991(98)80323-6

[86] Majhi, S., Mohanty, P., Wang, H., \& Pant, K. (2013). Direct conversion of natural gas to higher hydrocarbons: A review. Journal of Energy Chemistry, 22(4), 543-554.

doi:10.1016/s2095-4956(13)60071-6

[87] Mamonov, N. A., Fadeeva, E. V., Grigoriev, D. A., Mikhailov, M. N., Kustov, L. M., \& Alkhimov, S. A. (2013). Metal/zeolite catalysts of methane dehydroaromatization. Russian Chemical Reviews, 82(6), 567-585. doi:10.1070/rc2013v082n06abeh004346

[88] Marques, V. S., Cavalcante, L. S., Sczancoski, J. C., Alcântara, A. F., Orlandi, M. O., Moraes, E., ... Santos, M. R. (2010). Effect of different solvent ratios (water/ethylene 
glycol) on the growth process of camoo4crystals and their optical properties. Crystal Growth \& Design, 10(11), 4752-4768. doi:10.1021/cg100584b

[89] Meng, F., Yu, J., Tahmasebi, A., Han, Y., Zhao, H., Lucas, J., \& Wall, T. (2013). Characteristics of chars from low-temperature pyrolysis of lignite. Energy \& Fuels, 28(1), 275-284. doi:10.1021/ef401423s

[90] Metaxas, A. C., \& Meredith, R. J. (1988). Industrial MW heating. doi:10.1049/pbpo004e

[91] Mevawala, C., Bai, X., Bhattacharyya, D., \& Hu, J. (2021). Dynamic data reconciliation, parameter estimation, and multi-scale, multi-physics modeling of the microwave-assisted methane dehydroaromatization process. Chemical Engineering Science, 239, 116624. https://doi.org/10.1016/j.ces.2021.116624

[92] Michorczyk, P., Węgrzyniak, A., Węgrzynowicz, A., \& Handzlik, J. (2019). Simple and efficient way of molybdenum oxide-based catalyst activation for olefins metathesis by methane pretreatment. ACS Catalysis, 9(12), 11461-11467. doi:10.1021/acscatal.9b03714

[93] Minakshi, M., Mitchell, D. R., Baur, C., Chable, J., Barlow, A. J., Fichtner, M., . . . Ahuja, R. (2019). Phase evolution in calcium molybdate nanoparticles as a function of synthesis temperature and its electrochemical effect on energy storage. Nanoscale Advances, 1(2), 565-580. doi:10.1039/c8na00156a

[94] Monsef-Mirzai, Parisa, et al. "Rapid MW Pyrolysis of Coal." Fuel and Energy Abstracts, vol. 36, no. 4, 1995, pp. 20-27., doi:10.1016/0140-6701(95)95547-i.

[95] Ni, S., Sun, X., Wang, X., Zhou, G., Yang, F., Wang, J., \& He, D. (2010). Low temperature synthesis of fe3o4 micro-spheres and its MW absorption properties. Materials Chemistry and Physics, 124(1), 353-358. doi:10.1016/j.matchemphys.2010.06.046

[96] Nikoo, M. K., Amin, N. (2011). Thermodynamic analysis of carbon dioxide reforming of methane in view of solid carbon formation. Fuel Processing Technology, 92(3), 678-691. doi:10.1016/j.fuproc.2010.11.027

[97] Olivos-Suarez, A. I., Szécsényi, À, Hensen, E. J., Ruiz-Martinez, J., Pidko, E. A., \& Gascon, J. (2016). Strategies for the direct catalytic valorization of methane using heterogeneous catalysis: Challenges and opportunities. ACS Catalysis, 6(5), 2965-2981. doi:10.1021/acscatal.6b00428

[98] Ovalles, Cesar, et al. "04/00109 Use of a Dispersed Iron Catalyst for Upgrading ExtraHeavy Crude Oil Using Methane as Source of Hydrogen." Fuel and Energy Abstracts, vol. 45, no. 1, 2004, pp. 887-892., doi:10.1016/s0140-6701(04)91311-2.

[99] Ozer, Mustafa, et al. "Effect of Coal Nature on the Gasification Process." Integrated Gasification Combined Cycle (IGCC) Technologies, 2017, pp. 257-304. Web of Science, doi:10.1016/b978-0-08-100167-7.00007-x.

[100] Pakhare, D., Spivey, J. (2014). A review of dry (co2) reforming of methane over noble metal catalysts. Chem. Soc. Rev., 43(22), 7813-7837. doi:10.1039/c3cs60395d

[101] Palma, V., Barba, D., Cortese, M., Martino, M., Renda, S., \& Meloni, E. (2020). MWs and Heterogeneous CATALYSIS: A review on Selected Catalytic processes. Catalysts, 10(2), 246. doi:10.3390/catal10020246

[102] Pang, L. (1997). Reactivity of methane at low temperatures $\left(\sim 400^{\circ} \mathrm{c}\right)$ with 'refractory' bonds. Fuel, 76(12), 1091-1104. doi:10.1016/s0016-2361(97)00105-1

[103] Patil, P., Gude, V. G., Pinappu, S., \& Deng, S. (2011). Transesterification kinetics of CAMELINA sativa oil on metal oxide catalysts under conventional and MW heating conditions. Chemical Engineering Journal, 168(3), 1296-1300. doi:10.1016/j.cej.2011.02.030 
[104] Peng, Zhiwei, et al. "Dielectric Characterization of Indonesian Low-Rank Coal for MW Processing." Fuel Processing Technology, vol. 156, 2017, pp. 171-177., doi:10.1016/j.fuproc.2016.11.001.

[105] Petcharoen, K., \& Sirivat, A. (2012). Synthesis and characterization of magnetite nanoparticles via the chemical co-precipitation method. Materials Science and Engineering: B, 177(5), 421-427. doi:10.1016/j.mseb.2012.01.003

[106] Petricci, E., Cini, E., \& Taddei, M. (2020). Metal catalysis with MWs in organic synthesis: A personal account. European Journal of Organic Chemistry, 2020(29), 44354446. doi:10.1002/ejoc.202000092

[107] Priecel, P., \& Lopez-Sanchez, J. A. (2018). Advantages and limitations of Microwave REACTORS: From chemical synthesis to the Catalytic valorization Of BIOBASED CHEMICALS. ACS Sustainable Chemistry \& Engineering, 7(1), 3-21. https://doi.org/10.1021/acssuschemeng.8b03286

[108] Prince, B. M., \& Cundari, T. R. (2013). Methane C-H Bond activation by "Naked" alkali METAL Imidyl and alkaline earth Metal Imide Complexes. the role of Ligand spin and Nucleophilicity. The Journal of Physical Chemistry A, 117(38), 9245-9251. doi:10.1021/jp404951e

[109] Raju, G. S., Pavitra, E., Ko, Y. H., \& Yu, J. S. (2012). A facile and efficient strategy for the preparation of Stable camoo4 SPHERULITES using Ammonium MOLYBDATE as a Molybdenum source and their Excitation Induced Tunable luminescent properties for OPTICAL applications. Journal of Materials Chemistry, 22(31), 15562. doi:10.1039/c2jm32049e

[110] Ranzi, E., et al. "Pyrolysis, Gasification, and Combustion of Solid Fuels." Thermochemical Process Engineering Advances in Chemical Engineering, 2016, pp. 1-94. Web of Science, doi:10.1016/bs.ache.2016.09.001.

[111] Rice, M. J., Chakraborty, A. K., \& Bell, A. T. (1999). Al next nearest Neighbor, Ring occupation, and PROXIMITY statistics In zsm-5. Journal of Catalysis, 186(1), 222-227. doi:10.1006/jcat.1999.2544

[112] Robinson, B., Caiola, A., Bai, X., Abdelsayed, V., Shekhawat, D., \& Hu, J. (2020). Catalytic direct conversion of ethane to value-added chemicals under microwave irradiation. Catalysis Today, 356, 3-10. https://doi.org/10.1016/j.cattod.2020.03.001

[113] Rodrigues, A. C., \& Monteiro, J. L. (2007). CO2 addition on the Non-oxidative Dehydroaromatization of methane OVER MOMCM-22. Catalysis Letters, 117(3-4), 166-170. doi:10.1007/s10562-007-9130-9

[114] Santos, A. L., Reis, R. A., Rossa, V., Reis, M. M., Costa, A. L., Veloso, C. O., . . . Chiaro, S. S. (2012). Silica-alumina impregnated WITH cerium, nickel, and Molybdenum oxides for adsorption of sulfur and nitrogen compounds from diesel. Materials Letters, 83, 158-160. doi:10.1016/j.matlet.2012.06.011

[115] Sathe, Chirag, et al. "Release of Volatiles from the Pyrolysis of a Victorian Lignite at Elevated Pressures.” Fuel, vol. 81, no. 9, 26 Feb. 2002, pp. 1171-1178., doi:10.1016/s00162361(02)00033-9.

[116] Scaccia, S., Calabrò, A., \& Mecozzi, R. (2012). Investigation of the evolved gases from Sulcis coal DURING PYROLYSIS Under N2 and h2 atmospheres. Journal of Analytical and Applied Pyrolysis, 98, 45-50. doi:10.1016/j.jaap.2012.05.001

[117] Schobert, H. (2017). Introduction to low-rank coals. Low-Rank Coals for Power Generation, Fuel and Chemical Production, 3-21. doi:10.1016/b978-0-08-100895-9.00001-2 
[118] Schrittwieser, S., Reichinger, D., \& Schotter, J. (2017). Applications, surface modification and functionalization of nickel nanorods. Materials, 11(1), 45. doi:10.3390/ma11010045

[119] Schubert, H., \& Regier, M. (2005). The MW processing of foods. Woodhead.

[120] Schwach, P., Hamilton, N., Eichelbaum, M., Thum, L., Lunkenbein, T., Schlögl, R., \& Trunschke, A. (2015). Structure sensitivity of the oxidative activation of methane OVER $\mathrm{MgO}$ Model catalysts: Ii. nature of active sites and reaction mechanism. Journal of Catalysis, 329, 574-587. doi:10.1016/j.jcat.2015.05.008

[121] Schwach, P., Pan, X., Bao, X. (2017). Direct conversion of methane to value-added chemicals over heterogeneous catalysts: Challenges and prospects. Chemical Reviews, 117(13), 8497-8520. doi:10.1021/acs.chemrev.6b00715

[122] Sharifvaghefi, S., Shirani, B., Eic, M., \& Zheng, Y. (2019). Application of MW in hydrogen production from Methane Dry Reforming: Comparison between the conventional AND MW-Assisted Catalytic Reforming on improving the energy efficiency. Catalysts, 9(7), 618. doi:10.3390/cata19070618

[123] Singha, R. K., Shukla, A., Sandupatla, A., Deo, G., Bal, R. (2017). Synthesis and catalytic activity of a Pd doped NI-MGO catalyst for Dry reforming of methane. Journal of Materials Chemistry A, 5(30), 15688-15699. doi:10.1039/c7ta04452f

[124] Singh, S., et al. "MW Assisted Coal Conversion." Fuel, vol. 140, 2015, pp. 495-501., doi:10.1016/j.fuel.2014.09.108.

[125] Solomon, Peter R., et al. "Coal Pyrolysis: Experiments, Kinetic Rates and Mechanisms." Progress in Energy and Combustion Science, vol. 18, no. 2, 1992, pp. 133 220., doi:10.1016/0360-1285(92)90021-r.

[126] Solvent choice for MW synthesis. (n.d.). Retrieved June 28, 2021, from https://cem.com/en/MW-chemistry/solvent-choice

[127] Solymosi, F., Cserényi, J., Szöke, A., Bánsági, T., \& Oszkó, A. (1997). Aromatization of Methane OVER supported and Unsupported MO-BASED CATALYSTS. Journal of Catalysis, 165(2), 150-161. doi:10.1006/jcat.1997.1478

[128] Solymosi, F., Erd?helyi, A., \& Sz?ke, A. (1995). Dehydrogenation of methane on supported molybdenum oxides. formation of BENZENE from methane. Catalysis Letters, 32(1-2), 43-53. doi:10.1007/bf00806100

[129] Solymosi, F., Kuts?n, G., \& Erd?helyi, A. (1991). Catalytic reaction of CH4 with CO2 OVER alumina-supported PT metals. Catalysis Letters, 11(2), 149-156.

doi:10.1007/bf00764080

[130] Solymosi, F., Sz $>$ ke, A., \& Cser nyi, J. (1996). Conversion of methane to benzene over Mo2c AND Mo2C/ZSM-5 catalysts. Catalysis Letters, 39(3-4), 157-161. doi:10.1007/bf00805576

[131] Sun, S., \& Zeng, H. (2002). Size-controlled synthesis of magnetite nanoparticles. Journal of the American Chemical Society, 124(28), 8204-8205. doi:10.1021/ja026501x

[132] Sun, Z., Wu, J., \& Zhang, D. (2008). Co2and h2o gasification kinetics of a coal char in the presence of methane. Energy \& Fuels, 22(4), 2160-2165. doi:10.1021/ef8000949

[133] Sun, Zhi-Qiang, et al. "Methane Cracking over a Bituminous Coal Char.” Energy \& Fuels, vol. 21, no. 3, 2007, pp. 1601-1605., doi:10.1021/ef060616v.

[134] Szöke, A., \& Solymosi, F. (1996). Selective oxidation of methane to benzene over k2moo4/zsm-5 catalysts. Applied Catalysis A: General, 142(2), 361-374. doi:10.1016/0926$860 x(96) 00085-3$ 
[135] Taghizadeh, F. (2016). The study of structural and magnetic properties OF Nio

Nanoparticles. Optics and Photonics Journal, 06(08), 164-169. doi:10.4236/opj.2016.68b027

[136] Tahmasebi, Arash, et al. "Experimental Study on MW Drying of Chinese and Indonesian Low-Rank Coals." Fuel Processing Technology, vol. 92, no. 10, 30 June 2011, pp. 18211829., doi:10.1016/j.fuproc.2011.04.004.

[137] Taifan, W., \& Baltrusaitis, J. (2016). CH 4 conversion to value added products: Potential, limitations and extensions of a single step heterogeneous catalysis. Applied Catalysis B:

Environmental, 198, 525-547. doi:10.1016/j.apcatb.2016.05.081

[138] Takanabe, K. (2012). Catalytic conversion of methane: Carbon dioxide reforming and oxidative coupling. Journal of the Japan Petroleum Institute, 55(1), 1-12.

doi:10.1627/jpi.55.1

[139] Teshima, K., Yubuta, K., Sugiura, S., Fujita, Y., Suzuki, T., Endo, M., . . Oishi, S. (2006). Selective growth of Calcium molybdate Whiskers by rapid cooling of a sodium Chloride Flux. Crystal Growth \& Design, 6(7), 1598-1601. doi:10.1021/cg050673z

[140] Thybaut, Joris W., et al. "A Novel Technology for Natural Gas Conversion by Means of Integrated Oxidative Coupling and Dry Reforming of Methane.” Chemie Ingenieur Technik, vol. 86, no. 11, 2014, pp. 1855-1870., doi:10.1002/cite.201400068.

[141] Tsodikov, M. V., Ellert, O. G., Nikolaev, S. A., Arapova, O. V., Bukhtenko, O. V., Maksimov, Y. V., . . Vasil'kov, A. Y. (2018). Fe-containing nanoparticles used as effective catalysts of lignin reforming to syngas and hydrogen assisted by MW irradiation. Journal of Nanoparticle Research, 20(3). doi:10.1007/s11051-018-4185-7

[142] Tsyganok, A. I., Inaba, M., Tsunoda, T., Hamakawa, S., Suzuki, K., Hayakawa, T. (2003). Dry reforming of methane over supported noble metals: A novel approach to preparing catalysts. Catalysis Communications, 4(9), 493-498. doi:10.1016/s15667367(03)00130-4

[143] "U.S. Energy Information Administration - EIA - Independent Statistics and Analysis." How Much Coal Is Left - U.S. Energy Information Administration (EIA), 12 Nov. 2019, www.eia.gov/energyexplained/coal/how-much-coal-is-left.php.

[144] "U.S. Energy Information Administration - EIA - Independent Statistics and Analysis." Use of Coal - U.S. Energy Information Administration (EIA), U.S. Energy Information Administration, 9 May 2019, www.eia.gov/energyexplained/coal/use-ofcoal.php.

[145] Usman, M., Wan Daud, W., Abbas, H. F. (2015). Dry reforming of methane: Influence of process parameters - a review. Renewable and Sustainable Energy Reviews, 45, 710-744. doi:10.1016/j.rser.2015.02.026

[146] Wan, J., Chen, X., Wang, Z., Yang, X., \& Qian, Y. (2005). A soft-template-assisted hydrothermal approach to single-crystal fe3o4 nanorods. Journal of Crystal Growth, 276(34), 571-576. doi:10.1016/j.jcrysgro.2004.11.423

[147] Wan, J. K. S., et al. "Highly Effective Methane Conversion to Aromatic Hydrocarbons by Means of MW and Rf-Induced Catalysis." Research on Chemical Intermediates, vol. 26, no. 6, 18 May 2000, pp. 599-619., doi:10.1163/156856700x00561.

[148] Wang, D., Lunsford, J. H., \& Rosynek, M. P. (1996). Catalytic conversion of methane to benzene over mo/zsm-5. Topics in Catalysis, 3(3-4), 289-297. doi:10.1007/bf02113855

[149] Wang, D., Lunsford, J. H., \& Rosynek, M. P. (1997). Characterization of A Mo/zsm-5 catalyst for the conversion of methane to benzene. Journal of Catalysis, 169(1), 347-358. doi:10.1006/jcat.1997.1712 
[150] Wang, J., Peng, Z., Huang, Y., \& Chen, Q. (2004). Growth of magnetite nanorods along Its Easy-magnetization axis of [110]. Journal of Crystal Growth, 263(1-4), 616-619. doi:10.1016/j.jcrysgro.2003.11.102

[151] Wang, M., Jin, L., Li, Y., Lv, J., Wei, B., \& Hu, H. (2018). In-situ catalytic upgrading of COAL pyrolysis TAR coupled with CO2 reforming of methane over ni-based catalysts. Fuel Processing Technology, 177, 119-128. doi:10.1016/j.fuproc.2018.04.022

[152] Wang, T, et al. "MW-Assisted Hydroconversions of Demineralized Coal Liquefaction Residues from Shenfu and Shengli Coals." Fuel, vol. 87, no. 4-5, 21 May 2007, pp. 498507., doi:10.1016/j.fuel.2007.04.016.

[153] Wang, Ting. "An Overview of IGCC Systems." Integrated Gasification Combined Cycle (IGCC) Technologies, 2017, pp. 1-80., doi:10.1016/b978-0-08-100167-7.00001-9.

[154] Wang, W., Hu, Y., Goebl, J., Lu, Z., Zhen, L., \& Yin, Y. (2009). Shape- and Sizecontrolled synthesis of Calcium molybdate DOUGHNUT-SHAPED MICROSTRUCTURES. The Journal of Physical Chemistry C, 113(37), 16414-16423. doi:10.1021/jp9059278

[155] Wang, X., Liu, Y., Han, H., Mølhave, K., \& Sun, H. (2017). Enhanced high-frequency MW absorption of fe3o4 architectures based on porous nanoflake. Ceramics International, 43(17), 16013-16017. doi:10.1016/j.ceramint.2017.08.111

[156] Wang, X., Wei, B., Huang, X., Fan, M., Wang, Y., \& Chen, X. (2020). Enhanced nearzero-CO2-emission chemicals-oriented oil production from coal with inherent $\mathrm{Co} 2$ RECYCLING: Part I-PRB coal FAST pyrolysis coupled With Co2/ch4 reforming. International Journal of Coal Science Technology, 7(3), 433-443. doi:10.1007/s40789-02000359-4

[157] Wei, L., Tan, Y., Han, Y., Zhao, J., Wu, J., \& Zhang, D. (2011). Hydrogen production by methane cracking over different coal chars. Fuel, 90(11), 3473-3479. doi:10.1016/j.fuel.2011.06.053

[158] Wei, Ling, et al. "Hydrogen Production by Methane Cracking over Xiaolongtan Lignite Chars: The Role of Mineral Matter.” Fuel, vol. 183, 27 June 2016, pp. 345-350., doi:10.1016/j.fuel.2016.06.095.

[159] Wei, Xianyong, et al. "Advances in the Study of Hydrogen Transfer to Model Compounds for Coal Liquefaction." Fuel Processing Technology, vol. 62, no. 2-3, 2000, pp. 103-107., doi:10.1016/s0378-3820(99)00114-9.

[160] Wiogo, H., Lim, M., Munroe, P., \& Amal, R. (2011). Understanding the formation of iron oxide nanoparticles with Acicular structure From iron(iii) chloride AND Hydrazine Monohydrate. Crystal Growth \& Design, 11(5), 1689-1696. doi:10.1021/cg101623n

[161] XING, S., ZHOU, D., CAO, L., \& LI, X. (2010). Density functional theory study on structure of MOLYBDENUM carbide and catalytic mechanism for METHANE activation over ZSM-5 Zeolite. Chinese Journal of Catalysis, 31(4), 415-422. doi:10.1016/s18722067(09)60062-0

[162] Xiong, G., Feng, Z., Li, J., Yang, Q., Ying, P., Xin, Q., \& Li, C. (2000). UV resonance Raman Spectroscopic studies on the genesis of highly Dispersed Surface Molybdate species on $\gamma$-Alumina. The Journal of Physical Chemistry B, 104(15), 3581-3588.

doi:10.1021/jp993609z

[163] $\mathrm{Xu}$, Longya, et al. "Combination of CH4 Oxidative Coupling Reaction with C2H6 Oxidative Dehydrogenation by CO2 to C2H4." Fuel, vol. 81, no. 11-12, 2002, pp. 15931597., doi:10.1016/s0016-2361(02)00074-1. 
[164] Xu, W. (2003). High pressure hydropyrolysis of coals by using a continuous free-fall reactor*. Fuel, 82(6), 677-685. doi:10.1016/s0016-2361(02)00340-x

[165] Xuan, S., Wang, Y. J., Yu, J. C., \& Cham-Fai Leung, K. (2009). Tuning the grain size and particle size of superparamagnetic fe3o4 microparticles. Chemistry of Materials, 21(21), 5079-5087. doi:10.1021/cm901618m

[166] Yan, A., Liu, Y., Liu, Y., Li, X., Lei, Z., \& Liu, P. (2012). A naac-assisted large-scale coprecipitation synthesis and MW absorption efficiency of fe3o4 nanowires. Materials Letters, 68, 402-405. doi:10.1016/j.matlet.2011.10.093

[167] Yan, Shuai, et al. "Catalytic Coal Hydrogasification by Cobalt-Calcium Catalyst in a Pressurized Fluidized Bed: Role of Hydropyrolysis and Catalysis Process.” Journal of Analytical and Applied Pyrolysis, vol. 135, 1 Sept. 2018, pp. 251-259., doi:10.1016/j.jaap.2018.08.028.

[168] Yang, H., Hu, C., \& Qin, S. (2006). Theoretical study on the reaction mechanism of CH4 with cao. Chemical Physics, 330(3), 343-348. doi:10.1016/j.chemphys.2006.07.046

[169] Yang, X. H., \& Tang, J. (2002). Advances in bioprocessing engineering. Advances in Agricultural Science \& Technology, 1-44. doi:10.1142/4763

[170] Yao, S., Yan, B., Jiang, Z., Liu, Z., Wu, Q., Lee, J. H., \& Chen, J. G. (2018). Combining $\mathrm{CO} 2$ reduction with Ethane Oxidative dehydrogenation by OXYGEN-MODIFICATION of MOLYBDENUM CARBIDE. ACS Catalysis, 8(6), 5374-5381. doi:10.1021/acscatal.8b00541

[171] Yin, Y., Gao, Y., Sun, Y., Zhou, B., Ma, L., Wu, X., \& Zhang, X. (2010). Synthesis and PHOTOLUMINESCENT properties of CAMOO4 Nanostructures at room temperature. Materials Letters, 64(5), 602-604. doi:10.1016/j.matlet.2009.12.014

[172] You, Hongjun. "Coke Deactivation Kinetics of the Aromatization Reaction." Petroleum Science and Technology, vol. 24, no. 7, 2006, pp. 829-838., doi:10.1081/lft-200041195.

[173] Yusoff, A. (n.d.). A review: Synthetic strategy control of magnetite nanoparticles production. Retrieved June 28, 2021, from https://doi.org/10.12989/anr.2018.6.1.001

[174] Zhan, Y., Zheng, C., Liu, Y., \& Wang, G. (2003). Synthesis of NIO nanowires by an Oxidation route. Materials Letters, 57(21), 3265-3268. doi:10.1016/s0167-577x(03)00045-4

[175] Zhang, J., Chen, J., \& Wang, Z. (2007). Surfactant-assisted preparation of singlecrystalline fe3o4 nanowires under low magnetic field. Materials Letters, 61(8-9), 1629-1632. doi:10.1016/j.matlet.2006.07.088

[176] Zhang, J., Jin, L., He, X., Liu, S., \& Hu, H. (2011). Catalytic methane decomposition Over activated Carbons prepared from direct coal liquefaction residue by $\mathrm{KOH}$ activation with addition of SIO 2 or Sba-15. International Journal of Hydrogen Energy, 36(15), 89788984. doi:10.1016/j.ijhydene.2011.04.205

[177] Zhang, Jun-Zhong, et al. "Molybdenum ZSM-5 Zeolite Catalysts for the Conversion of Methane to Benzene." Catalysis Today, vol. 44, no. 1-4, 1998, pp. 293-300., doi:10.1016/s0920-5861(98)00202-8.

[178] Zhang, F., Song, Z., Zhu, J., Liu, L., Sun, J., Zhao, X., . . Wang, W. (2018). Process of ch4-co2 reforming over Fe/SiC Catalyst Under MW irradiation. Science of The Total Environment, 639, 1148-1155. doi:10.1016/j.scitotenv.2018.04.364

[179] Zhang, F., Song, Z., Zhu, J., Sun, J., Zhao, X., Mao, Y., . . Wang, W. (2018). Factors influencing Ch4co2 Reforming reaction over FE catalyst supported on foam CERAMICS Under MW irradiation. International Journal of Hydrogen Energy, 43(20), 9495-9502. doi:10.1016/j.ijhydene.2018.03.171 
[180] Zhang, L., Li, L., Li, J., Zhang, Y., \& Hu, J. (2013). Carbon dioxide reforming of Methane over Nickel catalyst supported ON mgo(111) nanosheets. Topics in Catalysis, 57(69), 619-626. doi:10.1007/s11244-013-0220-1

[181] Zhang, L., Xu, S., Zhao, W., \& Liu, S. (2007). Co-pyrolysis of biomass and coal in a free fall reactor. Fuel, 86(3), 353-359. doi:10.1016/j.fuel.2006.07.004

[182] Zhang, T., Yang, J., Zhang, N., Huang, T., \& Wang, Y. (2017). Achieving large dielectric property improvement in poly(ethylene vinyl acetate)/thermoplastic polyurethane/multiwall carbon nanotube nanocomposites by tailoring phase morphology. Industrial \& Engineering Chemistry Research, 56(13), 3607-3617. doi:10.1021/acs.iecr.6b04763

[183] Zhang, W., Ma, D., Han, X., Liu, X., Bao, X., Guo, X., \& Wang, X. (1999). Methane Dehydro-aromatization OVER Mo/hzsm-5 in the absence of Oxygen: A Multinuclear Solidstate NMR study of the interaction between Supported mo species and HZSM-5 ZEOLITE with different Crystal Sizes. Journal of Catalysis, 188(2), 393-402. doi:10.1006/jcat.1999.2670

[184] Zhang, X., Dai, B., Zhu, A., Gong, W., \& Liu, C. (2002). The simultaneous activation of methane and carbon dioxide to $\mathrm{C} 2$ hydrocarbons UNDER Pulse corona plasma Over La2o3/ $\gamma$-al2o3 catalyst. Catalysis Today, 72(3-4), 223-227. doi:10.1016/s09205861(01)00496-5

[185] Zheng, H., Ma, D., Bao, X., Hu, J. Z., Kwak, J. H., Wang, Y., \& Peden, C. H. (2008). Direct observation of the active Center for Methane Dehydroaromatization using An ultrahigh Field95Mo NMR SPECTROSCOPY. Journal of the American Chemical Society, 130(12), 3722-3723. doi:10.1021/ja7110916

[186] Zhong, L., Hu, J., Liang, H., Cao, A., Song, W., \& Wan, L. (2006). Self-Assembled 3D FLOWERLIKE iron OXIDE Nanostructures and their application in water treatment. Advanced Materials, 18(18), 2426-2431. doi:10.1002/adma.200600504

[187] Zhou, D., Zuo, S., \& Xing, S. (2012). Methane dehydrogenation and coupling to Ethylene over A Mo/HZSM-5 Catalyst: A density functional theory study. The Journal of Physical Chemistry C, 116(6), 4060-4070. doi:10.1021/jp209037c

[188] Zhou, K., \& Li, Y. (2012). ChemInform abstract: Catalysis based on Nanocrystals with well-defined facets. ChemInform, 43(14). doi:10.1002/chin.201214213

[189] Zhu, P., Yu, Z., Zhang, J., Dai, B., Zhang, J., Liang, P., Lei, Z. (2017). Catalytic pyrolysis of bituminous coal under pyrolysis gas over a ni/mgo catalyst. Chemical Engineering Technology, 40(9), 1605-1610. doi:10.1002/ceat.201700163 